\title{
The Microvascular And Systemic Effects Of Titanium Dioxide Nanoparticle Exposure: A Physiological Perspective
}

\author{
Alaeddin Bashir Abukabda \\ ababukabda@mix.wvu.edu
}

Follow this and additional works at: https://researchrepository.wvu.edu/etd

Part of the Cellular and Molecular Physiology Commons, Immunity Commons, and the Nanomedicine Commons

\section{Recommended Citation}

Abukabda, Alaeddin Bashir, "The Microvascular And Systemic Effects Of Titanium Dioxide Nanoparticle Exposure: A Physiological Perspective" (2018). Graduate Theses, Dissertations, and Problem Reports. 3730.

https://researchrepository.wvu.edu/etd/3730

This Dissertation is protected by copyright and/or related rights. It has been brought to you by the The Research Repository @ WVU with permission from the rights-holder(s). You are free to use this Dissertation in any way that is permitted by the copyright and related rights legislation that applies to your use. For other uses you must obtain permission from the rights-holder(s) directly, unless additional rights are indicated by a Creative Commons license in the record and/ or on the work itself. This Dissertation has been accepted for inclusion in WVU Graduate Theses, Dissertations, and Problem Reports collection by an authorized administrator of The Research Repository @ WVU.

For more information, please contact researchrepository@mail.wvu.edu. 


\title{
THE MICROVASCULAR AND SYSTEMIC EFFECTS OF TITANIUM DIOXIDE NANOPARTICLE EXPOSURE: A PHYSIOLOGICAL PERSPECTIVE
}

\author{
ALAEDDIN BASHIR ABUKABDA \\ Dissertation submitted to the \\ School of Medicine at West Virginia University \\ In partial fulfillment of the requirements for the degree of
}

\author{
Doctor of Philosophy \\ in \\ Cellular and Integrative Physiology \\ Robert W. Brock, Ph.D., Chair \\ Timothy R. Nurkiewicz, Ph.D., Mentor \\ Christopher F. Cuff, Ph.D. \\ Aaron Erdely, Ph.D. \\ William T. Stauber, Ph.D. \\ Stephen J. Valentine, Ph.D. \\ Jesus A. Araujo, Ph.D., M.D., External Examiner \\ Department of Physiology and Pharmacology \\ Morgantown, West Virginia \\ 2018
}

Keywords: Microcirculation, Titanium dioxide, Nanoparticles, Inflammation, Uterus, Endothelium Dependent Dilation

Copyright 2018 Alaeddin Bashir Abukabda 


\begin{abstract}
The Microvascular and Systemic Effects of Titanium Dioxide Nanoparticle Exposure: A Physiological Perspective
\end{abstract}

\author{
Alaeddin B. Abukabda
}

Titanium dioxide nanoparticles (nano- $\mathrm{TiO}_{2}$ ) are one of the most widely used engineered nanomaterials (ENM). Their ubiquitous inclusion warrants a more thorough understanding of their toxicity, particularly in sensitive populations such as pregnant women and the developing fetus.

The aim of the first study was to determine the most sensitive segments of the vasculature to pulmonary ENM exposure. Macrovascular function was investigated in the thoracic aorta, common femoral artery and $3^{\text {rd }}$ order mesenteric arterioles using wire myography. Microvascular function was assessed in $4^{\text {th }}$ and $5^{\text {th }}$ order mesenteric arterioles via pressure myography. Nano- $\mathrm{TiO}_{2}$ exposure induced impairments in endotheliumdependent and -independent relaxation in the macrovasculature and dysfunction in the microvasculature. Interestingly, this dysfunction was more robust in the microvessels.

The aims of the second study were: 1) to investigate the uterine microvascular repercussions of pulmonary ENM exposure, 2) to identify the role played by innate lymphoid cells in the inflammatory response to acute nano- $\mathrm{TiO}_{2}$ exposure. This study proposes that group II innate lymphoid cells respond within 4 hours to damage-associated molecular patterns from lung epithelial and innate immune cells in response to intratracheal instillation of nano- $\mathrm{TiO}_{2}$. Furthermore, impairment of endotheliumdependent dilation of the uterine radial arterioles was identified 24 hours post-exposure.

Study 3 aimed to determine the effects of gestational nano- $\mathrm{TiO}_{2}$ exposure on the maternal-fetal circulation. Based on previous research, we predicted that gestational nano- $\mathrm{TiO}_{2}$ inhalation exposure would increase placental vascular resistance and impair endothelium-dependent and -independent dilation of the umbilical microvasculature. 


\section{Acknowledgements}

I decided to leave this section blank until the very end, not because of a lack of people to thank but rather because of an inability to adequately express my gratitude. Ever since I started this journey, I have had the chance and luck to meet extraordinary people whose kindness and support have allowed me to be where I am and who even in the hardest of times have unwaveringly stood by my side. This section will break away from previous dissertations in that all of these acknowledgments are good, positive and from the heart, but I promise not to be overly corny!

My first thanks go to my mentor and "bacon buddy" Dr. Timothy Nurkiewicz. I really believe that had it not been for his mentorship style and constant guidance, I would not have been able to reach the goals I had set out for myself. In the years that I have studied under Dr. Nurkiewicz, he radically changed the way I think and made me appreciate the simplicity and elegance of cardiovascular physiology. He showed me the way a scientist should think and even taught me that there is nothing wrong with committing mistakes as long as you learn from them and use them as lessons for the future. My only regret is having to leave his lab where I spent so much of my time and learned so many things.

My sincere gratitude goes to all my graduate committee members for their time and effort during the past few years. I would like to thank Dr. Brock for helping me with all the paperwork that I forgot about or didn`t know I needed to submit. I thank Dr. Stauber for his constant support and patience throughout the years. I thank Dr. Erdely who, apart 
from being extremely knowledgeable about everything toxicology, also became a great friend someplace along the way. I thank Dr. Cuff for being the "go-to guy" in immunology and for his constant help. Last but not least, I thank Dr. Valentine for his support throughout the years. Overall, my graduate committee was an invaluable resource for my work and development as a scientist.

Special thanks go to my first lab mates in the Nurkiewicz lab; Carroll McBride, Dr. Phoebe Stapleton and Dr. Valerie Minarchick. Without Carroll's constant and priceless help I probably would have annihilated the lab a long time ago. For this reason alone, I think everyone in HSC owes him a debt of gratitude. Jokes aside, Carroll's professional and personal presence and are our morning "therapeutic" venting sessions will be dearly missed. I am thankful to Dr. Stapleton for being my de facto mentor in the lab and for teaching me a lot of the "crazy microvascular" techniques that I so proudly brag about now.

I would also like to thank everyone in the Physiology Department, including the staff, and the students. Each person in this Department has in one way or another contributed to my knowledge and made my time here memorable. I will always look fondly back at my days at WVU. 
Special thanks also go to my brothers and sisters, whom I haven't seen in almost a decade. Even though we are far apart, their love and support has been precious and has helped me recover from my daily misfortunes and the curveballs life has thrown at me.

Twenty-four years ago, the world lost a special person to cancer, Nadia Abukabda. Although a lot of time has passed, she is still dearly missed and hopefully she will be proud of what her little brother has accomplished.

I don't even know where to begin thanking my mom and dad. The selfless love, sacrifice and dedication they showered on us throughout the years is something I still cannot fully fathom nor wrap my head around. Nonetheless, I thank them for every little thing that they have done for me.

During my years at WVU I was blessed with two little babies, Noah and Gabriel. Even though they made things a little bit more complicated, they have taught me that even the simplest things, things that we routinely take for granted, can be an exciting and memorable adventure if you are surrounded by the right people. Noah, I see a lot of myself in you (sorry buddy) and I know that you will be able to achieve whatever you set your mind to. Gabriel, I really have not figured you out yet, but I hope someday you will read this and be proud of your dad. 
Last but not least I wanted to thank my wife, Crystal. I know that this adventure has not always been a positive one for you, but I do promise you that things can and will get better. 


\section{Table of Contents}

Abstract...........................

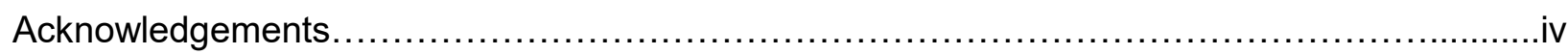

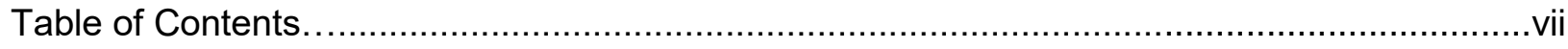

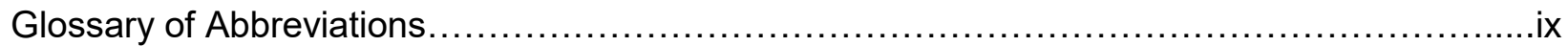

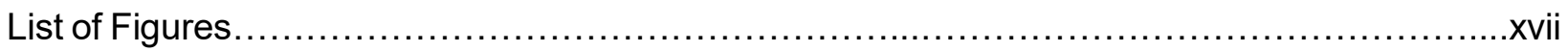

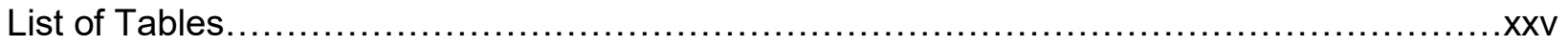

\section{Literature Review}

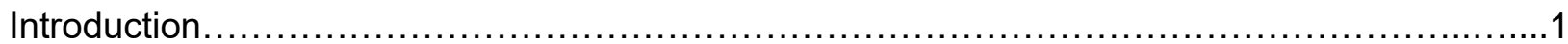

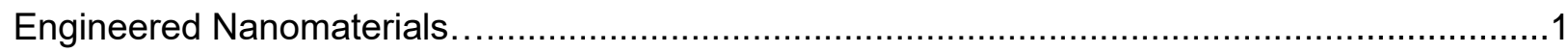

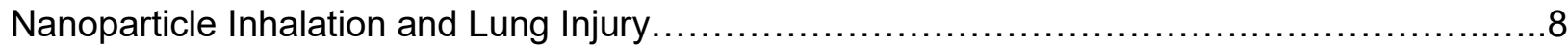

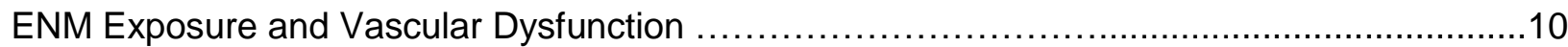

ENM Exposure and Reproductive and Developmental Outcomes..........................22

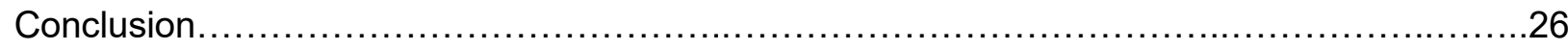

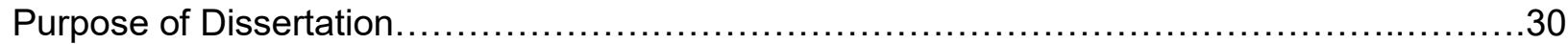

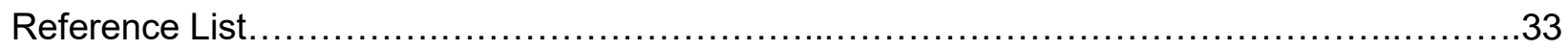

II. Chapter 2: Heterogeneous Vascular Bed Responses to Pulmonary Titanium Dioxide

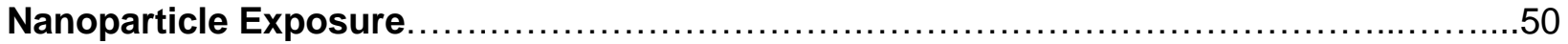

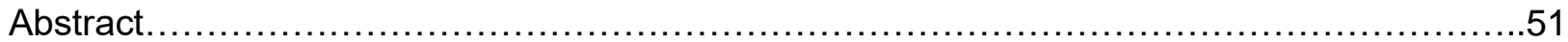

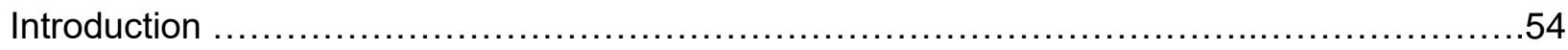

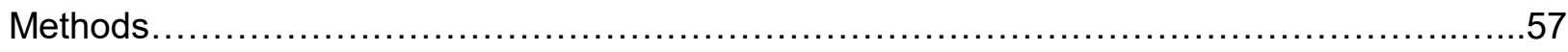

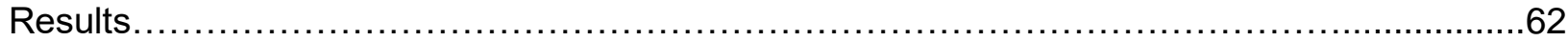

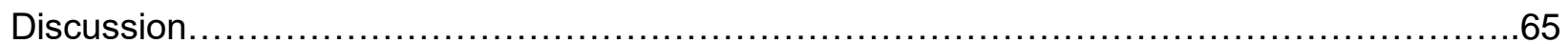

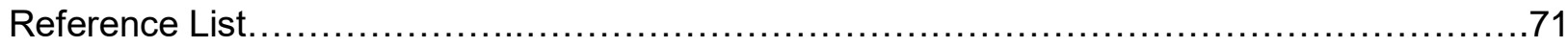

III. Chapter 3: Group II Innate Lymphoid Cells and Microvascular Dysfunction from

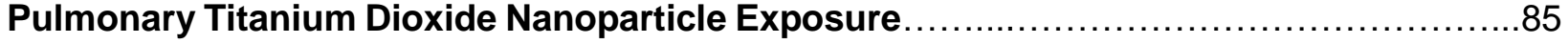

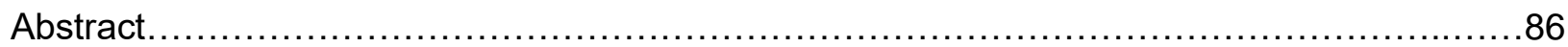

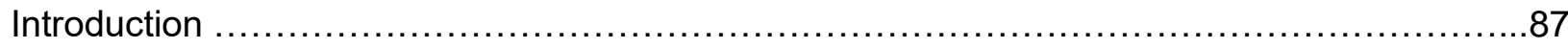

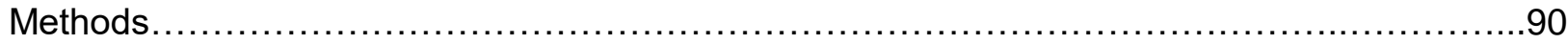

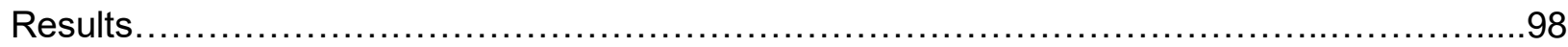

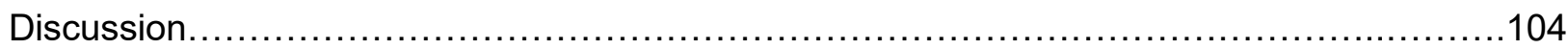

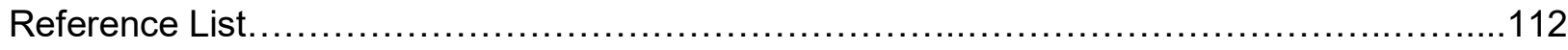


IV. Chapter 4: Maternal Engineered Nanomaterial Inhalation Exposure Compromises

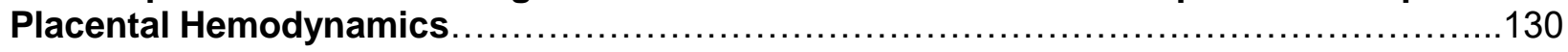

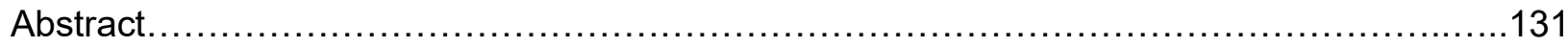

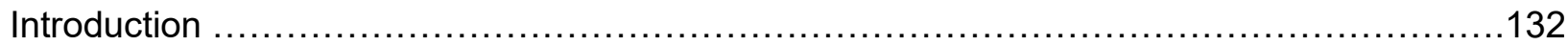

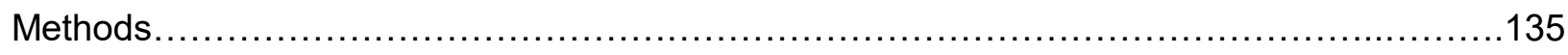

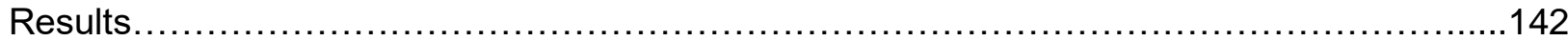

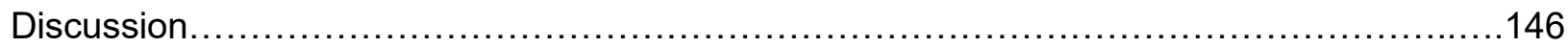

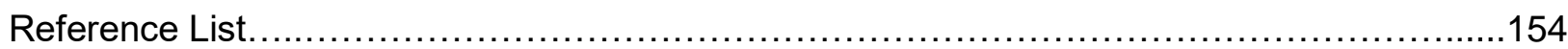

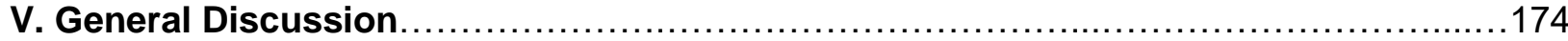

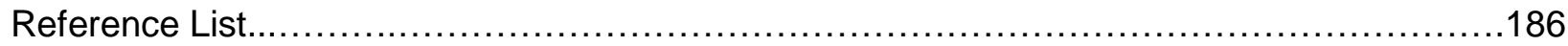

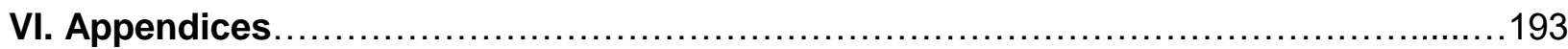

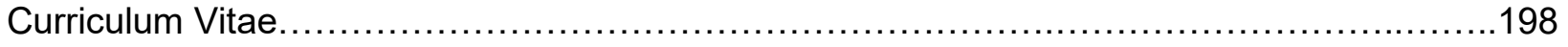




\section{Glossary of Abbreviations}

$\Delta \mathrm{P}$

${ }^{\circ} \mathrm{C}$

$\mu g$

$\mu \mathrm{l}$

$\mathrm{ACh}$

Ag-NP

ANGII

ANOVA

APC

ApoE-/-

APS

Au-NP

BALF

Bax

BBB

Bcl-2

$\mathrm{BH}_{4}$

$\mathrm{BK}_{\mathrm{Ca}}$
Difference in pressure

Degrees Celsius

Micrograms

Microliters

Acetylcholine

Silver nanoparticles

Angiotensin II

Analysis of variance

Allophycocyanin

Apolipoprotein E knockout

Aerodynamic particle sizer

Gold nanoparticles

Bronchoalveolar lavage fluid

Bcl-2-associated X

Blood brain barrier

B-cell lymphoma 2

Tetrahydrobiopterin

Calcium-activated big potassium channels 
C

Ca2+-free

CD

$\mathrm{CeO}_{2}-\mathrm{NP}$

cGMP

coX-2

CuNP

d

D

DAMPs

DAPI

Dcon

Di

Dm

DMT

DNA

Dss

EDTA

ELPI

ENM
Mass concentration

Calcium-free superfusate

Clusters of differentiation

Cerium dioxide nanoparticles

Cyclic guanosine monophosphate

Cyclooxygenase-2

Copper nanoparticles

Diameter

Deposition

Damage-associated molecular patterns

4',6-diamidino-2-phenylindole

Control diameter

Internal diameter

Maximal diameter

Danish MyoTechnologies

Deoxyribonucleic acid

Steady state diameter

Ethylenediaminetetraacetic acid

Electrical low-pressure impactor

Engineered nanomaterials 


\begin{tabular}{|c|c|}
\hline eNOS & Endothelial nitric oxide synthase \\
\hline $\mathrm{F}$ & Fractional deposition \\
\hline FAD & Flavin adenine dinucleotide \\
\hline FeO-NP & Iron oxide nanoparticles \\
\hline FITC & Fluorescein isothiocyanate \\
\hline GD & Gestational day \\
\hline GTP & Guanosine triphosphate \\
\hline HEPA & High-efficiency particulate air \\
\hline HPAG & High-pressure acoustical generator \\
\hline ICAM-1 & Intercellular adhesion molecule-1 \\
\hline ID & Internal diameter \\
\hline IFN-y & Interferon gamma \\
\hline $\lg A$ & Immunoglobulin A \\
\hline $\lg E$ & Immunoglobulin E \\
\hline $\lg G$ & Immunoglobulin G \\
\hline $\lg M$ & Immunoglobulin M \\
\hline IKK & IKB kinase \\
\hline IL-1 $\alpha$ & Interleukin-1 $\alpha$ \\
\hline IL-1 $\beta$ & Interleukin-1 $\beta$ \\
\hline IL-4 & Interleukin-4 \\
\hline
\end{tabular}




\begin{tabular}{|c|c|}
\hline IL-5 & Interleukin-5 \\
\hline IL-8 & Interleukin-8 \\
\hline IL-9 & Interleukin-9 \\
\hline IL-17 & Interleukin-17 \\
\hline IL-22 & Interleukin-22 \\
\hline IL-13 & Interleukin-13 \\
\hline IL-33 & Interleukin-33 \\
\hline ILC & Innate lymphoid cells \\
\hline ILC1 & Group 1 innate lymphoid cells \\
\hline ILC2 & Group 2 innate lymphoid cells \\
\hline IT & Intratracheal instillation \\
\hline IUGR & Intrauterine growth restriction \\
\hline $\mathrm{K}+\mathrm{PSS}$ & High-potassium physiological saline solution \\
\hline KC/GRO & Keratinocyte chemoattractant / human growth-regulated oncogene \\
\hline $\mathrm{KeV}$ & Kiloelectron volt \\
\hline $\mathrm{Kg}$ & Kilogram \\
\hline $\mathrm{kDa}$ & Kilodalton \\
\hline KLRG-1 & Killer Cell Lectin Like Receptor G-1 \\
\hline L & Length \\
\hline Lin & Lineage \\
\hline
\end{tabular}




\begin{tabular}{|c|c|}
\hline M & Molar \\
\hline MAP & Mean arterial pressure \\
\hline MCP-1 & Monocyte chemoattractant protein-1 \\
\hline MCSF & Macrophage colony stimulating factor \\
\hline $\mathrm{mM}$ & Millimolar \\
\hline $\mathrm{mm} \mathrm{Hg}$ & Millimeters of mercury \\
\hline MSNP & Mesoporous silica nanoparticles \\
\hline $\mathrm{mV}$ & Millivolts \\
\hline MWCNT & Multi-walled carbon nanotubes \\
\hline $\mathrm{N}$ & Animal number \\
\hline $\mathrm{n}$ & Vessel number \\
\hline NADPH & Reduced nicotinamide adenine dinucleotide \\
\hline $\mathrm{Nano}^{-\mathrm{TiO}_{2}}$ & Titanium dioxide nanoparticles \\
\hline NF-kB & Nuclear Factor kappa-light-chain-enhancer of activated B cells \\
\hline NGS & Normal goat serum \\
\hline $\mathrm{NIOSH}$ & National Institute for Occupational Safety and Health \\
\hline NK & Natural Killer \\
\hline $\mathrm{nm}$ & Nanometer \\
\hline NO & Nitric oxide \\
\hline NOS & Nitric oxide synthase \\
\hline
\end{tabular}


NOX-1

NRLP3

OD

OSHA

PAI-1

PBS

PBSTX

pDR

PE

PE

PEI

PSL

PSS

Q

R

RAS

ROS

SE

SEM

Ser-536

\section{NADPH Oxidase-1}

Nucleotide-binding domain-like receptor protein 3

Optical density

Occupational Safety and Health Administration

Plasminogen activator inhibitor-1

Phosphate-buffered saline

Phosphate-buffered saline and Triton-X

Personal dataram

Phenylephrine

Phycoerythrin

Polyethyleneimine

Polystyrene test particles

Physiological saline solution

Flow

Resistance

Renin-angiotensin system

Reactive oxygen species

Standard error

Standard error of the mean

Serine-536 
SMA

SMPS

SNAP

SNP

Src

ST2

SWCNT

$\mathrm{T}$

TGF- $\beta$

Th2

THP-1

TIMP-1

$\mathrm{TNF}-\alpha$

TSA

TSP-1

UV

V

VEGF

vWF
Smooth muscle actin

Scanning-mobility particle sizer

S-Nitroso-N-acetyl-DL-penicillamine

Sodium nitroprusside

Sarcoma

Suppression of tumorgenicity

Single-walled carbon nanotube

Exposure time

Transforming growth factor beta

T helper 2

Tamm-Horsfall Protein 1

Tissue inhibitor of metalloproteinases-1

Tumor necrosis factor alpha

Biotinylated tyramine

Thrombospondin-1

Ultraviolet

Minute ventilation

Vascular endothelium growth factor

von Willerbrand factor 
WLR

WT

ZnO-NP
Wall to lumen ratio

Wall thickness

Zinc oxide nanoparticles 


\section{List of Figures}

\section{Literature Review}

Figure 1. Schematic representation of the cardiopulmonary system and identification of locations for ENM influences/activities. Black arrows: blood flow direction. Red represents oxygenated blood. Blue represents deoxygenated blood. 1) Extrapulmonary translocation to systemic circulation. 2) Arterial bifurcation and impaction. 3) Eddy current turbulence and impaction. 4) Segre-Silberg effect and plasma settling. 5) Systemic capillary translocation and tissue deposition. 6) Venular inflammatory signaling. 7) Venous settling. For simplicity, only one systemic tissue is depicted.

Figure 2. Schematic representation of the maternal-fetal circulation in rodents. Diagram showing the maternal-fetal arterial (red) and venous circulation (blue), the direction of blood flow and the location of the trophoblast layer in rodents .50

\section{Chapter 2}

Figure 1. Endothelium - dependent dilation is impaired by intratracheal instillation of nano$\mathrm{TiO}_{2}$. ACh-induced vascular reactivity in A) Aorta, B) Femoral artery, C) $3^{\text {rd }}$ order mesenteric arterioles, and D) $4^{\text {th }}$ and $5^{\text {th }}$ mesenteric arterioles $(n=10-11)$. Statistics were analyzed with twoway ANOVA $(P \leq 0.05)$ * Sham control group vs. nano- $\mathrm{TiO}_{2}$ exposed

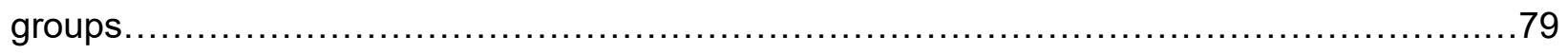

Figure 2. ACh sensitivity and vascular reactivity are decreased by nano- $\mathrm{TiO}_{2}$ exposure. Bar graphs showing the individual slope values for sham control and exposed vessels $(n=9$ - 
11). Statistics were analyzed with two-way ANOVA (P $\leq 0.05)$. * Sham control group vs. nano-

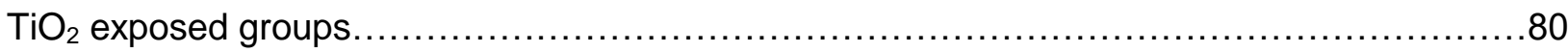

Figure 3. $\alpha$-adrenergic response to $\mathrm{PE}$ is not affected by nano- $\mathrm{TiO}_{2}$. PE response in A) Aorta, B) Femoral artery, C) $3^{\text {rd }}$ order mesenteric arterioles, and D) $4^{\text {th }}$ and $5^{\text {th }}$ mesenteric arterioles $(n=$ 10-11). Statistics were analyzed with two-way ANOVA (P $\leq 0.05)$. * Sham control group vs. nano$\mathrm{TiO}_{2}$ exposed groups .81

Figure 4. $\mathrm{PE}$ sensitivity are augmented by nano- $\mathrm{TiO}_{2}$ exposure. Bar graphs showing the individual slope values for sham-control and exposed vessels $(n=10-11)$. Statistics were analyzed with two-way ANOVA ( $\mathrm{P} \leq 0.05)$. * Sham control group vs. nano- $\mathrm{TiO}_{2}$ exposed groups .82

Figure 5. Endothelium-Independent dilation and vascular smooth muscle function is not impaired by nano- $\mathrm{TiO}_{2}$ exposure. SNP response in A) Aorta, B) Femoral artery, C) $3^{\text {rd }}$ order mesenteric arterioles, and D) $4^{\text {th }}$ and $5^{\text {th }}$ mesenteric arterioles $(n=10-11)$. Statistics were analyzed with two-way ANOVA $(\mathrm{P} \leq 0.05)$. * Sham control group vs. nano- $\mathrm{TiO}_{2}$ exposed groups.

Figure 6. SNP-induced vascular reactivity is unaffected by nano- $\mathrm{TiO}_{2}$ exposure. Bar graphs showing the individual slope values for sham-control and exposed vessels $(n=10-11)$. Statistics were analyzed with two-way ANOVA $(\mathrm{P} \leq 0.05)$. * Sham control group vs. nano-TiO 2 exposed groups .84 


\section{Chapter 3}

Figure 1. Characterization of Nano- $\mathrm{TiO}_{2}$. (A) SEM image and (B) Energy dispersive spectroscopy showing elemental composition of the nano- $\mathrm{TiO}_{2}$ suspension used in this study..120

Figure 2. Nano- $\mathrm{TiO}_{2}$ exposure increases $\mathrm{T}$-Helper type II cytokines 4 hours post-exposure. Multiplex cytokine analysis showing concentrations of (A) IL-4, (B) IL-1 $\beta$, (C) TNF- $\alpha$, (D) IL-5, and (E) IL-13. Serum samples were obtained at 0,1,2 and 4 hours post-exposure via tail-vein puncture $(N=6)$. Statistics were analyzed with two-way ANOVA $(P \leq 0.05),{ }^{*}$ Sham control group vs. nano- $\mathrm{TiO}_{2}$ exposed groups

Figure 3. Nano- $\mathrm{TiO}_{2}$ exposure also increases $\mathrm{T}$-Helper type II cytokines 4 hours postexposure in bronchoalveolar lavage fluid (BALF). Multiplex cytokine analysis showing concentrations of IL-4, IL-5, IL-13 in BALF from exposed and control animals 4 hours postexposure $(\mathrm{N}=6)$. Statistics were analyzed with two-way ANOVA $(\mathrm{P} \leq 0.05)$, ${ }^{*}$ Sham control group vs. nano- $\mathrm{TiO}_{2}$ exposed groups 121

Figure 4. Nano- $\mathrm{TiO}_{2}$ exposure is associated with an increase in pulmonary interleukin-33 levels. Lung sections from control (A) and nano- $\mathrm{TiO}_{2}$ exposed (B) animals obtained 4 hours postexposure were stained for interleukin-33. Fluorescence was achieved by staining tracheal sections with an anti-IL-33-FITC conjugated antibody. Relative fluorescence is shown in (C) tagged antibody $(\mathrm{N}=6-7)$. Statistics were analyzed with two-way ANOVA $(\mathrm{P} \leq 0.05)$, * Sham control group vs. nano- $\mathrm{TiO}_{2}$ exposed groups

Figure 5. Pre-treatment of nano- $\mathrm{TiO}_{2}$ - exposed animals with an anti-IL-33 antibody lowers BALF and plasma IL-33. Interleukin-33 levels in (A) BALF and (B) plasma in control, nano- $\mathrm{TiO}_{2}$ 
exposed groups and in rats pre-treated with an anti-IL-33 antibody. Rats were pre-treated with an anti-IL-33 antibody (1 mg/kg) 30 minutes prior to exposure. Plasma and BALF IL-33 levels were measured 4 hours post-exposure. Statistics were analyzed with two-way ANOVA (P $\leq 0.05)$. * Sham control group vs. nano- $\mathrm{TiO}_{2}$ exposed groups, $\mp \mathrm{P}<0.05$ Exposed + Anti-IL-33 Antibody vs Exposed. 123

Figure 6. Flow cytometric analysis of lung-resident Group II innate lymphoid cells. Flow cytometry of lung tissue 4 hours post-exposure for Group II Innate Lymphoid Cells in (A) control and $(\mathrm{B})$ nano- $\mathrm{TiO}_{2}$ exposed animals $(\mathrm{N}=6)$. 124

Figure 7. Nano- $\mathrm{TiO}_{2}$ exposure increases pulmonary ILC2 but not ILC1. Quantification of percentage of total pulmonary cells of (A) ILC1 and (B) ILC2 in control and nano- $\mathrm{TiO}_{2}$ exposed animals $(\mathrm{N}=6)$. Statistics were analyzed with two-way ANOVA $(\mathrm{P} \leq 0.05),{ }^{*}$ Sham control group vs. nano- $\mathrm{TiO}_{2}$ exposed groups. .125

Figure 8. Nano- $\mathrm{TiO}_{2}$ exposure increases NF-kB phosphorylation at Serine-468 in isolated ILC2. Quantification of NF-kB phosphorylation at (A) serine-468 and (B) serine 536 in isolated ILC2 from control and nano- $\mathrm{TiO}_{2}$ exposed animals $(\mathrm{N}=6)$. Statistics were analyzed with two-way ANOVA $(\mathrm{P} \leq 0.05),{ }^{*}$ Sham control group vs. nano- $\mathrm{TiO}_{2}$ exposed groups

Figure 9. Th2 cytokine secretion in isolated ILC2 is increased by nano- $\mathrm{TiO}_{2}$ exposure. Multiplex cytokine analysis showing concentrations of IL-4, IL-5, IL-13 supernatant from isolated ILC2 cultured overnight from control and nano- $\mathrm{TiO}_{2}$ exposed animals $(\mathrm{N}=6)$. Statistics were analyzed with two-way ANOVA (P $\leq 0.05)$, * Sham control group vs. nano- $\mathrm{TiO}_{2}$ exposed groups. 127 
Figure 10. Endothelium-dependent dilation of radial arterioles is blunted by nano- $\mathrm{TiO}_{2}$ exposure and improved by pre-treatment with an anti-IL-33 antibody. Endotheliumdependent dilation of uterine radial arterioles from control, exposed and anti-IL-33 antibody treated animals was determined using pressure myography $(n=12-18)$. All vascular assessments were performed 24 hours post-exposure. Statistics were analyzed with two-way ANOVA ( $\mathrm{P} \leq$ 0.05). * Sham control group vs. nano- $\mathrm{TiO}_{2}$ exposed groups, $\mp \mathrm{P}<0.05$ Exposed + Anti-IL-33 Antibody vs Exposed. .128

\section{Chapter 4}

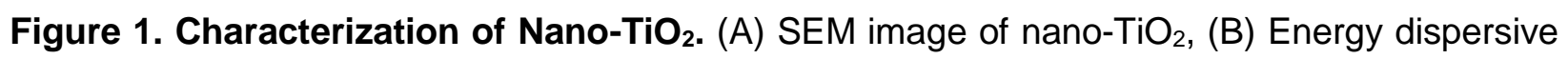
spectroscopy showing elemental composition of the nano- $\mathrm{TiO}_{2}$ suspension used in this study, (C) Nano- $\mathrm{TiO}_{2}$ size distribution (mobility diameter, $190 \mathrm{~nm}$ ) using a scanning mobility particle sizer (SMPS), (D) Nano- $\mathrm{TiO}_{2}$ size distribution (aerodynamic diameter, $188 \mathrm{~nm}$ ) using a high resolution electrical low-pressure impactor (ELPI). (E) Total aerosol concentration $\left(10 \mathrm{mg} / \mathrm{m}^{3}\right)$ of engineered nano- $\mathrm{TiO}_{2}$ during maternal exposures .164

Figure 2. Ex vivo placental perfusion preparation. (A) Umbilical artery (1); vein (2). (B) Input (arterial) perfusion pressure (1) $(0-80 \mathrm{~mm} \mathrm{Hg})$ is held constant while vasoactive agonists are added. Output pressure (2) and flow (3) ( $\mu \mathrm{l} / \mathrm{min})$ are variables responsive to placental resistance. Vascular diameter may be measured with video calipers. The chamber is designed for inverted microscopy, and fluorescent biomarkers may be easily added to the perfusate or superfusate...165

Figure 3. $\mathrm{Nano}^{-\mathrm{TiO}_{2}}$ inhalation exposure impairs baseline and endothelial-dependent placental hemodynamics. Mean outflow pressures were measured in sham-control and nano- 
$\mathrm{TiO}_{2}$ exposed animals after the inflow pressure was increased in a stepwise manner and set at 0 mm Hg, 20 mm Hg, 40 mm Hg, 60 mm Hg and 80 mm Hg. Nano-TiO 2 inhalation exposure altered placental hemodynamics and decreased outflow (venous) pressure in placentas in normal superfusate (A) and placentas treated with $A C h(B)(n=8) .{ }^{*}, P \leq 0.05$ sham-control group vs. nano- $\mathrm{TiO}_{2}$ exposed group. 166

Figure 4. Nano- $\mathrm{TiO}_{2}$ inhalation exposure does not affect endothelial-independent placental hemodynamics and increases ANGIl sensitivity. Mean outflow pressures were measured in sham-control and nano- $\mathrm{TiO}_{2}$ exposed animals after the inflow pressure was increased in a stepwise manner and set at $0 \mathrm{~mm} \mathrm{Hg}, 20 \mathrm{~mm} \mathrm{Hg}, 40 \mathrm{~mm} \mathrm{Hg}, 60 \mathrm{~mm} \mathrm{Hg}$ and $80 \mathrm{~mm} \mathrm{Hg}$. Maternal nano-TiO2 inhalation exposure did not affect endothelial-independent placental hemodynamics (A) but increased ANGIl sensitivity (B) $(n=8)$. *, $\mathrm{P} \leq 0.05$ sham-control group vs. nano- $\mathrm{TiO}_{2}$ exposed group. 166

Figure 5. Nano- $\mathrm{TiO}_{2}$ inhalation impairs calcium-free placental hemodynamics. Mean outflow pressures were measured in sham-control and nano- $\mathrm{TiO}_{2}$ exposed animals after the inflow pressure was increased in a stepwise manner and set at $0 \mathrm{~mm} \mathrm{Hg}, 20 \mathrm{~mm} \mathrm{Hg}, 40 \mathrm{~mm} \mathrm{Hg}, 60$ $\mathrm{mm} \mathrm{Hg}$ and $80 \mathrm{~mm} \mathrm{Hg}$. Maternal nano- $\mathrm{TiO}_{2}$ inhalation exposure altered placental hemodynamics and decreased the outflow pressure in placentas placed in calcium-free superfusate $(n=8)$. *, P $\leq$ 0.05 sham-control group vs. nano- $\mathrm{TiO}_{2}$ exposed group 167

Figure 6. Nano- $\mathrm{TiO}_{2}$ inhalation exposure and placental mean outflow pressure. Mean outflow pressure at $80 \mathrm{~mm} \mathrm{Hg}$ inflow pressure in both Sham-control and nano- $\mathrm{TiO}_{2}$ exposed 
groups and all treatment conditions is shown $(n=8)$. Statistics were analyzed with a student's ttest $(\mathrm{P} \leq 0.05),{ }^{*}$ Sham control group vs. nano- $\mathrm{TiO}_{2}$ exposed groups. .168

Figure 7. Maternal nano- $\mathrm{TiO}_{2}$ inhalation exposure impairs endothelium-dependent dilation of the umbilical artery and vein. (A) Endothelium-dependent dilation of the umbilical artery, (B) endothelium-dependent dilation of the umbilical vein from Sham-control and nano- $\mathrm{TiO}_{2}$ exposed animals was determined using pressure myography $(n=12-14)$. Statistics were analyzed with twoway ANOVA (P $\leq 0.05)$ * Sham control group vs. nano- $\mathrm{TiO}_{2}$ exposed group.... 169

Figure 8. Maternal nano- $\mathrm{TiO}_{2}$ inhalation exposure impairs endothelium-independent dilation of the umbilical artery and vein. $(A)$ Endothelium-independent dilation of the umbilical artery, (B) endothelium-independent dilation of the umbilical vein from Sham-control and nano$\mathrm{TiO}_{2}$ exposed animals was determined using pressure myography $(n=12-14)$. Statistics were analyzed with two-way ANOVA $(\mathrm{P} \leq 0.05)$. * Sham control group vs. nano- $\mathrm{TiO}_{2}$ exposed group

Figure 9. Maternal nano- $\mathrm{TiO}_{2}$ inhalation exposure increases ANGIl sensitivity of the umbilical artery. (A) ANGII dose-response curve of the umbilical artery (A) and vein (B) from Sham-control and nano- $\mathrm{TiO}_{2}$ exposed animals was determined using pressure myography $(\mathrm{n}=12$ 14). Statistics were analyzed with two-way ANOVA $(P \leq 0.05) .{ }^{*}$ Sham control group vs. nano$\mathrm{TiO}_{2}$ exposed group.

Figure 10. Figure 10. Placental immunohistochemistry. Smooth muscle actin is green, VonWillebrand Factor is red, and DAPI nuclear staining is blue. (A) Representative image showing immunohistochemistry of a placental section from maternal nano- $\mathrm{TiO}_{2}$ exposed animal. The purpose was to identify the microvascular architecture and the structural components of the xxiii 
placenta. (B) An enlarged (40x) image of a placental microvessel displaying an intact endothelium

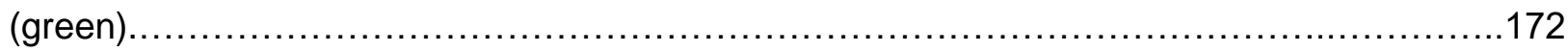




\section{List of Tables}

\section{Chapter 2}

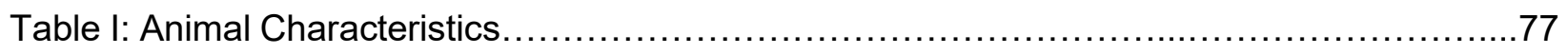

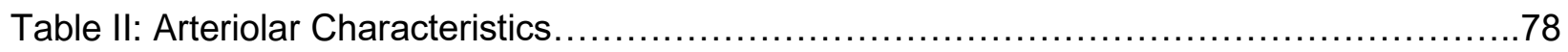

\section{Chapter 3}

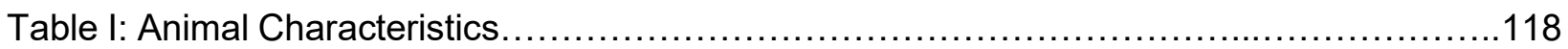

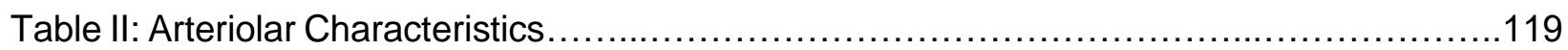

\section{Chapter 4}

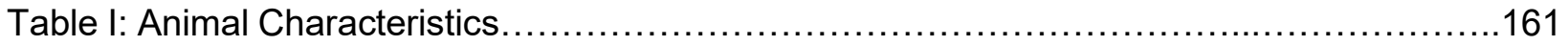

Table II: Umbilical Artery Characteristics..................................................

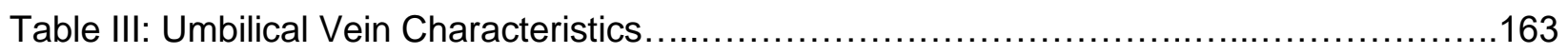




\section{Literature Review}

With the introduction of novel man-made materials consisting of particles with at least one dimension smaller less than $100 \mathrm{~nm}$ called engineered nanomaterials (ENM), unique health hazards resulting from exposure to such materials has been identified [91]. Of the various possible routes of entry into the body, the airway seems to be the easiest entry for ENM. After arrival into the lungs, local lung injury can occur from nanoparticles [59]. In addition, effects can be seen systemically in the cardiovascular system resulting from the effects of lung inflammation perhaps mediated by inflammatory cytokines [91]. Furthermore, inhaled ENM can be transported to other organs by the cardiovascular system [62]. Because of intravascular transport, the cardiovascular system can also become diseased with reduced ability to vasodilate as one of the hallmarks of impaired function and disease risk [91]. However, it is not known if all parts of the cardiovascular system are equally impaired. Since both the uterine and fetal circulation possess unique adaptations [118], the fetal and placental circulation might be protected from the adverse effects of ENM exposure on the maternal circulation. If not, then what is the outcome of ENM exposure in the lung to the maternal-fetal exchange of nutrients?

\section{Engineered Nanomaterials}

A. Introduction. The demand for diverse materials with novel properties has led to the advancement of the nanotechnology field. The unique physicochemical characteristics that specific materials exhibit at the nanoscale make them highly desirable and versatile. Indeed, the National Nanotechnology Initiative defined the term Nanotechnology as "the understanding and control of matter at dimensions between approximately 1 and $100 \mathrm{~nm}$, where unique phenomena 
enable novel applications" [85]. While it is apparent that nanotechnology holds tremendous potential benefits, there are also significant risks to human health as the biological effects caused by exposure are not adequately understood. For example, effects of particle exposure on human health, particularly the cardiovascular system have been reported [106]. Several mechanisms have been proposed to account for the increase in morbidity including; oxidative stress, systemic inflammation, endothelial dysfunction, thrombosis and arrhythmia [88,142] following human exposure.

B. Properties of Engineered Nanomaterials. The physicochemical properties of ENM are fundamental determinants of their biodistribution and biokinetics $[63,142]$. The nanoscale size of ENM results in a significantly greater surface area which leads to chemical reactivity that differs from those of the corresponding bulk materials. Therefore, material characterization is necessary to determine any potential toxic effects.

i) Size. The most fundamental ENM physical property determining pulmonary deposition, extrapulmonary translocation, and cellular uptake is size [64,96]. ENM particles show a great tendency to agglomerate into larger structures based on surface electrical potential and particle diameter [73]. This agglomeration has a significant impact on the deposition pattern of ENM within the respiratory tract [127].

The ability to enter the systemic circulation and the translocation pattern of ENM is correlated with particle diameter [62]. ENM have extended circulating half-lives and are cleared less efficiently by phagocytic cells than particles $>100 \mathrm{~nm}$. Ten nm gold nanoparticles (AuNP) translocated from the blood to spleen, kidney, liver, testes, thymus, heart, lung, while larger particles (50 nm, 100 
$\mathrm{nm}$, and $200 \mathrm{~nm}$ ) were present predominantly in the liver and spleen [29]. ENM smaller than serum albumin (40-50 kDa, approximate diameter 4-6 nm) are eliminated primarily through the kidneys [10, 31]. Elimination occurs because of the selectivity of the glomerular filtration barrier that restricts the passage of molecules according to size, shape and charge $[13,14]$. ENM within the kidney may therefore avoid ultrafiltration, persist within the circulation, and translocate to other organs, such as the liver and spleen. Secondary destinations of ENM include the blood conditioning organs with major homeostatic functions (liver, kidney, spleen). The accumulation of ENM in these organs may simply be due to the significant portion of the cardiac output these organs receive.

ii) Charge and Surface Chemistry. The surface properties of ENM are a direct product of their size. The decrease in atomic radius occurring in nanoscale materials increases the energy gap between electron transition states, resulting in an increased surface reactivity. This phenomenon, known as quantum confinement, accounts for the difference in chemical reactivity between ENM and bulk materials [138]. The surface properties of metals and metal oxides are influenced by the presence of defects in their crystalline structures, adsorbed ions, and functional groups. Carbonaceous ENM surface chemistry is determined primarily by the degree of bonding or hybridization state of the individual carbon atoms [76]. The increased reactivity may favor the interaction of ENM with biological molecules, subcellular components and the immune system.

Functional groups are specific moieties within molecules responsible for the chemical identity of those molecules. The addition of these chemical groups (e.g. carboxylate, amines etc.) can significantly modify the reactivity of ENM. Surface functionalization may influence biodistribution [63], cellular uptake [23], and toxicity [74]. 
The cell membrane possesses a net negative charge because of the presence of anionic residues such as proteins, phospholipids and glycoproteins. The plasma membrane also contains discrete microdomains of glycolipids and proteins known as lipid rafts. The localized distribution of these negatively-charged biomolecules may selectively dictate the interaction with ENM based on surface charge and chemical composition. Therefore, ENM with cationic chemical groups are frequently used to promote cellular adsorption and uptake [24]. Mesoporous silica nanoparticles (MSNP) coated with cationic polyethyleneimine (PEI) undergo greater cellular uptake than unmodified MSNP [136]. Intravenously administered positively charged AuNP showed greater adsorption to the cell membrane of circulating red blood cells than neutral or negatively charged AuNP [49].

Surface chemistry may also determine the distribution of ENM to specific target organs and the inflammatory response to ENM exposure. Intravenous administration of $2.8 \mathrm{~nm}$ AuNP coated with negatively charged thioglycolic acid accumulated preferentially within the liver, while positively charged AuNP showed higher circulating and kidney concentrations [49]. Ultrafine 60$\mathrm{nm}$ polystyrene-test particles (PSL) with a positive surface charge increased the tendency of blood to clot compared to controls and carboxyl-coated PSL [87]. Charged (positive and negative) nanoparticles elicit a more robust response by the immune system, undergo greater opsonization and activate phagocytosis by cells of the mononuclear phagocytic system [25]. Furthermore, multiwall carbon nanotubes (MWCNT) functionalized with PEI induced a greater NRLP3 inflammasome activation and IL-1 $\beta$ release in vitro by THP-1 cells than pristine and negatively charged MWCNT [69]. In addition, activation of immunological pathways may result in the systemic release of proinflammatory cytokines and oxidative stress, resulting in vascular dysfunction [91]. 
Historically, addition of functional groups has been employed to increase ENM circulation time and maintain concentrations for therapeutic use. Amphiphilic polymer coatings are effective in reducing first-pass metabolism by the liver [54]. Increasing surface hydrophobicity by coating liposomes with erythrocytic sialic acid moieties reduced opsonization and clearance by phagocytic cells [5]. Coating nanoparticles with polysaccharides such as dextran [86] and polyethylene glycol [59] increased plasma half-life by decreasing activation of innate immunity.

The osmolarity, $\mathrm{pH}$, and charge of specific intracellular and extracellular compartments can also impact ENM surface chemistry [51]. When suspended in a liquid phase, ENM form a colloidal system. The stability of this system and the interaction with cellular components can be assessed by measuring the zeta potential of ENM [141]. The zeta potential predicts the electro kinetic behavior of particles in solution and is a measure of the electrostatic attraction or repulsion between ENM particles [34]. Metal oxides show an increased agglomeration in $\mathrm{pH}$ environments approaching their isoelectric point [107]. The increase in particle size caused by agglomeration may decrease renal clearance and increase ENM plasma half-life. This could increase the likelihood of deposition within the vascular wall and compromise cardiovascular health.

When ENM enter physiological compartments, a complex mixture of proteins and biomolecules may bind non-specifically to their surface. The composition of this adsorbed coat, also known as protein corona, is influenced by ENM surface chemistry [4,102]. The dynamics of this interaction are defined by ENM physicochemical properties [111]. The acquisition of a distinct protein corona may influence the 'biological identity', cellular uptake, and the immunogenicity of ENM $[33,129]$. In a recent study, the adsorption of serum proteins to AuNP was inversely dependent on size [129]. Plasma IgG showed a higher affinity for $100 \mathrm{~nm}$ and $50 \mathrm{~nm}$ plain PSL nanoparticles, and 
little binding to carboxyl- and amine- modified $50 \mathrm{~nm}$ PSL nanoparticles [74]. The formation of a protein corona has been shown to increase macrophage phagocytic activity [129] and elicit a more pronounced innate immune response. Further, it may also enhance ENM biocompatibility (38), and regulate their impact on the cardiovascular system.

iii) Shape. Recent studies have highlighted the importance of ENM morphology in the interaction with biological systems. ENM with a high length to diameter ratio or aspect ratio (nanorods, nanobelts, nanowires and nanotubes) may pierce cellular membranes and disrupt anatomical barriers. ENM with high curvature angles resist uptake by phagocytic cells, particularly macrophages [20]. MWCNT with a low aspect ratio (220 nm length and $25 \mathrm{~nm}$ diameter) administered subcutaneously in rats persisted within the cytosol of macrophages for more than 4 weeks. High aspect ratio MWCNT (825 nm length and $25 \mathrm{~nm}$ diameter) were not phagocytized and triggered a robust inflammatory response [109]. Ellipsoid particles are more readily phagocytized by cells of the immune system than spherical particles [112]. Gold nanorods of greater aspect ratio are not only internalized slower than shorter ones but are more toxic to human HeCaT keratinocytes [130]. The inability to efficiently clear ENM by the immune system may result in frustrated phagocytosis and pro-fibrotic cellular responses [130] including the release of proinflammatory chemokines that may lead to peripheral vascular consequences.

ENM aspect ratio must be considered when determining potential vascular effects. ENM with high aspect ratios (nanotubes, nanobelts and nanowires) are more likely to interrupt laminar flow, deposit in the vascular wall and damage endothelial or vascular smooth muscle cells. ENM may also interact with endothelial cells under conditions of turbulent flow or in bifurcations. High aspect ratio fibers may cause occlusion of microvessels and promote thrombus formation. 
iii) Chemical composition. Chemical composition remains the primary factor for risk from ENM [17]. Therefore, the primary focus of this review will be on the most common nanomaterials subdivided according to chemical composition: metals, metal oxides, and carbonaceous nanomaterials.

iv) Routes of entry. The exposure route greatly influences the overall toxicity and the amount of ENM deposition, translocation and interaction with tissues, cells or specific intracellular components. Historically, considering the ease with which ENM can be aerosolized and inhaled, the most frequently studied exposure route has been the pulmonary route. Recent developments in ENM-based therapies and their ubiquitous presence, other routes such as ocular, gastric, dermal and injection are becoming ever more important and should not be overlooked.

a) Inhalation. Inhalation is the most common and the most investigated exposure route. Experimentally, three exposure methods for lung deposition of particulate matter are commonly employed: inhalation, intratracheal instillation and oropharyngeal aspiration. Inhalation creates an artificial atmosphere saturated with particulate matter. Particle deposition is then driven by physiological processes and thermodynamic phenomena. While generally considered as the "golden standard" for pulmonary exposure studies, whole-body inhalation requires significant amounts of material, trained personnel and costly equipment. The two major systems used for inhalation exposures involve whole body inhalation and head/nose-only. Whole body inhalation requires large amounts of bulk material and may result in dermal or oral exposure but has the advantage of allowing the simultaneous exposure of multiple animals for extended periods of time. Nose-only systems limit exposure via other routes by employing a restraint inserted into a 
plenum. Even though this technique is more efficient and requires less bulk material, the restraints may cause significant stress to the animals $[120,133]$.

b) Instillation. Instillation involves lightly anesthetizing the animal and administering particles suspended in a medium directly into the lungs via a ball tipped needle. Instillation is simpler than inhalation (even though some training is required to avoid accidentally gavaging the animals), less expensive and provides a consistent lung deposition. However, particle instillation is an artificial mode of delivery of particulate matter to the lungs. Rats and mice are obligate nose breathers and intratracheal instillation bypasses the nose and upper airways. Therefore, the lung deposition may be substantially different compared to whole-body inhalation. However, it has been shown that even though the dose rate between inhalation and instillation may be different, the pulmonary distribution and biological effects are similar if the delivered dose is equivalent [6].

2. Nanoparticle inhalation and lung injury,

\section{A. Size and location}

The aerodynamic behavior and therefore deposition of particulate matter in the different regions of the pulmonary tract is highly dependent on size [62]. Due to size, ENM deposit throughout the lungs by diffusion and their deposition in the distal or alveolar region increases significantly as particle size decreases. The converse is true for larger particles that deposit in the lungs by means of sedimentation and impaction. Generally, larger particles and agglomerates tend to deposit in the upper, tracheobronchial regions; while ENM tend to deposit throughout the entire lung, including the alveolar region.

B. Cell injury and inflammation 
Inflammation is a well-established consequence of pulmonary ENM exposure. For example, 90day exposure to MWCNT resulted in a concentration-dependent multifocal granulomatous inflammation, diffuse histiocytic and neutrophilic inflammation and intra-alveolar lipoproteinosis, indicative of lung damage oxidative stress [109]. Furthermore, rat intratracheal instillation of zinc oxide nanoparticles (ZnO-NP) induced eosinophilia, proliferation of airway epithelial cells and goblet hyperplasia 24 hours post-exposure [135]. Short-term inhalation exposure (2.5 and 12.5 $\mathrm{mg} / \mathrm{m}^{3}-6 \mathrm{~h} /$ day exposure for 5 days) of coated $\mathrm{ZnO}-\mathrm{NP}$ increased bronchoalveolar lavage fluid inflammatory parameters, resulted in a reversible granulocyte infiltration in the lungs, increased alveolar macrophages and induced a mild to moderate multifocal necrosis of the olfactory epithelium [135]. Sub-chronic inhalation exposure (5 days) of rats to silver nanoparticles (Ag-NP) induced chronic alveolar inflammation and small granulomatous pulmonary lesions [44]. Ceriumdioxide nanoparticles $\left(\mathrm{CeO}_{2}-\mathrm{NP}\right)$ have also been shown to induce pulmonary inflammatory reactions and toxicity [78]. Four-day (2 h/day) inhalation of uncoated $\mathrm{CeO}_{2}-\mathrm{NP}$ with a primary particle size of $90 \mathrm{~nm}$ induced lung injury and inflammation with increased neutrophil infiltration and elevated levels of the pro-inflammatory cytokines $\mathrm{IL}-1 \alpha$, monocyte chemotactic protein 1 (MCP-1), macrophage colony stimulating factor (MCSF) in lung tissue. Mild but persistent diffuse histiocytosis was also reported. Lastly, the inflammatory effects of pulmonary exposure to titanium dioxide nanoparticles $\left(\right.$ nano- $-\mathrm{TiO}_{2}$ ) have also been extensively investigated [89]. Two-year inhalation exposure ( $6 \mathrm{~h} /$ day, 5 days/week) of rats to nano- $\mathrm{TiO}_{2}$ induced pulmonary induced increased levels of pro-inflammatory cytokines in the bronchoalveolar lavage fluid, bronchioalveolar adenomas and cystic keratinizing squamous cell carcinomas in a dosedependent manner [89]. The complex effects of pulmonary ENM exposure on both the innate and adaptive branches of the immune system require further studies on the role of other immune effector cells (e.g., innate lymphoid cells, lymphocytes etc.). 
3. ENM exposure and vascular dysfunction

The cardiovascular system is composed of a central pump (the heart) and a closed system of conduits responsible for transport and distribution of nutrients and waste removal. In attempting to predict the effects of ENM exposure on this system several factors must be considered. First, the distribution of ENM within each vascular segment as a result of direct introduction of ENM in the vascular system or indirectly through other routes depends upon the physicochemical characteristics of the nanomaterial and the dominant physical forces. Second, the severity of the effects of ENM deposition are dictated by the function of each vascular segment (Figure 1). The entity and magnitude of the dominant physical forces in each vascular segment are at the root of the structural heterogeneity of the circulatory system. These forces may account for the distribution and impact of ENM within each vascular segment [119].

A) Microcirculation. The microcirculation is defined as all the vascular tissue within a given organ, which includes arterioles, the capillary network, venules and part of the lymphatic system [104]. The essential function of the microcirculation is the maintenance of an ideal environment for the controlled exchange of nutrients and metabolic waste. For this exchange to occur efficiently, a high surface area and resistance to flow are necessary. Arterioles are involved in the regulation of blood flow and pressure. The arteriolar and venular segments of the microcirculation are under a state of continuous basal contraction known as vascular tone. This baseline tension is mediated by vascular smooth muscle cells. Adjacent to the vascular smooth muscle cells are the endothelial cells, which detect changes in the intraluminal environment. Coordinated interaction between vascular smooth muscle cells and endothelial cells allows for alterations in vascular tone in response to localized environmental changes [9]. 
The local characteristics specific to the microcirculation may lead to a diffused distribution of ENM throughout tissues. A greater tendency towards laminar flow is seen at a threshold vessel diameter of $\sim 300 \mu \mathrm{m}$ [119]. Along with a decreased blood flow and pressure, favors ENM to settle within the microvascular compartment. In conditions of laminar flow, the Segre - Silberberg effect is used to describe parabolic flow pattern of colloidal suspensions. Erythrocytes travelling at higher flow velocities remain in the flow centerline, while smaller particles (including ENM) and non-cellular elements are forced laterally towards the vascular wall [103].

Blood consists of a solid phase (formed elements) suspended in a liquid phase (plasma). This biphasic composition causes blood to behave as a non-Newtonian fluid in the microvasculature. A Newtonian fluid characteristically maintains constant viscosity with increasing shear rate. Blood exhibits shear thinning (or Fahraeus effect) in microvessels and hematocrit (or volume percentage of erythrocytes) gradually decreases towards the capillaries. The decrease in hematocrit seen as capillaries are approached is a result of the branching vascular architecture and increased resistance. At bifurcations the distribution of erythrocytes between two daughter branches is often unequal and may result in one branch receiving a lower proportion of cells relative to the other daughter branch [105]. ENM concentrations may therefore increase with decreasing vessel diameter.

The venous compartment is responsible for returning blood from the systemic organs to the heart and for storage of blood due to its high capacitance. Since about $65 \%$ of blood volume is localized in veins, a significant proportion of ENM may be found here [9]. 


\section{B) Examples of vascular ENM toxicity.}

\section{i) Metallic ENM}

a) Gold Nanoparticles. AuNP have been historically used in medicine as therapeutic agents and are now also utilized in biological imaging and as drug delivery systems. Their bioinert character coupled with a high biocompatibility has fostered significant interest in their implementation as both a therapeutic and diagnostic (theranostic) agent. Their optical properties, characterized by enhanced absorption and scattering at specific electromagnetic wavelengths, have contributed to the application of AuNP for cancer imaging [80].

In a porcine model of the blood brain barrier (BBB), brain microvascular endothelial cells treated with 3 and $5 \mathrm{~nm}$ AuNP displayed an increase in vascular permeability but no change in systemic levels of the proinflammatory cytokines TNF $-\alpha$ and IL- $1 \beta$ [123]. Wistar - Kyoto rats infused with different sizes $(10 \mathrm{~nm}-100 \mathrm{~nm})$ of AuNP presented hepatic leukocytic infiltration, and disruption of the central vein intima in a size-dependent manner [2]. Damage to the venous wall could potentially lead to impaired capacitance as well as a decreased venous return.

Gold nanorods functionalized with polyelectrolytes induced cytotoxicity of aortic vascular endothelium and impaired endothelium-dependent relaxation of aortic rings [3]. In a separate study, $5 \mathrm{~nm}$ AuNP were shown to hyperpolarize aortic vascular smooth muscle due to activation of $\mathrm{BK}_{\mathrm{Ca}}$ potassium channels and influx of potassium ions, leading to vascular relaxation in an endothelium-independent manner [116]. 
b) Silver Nanoparticles. Perhaps the most utilized metallic nanoparticle, silver nanoparticles (AgNP) are an almost ubiquitous component in the health industry and food storage. The strongantibacterial properties of AgNP have led to their wide application from home appliances to water treatment [12]. Silver ions released by AgNP collapse the proton motive force in Vibrio cholera, resulting in cell death [32]. Silver ions depress the activity of bacterial respiratory chain dehydrogenases and damage bacterial cell membrane structural integrity [70]. Cotton fibers containing AgNP display potent antibacterial activity against major human pathogens, including Staphylococcus aureus [70]. These antibacterial capabilities may have inadvertently fostered the notion that AgNP could affect mammalian systems in a similar manner.

AgNP also display unique optical properties that arise from the oscillations of conduction electrons at an excitation wavelength of around $385 \mathrm{~nm}$ [38]. AgNP possess enhanced absorption and scattering intensities compared to other nanoparticles. The wavelength of the emitted light is dependent on both particle size and surface refractive index. These properties as well as the inherent catalytic property of AgNP allow them to be potentially useful as nanoscale sensors and optical data storage devices [61].

Like AuNP, AgNP exposure has been associated with antiangiogenic effects, suggesting a shared mechanism of vascular toxicity by metal nanoparticles. Vascular endothelial growth factor (VEGF)-induced cell proliferation, migration as well as capillary formation in bovine retinal endothelial cells were markedly reduced following exposure via inhibition of the PI3K/Akt pathway [46]. 
In an in vitro BBB model, isolated rat brain microvascular endothelial cells treated with increasing AgNP sizes (25, 40 and $80 \mathrm{~nm}$ ) showed an increased cytotoxicity and proinflammatory response in both a size and time (4 and 8 hours) dependent manner [124]. Larger AgNP were associated with a less severe inflammatory response and microvascular cytotoxicity. The time-dependence of the cytotoxic effects of AgNP was also visible in human microvascular endothelial cells and endothelial-colony forming cells. At high concentrations, AgNP can damage cell membranes, initiate DNA damage and stimulate the formation of free radicals. Interestingly, at sublethal concentrations, microvascular cells remained viable but showed a significant impairment in proliferation [19]. In isolated aortic rings, AgNP exposure resulted in a dose-dependent inhibition of nitric oxide-mediated relaxation, while coronary vessel dilation was unimpaired [43]. AgNP were preferentially internalized by human umbilical vein endothelial cells, resulting in the formation of reactive oxygen species (ROS), cytoskeletal actin rearrangement, and disruption of intercellular endothelial integrity. Increased vascular permeability was observed in Balb/c mice exposed intravenously to AgNP leading to the systemic release of AgNP and peripheral inflammation in the liver, lungs and kidneys [44]. Endothelial cell injury, dysfunction and oxidative stress induced by exposure to AgNP occurs due to activation of the inflammatory IKK/NF-KB pathways [114].

c) Copper. Copper nanoparticles (CuNP) have also been successfully used as antimicrobial agents, in textiles, plastics and as additives for coatings. The antimicrobial activity of CuNP has been associated with ROS generation, nucleic acid damage, lipid and protein oxidation [21]. The high thermal conductivity and catalytic activity of CuNP make them efficient as sintering additives and catalysts for chemical reactions such as in the synthesis of methanol and glycol. Notwithstanding these industrial uses, CuNP are classified as a class 3 (moderately toxic) agent with cytotoxic and proinflammatory effects being reported in vitro [123]. The direct toxic effects 
of CuNP on the different components of the cardiovascular system are less well understood and additional research is warranted. Porcine brain microvessel endothelial cells treated with $15 \mu \mathrm{g} / \mathrm{ml}$ CuNP of different sizes (40 and $60 \mathrm{~nm}$ ) increased cellular permeability in a size-dependent manner with a greater cytotoxic effect visible with smaller particles. The increased permeability has been linked with increased levels of the proinflammatory cytokines TNF- $\alpha$, IL-1 $\beta$, and prostaglandins (86). CuNP have also been linked with ROS generation and inflammation in mouse pulmonary epithelial cells and human cardiac microvascular endothelial cells [121]. In a chick embryo model, CuNP exposure resulted in marked proangiogenic and proliferative effect via upregulation of VEGF and other angiogenic factors $[83,84]$.

It is noteworthy that modulation of VEGF signaling is a recurring theme in the antiangiogenic effects of metallic ENM. This effect may occur due to inhibition of phosphorylation of associated downstream effector molecules including Src [56] and Akt [97] leading to increased apoptosis of endothelial cells [40], impaired hematopoietic cell development, survival, differentiation, and migration $[66,79]$, aberrant vasculogenesis and heart development in prenatally exposed subjects [80].

\section{ii) Metal Oxides}

a) Titanium Dioxide Nanoparticles. $\mathrm{Nano}-\mathrm{TiO}_{2}$ is among the most widely used nanomaterials. Its pervasive use has caused nano- $\mathrm{TiO}_{2}$ to be associated with systemic effects and to be classified as a potential occupational carcinogen to humans [90]. Its ability to function as a high-energy electron donor following treatment with UV light makes nano- $\mathrm{TiO}_{2}$ a versatile $\mathrm{ENM}$ with

applications in consumer products such as topical sunscreens and cosmetics and industrial components including paints [115]. 
Pulmonary nano- $\mathrm{TiO}_{2}$ exposure has been associated with systemic inflammation in vivo [91, 132]. Leukocyte activation, the release of proinflammatory mediators, oxidative stress and DNA damage [27] leading to endothelium-dependent dysfunction. In isolated subepicardial arterioles of rats exposed to nano- $\mathrm{TiO}_{2}$, (primary particle diameter $\sim 21 \mathrm{~nm}$ ) a significant impairment in endothelium-dependent vasodilation was noted [67]. Similar findings were reported in other vascular beds including the uterus [117] and the spinotrapezius muscle [92]. This endotheliumdependent microvascular dysfunction has been linked with a reduced nitric oxide bioavailability triggered by the generation of ROS following exposure or via the initiation of inflammatory mechanisms in the lung $[68,93]$. Similarly, acute (24 hour) exposure to nano- $\mathrm{TiO}_{2}$ reduced microvascular nitric oxide bioavailability and altered cyclooxygenase-mediated vasoreactivity. An increased sympathetic tone was seen in exposed rats, indicating an enhanced adrenergic sensitivity via upregulation of $\alpha$-adrenergic receptor density or increased release of neurotransmitters such as norepinephrine and neuropeptide $Y$ at neurovascular junctions [58]. Thus, an augmented sympathetic tone could have a significant impact on the microcirculation, particularly capacitance vessels, which show a more pronounced response to $\alpha$-adrenergic stimulation [1]. The increase in venous tone may result in greater capillary filtration or reduced reabsorption, reduced plasma volume, and increased hematocrit.

The mechanisms underlying the effects of nano- $\mathrm{TiO}_{2}$ exposure on the vasculature are well documented. Nano- $\mathrm{TiO}_{2}$ exposure may lead to an impaired vascular function via ROS which react with nitric oxide produced by endothelial cells, decreasing its bioavailability [93]. In addition, Pulmonary exposure to nano- $\mathrm{TiO}_{2}$ causes the systemic release of proinflammatory cytokines and increased leukocyte activation. Lastly, an increase in sympathetic activation and altered cyclooxygenase signaling caused by nano- $\mathrm{TiO}_{2}$ have also been shown to affect vascular reactivity 
[60]. Moreover, inhalation of nano- $\mathrm{TiO}_{2}$ also increases sympathetic tone via activation of the transient receptor potential channel in pulmonary C-fiber sensory neurons. The increased C-fiber activity augments neuronal function in the nodose ganglion and consequently affects autonomic regulation by the medullary cardiovascular regulatory center [57].

b) Cerium dioxide Nanoparticles. The catalytic properties of $\mathrm{CeO}_{2}-\mathrm{NP}$ have made them excellent diesel fuel additive [18] to increase combustion efficiency. Also, the capability of $\mathrm{CeO}_{2}-\mathrm{NP}$ to shift between two valence states $\left(\mathrm{Ce}^{3+}\right.$ and $\left.\mathrm{Ce}^{4+}\right)$ by reacting with highly reactive free radicals such as superoxide make it useful as an antioxidant agent [26].

Systemic exposure to $\mathrm{CeO}_{2}-\mathrm{NP}$ results in a time-dependent increase in oxidative stress $[138,139]$. The long-term persistence and adverse effects associated with $\mathrm{CeO}_{2}-\mathrm{NP}$ exposure have raised significant concerns about its therapeutic potential as an antioxidant. Its impact on cardiovascular function is poorly understood but recent studies with human aortic endothelial cells exposed acutely (4 hours) to $\mathrm{CeO}_{2}-\mathrm{NP}$ at different concentrations $(0.001-50 \mu \mathrm{g} / \mathrm{ml})$ revealed a modest elevation of inflammatory markers such as intercellular adhesion molecule 1 (ICAM-1), MCP-1 and IL-8 [42]. While this study shows a modest effect on the macrocirculation, previous work conducted by our lab has shown that pulmonary exposure to $\mathrm{CeO}_{2}-\mathrm{NP}$ impairs coronary and microvascular endothelium-dependent and independent dilation in a dose- and route-dependent manner $[77,78]$. The microvascular dysfunction seen in the microcirculation was linked to ROS production, decreased nitric oxide production and an elevated level of inflammatory cytokines [77]. 
Like nano- $\mathrm{TiO}_{2}$, exposure to $\mathrm{CeO}_{2}-\mathrm{NP}$ may trigger ROS generation, via nitric oxide synthase uncoupling and mitochondrial damage, thus affecting endothelium-derived nitric oxide signaling and vascular smooth muscle function. An inflammatory response to $\mathrm{CeO}_{2}-\mathrm{NP}$ exposure has been suggested to play a role in these vascular effects [77].

c) Iron Oxide Nanoparticles. In recent years, increasing investigations with various types of iron oxide nanoparticles (FeO-NP) (mostly maghemite, $\mathrm{y}-\mathrm{Fe}_{\mathrm{e}} \mathrm{O}_{3}$, and magnetite, $\mathrm{Fe}_{\mathrm{e}} \mathrm{O}_{4}$ ) have explored the potential biomedical applications. Based on their thermal and electrical conductivity and magnetic properties, FeO-NP have found applications as targeted drug delivery systems, in magnetic resonance imaging, and as a contrasting agent [45].

Despite these clinical applications, the use of FeO-NP has come under scrutiny due to the pronounced cytotoxic effects induced by exposure. For example, occupational exposures of FeONP induce severe complications such as siderosis and hemochromatosis due to iron overload $[15,52]$. Similarly, cytotoxic effects to vascular endothelium due to the catalytic generation of ROS through Fenton chemistry FeO-NP $[16,142]$ have been reported. The generation of free radicals was measured in human umbilical endothelial cells treated with dextran stabilized FeO-NP in vitro [134]. In addition, particle phagocytosis by monocytes resulted in significant oxidative stress and severe endothelial toxicity [142]. Furthermore, intravenous injection of FeO-NP (0.4, 2 and 10 $\mu \mathrm{g} / \mathrm{kg}$ ) in mice initiated significant prothrombotic effects in pial arterioles and venules as early as 1-hour post-exposure, along with increased cardiac levels of markers of oxidative stress, including lipid peroxidation, ROS levels and increased superoxide dismutase activity [86]. 
d) Zinc Oxide Nanoparticles. ZnO-NP have been historically used as an anti-bacterial and in cosmetics such as sunscreen lotions due to their UV-blocking ability. However, severe systemic and cytotoxic effects have been linked with ZnO-NP exposure. DNA fragmentation, mitochondrial damage and toxicity through ROS generation have been reported in diverse in vitro studies $[112,121]$ and in vivo exposure models [58].

In vitro ZnO-NP exposure caused significant cytotoxic effects on human cardiac microvascular endothelial cells and elicited a strong inflammatory response associated with the dissolution of ZnO-NP and the release of highly reactive ionic groups [135]. ZnO-NP internalized by human aortic endothelial cells showed pronounced inflammation and cell death [41, 101, 125].

\section{ii) Carbonaceous Nanomaterials}

a) Single Walled Carbon Nanotubes. Single-walled carbon nanotubes (SWCNT) are ENM which can be visualized as a single graphene sheet is rolled up to form a cylinder $[28,90]$. Biomedical applications include utilization as anti-microbial agents and drug-delivery systems. Inhaled SWCNT deposits efficiently in the lungs and induces inflammatory changes including the release of thrombogenic factors, particularly plasminogen activator inhibitor 1 (PAI-1) [37]. PAl-1 is a serine protease inhibitor responsible for the inhibition of plasminogen activation, intravascular fibrinolysis, cell-associated proteolysis and blood clot dissolution [26]. Under normal conditions, PAl-1 is present in plasma in very low concentrations $(5-20 \mathrm{ng} / \mathrm{ml})$ and is secreted primarily by liver cells, smooth muscle cells, adipocytes and platelets. However, in diseased conditions such as chronic inflammation, PAI-1 is also secreted by activated leukocytes and endothelial cells upon stimulation by inflammatory cytokines (for example TGF- $\beta$ ) [75]. Increased circulating PAI-1 levels have been associated with atherosclerosis and coronary artery disease [11]. 
Effects on aortic rings have been previously noted [47], with SWCNT exposure stimulating endothelial nitric oxide-independent mechanisms of vasorelaxation. Conversely, acetylcholinetriggered vasorelaxation was unaltered in $\mathrm{ApoE}^{-/-}$mice intratracheally instilled with SWCNT and no inflammatory response was detected in isolated aortic segments [127]. Similar studies have reported contrasting evidence with increased cytotoxicity, and disruption of endothelial cell cytoskeletal integrity in vitro [128]. Several in vitro studies have also indicated antiangiogenic effects, decreased nitric oxide production and ROS generation in dermal microvascular endothelial cells [7] and umbilical vein endothelial cells [108].

b) Multi Walled Carbon Nanotubes. MWCNT consist of multiple concentric graphene sheets rolled up to form a cylinder. An ever-increasing number of MWCNT are being used as drug delivery systems, tracers, contrast agents as well scaffolding for tissue [30].

The systemic effects of MWCNT exposure raise major concerns over their widespread use. Pulmonary exposure to MWCNT has been associated with inflammatory responses like those triggered by asbestos [98]. Furthermore, MWCNT have been shown to stimulate the production of cytokines and induce cytotoxicity [48]. In the macrocirculation, human aortic endothelial cells exposed in vitro to MWCNT for 24 hours at concentrations of $1.5-4.5 \mu \mathrm{g} / \mathrm{ml}$ showed significant actin filament and cadherin disruption and cytotoxicity [128]. Pulmonary instillation of SpragueDawley rats with increasing doses $(1,10$ or $100 \mu \mathrm{g})$ of MWCNT promoted cardiac injury by increasing premature ventricular contractions and myocardial infarctions in an ischemia / reperfusion model. Higher levels of endothelin-1, thromboxane, cyclooxygenase and Rho-kinase correlated with increased coronary constriction and reduced blood flow [122]. Rho-kinase 
signaling was also increased in pregnant Sprague-Dawley rates exposed to MWCNT on gestational days 17-19, resulting in an increased contractile response in uterine artery segments, mesenteric artery but not in the umbilical vein [122].

In a time-course study performed by our research program [119], MWCNT inhalation resulted in significant pulmonary infiltration of inflammatory cells and systemic translocation of ENM 24 hours post-inhalation. Coronary arteriolar function assessed $24-168$ hours post-exposure indicated an impairment of endothelium dependent dilation within 24 hours. After 168 hours, this condition showed modest improvement but was still partially present.

\section{Human health effects of ENM}

An increasing body of research in both humans and experimental animal models has raised concerns regarding the potential biological effects of ENM in humans [111]. While these studies built from knowledge gained from other occupational and environmental exposures (e.g., ambient particulate matter, asbestos, silica), the potential long-term effects such as fibrosis and cancer have not been observed because of latency. In humans, initial cross-sectional epidemiological studies have indicated early response effects due to ENM exposure [111]. Indeed, ENM have been shown to elicit oxidative stress responses in workers involved in the production or use of ENM in Taiwan $[71,72]$. Exposure to carbon nanotubes and nanofibers was associated with inflammation, fibrosis, oxidative stress, and cardiovascular biomarkers. In that same cohort, exposure was associated with respiratory allergies and altered resting heart rate but no other clinical measures [39]. Markers of lipid, nucleic acid and protein oxidation were found to be increased in the exhaled breath condensate of workers exposed to FeO-NP [100]. On a similar note, workers exposed to carbon black and MWCNT had increased blood levels of fibrinogen and 
pro-inflammatory mediators and increased or altered sputum or exhaled breath condensate levels of oxidative stress, proinflammatory and profibrotic markers of workers exposed to MWCNT $[72,99,101]$.

Occupational exposure to titanium dioxide, evaluating workers from 1935-1985, was not associated with an increased risk of developing lung cancer and other fatal respiratory diseases including pulmonary fibrosis [22]. More recently, occupational exposures to $\mathrm{TiO}_{2}$, which has a substantial nano-sized fraction, were associated with increased levels of leukotrienes in the exhaled breath condensate [101].

Overall, initial epidemiological studies have indicated effects of exposure to ENM primarily related to inflammation and oxidative stress. To date, and primarily the result of latency, cancer and chronic pulmonary effects such as fibrosis have not been observed.

\section{ENM exposure and reproductive and developmental outcomes}

A. Introduction. In recent years, the reproductive and developmental consequences of ENM exposure have become recognized as an important part of the field of toxicology. Several studies have shown the potential of ENM to impact reproduction and fetal development [53]. The mechanisms involved are unclear but may involve inflammation [53], oxidative stress [117], endocrine disruption, mitochondrial dysfunction, epigenomic changes and impairments of the maternal-fetal circulation [121]. Indeed, maternal nano- $\mathrm{TiO}_{2}$ exposure resulted in altered expression of genes associated with brain development, cell death, response to oxidative stress, and mitochondria in the brain during the prenatal period [35]. Similarly, pulmonary nano- $\mathrm{TiO}_{2}$ 
exposure was shown to induce potentially deleterious epigenomic changes, cognitive and vascular impairments in gestationally exposed progeny [36]. Gestational nano- $\mathrm{TiO}_{2}$ exposure causes a reduction in litter and pup size as well as pup number. Exposure of pregnant Slc mice to $137 \mathrm{mg} / \mathrm{kg}$ of fullerenes on GD 10 resulted in the death of all the embryos, while $50 \mathrm{mg} / \mathrm{kg}$ induced head and tail abnormalities in $50 \%$ of the fetuses [35]. Female mice administered functionalized SWCNT at 10 or $100 \mathrm{mg} / \mathrm{kg}$ on GD 9 by oral gavage increased the incidence of fetal resorptions, gross defects and skeletal abnormalities [55]. Injection of pregnant mice with SWCNT on GD 5.5 at $0.01-30 \mathrm{mg} /$ mouse increased the incidence of miscarriage and malformations such as deformities in the abdominal wall and head and limb hypoplasia. The placentas from the malformed fetuses were small and vascular damage was observed and oxidative stress was increased in the malformed fetuses and their placentas [35]. Adverse effects on spermatogenesis, histopathological changes in the testes and decreased testosterone levels have also been reported in mice following gestational exposure to carbon black [53].

The "Barker Hypothesis" proposes that fetal development within a hostile gestational environment may predispose the offspring to future health conditions and that adverse in utero and postnatal environmental influences directly increase disease susceptibility [121]. Given the critical role the uterine microcirculation plays in the regulation and distribution of blood flow to the developing fetus, any impairment in the function of this vascular bed may affect the survival of a pregnancy. Precise regulation of blood flow, especially within an environment characterized by profound remodeling and growth, is crucial for both maternal and fetal health.

\section{$B$, The Uterine Circulation.}


i) Anatomy. In humans, the uterus is supplied bidirectionally by means of a dual arterial anastomotic system with one end (ovarian artery) deriving from the aorta, while the other end (uterine artery) originates from the internal iliac artery. The bilateral arrangement of the uterine circulation creates redundancy and maintains a constant blood supply even in cases of occlusion of a vessel. Perpendicular vessels emanate from the main uterine artery and form the arcuate arteries, surround the uterus and give rise to smaller radial arteries that penetrate the myometrium. The radial arteries then branch in to the basal and/or spiral arteries at the myoendometrial border. The basal arteries form a vascular network along the myoendometrial border, while the spiral arteries enter deeper into the endometrium and end in capillaries. These capillaries are eventually drained by venules which subsequently become veins that enter the inferior vena cava. In rodents, the main utero-ovarian vessels are typically parallel to the uterine wall within a layer of connective tissue known as the mesometrium. Branches from the uteroovarian vessels connect the arcuate loops with the uterine wall. These radial arteries may then enter the uterine or widen before entering the placenta via endovascular trophoblast invasion.

C. Maternal-fetal vasculature. Vertebrates develop a system of membranes that surround the developing fetus. Apposition or fusion of these fetal membranes with the uterine mucosa, for maternal-fetal exchange, triggers the formation of the placenta. The placenta is a transient organ with significant structural and functional species variability with one common characteristic; the presence of two (maternal and fetal) discrete circulations separated by several tissue layers throughout pregnancy known as the placental barrier. This barrier typically consists of:

1) Blastocyst wall (trophoblast), which eventually becomes the chorion after fusion with the fetal mesenchyme.

2) Allantois, which represents the embryonic urinary bladder. 
3) Yolk sac, which represents an extension of the gut.

These membranes are surrounded by the amnion which forms a membranous sac derived from the fetus. Originally, the placenta was believed to be a barrier organ responsible primarily for the maternofetal exchange of waste and nutrients. However, the placenta is fundamental in a wide gamut of processes:

1) Excretion, water balance and $\mathrm{pH}$ regulation

2) Catabolic and resorptive functions

3) Synthesis and secretion of hormones

4) Metabolic and secretory functions

5) Hematopoiesis

6) Heat and gas transfer

7) Immunological functions

In humans, the placenta is characterized by a tree-like branching pattern of the chorion, resulting in the formation of the placental villous tree. These villi fit into corresponding endometrial crypts or are directly surrounded by maternal blood. Therefore, the functional unit of the human placenta is defined as the chorionic villus, which contains the syncytiotrophoblasts/cytotrophoblasts, villous stromal, and fetal vascular endothelium that separate maternal blood from the fetal circulation. The structure of the rodent placenta is often described as "labyrinthine" and is characterized by the formation of web-like channels by the trophoblasts that are filled with maternal blood or fetal capillaries. 
The uteroplacental circulation (Figure 2) begins with the flow of maternal blood into the intervillous space via the decidual spiral arteries. It is at this point that gas and nutrient exchange occurs in the intervillous space. Maternal arterial blood forces deoxygenated blood into the endometrial and uterine veins into the maternal circulation. The feto-placental circulation consists of the umbilical cord that attaches the placenta to the fetus. The cord is not directly attached to the maternal circulation but joins the placenta which mediates the transfer of substances to and from the maternal circulation. The umbilical cord contains one vein (umbilical vein) and two arteries (umbilical arteries). The umbilical vein is responsible for carrying oxygenated, nutrient-rich blood from the placenta to the fetus, while the umbilical arteries carry deoxygenated, nutrient-depleted blood from the developing fetus to the placenta. The functional units that allow the maternal-fetal exchange of nutrients and oxygen is the terminal villi. For this reason, the flow of maternal-fetal blood in humans is referred to as multivillous.

\section{Conclusion.}

Evidence has accumulated that ENM exposure negatively and diversely affects cardiovascular health. Cardiovascular endpoints associated with pulmonary ENM exposure however do not depend solely on translocation to the systemic circulation. Dysfunction may occur via activation of the immune system, resulting in the release of inflammatory mediators in the systemic circulation or via dysregulation of the autonomic nervous system [88]. Therefore, in investigating the cardiovascular impact of ENM it is critical to bear in mind that all these systems may be at play, at differing intensities over time. The severity and location of these effects is heavily dependent upon the physicochemical properties of the ENM in question and the physiological conditions of the cardiovascular continuum. A major target of ENM exposure is the endothelium, a one cell-thick layer present in all vascular compartments and responsible for the secretion of and response to paracrine, autocrine, and endocrine substances. ENM exposure has been 
implicated with impaired endothelium-dependent dilation due to decreased nitric oxide bioavailability resulting from ROS generation or via inflammatory mechanisms.

\section{Effect of Size}

Cardiovascular toxicity is inversely correlated to particle size, most likely due to the increased ability of smaller particles to deposit and penetrate deep within the site of entry, traverse biological barriers, and access the systemic circulation. Even though systemic translocation of ENM from the site of entry may not be significant [81], intentional introduction of ENM or modification of ENM properties may increase significantly the proportion entering the circulation [94] and accumulating at a target tissue [62]. Alternatively, the high number of particles or the large surface area available for interactions after an exposure may play a role. Future studies focused on the most appropriate dose-metric for these issues are warranted.

The decreased size of ENM also means an increased reactivity, a phenomenon which becomes exceedingly important when considering metal oxides ENM and their catalytic ability to generate ROS via Fenton chemistry. Increased reactivity may also increase the likelihood for ENM to interact with biomolecules, thus increasing their potential immunogenicity and overall toxicity.

\section{Effect of Shape}

ENM shape is also a key determinant of cytotoxicity. Carbonaceous ENM, particularly SWCNT and MWCNT are classical examples of high aspect-ratio ENM. Some studies have shown that SWCNT exhibit greater immunogenicity and cytotoxicity than MWCNT because of their unique physical conformation [55]. Their systemic, vascular effects and persistence may be a result of 
this structural property. An increased aspect-ratio could prevent clearance by cells of the immune system and promote deposition within the vasculature particularly at bifurcations or in regions of turbulent flow. ENM with higher aspect-ratios have a greater tendency to settle out of centerline blood flow and deposit within the vascular wall compared to their spherical counterparts.

\section{Effect of Surface Charge and Chemistry}

Surface charge and chemistry may influence the biological effects of ENM. Clearance by the kidneys, particle agglomeration and the interaction with both immune and cardiovascular components are impacted by ENM surface properties. Charged particles elicit a more significant inflammatory response via activation of the mononuclear phagocytic system and the caspaseinflammasome axis. ENM functionalization via the addition of specific chemical moieties may greatly dictate the interaction with physiological biomolecules and therefore the formation of a protein corona. Within the vasculature, changes in ENM surface chemistry can affect ENM agglomeration which in turn may increase ENM plasma half-life and vascular deposition.

\section{Reactivity - Tissue Specificity}

The impact of ENM exposure on the systemic circulation and cardiovascular health are tissue specific. The macrocirculation function as blood conduits and pressure reservoirs. While these functions are fundamental for cardiovascular health, they do not depend on significant degrees of vasomotion and regulation by central and local mechanisms. Therefore, ENM deposition in the vascular system may exacerbate preexisting cardiovascular conditions such as atherosclerosis [2]. Damage to the macrocirculation leads to a decreased vascular compliance and consequently an increase in afterload. A prolonged increase in afterload and vascular resistance may lead to increased work by the heart and eventually cardiac failure. 
The microcirculation is a major regulatory functional component of the cardiovascular system. Its fundamental role in the generation of total peripheral resistance, immune response and waste exchange are well known. ENM deposition in arterioles may lead to impaired vascular reactivity, thus affecting the ability of these vessels to generate resistance to blood flow This impaired reactivity could have major effects on tissue perfusion and nutrient-waste exchange in capillaries. ENM deposition within capillaries may severely impact vascular permeability by damaging endothelial cells cytoskeleton. The release of ions by ENM could affect the subtle balance between capillary and interstitial fluid colloid and oncotic pressures. Localization of ENM within venules may promote leukocytic infiltration and trigger an innate immune response and the release of proinflammatory mediators. Damage to venules may increase capillary filtration and hematocrit.

In conclusion, the field of cardiovascular nanotoxicology is still in its relative infancy. With the increasing presence of ENM in everyday consumer products, the risk of unintentional occupational and domestic exposure remains very high. The use of in vitro models, different exposure conditions and ENM characteristics may partly account for the incongruity in the toxicological research. However, the advent of new and more powerful experimental tools such as high throughput screening may prove beneficial for the assessment of the vascular effects of ENM. Whatever directions future research takes, the regional heterogeneity and unique ENM properties, as well as their interactions must be directly addressed if nanotechnology is to make its greatest contribution to human health. 
While the current literature consistently points towards an impairment in overall cardiovascular function associated with ENM exposure, the cardiovascular system is comprised of various segments that are fundamentally different in their function and structure. The present studies were designed to evaluate 1) the effect of ENM exposure on different levels of the vasculature to identify the most sensitive discrete vascular components to ENM toxicity, and 2) identify potential mechanisms involved in the microvascular and reproductive effects of pulmonary exposure. The findings of these studies should further contribute to our knowledge of the interaction among the different microvascular control mechanisms and how ENM exposure alters these mechanisms.

\section{Purpose of Dissertation}

Given the almost ubiquitous inclusion of ENM in everyday products, identification of the biological effects of these materials is important if the nanotechnology industry is to reach its full potential. Nano- $\mathrm{TiO}_{2}$ is by far the most widely used ENM [89] Therefore, the present studies were designed to study: 1) the differential effects of nano- $\mathrm{TiO}_{2}$ on discrete segments of the vasculature and identify the most sensitive segments to pulmonary ENM exposure, 2) the acute microvascular and immunological effects of pulmonary nano- $\mathrm{TiO}_{2}$ exposure and provide a mechanistic basis for the observed outcomes, 3 ) the effects of gestational pulmonary nano- $\mathrm{TiO}_{2}$ exposure on placental hemodynamics and the potential fetal repercussions.

The specific aims of the studies are listed below.

\section{Study 1}

The aim of this study was to identify the most sensitive segments of the vasculature to pulmonary ENM exposure. Sprague-Dawley rats were intratracheally instilled with $20 \mu \mathrm{g}, 100 \mu \mathrm{g}$ or $200 \mu \mathrm{g}$ nano- $\mathrm{TiO}_{2} .24$ hours post-exposure vascular reactivity of the thoracic aorta, common femoral artery, $3^{\text {rd }}$ order mesenteric arterioles was determined by measurement of isometric tension 
generation using wire myography in vitro. Furthermore, vascular reactivity of $4^{\text {th }}$ and $5^{\text {th }}$ order mesenteric arterioles was assessed using the isolated microvessel technique. These preparations provided an understanding of the vascular mechanisms (e.g. endotheliumdependent, endothelium -independent and adrenergic) influenced by nano- $\mathrm{TiO}_{2}$ exposure. Furthermore, this study identifies the most sensitive vascular levels to pulmonary exposure.

\section{Study 2}

The aims of this second study was to identify the effect of pulmonary ENM exposure on innate lymphoid cell function and the effect on uterine arteriolar function. Virgin, female, Sprague-Dawley rats were intratracheally instilled with $100 \mu \mathrm{g}$ nano- $\mathrm{TiO}_{2}$. Serial blood samples were obtained at 0, 1, 2 and 4 hours post-exposure for cytokine analysis. Lung Group 2 innate lymphoid cell number and interleukin 33 levels in bronchoalveolar lavage fluid and serum were determined 4 hours after exposure. 24 hours post-exposure uterine radial arterioles were assessed using the isolated microvessel technique. This preparation provided an understanding of the arteriolar alterations (e.g. endothelium-dependent, endothelium-independent and adrenergic) that follow nano- $\mathrm{TiO}_{2}$ exposure. This study identifies the acute inflammatory time-course associated with pulmonary ENM exposure, provides a potential mechanism linking the observed microvascular outcomes with the pulmonary effects and proposes a role for Group 2 innate lymphoid cells.

\section{Study 3}

Lastly, the aim of this study was to determine the effects of gestational ENM exposure on placental vascular resistance and umbilical vascular function. Pregnant Sprague-Dawley rats were exposed via whole-body inhalation to nano- $\mathrm{TiO}_{2}$ on GD 11 for 7 days (6h/exposure) for a cumulative dose of $525 \pm 16 \mu \mathrm{g} .24$ post-exposure, placentas, umbilical artery and vein were assessed using the isolated microvessel preparation. This preparation provided an understanding of the arteriolar and 
placental alterations (e.g. endothelium-dependent, endothelium-independent) that follow gestational nano- $\mathrm{TiO}_{2}$ exposure. Overall, this study provided information regarding the influence of nano- $\mathrm{TiO}_{2}$ on the function of the maternal-fetal microcirculation. 


\section{Reference List:}

[1] Abboud FM. The sympathetic nervous system and alpha-adrenergic blocking agents in shock. Med.Clin.North Am. 1968;52(5)1049-1060.

[2] Abdelhalim MA, Jarrar BM. Gold nanoparticles administration induced prominent inflammatory, central vein intima disruption, fatty change and Kupffer cells hyperplasia. Lipids Health Dis. 2011;10133.

[3] Alkilany AM, Shatanawi A, Kurtz T, et al. Toxicity and cellular uptake of gold nanorods in vascular endothelium and smooth muscles of isolated rat blood vessel: importance of surface modification. Small 2012;8(8)1270-1278.

[4] Alkilany AM, Thompson LB, Boulos SP, et al. Gold nanorods: their potential for photothermal therapeutics and drug delivery, tempered by the complexity of their biological interactions. Adv.Drug Deliv.Rev. 2012;64(2)190-199.

[5] Allen TM, Chonn A. Large unilamellar liposomes with low uptake into the reticuloendothelial system. FEBS Lett. 1987;223(1)42-46.

[6] Baisch, B.L., et al., Equivalent titanium dioxide nanoparticle deposition by intratracheal instillation and whole-body inhalation: the effect of dose rate on acute respiratory tract inflammation. Part Fibre Toxicol, 2014. 11: p. 5.

[7] Bayat N, Lopes VR, Scholermann J, et al. Vascular toxicity of ultra-small TiO2 nanoparticles and single walled carbon nanotubes in vitro and in vivo. Biomaterials 2015;631-13.

[8] Berciaud S, Cognet L, Lounis B. Photothermal absorption spectroscopy of individual semiconductor nanocrystals. Nano.Lett. 2005;5(11)2160-2163.

[9] Berne RM, Koeppen.B.M., Stanton BA. Berne \& Levy Physiology, Philadelphia, Mosby/Elsevier, 2010. 
[10] Bertrand N, Leroux JC. The journey of a drug-carrier in the body: an anatomophysiological perspective. J.Control Release 2012;161(2)152-163.

[11] Binder BR, Christ G, Gruber F, et al. Plasminogen activator inhibitor 1: physiological and pathophysiological roles. News Physiol Sci. 2002;1756-61.

[12] Bosetti M, Masse A, Tobin E, et al. Silver coated materials for external fixation devices: in vitro biocompatibility and genotoxicity. Biomaterials 2002;23(3)887-892.

[13] Brenner BM, Hostetter TH, Humes HD. Glomerular permselectivity: barrier function based on discrimination of molecular size and charge. Am.J.Physiol 1978;234(6)F455-F460.

[14] Brenner BM, Hostetter TH, Humes HD. Molecular basis of proteinuria of glomerular origin. N.Engl.J.Med. 1978;298(15)826-833.

[15] Brittenham GM. New advances in iron metabolism, iron deficiency, and iron overload. Curr.Opin.Hematol. 1994;1(2)101-106.

[16] Buyukhatipoglu K, Clyne AM. Superparamagnetic iron oxide nanoparticles change endothelial cell morphology and mechanics via reactive oxygen species formation. J.Biomed.Mater.Res.A 2011;96(1)186-195.

[17] Bystrzejewska-Piotrowska G, Golimowski J, Urban PL. Nanoparticles: their potential toxicity, waste and environmental management. Waste Manag. 2009;29(9)2587-2595.

[18] Cassee FR, van Balen EC, Singh C, et al. Exposure, health and ecological effects review of engineered nanoscale cerium and cerium oxide associated with its use as a fuel additive. Crit Rev.Toxicol. 2011;41(3)213-229.

[19] Castiglioni S, Caspani C, Cazzaniga A, et al. Short- and long-term effects of silver nanoparticles on human microvascular endothelial cells. World J.Biol.Chem. 2014;5(4)457464.

[20] Champion JA, Walker A, Mitragotri S. Role of particle size in phagocytosis of polymeric microspheres. Pharm. Res. 2008;25(8)1815-1821. 
[21] Chatterjee AK, Chakraborty R, Basu T. Mechanism of antibacterial activity of copper nanoparticles. Nanotechnology. 2014;25(13)135101.

[22] Chen JL, E. F. Epidemiologic study of workers exposed to titanium dioxide. Occup Med (Chic III). 1988.

[23] Chithrani DB, Dunne M, Stewart J, et al. Cellular uptake and transport of gold nanoparticles incorporated in a liposomal carrier. Nanomedicine. 2010;6(1)161-169.

[24] Chithrani DB. Intracellular uptake, transport, and processing of gold nanostructures. Mol.Membr.Biol. 2010;27(7)299-311.

[25] Chonn A, Cullis PR, Devine DV. The role of surface charge in the activation of the classical and alternative pathways of complement by liposomes. J.Immunol. 1991;146(12)4234-4241.

[26] Christ G, Hufnagl P, Kaun C, et al. Antifibrinolytic properties of the vascular wall. Dependence on the history of smooth muscle cell doublings in vitro and in vivo. Arterioscler.Thromb.Vasc.Biol. 1997;17(4)723-730.

[27] Colon J, Herrera L, Smith J, et al. Protection from radiation-induced pneumonitis using cerium oxide nanoparticles. Nanomedicine. 2009;5(2)225-231.

[28] Cui Y, Liu H, Ze Y, et al. Gene expression in liver injury caused by long-term exposure to titanium dioxide nanoparticles in mice. Toxicol.Sci. 2012;128(1)171-185.

[29] Dai H. Carbon nanotubes: synthesis, integration, and properties. Acc.Chem.Res. 2002;35(12)1035-1044.

[30] De Jong WH, Hagens WI, Krystek P, et al. Particle size-dependent organ distribution of gold nanoparticles after intravenous administration. Biomaterials 2008;29(12)1912-1919.

[31] Deshmukh M, Kutscher HL, Gao D, et al. Biodistribution and renal clearance of biocompatible lung targeted poly(ethylene glycol) (PEG) nanogel aggregates. J.Control Release 2012;164(1)65-73.

[32] Dibrov P, Dzioba J, Gosink KK, et al. Chemiosmotic mechanism of antimicrobial activity of $\mathrm{Ag}(+)$ in Vibrio cholerae. Antimicrob.Agents Chemother. 2002;46(8)2668-2670. 
[33] Dobrovolskaia MA, Patri AK, Zheng J, et al. Interaction of colloidal gold nanoparticles with human blood: effects on particle size and analysis of plasma protein binding profiles. Nanomedicine. 2009;5(2)106-117.

[34] Dougherty GM, Rose KA, Tok JB, et al. The zeta potential of surface-functionalized metallic nanorod particles in aqueous solution. Electrophoresis 2008;29(5)1131-1139.

[35] Ema, M., et al., Reproductive and developmental toxicity of carbon-based nanomaterials: A literature review. Nanotoxicology, 2016. 10(4): p. 391-412.

[36] Engler-Chiurazzi, E.B., et al., Impacts of prenatal nanomaterial exposure on male adult Sprague-Dawley rat behavior and cognition. J Toxicol Environ Health A, 2016. 79(11): p. 44752.

[37] Erdely A, Hulderman T, Salmen R, et al. Cross-talk between lung and systemic circulation during carbon nanotube respiratory exposure. Potential biomarkers. Nano.Lett. 2009;9(1)3643.

[38] Evanoff DD, Jr., Chumanov G. Synthesis and optical properties of silver nanoparticles and arrays. Chemphyschem. 2005;6(7)1221-1231.

[39] Fatkhutdinova LM, Khaliullin TO, Vasil'yeva OL, et al. Fibrosis biomarkers in workers exposed to MWCNTs. Toxicol Appl Pharmacol. 2016. doi:10.1016/j.taap.2016.02.016

[40] Gerber HP, Hillan KJ, Ryan AM, et al. VEGF is required for growth and survival in neonatal mice. Development 1999;126(6)1149-1159.

[41] Gojova A, Guo B, Kota RS, et al. Induction of inflammation in vascular endothelial cells by metal oxide nanoparticles: effect of particle composition. Environ. Health Perspect. 2007;115(3)403-409.

[42] Gojova A, Lee JT, Jung HS, et al. Effect of cerium oxide nanoparticles on inflammation in vascular endothelial cells. Inhal.Toxicol. 2009;21 Suppl 1123-130.

[43] Gonzalez C, Rosas-Hernandez H, Ramirez-Lee MA, et al. Role of silver nanoparticles (AgNPs) on the cardiovascular system. Arch.Toxicol. 2016;90(3)493-511. 
[44] Guo H, Zhang J, Boudreau M, et al. Intravenous administration of silver nanoparticles causes organ toxicity through intracellular ROS-related loss of inter-endothelial junction. Part Fibre.Toxicol. 2016;1321.

[45] Gupta AK, Curtis AS. Lactoferrin and ceruloplasmin derivatized superparamagnetic iron oxide nanoparticles for targeting cell surface receptors. Biomaterials 2004;25(15)3029-3040.

[46] Gurunathan S, Lee KJ, Kalishwaralal K, et al. Antiangiogenic properties of silver nanoparticles. Biomaterials 2009;30(31)6341-6350.

[47] Gutierrez-Hernandez JM, Ramirez-Lee MA, Rosas-Hernandez H, et al. Single-walled carbon nanotubes (SWCNTs) induce vasodilation in isolated rat aortic rings. Toxicol.In Vitro 2015;29(4)657-662.

[48] Hirano S, Fujitani Y, Furuyama A, et al. Uptake and cytotoxic effects of multi-walled carbon nanotubes in human bronchial epithelial cells. Toxicol.Appl.Pharmacol. 2010;249(1)815.

[49] Hirn S, Semmler-Behnke M, Schleh C, et al. Particle size-dependent and surface chargedependent biodistribution of gold nanoparticles after intravenous administration. Eur.J.Pharm.Biopharm. 2011;77(3)407-416.

[50] Hirn S, Semmler-Behnke M, Schleh C, et al. Particle size-dependent and surface chargedependent biodistribution of gold nanoparticles after intravenous administration. Eur.J.Pharm.Biopharm. 2011;77(3)407-416.

[51] Honary S, Ebrahimi P, Hadianamrei R. Optimization of size and encapsulation efficiency of 5-FU loaded chitosan nanoparticles by response surface methodology. Curr.Drug Deliv. $2013 ; 10(6) 742-752$.

[52] Horwitz LD, Rosenthal EA. Iron-mediated cardiovascular injury. Vasc.Med. 1999;4(2)9399.

[53] Hougaard KS, Jackson P, Jensen KA, et al. Effects of prenatal exposure to surfacecoated nanosized titanium dioxide (UV-Titan). A study in mice. Part Fibre.Toxicol. 2010;716. 
[54] Illum SL, Davis SS. Effect of the nonionic surfactant poloxamer 338 on the fate and deposition of polystyrene microspheres following intravenous administration. J.Pharm.Sci. 1983;72(9)1086-1089.

[55] Jia G, Wang H, Yan L, et al. Cytotoxicity of carbon nanomaterials: single-wall nanotube, multi-wall nanotube, and fullerene. Environ.Sci.Technol. 2005;39(5)1378-1383.

[56] Kalishwaralal K, Sheikpranbabu S, BarathManiKanth S, et al. Gold nanoparticles inhibit vascular endothelial growth factor-induced angiogenesis and vascular permeability via Src dependent pathway in retinal endothelial cells. Angiogenesis. 2011;14(1)29-45.

[57] Kan H, Wu Z, Lin YC, et al. The role of nodose ganglia in the regulation of cardiovascular function following pulmonary exposure to ultrafine titanium dioxide. Nanotoxicology. 2014;8(4)447-454.

[58] Kim YH, Fazlollahi F, Kennedy IM, et al. Alveolar epithelial cell injury due to zinc oxide nanoparticle exposure. Am.J.Respir.Crit Care Med. 2010;182(11)1398-1409.

[59] Klibanov AL, Maruyama K, Beckerleg AM, et al. Activity of amphipathic poly(ethylene glycol) 5000 to prolong the circulation time of liposomes depends on the liposome size and is unfavorable for immunoliposome binding to target. Biochim.Biophys.Acta 1991;1062(2)142148.

[60] Knuckles TL, Yi J, Frazer DG, et al. Nanoparticle inhalation alters systemic arteriolar vasoreactivity through sympathetic and cyclooxygenase-mediated pathways. Nanotoxicology. 2012;6(7)724-735.

[61] Kossyrev PA, Yin A, Cloutier SG, et al. Electric field tuning of plasmonic response of nanodot array in liquid crystal matrix. Nano.Lett. 2005;5(10)1978-1981.

[62] Kreyling WG, Hirn S, Moller W, et al. Air-blood barrier translocation of tracheally instilled gold nanoparticles inversely depends on particle size. ACS Nano. 2014;8(1)222-233.

[63] Kreyling WG, Semmler-Behnke M, Moller W. Ultrafine particle-lung interactions: does size matter? J.Aerosol Med. 2006;19(1)74-83. 
[64] Kreyling WG, Semmler-Behnke M, Seitz J, et al. Size dependence of the translocation of inhaled iridium and carbon nanoparticle aggregates from the lung of rats to the blood and secondary target organs. Inhal.Toxicol. 2009;21 Suppl 155-60.

[65] Kreyling, W.G., et al., Air-blood barrier translocation of tracheally instilled gold nanoparticles inversely depends on particle size. ACS Nano, 2014. 8(1): p. 222-233.

[66] Kubo H, Alitalo K. The bloody fate of endothelial stem cells. Genes Dev. 2003;17(3)322329.

[67] LeBlanc AJ, Cumpston JL, Chen BT, et al. Nanoparticle inhalation impairs endotheliumdependent vasodilation in subepicardial arterioles. J.Toxicol.Environ. Health A 2009;72(24)1576-1584.

[68] LeBlanc AJ, Moseley AM, Chen BT, et al. Nanoparticle inhalation impairs coronary microvascular reactivity via a local reactive oxygen species-dependent mechanism. Cardiovasc.Toxicol. 2010;10(1)27-36.

[69] Li R, Wang X, Ji Z, et al. Surface charge and cellular processing of covalently functionalized multiwall carbon nanotubes determine pulmonary toxicity. ACS Nano. 2013;7(3)2352-2368.

[70] Li WR, Xie XB, Shi QS, et al. Antibacterial effect of silver nanoparticles on Staphylococcus aureus. Biometals 2011;24(1)135-141.

[71] Liao HY, Chung YT, Lai $\mathrm{CH}$, et al. Six-month follow-up study of health markers of nanomaterials among workers handling engineered nanomaterials. Nanotoxicology. 2014. doi:10.1002/bate.201500115

[72] Liou SH, Tsai CSJ, Pelclova D, Schubauer-Berigan MK, Schulte PA. Assessing the first wave of epidemiological studies of nanomaterial workers. J Nanoparticle Res. 2015. doi:10.1007/s11051-015-3219-7 
[73] Liu HH, Surawanvijit S, Rallo R, et al. Analysis of nanoparticle agglomeration in aqueous suspensions via constant-number Monte Carlo simulation. Environ.Sci.Technol. 2011;45(21)9284-9292.

[74] Lundqvist M, Stigler J, Elia G, et al. Nanoparticle size and surface properties determine the protein corona with possible implications for biological impacts. Proc.Natl.Acad.Sci.U.S.A 2008;105(38)14265-14270.

[75] Macfelda K, Weiss TW, Kaun C, et al. Plasminogen activator inhibitor 1 expression is regulated by the inflammatory mediators interleukin-1alpha, tumor necrosis factor-alpha, transforming growth factor-beta and oncostatin $\mathrm{M}$ in human cardiac myocytes. J.Mol.Cell Cardiol. 2002;34(12)1681-1691.

[76] Mauter MS, Elimelech M. Environmental applications of carbon-based nanomaterials. Environ.Sci.Technol. 2008;42(16)5843-5859.

[77] Minarchick VC, Stapleton PA, Fix NR, et al. Intravenous and gastric cerium dioxide nanoparticle exposure disrupts microvascular smooth muscle signaling. Toxicol.Sci. 2015;144(1)77-89.

[78] Minarchick VC, Stapleton PA, Porter DW, et al. Pulmonary cerium dioxide nanoparticle exposure differentially impairs coronary and mesenteric arteriolar reactivity. Cardiovasc.Toxicol. 2013;13(4)323-337.

[79] Miquerol L, Langille BL, Nagy A. Embryonic development is disrupted by modest increases in vascular endothelial growth factor gene expression. Development 2000;127(18)3941-3946.

[80] Mody VV, Siwale R, Singh A, et al. Introduction to metallic nanoparticles. J.Pharm.Bioallied.Sci. 2010;2(4)282-289.

[81] Moller W, Felten K, Sommerer K, et al. Deposition, retention, and translocation of ultrafine particles from the central airways and lung periphery. Am.J.Respir.Crit Care Med. 2008;177(4)426-432. 
[82] Moore A, Marecos E, Bogdanov A, Jr., et al. Tumoral distribution of long-circulating dextran-coated iron oxide nanoparticles in a rodent model. Radiology 2000;214(2)568-574.

[83] Mroczek-Sosnowska N, Lukasiewicz M, Wnuk A, et al. In ovo administration of copper nanoparticles and copper sulfate positively influences chicken performance. J.Sci.Food Agric. 2015.

[84] Mroczek-Sosnowska N, Sawosz E, Vadalasetty KP, et al. Nanoparticles of copper stimulate angiogenesis at systemic and molecular level. Int.J.Mol.Sci. 2015;16(3)4838-4849.

[85] National Nanotechnology Coordination Office. National Science and Technology Council Committee on Technology Subcommittee on Nanoscale Science Engineering and Technology; National Science and Technology Council, editor. The National Nanotechnology Initiative, Supplement to the President's FY2012 Budget. 1-50. 2012. Ref Type: Report

[86] Nemmar A, Beegam S, Yuvaraju P, et al. Ultrasmall superparamagnetic iron oxide nanoparticles acutely promote thrombosis and cardiac oxidative stress and DNA damage in mice. Part Fibre.Toxicol. 2016;13(1)22.

[87] Nemmar A, Hoet PH, Vanquickenborne B, et al. Passage of inhaled particles into the blood circulation in humans. Circulation 2002;105(4)411-414.

[88] Newby DE, Mannucci PM, Tell GS, et al. Expert position paper on air pollution and cardiovascular disease. Eur.Heart J. 2015;36(2)83-93b.

[89] NIOSH, DHHS. Current Intelligence Bulletin 63 - Occupational Exposure to Titanium Dioxide. 2011. Ref Type: Report

[90] Niyogi S, Hamon MA, Hu H, et al. Chemistry of single-walled carbon nanotubes. Acc.Chem.Res. 2002;35(12)1105-1113.

[91] Nurkiewicz TR, Porter DW, Barger M, et al. Systemic microvascular dysfunction and inflammation after pulmonary particulate matter exposure. Environ.Health Perspect. $2006 ; 114(3) 412-419$. 
[92] Nurkiewicz TR, Porter DW, Hubbs AF, et al. Nanoparticle inhalation augments particledependent systemic microvascular dysfunction. Part Fibre.Toxicol. 2008;51.

[93] Nurkiewicz TR, Porter DW, Hubbs AF, et al. Pulmonary nanoparticle exposure disrupts systemic microvascular nitric oxide signaling. Toxicol.Sci. 2009;110(1)191-203.

[94] Oberdorster G, Maynard A, Donaldson K, et al. Principles for characterizing the potential human health effects from exposure to nanomaterials: elements of a screening strategy. Part Fibre.Toxicol. 2005;28.

[95] Osmond-McLeod MJ, Poland CA, Murphy F, et al. Durability and inflammogenic impact of carbon nanotubes compared with asbestos fibres. Part Fibre.Toxicol. 2011;815.

[96] Palombo M, Deshmukh M, Myers D, et al. Pharmaceutical and toxicological properties of engineered nanomaterials for drug delivery. Annu.Rev.Pharmacol.Toxicol. 2014;54581-598.

[97] Pan Y, Wu Q, Qin L, et al. Gold nanoparticles inhibit VEGF165-induced migration and tube formation of endothelial cells via the Akt pathway. Biomed.Res.Int. 2014;2014418624.

[98] Paszek E, Czyz J, Woznicka O, et al. Zinc oxide nanoparticles impair the integrity of human umbilical vein endothelial cell monolayer in vitro. J.Biomed.Nanotechnol. 2012;8(6)957-967.

[99] Pelclova D, Zdimal V, Fenclova Z, et al. Markers of oxidative damage of nucleic acids and proteins among workers exposed to TiO2 (nano) particles. Occup Environ Med. 2015. doi:10.1136/oemed-2015-103161

[100] Pelclova D, Zdimal V, Kacer P, et al. Markers of nucleic acids and proteins oxidation among office workers exposed to air pollutants including (nano)TiO2particles. In: Neuroendocrinology Letters. ; 2016. doi:10.1515/reveh-2016-0030

[101] Pelclova D, Zdimal V, Kacer P, et al. Markers of lipid oxidative damage among office workers exposed intermittently to air pollutants including nanoTiO2 particles. Rev Environ Health. 2017. doi:10.1515/reveh-2016-0030 
[102] Podila R, Chen R, Ke PC, et al. Effects of surface functional groups on the formation of nanoparticle-protein corona. Appl.Phys.Lett. 2012;101(26)263701.

[103] Popel AS, Johnson PC. Microcirculation and Hemorheology. Annu.Rev.Fluid Mech. 2005;3743-69.

[104] Pries AR, Secomb TW, Gaehtgens P. Biophysical aspects of blood flow in the microvasculature. Cardiovasc.Res. 1996;32(4)654-667.

[105] Pries AR, Secomb TW. Rheology of the microcirculation. Clin.Hemorheol.Microcirc. 2003;29(3-4)143-148.

[106] Rajagopalan S, Brook RD. The Indoor-Outdoor Air-Pollution Continuum and the Burden of Cardiovascular Disease: An Opportunity For Improving Global Health. Glob.Heart 2012;7(3)207-213.

[107] Rittermeier A, Miao S, Schroter MK, et al. The formation of colloidal copper nanoparticles stabilized by zinc stearate: one-pot single-step synthesis and characterization of the coreshell particles. Phys.Chem.Chem.Phys. 2009;11(37)8358-8366.

[108] Rodriguez-Yanez Y, Bahena-Uribe D, Chavez-Munguia B, et al. Commercial singlewalled carbon nanotubes effects in fibrinolysis of human umbilical vein endothelial cells. Toxicol.In Vitro 2015;29(5)1201-1214.

[109] Sato Y, Yokoyama A, Shibata K, et al. Influence of length on cytotoxicity of multi-walled carbon nanotubes against human acute monocytic leukemia cell line THP-1 in vitro and subcutaneous tissue of rats in vivo. Mol.Biosyst. 2005;1(2)176-182.

[110] Schatteman GC, Awad O. Hemangioblasts, angioblasts, and adult endothelial cell progenitors. Anat.Rec.A Discov.Mol.Cell Evol.Biol. 2004;276(1)13-21.

[111] Schulte P, Leso V, Niang M, lavicoli I. Biological monitoring of workers exposed to engineered nanomaterials. Toxicology Letters. 2018. 
[112] Shannahan JH, Podila R, Aldossari AA, et al. Formation of a protein corona on silver nanoparticles mediates cellular toxicity via scavenger receptors. Toxicol.Sci. 2015;143(1)136146.

[113] Sharma J, Tai Y, Imae T. Biomodulation approach for gold nanoparticles: synthesis of anisotropic to luminescent particles. Chem.Asian J. 2010;5(1)70-73.

[114] Sharma V, Singh P, Pandey AK, et al. Induction of oxidative stress, DNA damage and apoptosis in mouse liver after sub-acute oral exposure to zinc oxide nanoparticles. Mutat.Res. 2012;745(1-2)84-91.

[115] Shi J, Sun $X$, Lin $Y$, et al. Endothelial cell injury and dysfunction induced by silver nanoparticles through oxidative stress via IKK/NF-kappaB pathways. Biomaterials 2014;35(24)6657-6666.

[116] Silva RM, Teesy C, Franzi L, et al. Biological response to nano-scale titanium dioxide (TiO2): role of particle dose, shape, and retention. J.Toxicol.Environ.Health A 2013;76(16)953-972.

[117] Soloviev A, Zholos A, Ivanova I, et al. Plasmonic gold nanoparticles possess the ability to open potassium channels in rat thoracic aorta smooth muscles in a remote control manner. Vascul.Pharmacol. 2015;72190-196.

[118] Stapleton PA, McBride CR, Yi J, et al. Uterine microvascular sensitivity to nanomaterial inhalation: An in vivo assessment. Toxicol.Appl.Pharmacol. 2015;288(3)420-428.

[119] Stapleton PA, Minarchick VC, Cumpston AM, et al. Impairment of coronary arteriolar endothelium-dependent dilation after multi-walled carbon nanotube inhalation: a time-course study. Int.J.Mol.Sci. 2012;13(11)13781-13803.

[120] Stapleton PA, Nurkiewicz TR. Vascular distribution of nanomaterials. Wiley.Interdiscip.Rev.Nanomed.Nanobiotechnol. 2014;6(4)338-348. 
[121] Stapleton, P.A., et al., Maternal engineered nanomaterial exposure and fetal microvascular function: does the Barker hypothesis apply? Am. J. Obstet. Gynecol, 2013. 209(3): p. 227-11.

[122] Sun J, Wang S, Zhao D, et al. Cytotoxicity, permeability, and inflammation of metal oxide nanoparticles in human cardiac microvascular endothelial cells: cytotoxicity, permeability, and inflammation of metal oxide nanoparticles. Cell Biol.Toxicol. 2011;27(5)333-342.

[123] Thompson LC, Urankar RN, Holland NA, et al. C(6)(0) exposure augments cardiac ischemia/reperfusion injury and coronary artery contraction in Sprague Dawley rats. Toxicol.Sci. 2014;138(2)365-378.

[124] Trickler WJ, Lantz SM, Murdock RC, et al. Silver nanoparticle induced blood-brain barrier inflammation and increased permeability in primary rat brain microvessel endothelial cells. Toxicol.Sci. 2010;118(1)160-170.

[125] Trickler WJ, Lantz-McPeak SM, Robinson BL, et al. Porcine brain microvessel endothelial cells show pro-inflammatory response to the size and composition of metallic nanoparticles. Drug Metab Rev. 2014;46(2)224-231.

[126] Tsou TC, Yeh SC, Tsai FY, et al. Zinc oxide particles induce inflammatory responses in vascular endothelial cells via NF-kappaB signaling. J.Hazard.Mater. 2010;183(1-3)182-188.

[127] Tsuda A, Henry FS, Butler JP. Particle transport and deposition: basic physics of particle kinetics. Compr.Physiol 2013;3(4)1437-1471.

[128] Vesterdal LK, Folkmann JK, Jacobsen NR, et al. Pulmonary exposure to carbon black nanoparticles and vascular effects. Part Fibre.Toxicol. 2010;733.

[129] Walker VG, Li Z, Hulderman T, et al. Potential in vitro effects of carbon nanotubes on human aortic endothelial cells. Toxicol.Appl.Pharmacol. 2009;236(3)319-328.

[130] Walkey CD, Olsen JB, Guo H, et al. Nanoparticle size and surface chemistry determine serum protein adsorption and macrophage uptake. J.Am.Chem.Soc. 2012;134(4)2139-2147. 
[131] Wang J, Byrne JD, Napier ME, et al. More effective nanomedicines through particle design. Small 2011;7(14)1919-1931.

[132] Wang $\mathrm{Y}$, Guo S, Chen $\mathrm{H}$, et al. Facile fabrication of large area of aggregated gold nanorods film for efficient surface-enhanced Raman scattering. J.Colloid Interface Sci. 2008;318(1)82-87.

[133] Warheit DB, Webb TR, Reed KL, et al. Pulmonary toxicity study in rats with three forms of ultrafine-TiO2 particles: differential responses related to surface properties. Toxicology 2007;230(1)90-104.

[134] Wong, B.A., Inhalation exposure systems: design, methods and operation. Toxicol Pathol, 2007. 35(1): p. 3-14.

[135] Wu X, Tan Y, Mao H, et al. Toxic effects of iron oxide nanoparticles on human umbilical vein endothelial cells. Int.J.Nanomedicine. 2010;5385-399.

[136] Xia T, Kovochich M, Liong M, et al. Comparison of the mechanism of toxicity of zinc oxide and cerium oxide nanoparticles based on dissolution and oxidative stress properties. ACS Nano. 2008;2(10)2121-2134.

[137] Xia T, Kovochich M, Liong M, et al. Polyethyleneimine coating enhances the cellular uptake of mesoporous silica nanoparticles and allows safe delivery of siRNA and DNA constructs. ACS Nano. 2009;3(10)3273-3286.

[138] Yoffe AD. Low-Dimensional Systems - Quantum-Size Effects and Electronic-Properties of Semiconductor Microcrystallites (Zero-Dimensional Systems) and Some Quasi-2Dimensional Systems. Advances in Physics 1993;42(2)173-266.

[139] Yokel RA, Au TC, MacPhail R, et al. Distribution, elimination, and biopersistence to 90 days of a systemically introduced $30 \mathrm{~nm}$ ceria-engineered nanomaterial in rats. Toxicol.Sci. 2012;127(1)256-268.

[140] Yokel RA, Tseng MT, Dan M, et al. Biodistribution and biopersistence of ceria engineered nanomaterials: size dependence. Nanomedicine. 2013;9(3)398-407. 
[141] Zhang $H$, Cui $H$. Synthesis and characterization of functionalized ionic liquid-stabilized metal (gold and platinum) nanoparticles and metal nanoparticle/carbon nanotube hybrids. Langmuir 2009;25(5)2604-2612.

[142] Zhu M, Nie G, Meng H, et al. Physicochemical properties determine nanomaterial cellular uptake, transport, and fate. Acc.Chem.Res. 2013;46(3)622-631.

[143] Zhu MT, Wang Y, Feng WY, et al. Oxidative stress and apoptosis induced by iron oxide nanoparticles in cultured human umbilical endothelial cells. J.Nanosci.Nanotechnol. 2010;10(12)8584-8590. 
Figure 1. Schematic representation of the cardiopulmonary system and identification of locations for ENM influences/activities. Black arrows: blood flow direction. Red represents oxygenated blood. Blue represents deoxygenated blood. 1) Extrapulmonary translocation to systemic circulation. 2) Arterial bifurcation and impaction. 3) Eddy current turbulence and impaction. 4) Segre-Silberg effect and plasma settling. 5) Systemic capillary translocation and tissue deposition. 6) Venular inflammatory signaling. 7) Venous settling. For simplicity, only one systemic tissue is depicted.

Figure 2. Schematic representation of the maternal-fetal circulation in rodents. Diagram showing the maternal-fetal arterial (red) and venous circulation (blue) and the direction of blood flow and the location of the trophoblast layer in rodents. 
Figures:

Figure 1:

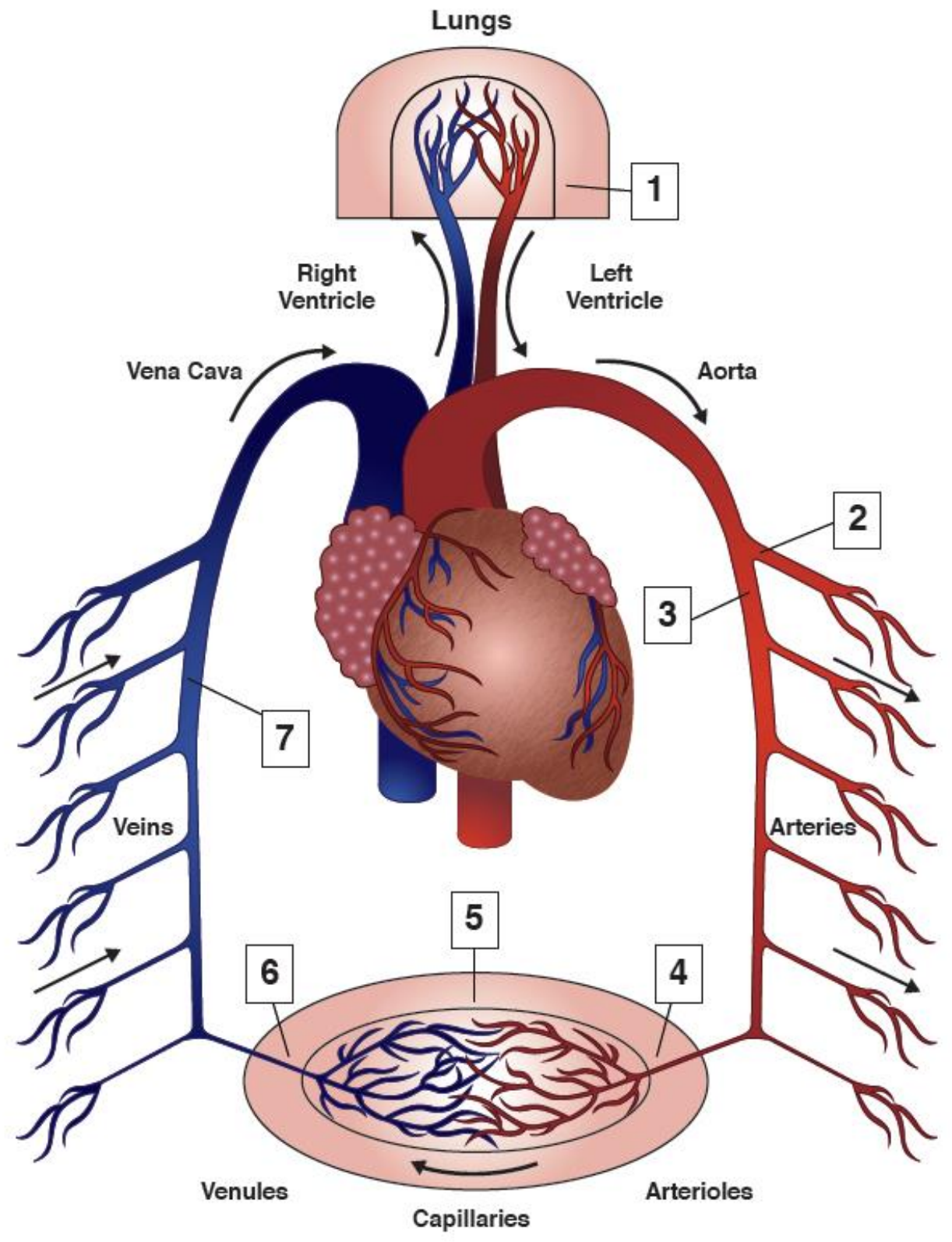

Systemic Tissues 
Figure 2:

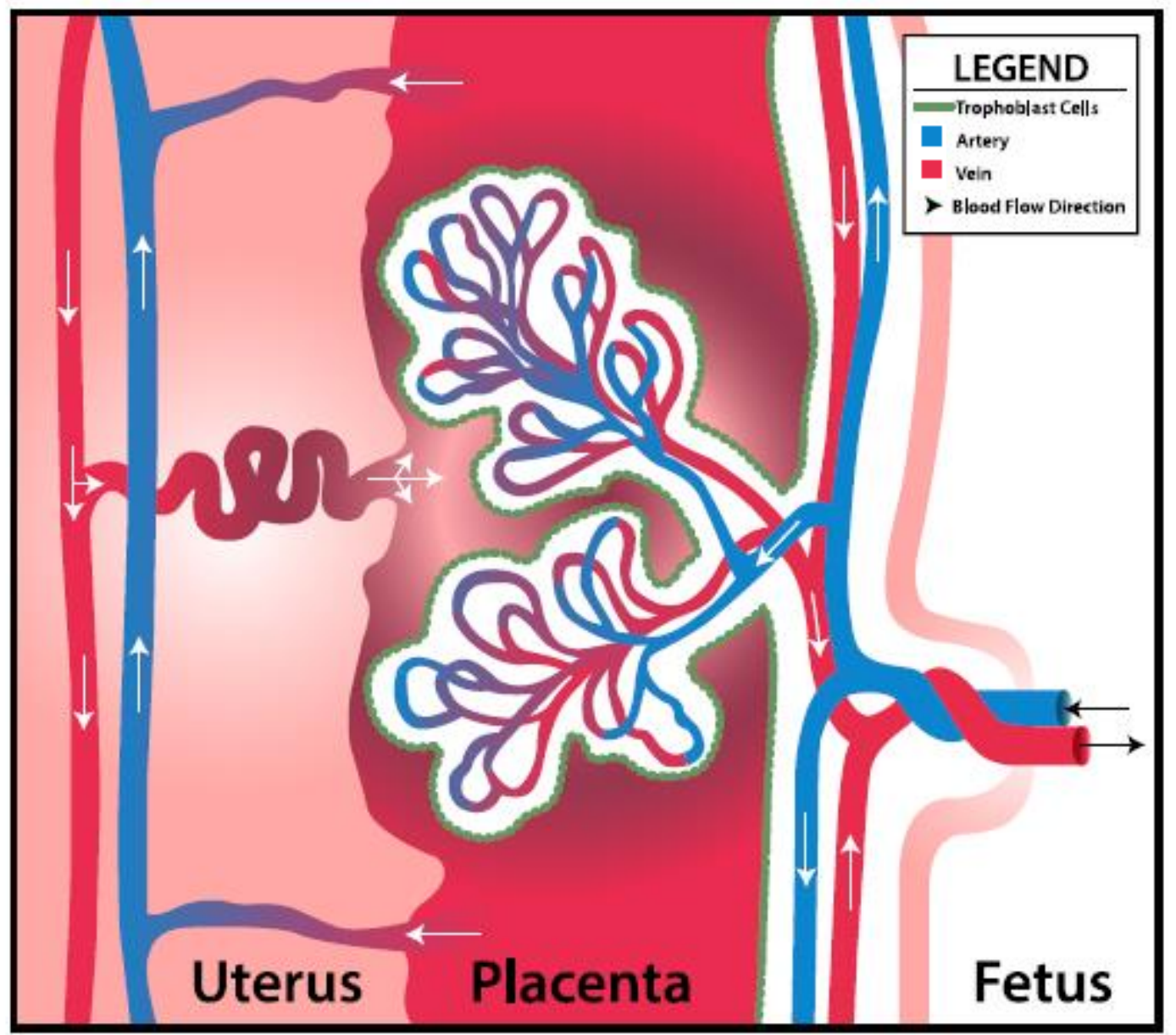




\section{Chapter 2}

Heterogeneous Vascular Bed Responses to Pulmonary Titanium Dioxide Nanoparticle Exposure

\section{Running Title: Vascular Gradient Response to Nanoparticles}

Citation: Abukabda, A. B., Stapleton, P. A., McBride, C. R., Yi, J., \& Nurkiewicz, T. R. (2017). Heterogeneous Vascular Bed Responses to Pulmonary Titanium Dioxide Nanoparticle Exposure. Frontiers in Cardiovascular Medicine, 4. doi:10.3389/fcvm.2017.00033

\section{Authors:}

Abukabda, A.B. ${ }^{1,2}$

Stapleton, P.A. ${ }^{1,2}$

McBride, C.R. ${ }^{1,2}$

Yi, $J^{1,2}$

Nurkiewicz, T.R. ${ }^{1,2}$

\section{Affiliations:}

${ }^{1}$ Department of Physiology and Pharmacology, West Virginia University School of Medicine, Morgantown, WV, USA.

${ }^{2}$ Department of Pharmacology and Toxicology, Rutgers University, Piscataway, NJ, USA. 


\section{Abstract:}

A growing body of research links engineered nanomaterial (ENM) exposure to adverse cardiovascular endpoints. The purpose of this study was to evaluate the impact of ENM exposure on vascular reactivity in discrete segments so that we may determine the most sensitive levels of the vasculature where these negative cardiovascular effects are manifest. We hypothesized that acute nano- $\mathrm{TiO}_{2}$ exposure differentially affects reactivity with a more robust impairment in the microcirculation. Sprague-Dawley rats (8-10 weeks) were exposed to nano- $\mathrm{TiO}_{2}$ via intratracheal instillation $(20 \mu \mathrm{g}, 100 \mu \mathrm{g}$, or $200 \mu \mathrm{g}$ suspended per $250 \mu \mathrm{l}$ of vehicle) 24 hours prior to vascular assessments. A serial assessment across distinct compartments of the vascular tree was then conducted. Wire myography was used to evaluate macrovascular active tension generation specifically in the thoracic aorta, the femoral artery and $3^{\text {rd }}$ order mesenteric arterioles. Pressure myography was used to determine vascular reactivity in $4^{\text {th }}$ and $5^{\text {th }}$ order mesenteric arterioles. Vessels were treated with phenylephrine (PE), acetylcholine (ACh), and sodium nitroprusside (SNP).

Nano- $\mathrm{TiO}_{2}$ exposure decreased endothelium-dependent relaxation in the thoracic aorta and femoral arteries assessed via ACh by $54 \pm 12 \%$, and $25 \pm 6 \%$, respectively. Relaxation of third order mesenteric arterioles was impaired by $100 \mu \mathrm{g}$ and $20 \mu \mathrm{g}$ nano-TiO2 exposures with mean reductions of $50 \pm 9 \%$ and $68 \pm 9 \%$. Cholinergic reactivity of $4^{\text {th }}$ and $5^{\text {th }}$ order mesenteric arterioles was negatively affected by nano- $\mathrm{TiO}_{2}$ with diminished dilations of $83 \pm 13 \%$ after exposure to 200 $\mu \mathrm{g}$ nano- $\mathrm{TiO}_{2}, 43 \pm 13 \%$ after $100 \mu \mathrm{g}$ nano-TiO2, and $49 \pm 13 \%$ after $20 \mu \mathrm{g}$ nano- $\mathrm{TiO}_{2}$.

Endothelium-independent relaxation was impaired in the thoracic aorta by $34 \pm 3 \%$ induced by exposure to $200 \mu \mathrm{g}$ nano- $\mathrm{TiO}_{2}$ and a reduction in response of $49 \pm 3 \%$ caused by $100 \mu \mathrm{g}$ nano- 
$\mathrm{TiO}_{2}$. Femoral artery response was reduced by $18 \pm 5 \%$, while $3^{\text {rd }}$ order mesenteric arterioles were negatively affected by $20 \mu \mathrm{g}$ nano- $\mathrm{TiO}_{2}$ with a mean decrease in response of $38 \pm 10 \%$. This is the first study to directly compare the differential effect of ENM exposure on discrete anatomical segments of the vascular tree. Pulmonary ENM exposure produced macro- and microvascular dysfunction resulting in impaired responses to endothelium-dependent, endothelium-independent, and adrenergic agonists with a more robust dysfunction at the microvascular level. These results provide additional evidence of an endothelium-dependent and -independent impairment in vascular reactivity.

Keywords: engineered nanomaterials, titanium dioxide, cardiovascular system, microcirculation, endothelium 


\section{Introduction:}

Engineered nanomaterials (ENM) are most commonly defined as a homogeneous mixture of anthropogenic materials possessing at least one dimension less than or equal to $100 \mathrm{~nm}$ [6] and have historically been used in inhalation toxicology research as surrogates for environmental air pollution studies to model ultrafine particulate matter exposures [23,30]. The continued development of novel ENM with diverse physicochemical properties represents a growing economic sector. Presently, the nanotechnology industry is one of the fastest growing markets with an estimated projected worth of $\$ 75.8$ billion by 2020 [20]. This increased prevalence of ENM not only continues concerns over their safety and the potential adverse health outcomes that may occur because of prolonged, unmitigated exposure but also warrants more thorough toxicological assessments of their potential deleterious systemic effects.

Titanium dioxide (nano- $\mathrm{TiO}_{2}$ ) is amongst the most widely used nanomaterials, its optical properties allow it to be used in consumer products such as topical sunscreens and cosmetics, as well as industrial components including paints [36]. It is used commonly as a photocatalyst, catalyst carrier, heat stabilizer and as a food additive [44]. Nano- $\mathrm{TiO}_{2}$ may also be used as a drug carrier in nanomedicine [25].

Pulmonary nano- $\mathrm{TiO}_{2}$ exposure has been associated with systemic inflammation in vivo $[5,11,43]$, with dose-dependent overt inflammation characterized by a marked increase in proinflammatory mediators and lung toxicity $[15,43]$. 
The circulatory system can be grossly divided into two major components based on structure and location: the macrocirculation and the microcirculation. The macrocirculation refers to the conduit arteries and veins which connect the heart with the systemic organs and function as pressure and blood reservoirs respectively. The microcirculation is a broad term encompassing all the vascular tissue within a specific organ, including arterioles, the capillary network, and venules [27]. Its fundamental function is the maintenance of an ideal internal environment for the controlled exchange of nutrients and metabolic waste with the adjacent tissues. For this study, conduit arteries and arterioles were chosen because they are major hemodynamic regulators and key players in the maintenance of blood pressure, flow distribution and tissue perfusion $[28,29]$.

The endothelium is a regulator of vascular homeostasis and is extremely sensitive to changes in blood composition and hemodynamics [18]. Consisting of a monolayer of cells that functions as a physical barrier for the exchange of materials between blood and tissues [4], it is also responsible for the secretion of a variety of molecules, including prostacyclins [7], clotting factors [31], and ectonucleotidases [26] that are important for the regulation of blood coagulation and platelet function. Endothelial cells also coordinate the recruitment of immune cells to sites of injury or infection via both the production and release of cytokines [21], and the expression of specific cell adhesion molecules on their apical surface that mediate leukocyte attachment and extravasation $[9,10,34,37]$. While common to all vascular segments, endothelial cells show significant morphological and functional heterogeneity throughout the vasculature, between different organs, and between neighboring endothelial cells of the same organ and blood vessel type [2]. These phenotypic differences may account for the differential and vascular-bed specific responses of endothelial cells to specific toxicants. 
We have previously established that nano- $\mathrm{TiO}_{2}$ exposure is associated with systemic vascular dysfunction $[12,19,38]$. However, the relative sensitivity to ENM of each vascular segment is not known. Therefore, the aim of this study is to assess the effects of three occupationally relevant nano- $\mathrm{TiO}_{2}$ concentrations on both the macrocirculation and microcirculation from a functional perspective via wire and pressure myography. $100 \mu \mathrm{g}$ of nano- $\mathrm{TiO}_{2}$ was selected based on previous work conducted by our group [23]. Additionally, $200 \mu \mathrm{g}$ and $20 \mu \mathrm{g}$ were chosen for this study to identify the maximum and minimum observed dose responses. The most sensitive vascular level to nano- $\mathrm{TiO}_{2}$ exposure was subsequently determined. Based on our previous results we hypothesize that nano- $\mathrm{TiO}_{2}$ exposure impairs endothelium-dependent responsiveness and that this effect is more pronounced in the microcirculation. 


\section{Methods:}

\section{Nanomaterial Characterization}

Nano- $\mathrm{TiO}_{2}$ P25 powder, obtained from Evonik (Aeroxide $\mathrm{TiO}_{2}$, Parsippany, $\mathrm{NJ}$ ), has previously been shown to be a mixture composed primarily of anatase $(80 \%)$ and rutile $(20 \%) \mathrm{TiO}_{2}$, with a primary particle size of $21 \mathrm{~nm}$ and a surface area of $48.08 \mathrm{~m}^{2} / \mathrm{g}[23,32,33]$.

\section{Experimental Animals and Exposure}

Male (8-10 weeks) Sprague-Dawley rats were purchased from Hilltop Laboratories (Scottdale, PA) and housed at WVU with 12:12 h light-dark cycle and regulated temperature. Rats were allowed ad libitum access to food and water. All procedures were approved by the Institutional Animal Care and Use Committee of West Virginia University and abide by the standards set forth in the "Guide for Care and Use of Laboratory Animals" of the National Research Council of the National Academies.

Bolus doses of nano- $\mathrm{TiO}_{2}(20 \mu \mathrm{g}, 100 \mu \mathrm{g}$ and $200 \mu \mathrm{g})$ were suspended in $250 \mu \mathrm{L}$ of vehicle (Normosol and 5\% fetal bovine serum) for intratracheal instillation (IT) 24 hours prior to experimentation. Nano- $\mathrm{TiO}_{2}$ suspensions were sonicated over ice for 1 minute to ensure increased dispersion. Sprague-Dawley rats were anesthetized using $5 \%$ isoflurane, placed on a mounting stand and IT with $200 \mu \mathrm{L}$ of vehicle (negative control) or the nano- $\mathrm{TiO}_{2}$ suspensions. 


\section{Mean Arterial Pressure (Map) Acquisition}

Rats were anesthetized with isoflurane gas (5\% induction, 3-3.5\% maintenance) and placed on a heating pad to maintain a $37^{\circ} \mathrm{C}$ rectal temperature. The trachea was intubated to ensure an open airway and the right carotid artery was cannulated to acquire MAP. PowerLab830 (AD Instruments) was used to record MAP.

\section{Wire Myography Arterial Ring Preparation}

The thoracic aorta, common femoral artery, $3^{\text {rd }}$ order mesenteric arterioles were dissected and immediately placed in ice cold Ringer`s solution. 2-3 mm segments were cut and mounted in each wire myograph chamber (AD Instruments - DMT 620M) containing $5 \mathrm{~mL}$ of physiological salt solution (PSS; $119 \mathrm{mM} \mathrm{NaCl}, 4.7 \mathrm{mM} \mathrm{KCl}, 1.18 \mathrm{mM} \mathrm{KH}_{2} \mathrm{PO}_{4}, 1.17 \mathrm{mM} \mathrm{MgSO}_{4}, 2.5 \mathrm{mM}, \mathrm{CaCl}_{2}$, $25 \mathrm{mM} \mathrm{NaHCO}_{3}, 0.027 \mathrm{mM}$ EDTA, and $5.5 \mathrm{mM}$ glucose) at $\mathrm{pH} 7.4$ and bubbled with $95 \% \mathrm{O}_{2}$ / $5 \% \mathrm{CO}_{2}$ at $37^{\circ} \mathrm{C}$. After 30 minutes of equilibration, maximum contractile response was determined by using high-potassium PSS (K+PSS; $123.7 \mathrm{mM} \mathrm{KCl,} 1.18 \mathrm{mM} \mathrm{KH}_{2} \mathrm{PO}_{4}, 1.17 \mathrm{mM} \mathrm{MgSO}_{4}, 2.5$ $\mathrm{mM}, \mathrm{CaCl}_{2}, 25 \mathrm{mM} \mathrm{NaHCO}_{3}, 0.027 \mathrm{mM}$ EDTA, and $5.5 \mathrm{mM}$ glucose). Vessels were then washed twice with PSS and allowed to relax until initial tension was reached. Contractile responses were determined via cumulative additions of $50 \mu \mathrm{L}$ of phenylephrine (PE- $\left.1 \times 10^{-9}-1 \times 10^{-4} \mathrm{M}\right)$. Relaxation responses were evaluated via cumulative addition of $50 \mu \mathrm{L}$ acetylcholine (ACh) or sodium nitroprusside (SNP) $\left(1 \times 10^{-9}-1 \times 10^{-4} \mathrm{M}\right)$.

\section{Statistics}

Point-to-point differences in the dose-response curves were evaluated using two-way repeated measures analysis of variance (ANOVA) with a Tukey`s post-hoc analysis when significance was 
found. The slopes of the dose response curves were determined through nonlinear regression. The animal characteristics, vessel characteristics and dose response curve slopes were analyzed using a one-way ANOVA with a Tukey post-hoc analysis when significance was found. All statistical analysis was completed with GraphPad Prism 5 (San Diego, CA) and SigmaPlot 11.0 (San Jose, CA). Significance was set at $p<0.05, n$ is the number of arterioles, and $\mathrm{N}$ is the number of animals.

\section{Wire Myography Calculations}

Maximum tension was defined as the tension developed by the vessels with $\mathrm{K}+\mathrm{PSS}$. Tension with agonists (ACh, SNP or PE) was recorded and the percentage of maximum tension generation was calculated using the following formula:

$$
\text { Percentage Maximum Tension (\%) }=\left(\frac{\text { Tension with agonists }(\mathrm{mN})}{\text { Maximum tension }(\mathrm{mN})}\right) \times 100 \%
$$

The response of the different arterial branching orders was quantitatively assessed and compared by using the slope of the individual dose-response curves as previously reported [45].

\section{Pressure Myogaphy Arteriolar Preparation}

The mesentery was excised and placed in a dissecting dish with PSS maintained at $4{ }^{\circ} \mathrm{C}$. $4^{\text {th }}$ and $5^{\text {th }}$ order mesenteric arterioles were isolated, transferred to a vessel chamber, cannulated between two glass pipettes (outer diameter $60 \mu \mathrm{m}$ ), and secured by means of silk sutures in the vessel chamber (Living Systems Instrumentation, Burlington, VT). The chamber was superfused with fresh oxygenated $\left(5 \% \mathrm{CO}_{2} / 21 \% \mathrm{O}_{2}\right) \mathrm{PSS}$ and warmed to $37^{\circ} \mathrm{C}$. Arterioles were pressurized 
to $60 \mathrm{~mm} \mathrm{Hg}$ using a servo control system and extended to their in situ length. Internal and external arteriolar diameters were measured using video calipers (Colorado Video, Boulder, CO).

\section{Arteriolar Reactivity}

Arterioles were equilibrated for 1 hour prior to experimentation. Arterioles with $<20 \%$ spontaneous tone were not analyzed. Contractile responses were determined via cumulative additions of $50 \mu \mathrm{L}$ of phenylephrine (PE) $\left(1 \times 10^{-9}-1 \times 10^{-4}\right) \mathrm{M}$. Relaxation responses were evaluated via cumulative addition of $50 \mu \mathrm{L}$ acetylcholine (ACh) or sodium nitroprusside (SNP) $\left(1 \times 10^{-9}-1 \times 10^{-}\right.$ $\left.{ }^{4} \mathrm{M}\right)$. The steady state diameter of the vessel was recorded for at least 2 min after each dose. After each dose curve was completed, the vessel chamber was washed to remove excess chemicals by carefully removing the superfusate and replacing it with fresh warmed oxygenated PSS. After all experimental treatments were complete, the PSS was replaced with $\mathrm{Ca}^{2}+$-free PSS until maximum passive diameter was established.

\section{Pressure Myography Calculations}

All measurements were conducted during steady state conditions, with steady state being defined as the interval during which blood vessels maintained a stable tone for 1 minute or more. Vessels were washed twice with PSS between individual dose - response treatments and allowed approximately 10 minutes for equilibration or until original tone was developed. Data are expressed as means \pm standard error. Spontaneous tone was calculated by the following equation:

$$
\text { Spontaneous tone }(\%)=\left\{\frac{(D m-D i)}{D i}\right\} \times 100
$$


, where $\mathrm{Dm}$ is the maximal diameter and $\mathrm{Di}$ is the initial steady state diameter recorded prior to the experiment. Active responses to pressure were normalized to the maximal diameter using the following formula:

$$
\text { Normalized diameter }=D s s / D m
$$

, where Dss is the steady state diameter recorded during each pressure change. The experimental responses to $\mathrm{ACh}$, and SNP are expressed using the following equation:

$$
\text { Diameter }(\text { percent maximal diameter })=\left\{\frac{(\text { Dss }- \text { Dcon })}{(D m-D c o n)}\right\} \times 100
$$

, where DCon is the control diameter recorded prior to the dose curve, DSS is the steady state diameter at each dose of the curve. The experimental response to PE is expressed using the following equation:

$$
\text { Diameter (percent maximal diameter })=\left\{\frac{(\text { Dcon }- \text { Dss })}{(\text { Dcon })}\right\} \times 100
$$

Wall thickness (WT) was calculated from the measurement of both inner (ID) and outer (OD) steady state arteriolar diameters at the end of the $\mathrm{Ca} 2+$ free wash using the following equation:

$$
W T=(O D-I D) / 2
$$

Wall-to-lumen ratio (WLR) was calculated using the following equation:

$$
W L R=W T / I D
$$




\section{Results:}

\section{Animal and Vessel Characteristics}

No significant changes were observed in age, MAP, heart rate and body weight between control and exposure groups (Table 1). Active inner diameter of $4^{\text {th }}$ and $5^{\text {th }}$ order mesenteric arterioles exposed to $200 \mu \mathrm{g}$ of nano- $\mathrm{TiO}_{2}(134 \pm 11 \mu \mathrm{m})$ and $100 \mu \mathrm{g}$ of nano- $\mathrm{TiO}_{2}(128 \pm 19 \mu \mathrm{m})$ were significantly different from control values $(115 \pm 10 \mu \mathrm{m})$, while passive diameter remained unchanged. This increase in diameter was associated with a decreased initial vascular tone (Table 2).

\section{Endothelium-Dependent Reactivity}

$200 \mu \mathrm{g}$ of nano- $\mathrm{TiO}_{2}$ significantly impaired endothelium-dependent relaxation of thoracic aorta (Figure 1 A) with a mean decrease in response to ACh of $54 \pm 12 \%$. No significant effects were seen at $100 \mu \mathrm{g}$ and $20 \mu \mathrm{g}$ of nano- $\mathrm{TiO}_{2}$ (Figure $\left.1 \mathbf{A}\right)$.

Femoral arteries from exposed animals showed a reduced cholinergic response solely at the lowest exposure concentration $\left(20 \mu \mathrm{g}\right.$ nano- $\mathrm{TiO}_{2} / 250 \mu \mathrm{L}$ of vehicle) of $25 \pm 6 \%$ (Figure $1 \mathrm{~B}$ ). Further, $100 \mu \mathrm{g}$ and $20 \mu \mathrm{g}$ nano- $\mathrm{TiO}_{2}$ exposure resulted in a mean reduction in relaxation of third order mesenteric arterioles of $50 \pm 9 \%$ and $68 \pm 9 \%$ respectively (Figure $1 \mathrm{C}$ ). Lastly, cholinergic reactivity of $4^{\text {th }}$ and $5^{\text {th }}$ order mesenteric arterioles was negatively affected by nano- $\mathrm{TiO}_{2}$ with diminished dilations of $83 \pm 13 \%$ after exposure to $200 \mu$ g nano- $\mathrm{TiO}_{2}, 43 \pm 13 \%$ after $100 \mu \mathrm{g}$ nano$\mathrm{TiO}_{2}$, and $49 \pm 13 \%$ after $20 \mu \mathrm{g}$ nano- $\mathrm{TiO}_{2}$ (Figure $\left.1 \mathrm{D}\right)$.

In order to consider the fullest scope of the biological response across the discrete entities of the vasculature, the slope of the individual dose-response curves was utilized as a means of 
numerically quantifying vascular reactivity (Figure 2) as previously shown $[25,45]$. Exposure to $200 \mu \mathrm{g}$ of nano- $\mathrm{TiO}_{2}$ decreased sensitivity to $\mathrm{ACh}$ from $16 \pm 1 \mathrm{M}^{-1}$ to $4 \pm 1 \mathrm{M}^{-1}$. Similarly, vascular reactivity of femoral arteries was diminished from the control value of $12 \pm 2 \mathrm{M}^{-1}$ to $8 \pm 4 \mathrm{M}^{-1}$ and $7 \pm 3 \mathrm{M}^{-1}$ with $200 \mu \mathrm{g}$ and $100 \mu \mathrm{g}$ nano- $\mathrm{TiO}_{2}$ respectively. Third order mesenteric arterioles showed a dose dependent reduction in ACh sensitivity from control levels $\left(17 \pm 3 \mathrm{M}^{-1}\right)$ with 100 $\mu \mathrm{g}$ nano- $\mathrm{TiO}_{2}$ having the greatest deleterious effect $\left(3 \pm 1 \mathrm{M}^{-1}\right)$, while $200 \mu \mathrm{g}$ nano- $\mathrm{TiO}_{2}$ and 20 $\mu \mathrm{g}$ nano- $\mathrm{TiO}_{2}$ reduced responsiveness to $7 \pm 3 \mathrm{M}^{-1}$ and $3 \pm 1 \mathrm{M}^{-1} \cdot 4^{\text {th }}$ and $5^{\text {th }}$ mesenteric arterioles showed the greatest decrease in vascular reactivity from controls $\left(15 \pm 2 \mathrm{M}^{-1}\right)$ particularly with $200 \mu \mathrm{g}$ nano- $\mathrm{TiO}_{2}\left(-12 \pm 5 \mathrm{M}^{-1}\right)$ and $20 \mu \mathrm{g}$ nano- $\mathrm{TiO}_{2}\left(-2 \pm 1 \mathrm{M}^{-1}\right)$, while vessels exposed to 100 $\mu \mathrm{g}$ nano- $\mathrm{TiO}_{2}$ showed slight $\mathrm{ACh}$ sensitivity $\left(1 \pm 0.4 \mathrm{M}^{-1}\right)$. These results provide additional evidence of an endothelium-dependent effect associated with nanomaterial exposure. The heterogeneity in impairment across the vasculature indicates the microcirculation as the prime site where these effects are most markedly manifest.

\section{a-adrenergic Sensitivity}

In contrast to endothelium-dependent relaxation, no point to point differences were seen in $\alpha$ adrenergic responses across all levels of the vasculature between nano- $\mathrm{TiO}_{2}$ exposed and control groups (Figure 3 A-D). However, slope analysis, wherein the entire continuum of the vascular PE responses is assessed, revealed significant differences in vascular sensitivity induced by nano- $\mathrm{TiO}_{2}$ (Figure 4). Aortic PE sensitivity was increased by exposure to $100 \mu \mathrm{g}$ nano- $\mathrm{TiO}_{2}(11$ $\left.\pm 1 \mathrm{M}^{-1}\right)$ with respect to control values $\left(14 \pm 0.6 \mathrm{M}^{-1}\right)$. Significantly augmented adrenergic responses were seen in the femoral artery with $100\left(9 \pm 3 \mathrm{M}^{-1}\right)$ and $20 \mu \mathrm{g}$ nano- $\mathrm{TiO}_{2}\left(9 \pm 2 \mathrm{M}^{-1}\right)$. Also, $200 \mu \mathrm{g}$ nano- $\mathrm{TiO}_{2}\left(6 \pm 2 \mathrm{M}^{-1}\right)$ and $20 \mu \mathrm{g}$ nano- $\mathrm{TiO}_{2}\left(6 \pm 1 \mathrm{M}^{-1}\right)$ caused increased vascular reactivity of $3^{\text {rd }}$ order mesenteric arterioles relative to controls $\left(2 \pm 3 \mathrm{M}^{-1}\right)$. Lastly, $4^{\text {th }}$ and $5^{\text {th }}$ mesenteric arterioles from exposed animals showed a clear pro-constrictive effect particularly at 
$200 \mu \mathrm{g}$ nano- $\mathrm{TiO}_{2}\left(20 \pm 2 \mathrm{M}^{-1}\right)$ and $20 \mu \mathrm{g}\left(24 \pm 2 \mathrm{M}^{-1}\right)$, with $100 \mu \mathrm{g}\left(20 \pm 2 \mathrm{M}^{-1}\right)$ leading to responses similar to controls $\left(16 \pm 1 \mathrm{M}^{-1}\right)$ (Figure 4). This effect is consistent with previous work [12,42] and confirms a potential effect on sympathetic tone, associated with ENM exposure.

\section{Endothelium-Independent Reactivity}

Endothelium-independent relaxation assessed via the spontaneous NO donor SNP was impaired in the thoracic aorta with a mean decrease in response of $34 \pm 3 \%$ induced by exposure to 200 $\mu \mathrm{g}$ nano- $-\mathrm{TiO}_{2}$ and a reduction in response of $49 \pm 3 \%$ caused by $100 \mu \mathrm{g}$ nano-TiO 2 (Figure $5 \mathrm{~A}$ ). Similarly, the response of the femoral artery to SNP was reduced by $18 \pm 5 \%$ (Figure 5 B). $3^{\text {rd }}$ order mesenteric arterioles were negatively affected by $20 \mu \mathrm{g}$ nano- $\mathrm{TiO}_{2}$ with a mean decrease in response of $38 \pm 10 \%$ (Figure $\mathbf{5} \mathbf{C}$ ). It is interesting to note that slope analysis demonstrated a decrease in SNP sensitivity only in thoracic aorta at $200 \mu \mathrm{g}\left(21 \pm 2 \mathrm{M}^{-1}\right)$ and $100 \mu \mathrm{g}\left(19 \pm 2 \mathrm{M}^{-}\right.$ 1) nano- $-\mathrm{TiO}_{2}$ compared to control $\left(26 \pm 3 \mathrm{M}^{-1}\right)$ (Figure 6). Similarly, $4^{\text {th }}$ and $5^{\text {th }}$ mesenteric arteriolar sensitivity to SNP was significantly reduced from $\left(18 \pm 2 \mathrm{M}^{-1}\right)$ to $\left(16 \pm 2 \mathrm{M}^{-1}\right)$ by exposure to $20 \mu \mathrm{g}$ nano- $\mathrm{TiO}_{2}$. This is consistent with previous findings where impairments in endotheliumindependent dilation in the microcirculation is not noted $[13,22,39]$. 


\section{Discussion:}

This is the first study to directly compare the differential effect of ENM exposure on discrete anatomical segments of the vascular tree. Pulmonary ENM exposure produced macro- and microvascular dysfunction resulting in impaired responses to endothelium-dependent, endothelium-independent and adrenergic agonists. The major finding of this paper is that acute nano- $\mathrm{TiO}_{2}$ pulmonary exposure results in a more robust dysfunction at the microvascular level, and this is not simply because arterioles possess greater reactivity than arteries.

The microcirculation is critical in the regulation of the vascular internal environment for the controlled exchange of nutrients and metabolic waste with the adjacent tissues. Specifically, arterioles are major hemodynamic regulators and key players in the maintenance of blood pressure and flow distribution [28,29]. Disruptions in microvascular function are commonly associated with health consequences such as hypertension and diabetes, and this is largely due to endothelial dysfunction [35].

The pronounced effect of ENM exposure on the microcirculation may be due to the propensity of nanoparticles to accumulate preferentially in this vascular compartment. Considerable evidence exists suggesting that after the initial exposure, nanomaterials tend to translocate and accumulate systemically $[16,17]$. Due to the existing hydrodynamic influences present within the microcirculation, it has been hypothesized that ENM deposition would likely be highest in the arterioles [41]. Within arterioles, ENM may directly impair vascular function via generation of free radicals, decreasing NO bioavailability, NO synthase uncoupling, or by altering sympathetic tone. 
Cholinergic activity was assessed using ACh. In normal conditions, ACh activates endothelial NO synthase (eNOS) by stimulating the release of intracellular calcium. L-Arginine is converted to L-citrulline by eNOS with the production of NO. eNOS requires a number of cofactors, including $\mathrm{NADPH}, \mathrm{FAD}$, calcium, calmodulin, and $\mathrm{BH}_{4}$. $\mathrm{NO}$ subsequently diffuses into vascular smooth muscle cells and stimulates guanylate cyclase, ultimately resulting in cGMP production and vasodilation. In this study, reduced reactivity was seen in the thoracic aorta, femoral artery, $3^{\text {rd }}$ order mesenteric arterioles and $4^{\text {th }} / 5^{\text {th }}$ mesenteric arterioles. These effects are consistent with previous results obtained by our group and others $[24,40,42]$. It is interesting to note, however, that exposure to $200 \mu \mathrm{g}$ nano- $\mathrm{TiO}_{2}$ resulted in a complete reversal of the vasodilatory response by $4^{\text {th }} / 5^{\text {th }}$ mesenteric arterioles, potentially indicating severe irreparable damage to the endothelial layer.

The endothelium is a major regulatory component common to all levels of the vasculature and plays a critical role in the control of vascular tone, the inflammatory response, maintenance and regulation of blood fluidity, permeability, and angiogenesis [3]. Due to the significant role played in vascular homeostasis, it is becoming increasingly recognized that the endothelium is involved in most disease conditions, either as a primary determinant of pathophysiology or as a result of collateral damage [1].

No point-to-point impairments were seen in the response to the $\alpha$-adrenergic agonist PE. However, when considering the whole continuum of the vascular responses to this agonist, a prevalent increase in sensitivity, particularly at the microvascular level was observed (Figure 4), consistent with previous work [12,42]. 
Lastly, endothelium-independent dysfunction was observed primarily in the macrocirculation, including the thoracic aorta, femoral artery, and $3^{\text {rd }}$ order mesenteric arterioles (Figures 5 and 6). This differential effect on vascular smooth muscle response in the macrocirculation may be caused by the turbulent flow patterns characteristic of this segment which contribute to an increased likelihood of deposition and impaction of ENM agglomerates particularly at large artery bifurcations [41].

Dysfunction in vascular smooth muscle following particulate matter exposure was first reported in pulmonary arteries by Courtois et al. [8]. Previous work attributed this effect to a proinflammatory response triggered by particulate matter exposure, while other studies linked vascular dysfunction to an increase in oxidative stress [14].

Historically, the adverse systemic effects associated with pulmonary ENM exposure have been ascribed to a host inflammatory response, direct particle-tissue interactions and/or autonomic dysregulation [30]. The observed macro- and microvascular dysfunction could be associated with either impaired NO production, a decrease in NO bioavailability caused by the generation of reactive oxygen species within the vascular wall, a shift in arachidonic acid metabolites or vascular smooth muscle impairment or a combination of these mechanisms. It is outside the scope of this study to determine the mechanisms underlying the vascular endpoints herein reported, and future experiments are necessary to elucidate these mechanisms.

While the results of this study provide further indications of the microvascular effects associated with ENM, it is admittedly not without its limitations. Wire myography assesses the tension generated by vascular rings under isometric tension and therefore caution should be taken when 
interpreting the results obtained. This is particularly so for conduit arteries, in which changes in wall tension do not explicitly translate into vasomotion. Further, the use of in vitro techniques to evaluate vascular reactivity eliminates neurogenic and hormonal influences which also play an important role in the mechanism of ENM cardiovascular toxicity. Therefore, future experiments utilizing ex vivo preparations (such as intravital microscopy) may be more appropriate to obtain a more thorough assessment. 
Figure Legends:

Figure 1. Endothelium - dependent dilation is impaired by intratracheal instillation of nano$\mathrm{TiO}_{2}$. ACh-induced vascular reactivity in A) Aorta, B) Femoral artery, C) $3^{\text {rd }}$ order mesenteric arterioles, and D) $4^{\text {th }}$ and $5^{\text {th }}$ mesenteric arterioles $(n=10-11)$. Statistics were analyzed with twoway ANOVA (P $\leq 0.05)$. * Sham control group vs. nano- $\mathrm{TiO}_{2}$ exposed groups.

Figure 2. ACh sensitivity and vascular reactivity are decreased by nano- $\mathrm{TiO}_{2}$ exposure. Bar graphs showing the individual slope values for sham control and exposed vessels $(n=9-$ 11). Statistics were analyzed with two-way ANOVA ( $P \leq 0.05)$. * Sham control group vs. nano$\mathrm{TiO}_{2}$ exposed groups.

Figure 3. $\alpha$-adrenergic response to $\mathrm{PE}$ is not affected by nano- $\mathrm{TiO}_{2}$. PE response in A) Aorta, B) Femoral artery, C) $3^{\text {rd }}$ order mesenteric arterioles, and D) $4^{\text {th }}$ and $5^{\text {th }}$ mesenteric arterioles $(\mathrm{n}=$ 10-11). Statistics were analyzed with two-way ANOVA (P $\leq 0.05)$. * Sham control group vs. nano$\mathrm{TiO}_{2}$ exposed groups.

Figure 4. PE sensitivity are augmented by nano- $\mathrm{TiO}_{2}$ exposure. Bar graphs showing the individual slope values for sham-control and exposed vessels $(n=10-11)$. Statistics were analyzed with two-way ANOVA ( $\mathrm{P} \leq 0.05)$. * Sham control group vs. nano- $\mathrm{TiO}_{2}$ exposed groups.

Figure 5. Endothelium-Independent dilation and vascular smooth muscle function is not impaired by nano- $\mathrm{TiO}_{2}$ exposure. SNP response in A) Aorta, B) Femoral artery, C) $3^{\text {rd }}$ order mesenteric arterioles, and D) $4^{\text {th }}$ and $5^{\text {th }}$ mesenteric arterioles $(n=10-11)$. Statistics were analyzed with two-way ANOVA $(\mathrm{P} \leq 0.05)$. * Sham control group vs. nano- $\mathrm{TiO}_{2}$ exposed groups. 
Figure 6: SNP-induced vascular reactivity is unaffected by nano- $\mathrm{TiO}_{2}$ exposure. Bar graphs showing the individual slope values for sham-control and exposed vessels $(n=10-11)$. Statistics were analyzed with two-way ANOVA $(\mathrm{P} \leq 0.05)$. ${ }^{*}$ Sham control group vs. nano- $\mathrm{TiO}_{2}$ exposed groups. 


\section{Reference List:}

[1] Aird WC. Endothelial cell heterogeneity. Crit Care Med 2003; 31 S221-S230

[2] Aird WC. Mechanisms of endothelial cell heterogeneity in health and disease. Circ Res 2006; 98 159-62

[3] Aird WC. Endothelial cell heterogeneity. Cold Spring Harb Perspect Med 2012; 2 a006429

[4] Baldwin AL, Thurston G. Mechanics of endothelial cell architecture and vascular permeability. Crit Rev Biomed Eng 2001; 29 247-78

[5] Bonner JC, Silva RM, Taylor AJ, et al. Interlaboratory evaluation of rodent pulmonary responses to engineered nanomaterials: the NIEHS Nano GO Consortium. Environ Health Perspect 2013; $121676-82$

[6] Borm P, Klaessig FC, Landry TD, et al. Research strategies for safety evaluation of nanomaterials, part V: role of dissolution in biological fate and effects of nanoscale particles. Toxicol Sci 2006; 90 23-32

[7] Cines DB. Glycoprotein Ilb/llla antagonists: potential induction and detection of drugdependent antiplatelet antibodies. Am Heart J 1998; 135 S152-S159

[8] Courtois A, Andujar P, Ladeiro Y, et al. Impairment of NO-dependent relaxation in intralobar pulmonary arteries: comparison of urban particulate matter and manufactured nanoparticles. Environ Health Perspect 2008; 116 1294-9

[9] Ellies LG, Sperandio M, Underhill GH, et al. Sialyltransferase specificity in selectin ligand formation. Blood 2002; 100 3618-25 
[10] Forlow SB, Foley PL, Ley K. Severely reduced neutrophil adhesion and impaired host defense against fecal and commensal bacteria in CD18-/-P-selectin-/- double null mice. FASEB J 2002; 16 1488-96

[11] Hougaard KS, Jackson P, Jensen KA, et al. Effects of prenatal exposure to surface-coated nanosized titanium dioxide (UV-Titan). A study in mice. Part Fibre Toxicol 2010; 716

[12] Knuckles TL, Yi J, Frazer DG, et al. Nanoparticle inhalation alters systemic arteriolar vasoreactivity through sympathetic and cyclooxygenase-mediated pathways. Nanotoxicology 2012; 6 724-35

[13] LeBlanc AJ, Cumpston JL, Chen BT, et al. Nanoparticle inhalation impairs endotheliumdependent vasodilation in subepicardial arterioles. J Toxicol Environ Health A 2009; 72 1576-84

[14] LeBlanc AJ, Moseley AM, Chen BT, et al. Nanoparticle inhalation impairs coronary microvascular reactivity via a local reactive oxygen species-dependent mechanism. Cardiovasc Toxicol 2010; 10 27-36

[15] Ma-Hock L, Burkhardt S, Strauss V, et al. Development of a short-term inhalation test in the rat using nano-titanium dioxide as a model substance. Inhal Toxicol 2009; 21 102-18

[16] Mercer RR, Scabilloni JF, Hubbs AF, et al. Distribution and fibrotic response following inhalation exposure to multi-walled carbon nanotubes. Part Fibre Toxicol 2013; 1033

[17] Mercer RR, Scabilloni JF, Hubbs AF, et al. Extrapulmonary transport of MWCNT following inhalation exposure. Part Fibre Toxicol 2013; 1038

[18] Michiels C. Endothelial cell functions. J Cell Physiol 2003; 196 430-43 
[19] Minarchick VC, Stapleton PA, Porter DW, et al. Pulmonary cerium dioxide nanoparticle exposure differentially impairs coronary and mesenteric arteriolar reactivity. Cardiovasc Toxicol 2013; 13 323-37

[20] Mody VV, Siwale R, Singh A, Mody HR. Introduction to metallic nanoparticles. J Pharm Bioallied Sci 2010; 2 282-9

[21] Muller WA. Leukocyte-endothelial cell interactions in the inflammatory response. Lab Invest 2002; 82 521-33

[22] Nurkiewicz TR, Porter DW, Barger M, et al. Particulate matter exposure impairs systemic microvascular endothelium-dependent dilation. Environ Health Perspect 2004; 112 1299306

[23] Nurkiewicz TR, Porter DW, Hubbs AF, et al. Nanoparticle inhalation augments particledependent systemic microvascular dysfunction. Part Fibre Toxicol 2008; 51

[24] Nurkiewicz TR, Porter DW, Hubbs AF, et al. Pulmonary nanoparticle exposure disrupts systemic microvascular nitric oxide signaling. Toxicol Sci 2009; 110 191-203

[25] Palombo M, Deshmukh M, Myers D, et al. Pharmaceutical and toxicological properties of engineered nanomaterials for drug delivery. Annu Rev Pharmacol Toxicol 2014; 54 581-98

[26] Pearson JD, Carleton JS, Gordon JL. Metabolism of adenine nucleotides by ectoenzymes of vascular endothelial and smooth-muscle cells in culture. Biochem J 1980; 190 421-9

[27] Popel AS, Johnson PC. Microcirculation and Hemorheology. Annu Rev Fluid Mech 2005; $3743-69$ 
[28] Pries AR, Secomb TW. Rheology of the microcirculation. Clin Hemorheol Microcirc 2003; $29143-8$

[29] Pries AR, Secomb TW, Gaehtgens P. Biophysical aspects of blood flow in the microvasculature. Cardiovasc Res 1996; 32 654-67

[30] Rajagopalan S, Brook RD. The Indoor-Outdoor Air-Pollution Continuum and the Burden of Cardiovascular Disease: An Opportunity for Improving Global Health. Glob Heart 2012; 7 207-13

[31] Sadler JE. Thrombomodulin structure and function. Thromb Haemost 1997; 78 392-5

[32] Sager TM, Castranova V. Surface area of particle administered versus mass in determining the pulmonary toxicity of ultrafine and fine carbon black: comparison to ultrafine titanium dioxide. Part Fibre Toxicol 2009; 615

[33] Sager TM, Kommineni C, Castranova V. Pulmonary response to intratracheal instillation of ultrafine versus fine titanium dioxide: role of particle surface area. Part Fibre Toxicol 2008; 517

[34] Schober A, Manka D, von HP, et al. Deposition of platelet RANTES triggering monocyte recruitment requires P-selectin and is involved in neointima formation after arterial injury. Circulation 2002; 106 1523-9

[35] Schwartzkopff B, Mundhenke M, Strauer BE. Alterations of the architecture of subendocardial arterioles in patients with hypertrophic cardiomyopathy and impaired coronary vasodilator reserve: a possible cause for myocardial ischemia. J Am Coll Cardiol 1998; 31 1089-96 
[36] Silva RM, Teesy C, Franzi L, et al. Biological response to nano-scale titanium dioxide (TiO2): role of particle dose, shape, and retention. J Toxicol Environ Health A 2013; 76 953-72

[37] Smith ML, Smith MJ, Lawrence MB, Ley K. Viscosity-independent velocity of neutrophils rolling on p-selectin in vitro or in vivo. Microcirculation 2002; 9 523-36

[38] Stapleton PA, McBride CR, Yi J, Nurkiewicz TR. Uterine microvascular sensitivity to nanomaterial inhalation: An in vivo assessment. Toxicol Appl Pharmacol 2015; 288 420-8

[39] Stapleton PA, Minarchick VC, Cumpston AM, et al. Impairment of coronary arteriolar endothelium-dependent dilation after multi-walled carbon nanotube inhalation: a timecourse study. Int J Mol Sci 2012; 13 13781-803

[40] Stapleton PA, Nichols CE, Yi J, et al. Microvascular and mitochondrial dysfunction in the female F1 generation after gestational TiO2 nanoparticle exposure. Nanotoxicology 2015; $9941-51$

[41] Stapleton PA, Nurkiewicz TR. Vascular distribution of nanomaterials. Wiley Interdiscip Rev Nanomed Nanobiotechnol 2014; 6 338-48

[42] Vidanapathirana AK, Thompson LC, Mann EE, et al. PVP formulated fullerene (C60) increases Rho-kinase dependent vascular tissue contractility in pregnant Sprague Dawley rats. Reprod Toxicol 2014; 49 86-100

[43] Warheit DB, Webb TR, Reed KL, et al. Pulmonary toxicity study in rats with three forms of ultrafine-TiO2 particles: differential responses related to surface properties. Toxicology $2007 ; 23090-104$

[44] Weir A, Westerhoff $P$, Fabricius $L$, et al. Titanium dioxide nanoparticles in food and personal care products. Environ Sci Technol 2012; 46 2242-50 
[45] Wright CE, Angus JA. Effects of hypertension and hypercholesterolemia on vasodilatation in the rabbit. Hypertension 1986; 8 361-71 


\section{Table 1: Animal Characteristics}

\begin{tabular}{|c|c|c|c|c|c|}
\hline & $\begin{array}{l}\text { Number } \\
\text { of rats } \\
\text { (N) }\end{array}$ & Age (weeks) & $\begin{array}{l}\text { Body Weight } \\
\text { (grams) }\end{array}$ & $\begin{array}{l}\text { Mean Arterial Pressure } \\
(\mathrm{mmHg})\end{array}$ & $\begin{array}{l}\text { Heart Rate } \\
\quad(\mathrm{bpm})\end{array}$ \\
\hline Sham Control & 16 & $9 \pm 0.2$ & $288 \pm 18$ & $90 \pm 4$ & $336 \pm 18$ \\
\hline $\begin{array}{l}\mathrm{Nano}^{-\mathrm{TiO}_{2}} \\
200 \mu \mathrm{g}\end{array}$ & 17 & $9 \pm 0.4$ & $295 \pm 18$ & $94 \pm 4$ & $330 \pm 8$ \\
\hline $\begin{array}{c}\mathrm{Nano}^{-\mathrm{TiO}_{2}} \\
100 \mu \mathrm{g}\end{array}$ & 16 & $10 \pm 0.1$ & $319 \pm 10$ & $82 \pm 3$ & $323 \pm 10$ \\
\hline $\begin{array}{c}\mathrm{Nano}^{-\mathrm{TiO}_{2}} \\
20 \mu \mathrm{g}\end{array}$ & 12 & $9 \pm 2$ & $322 \pm 19$ & $87 \pm 3$ & $311 \pm 16$ \\
\hline
\end{tabular}

Values are mean \pm SEM; $N$, number of animals 


\section{Table 2: Arteriolar Characteristics}

\begin{tabular}{|c|c|c|c|c|c|c|c|c|c|}
\hline & $n$ & $\begin{array}{c}\text { Inner } \\
\text { diameter } \\
(\mu \mathrm{m})\end{array}$ & $\begin{array}{c}\text { Outer } \\
\text { diameter } \\
(\mu \mathrm{m})\end{array}$ & Tone (\%) & $\begin{array}{c}\text { Passive } \\
\text { diameter inner } \\
(\mu \mathrm{m})\end{array}$ & $\begin{array}{l}\text { Passive diameter } \\
\text { outer }(\mu \mathrm{m})\end{array}$ & $\begin{array}{l}\text { Wall thickness } \\
\qquad(\mu \mathrm{m})\end{array}$ & $\begin{array}{l}\text { Wall to lumen } \\
\text { ratio }\end{array}$ & $\begin{array}{l}\text { Wall Tension } \\
\text { (Newton/meter) }\end{array}$ \\
\hline $\begin{array}{l}\text { Sham } \\
\text { Control }\end{array}$ & 11 & $115 \pm 10$ & $139 \pm 17$ & $31 \pm 7$ & $184 \pm 11$ & $152 \pm 8$ & $16 \pm 3$ & $0.1 \pm 0.03$ & $0.6 \pm 0.2$ \\
\hline $\begin{array}{c}\mathrm{Nano}^{-\mathrm{TiO}_{2}} \\
200 \mu \mathrm{g}\end{array}$ & 10 & $\begin{array}{r}* \\
134 \pm 11\end{array}$ & $146 \pm 15$ & $\begin{array}{r}* \\
22 \pm 4\end{array}$ & $187 \pm 20$ & $154 \pm 19$ & $17 \pm 1$ & $0.1 \pm 0.02$ & $0.6 \pm 0.1$ \\
\hline $\begin{array}{c}\mathrm{Nano}^{-\mathrm{TiO}_{2}} \\
100 \mu \mathrm{g} \\
\end{array}$ & 9 & $128 \pm 19$ & $145 \pm 24$ & $24 \pm 1$ & $183 \pm 11$ & $149 \pm 10$ & $17 \pm 3$ & $0.1 \pm 0.02$ & $0.6 \pm 0.3$ \\
\hline $\begin{array}{c}\mathrm{Nano}^{-\mathrm{TiO}_{2}} \\
20 \mu \mathrm{g}\end{array}$ & 10 & $111 \pm 12$ & $146 \pm 12$ & $20 \pm 3$ & $180 \pm 16$ & $153 \pm 14$ & $14 \pm 2$ & $0.1 \pm 0.03$ & $0.6 \pm 0.1$ \\
\hline
\end{tabular}

Values are mean \pm SEM; $n$, number of vessels ${ }^{*} p \leq 0.05$ vs Sham Control 
Figure 1:

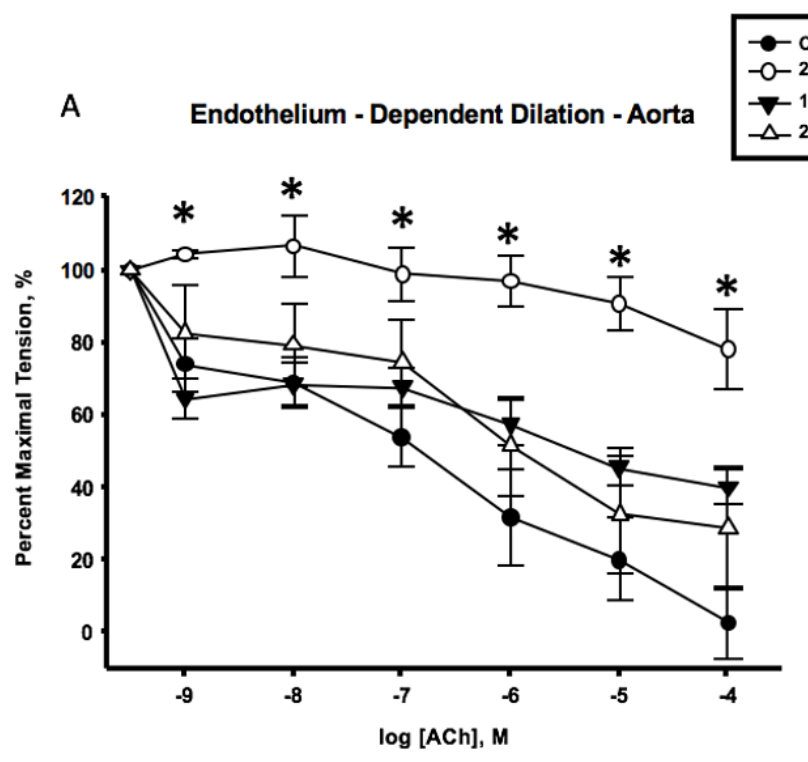

C

Endothelium - Dependent Dilation - 3rd Order MA
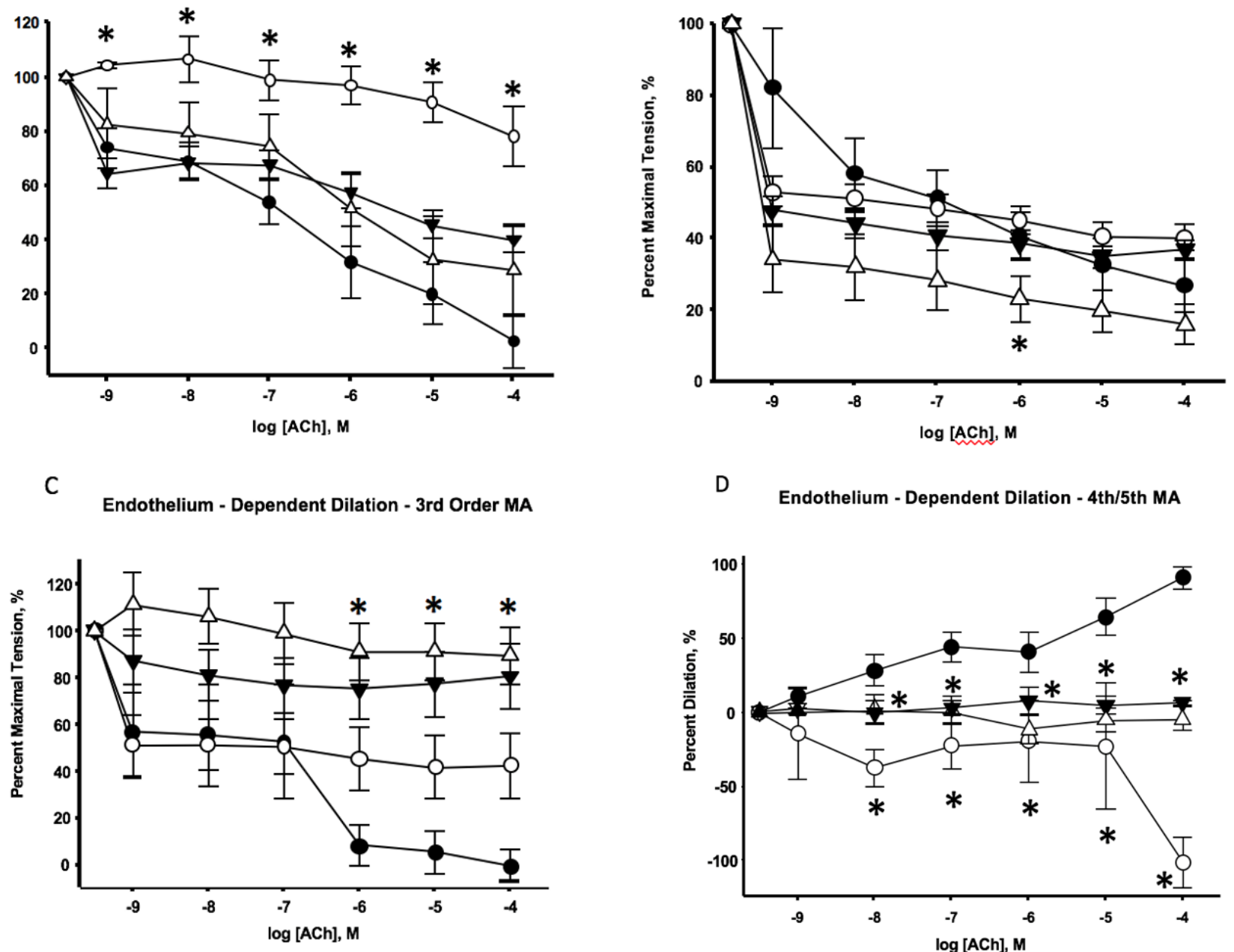

D

Endothelium - Dependent Dilation - 4th/5th MA

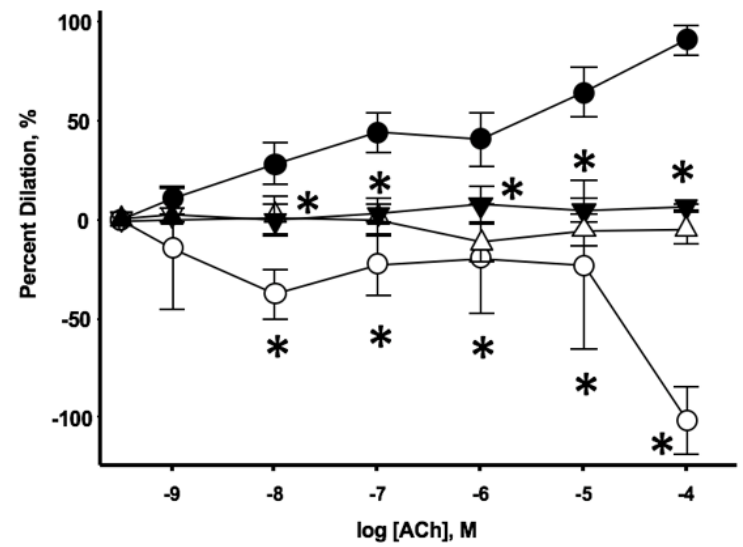


Figure 2:

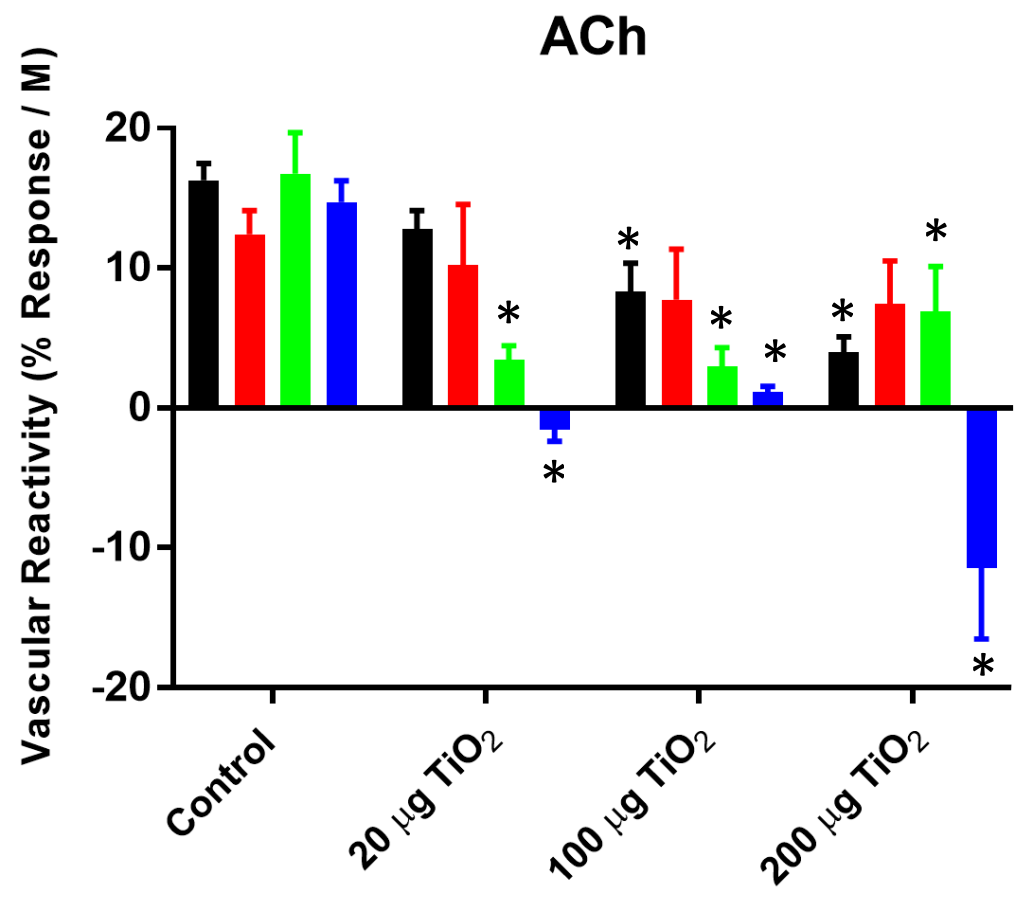

- Aorta

- Femoral Artery

- 3rd Order MA

- 4th/5th Order MA 
Figure 3:

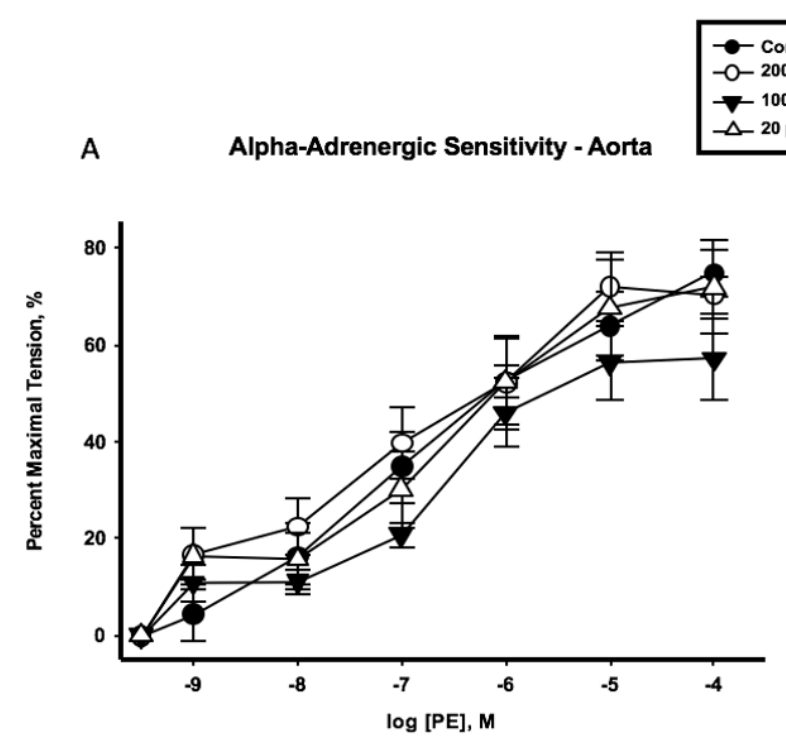

- Control

$-200 \mu \mathrm{g} \mathrm{\textrm {TiO } _ { 2 }}$

$\triangle 20 \mu \mathrm{giO}_{2}$

C Alpha-Adrenergic Sensitivity - 3rd Order MA

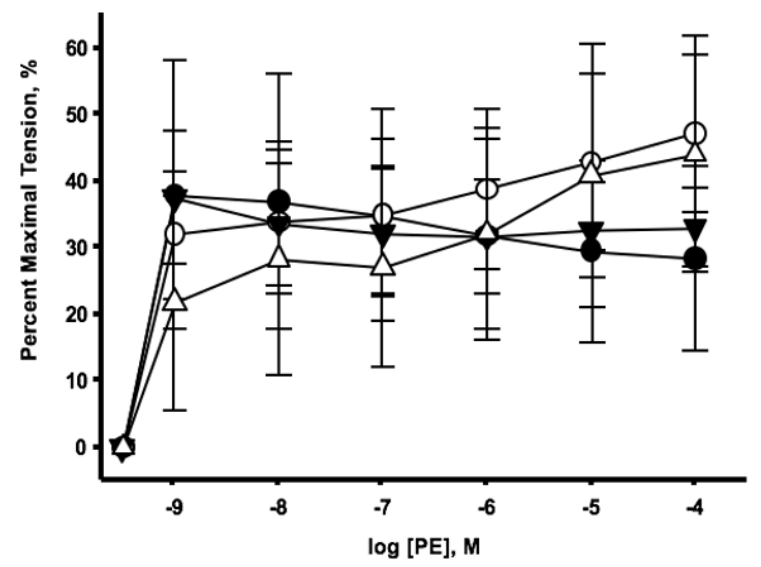

B Alpha-Adrenergic Sensitivity - Femoral Artery

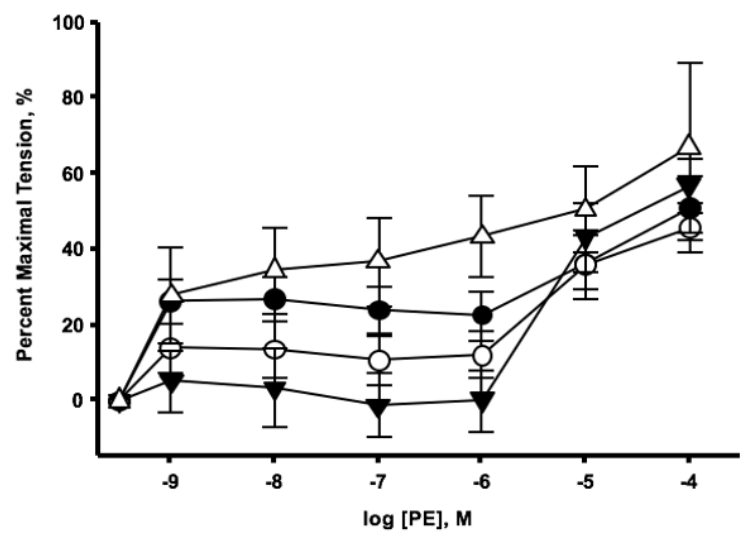

D Alpha-Adrenergic Sensitivity - 4th/5th MA

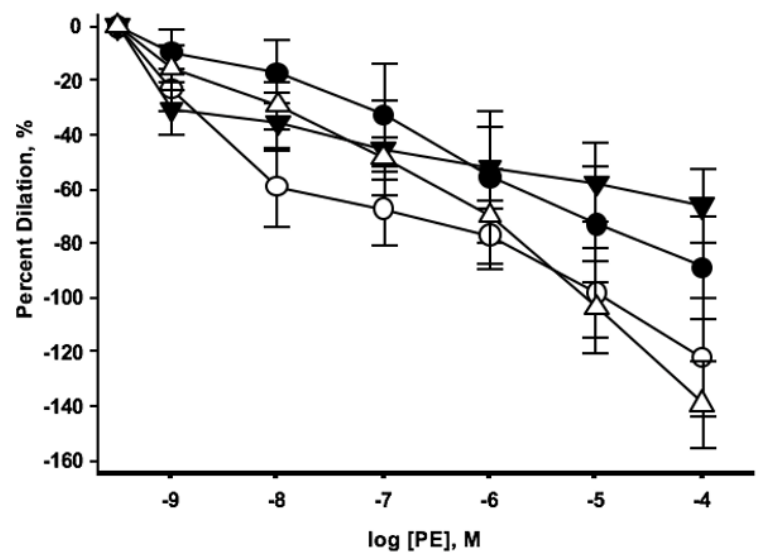


Figure 4:

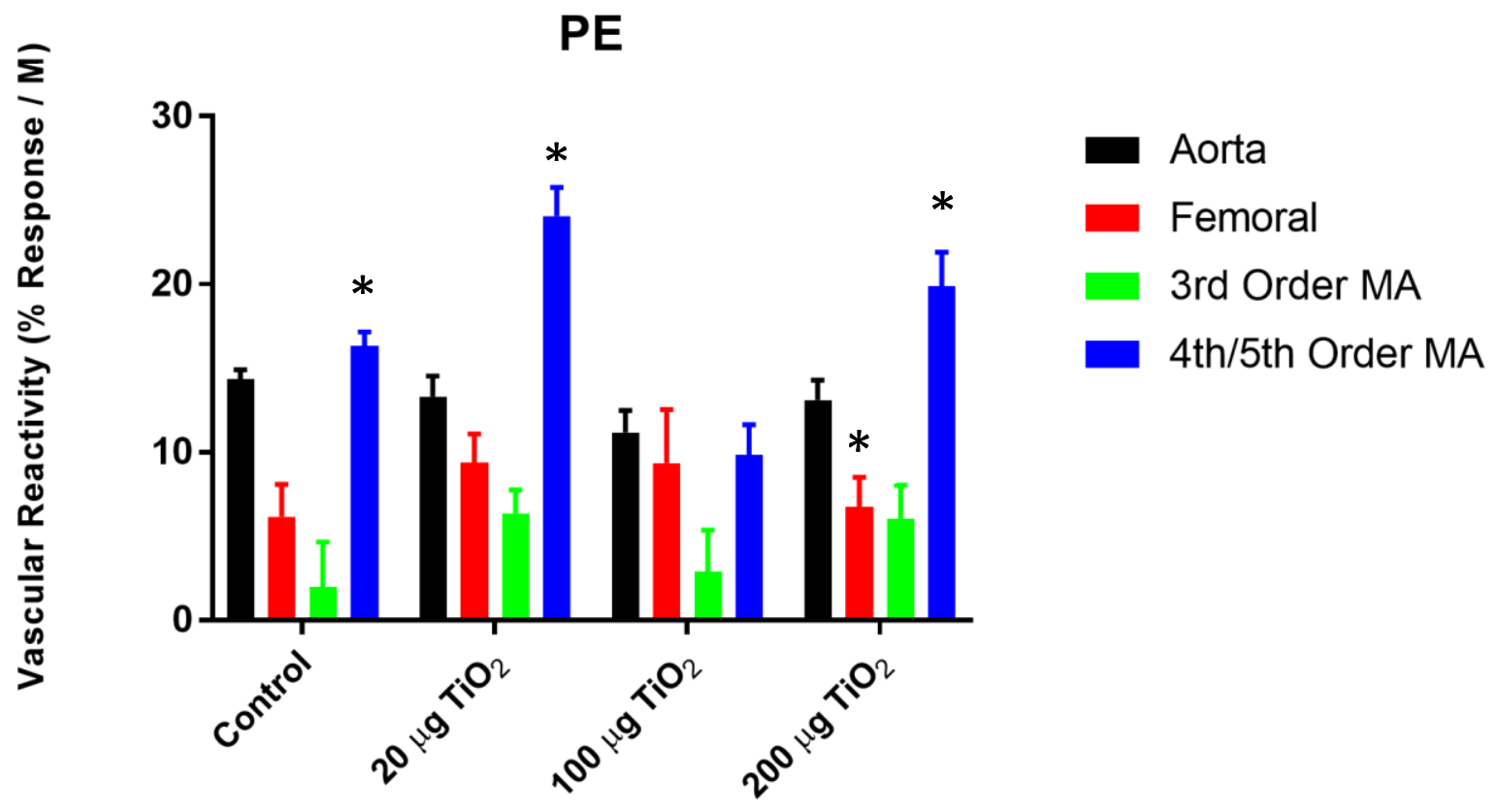


Figure 5:

A Endothelium-Independent Dilation - Aorta

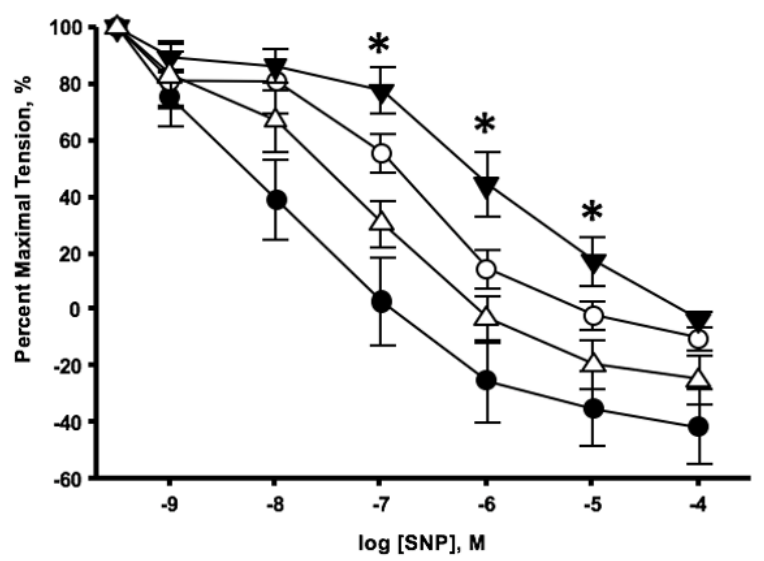

C Endothelium-Independent Dilation - 3rd Order MA

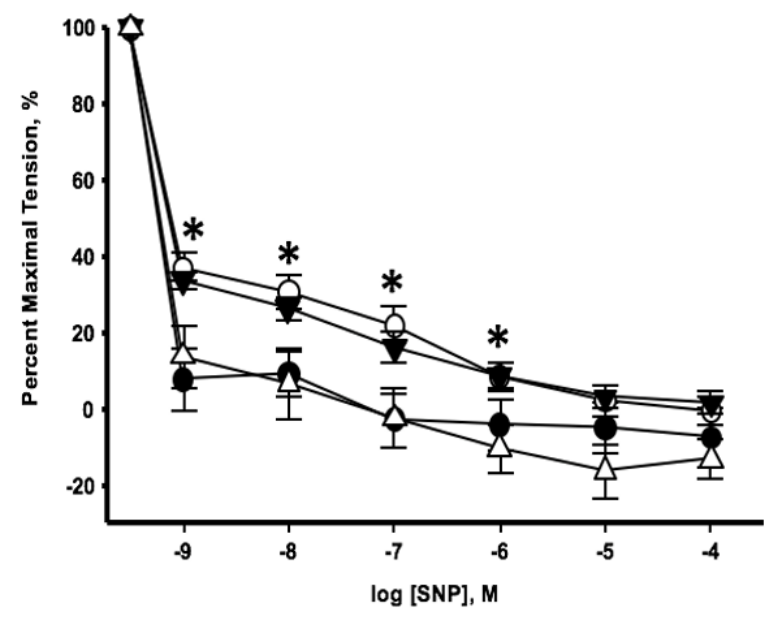

$\rightarrow-$ Control

$-\mathrm{O}-200 \mu \mathrm{g} \mathrm{\textrm {TiO } _ { 2 }}$

$\checkmark-100 \mu \mathrm{giO}_{2}$

$\triangle 20 \mu \mathrm{g} \mathrm{TiO}_{2}$
B Endothelium-Independent Dilation - Femoral Artery

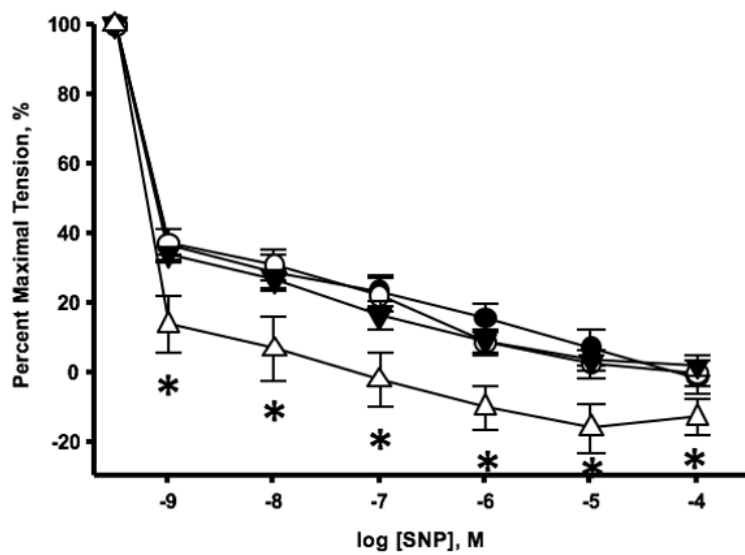

D Endothelium-Independent Dilation - 4th/5th MA

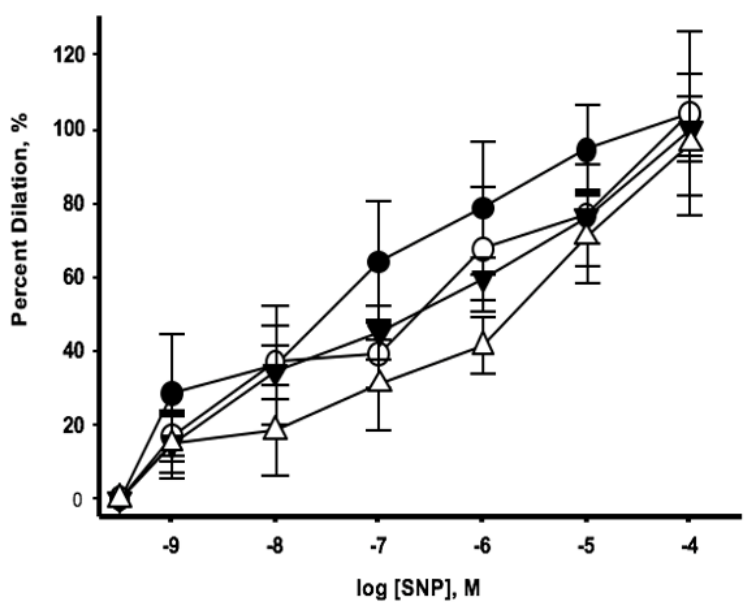


Figure 6:

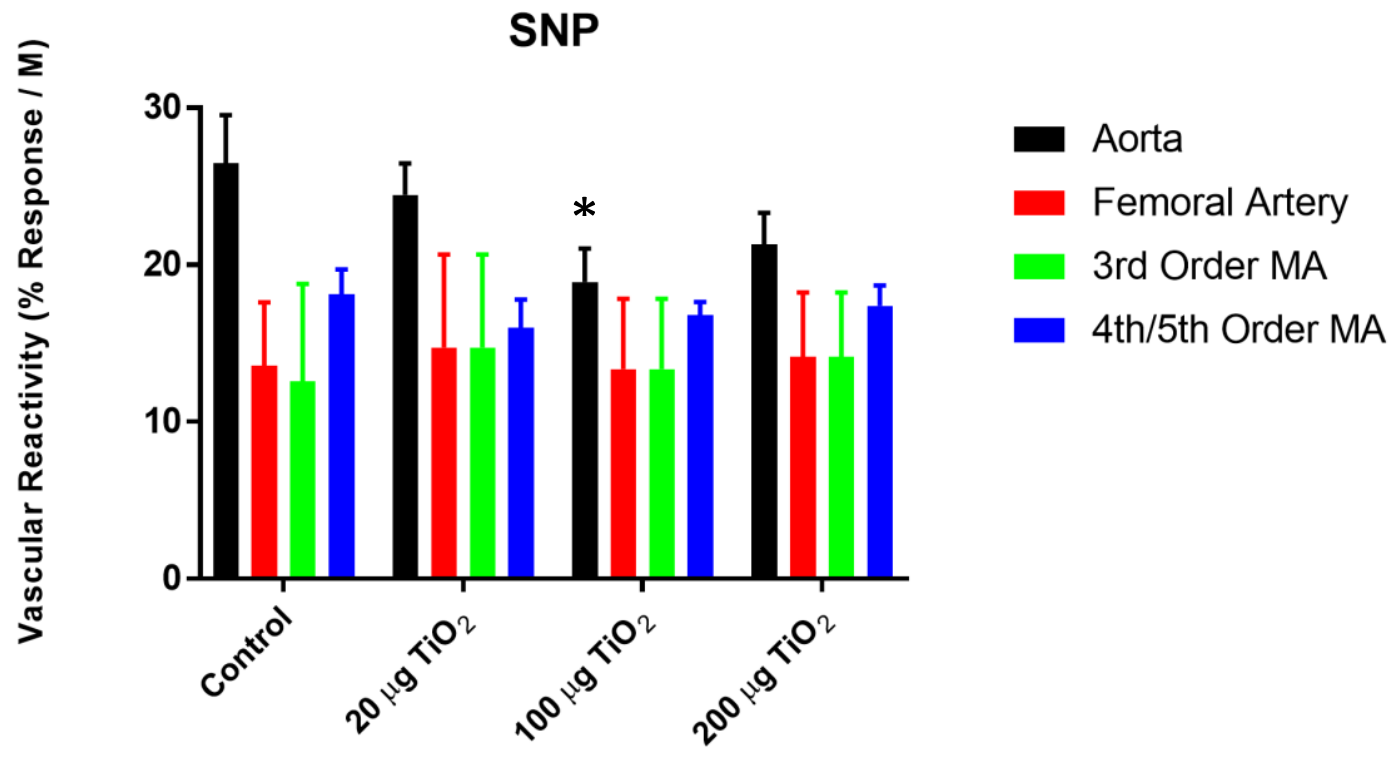




\section{Chapter 3}

Group II Innate Lymphoid Cells and Microvascular Dysfunction from Pulmonary Titanium Dioxide Nanoparticle Exposure

Running Title: Innate Lymphoid cells and Nanoparticle Exposure

Citation: Abukabda, A. B., McBride, C. R., Batchelor, T.P., Goldsmith. W.T., Bowdridge, E.C., Garner, K.L. Friend, S., \& Nurkiewicz, T. R. (2018). Group II Innate Lymphoid Cells and Microvascular Dysfunction from Pulmonary Titanium Dioxide Nanoparticle Exposure. Particle and Fiber Toxicology.

\section{Authors:}

Abukabda, A.B. ${ }^{1,2}$

McBride, C.R. ${ }^{1,2}$

Batchelor, T.P. ${ }^{1,2}$

Goldsmith, W.T.1,2

Bowdridge, E.C. ${ }^{1,2}$

Garner, K.L. ${ }^{1,2}$

Friend, $\mathrm{S}^{3}$

Nurkiewicz, T.R. ${ }^{1,2}$

\section{Affiliations:}

${ }^{1}$ Department of Physiology and Pharmacology, West Virginia University School of Medicine, Morgantown, WV, USA.

${ }^{2}$ Toxicology Working Group, West Virginia University School of Medicine, Morgantown, WV, USA.

${ }^{3}$ National Institute for Occupational Safety and Health, Morgantown, WV, USA. 


\section{Abstract:}

The cardiovascular effects of pulmonary exposure to ENM are poorly understood, and the reproductive consequences are even less understood. Inflammation remains the most frequently explored mechanism of ENM toxicity. However, the key mediators and steps between lung exposure and uterine health remain to be fully defined. The purpose of this study was to determine the uterine inflammatory and vascular effects of pulmonary exposure to nano- $\mathrm{TiO}_{2}$. We hypothesized that pulmonary nano- $\mathrm{TiO}_{2}$ exposure initiates a Th2 inflammatory response mediated by Group II innate lymphoid cells (ILC2), which may be associated with an impairment in uterine microvascular reactivity. Female, virgin, Sprague-Dawley rats (8-12 weeks) were exposed to $100 \mu \mathrm{g}$ of nano- $\mathrm{TiO}_{2}$ via intratracheal instillation 24 hours prior to microvascular assessments. Serial blood samples were obtained at 0,1,2 and 4 hours post-exposure for multiplex cytokine analysis. ILC2 numbers in the lungs were determined. ILC2s were isolated and phosphorylated nuclear factor kappa-light-chain-enhancer of activated B cells (NF-kB) levels were measured. Pressure myography was used to assess vascular reactivity of isolated radial arterioles. Pulmonary nano- $\mathrm{TiO}_{2}$ exposure was associated with an increase in IL-1ß, 4, 5 and 13 and TNF- a 4 hours post-exposure, indicative of an innate Th2 inflammatory response. ILC2 numbers were significantly increased in lungs from exposed animals $(2 \pm 0.2 \%)$ compared to controls $(0.2 \pm 0.2 \%)$. Phosphorylation of the transactivation domain (Ser-468) of NF-kB in isolated ILC2 and IL-33 in lung epithelial cells were significantly increased $(127 \pm 4 \%$ and $137 \pm$ $11 \%$ of controls respectively) by nano- $\mathrm{TiO}_{2}$ exposure. Lastly, radial endothelium-dependent arteriolar reactivity was significantly impaired ( $27 \pm 12 \%)$, while endothelium-independent dilation (7 $\pm 14 \%)$ and $\alpha$-adrenergic sensitivity $(8 \pm 2 \%)$ were not altered compared to control levels. Treatment with an anti- IL-33 antibody ( $1 \mathrm{mg} / \mathrm{kg}$ ) 30 minutes prior to nano- $\mathrm{TiO}_{2}$ exposure resulted in a significant improvement in endothelium-dependent dilation and a decreased level of IL-33 in both plasma and bronchoalveolar lavage fluid. These results provide evidence that the uterine 
microvascular dysfunction that follows pulmonary ENM exposure may be initiated via activation of lung-resident ILC2 and subsequent systemic Th2-dependent inflammation.

Keywords: engineered nanomaterials, titanium dioxide nanoparticles, microcirculation, innate lymphoid cells, inflammation 


\section{Introduction:}

Reproductive toxicity is increasingly becoming recognized as a critical aspect of ENM safety. However, the effects of ENM exposure on overall reproductive health have only recently been addressed and become a focus of intense study by numerous groups [15,16]. As with other observations of systemic biologic effects after pulmonary ENM exposures, reproductive effects are equally susceptible but poorly understood. Our group has previously reported that nano- $\mathrm{TiO}_{2}$ inhalation is associated with uterine microvascular dysfunction [43], potentially deleterious epigenomic alterations [44], and cognitive deficits in maternally exposed progeny [9]. While these studies highlight the importance of identifying the maternal effects of ENM exposure, the mechanisms linking such exposures to these negative reproductive and developmental outcomes have yet to be fully elucidated.

In the last decade, innate lymphoid cells (ILC) have emerged as a novel population of tissuespecific effector cells with the ability to initiate and regulate the innate and adaptive branches of the immune system $[25,38]$. ILCs are now divided into 3 distinct groups according to the pattern of cytokine secretion; Group 1 ILC (ILC1) are predominantly tissue-resident cells capable of secreting interferon gamma (IFN- Y) in the liver, gut, spleen, skin, peritoneum, and salivary gland [2]. The role played by ILC1 in various immunological conditions remains unclear but is currently under investigation. Group 2 ILCs (ILC2, also known as nuocytes, natural helper cells) secrete IL-4, IL-5, IL-9, and IL-13 in response to damage-associated molecular patterns or "alarmins" and have been implicated in the immune response to parasitic infections and in allergic airway inflammation [51] to several environmental and anthropogenic agents [40,52]. Lastly, group 3 ILCs (ILC3) produce IL-22 and or IL-17, are enriched at mucosal sites, contribute to the maintenance of the intestinal barriers, and may play a significant role in the promotion of the inflammatory response and etiology of inflammatory bowel diseases [48,57]. One of the most 
important alarm signals secreted by cells is interleukin-33 (IL-33) [37]. IL-33 is a member of the IL-1 family of cytokines expressed by both non-immune cells such as epithelial, endothelial, smooth muscle cells, and fibroblasts [24], as well as immune effector cells including macrophages and dendritic cells. Its role in directing the inflammatory response following pulmonary exposure to carbon nanotubes $[18,53,54]$, and ozone [26] have been previously reported. IL-33 has been demonstrated [4] to induce the release of Th2 cytokines by immune cells including ILC2s in asthmatic patients [17], and to induce airway hyperresponsiveness and increased pulmonary resistance $[6,12]$.

In view of the increasing number of studies indicating a crucial role for ILCs in the initiation of the acute inflammatory response to environmental agents, the goal of this work was to provide initial evidence of their potential involvement in the response to pulmonary ENM exposure. Therefore, the purpose of this study was threefold. First, we identified the inflammatory and uterine microvascular effects associated with acute pulmonary nano- $\mathrm{TiO}_{2}$ exposure. Second, we determined the possible role played by ILC in the immune response mounted to acute pulmonary nano- $\mathrm{TiO}_{2}$ exposure. Lastly, the microvascular and systemic effects of treatment with an IL-33 antibody (a key activator of ILC2) were determined. We hypothesized that pulmonary nano- $\mathrm{TiO}_{2}$ exposure initiates a Th2 inflammatory response mediated involving IL-33 and potentially involving ILC2, which may be associated with a systemic impairment in uterine microvascular reactivity. 


\section{Methods:}

\section{Nanomaterial Characterization}

Nano- $\mathrm{TiO}_{2}$ P25 powder, obtained from Evonik (Aeroxide $\mathrm{TiO}_{2}$, Parsippany, $\mathrm{NJ}$ ), has previously been shown to be a mixture composed primarily of anatase $(80 \%)$ and rutile $(20 \%) \mathrm{TiO}_{2}$, with a primary particle size of $21 \mathrm{~nm}$ and a surface area of $48.08 \mathrm{~m}^{2} / \mathrm{g}$ [31], and a Zeta-potential of -56.6 $\mathrm{mV}$ [29]. Elemental composition analysis of the nano- $\mathrm{TiO}_{2}$ suspension was conducted via energy dispersive spectroscopy.

Scanning electron microscopy was performed by diluting the nano- $\mathrm{TiO}_{2} 1: 100$ with filtered distilled water. $0.5 \mathrm{ml}$ of the diluted particle solution was filtered onto a $0.2 \mu \mathrm{m}$ polycarbonate filter. A wedge-shaped portion of the polycarbonate filter was mounted onto carbon double stick tape which was affixed to a $13 \mathrm{~mm}$ aluminum stub. The sample was sputter coated with gold-palladium for two minutes. The sample was imaged using a Hitachi S4800 field-emission scanning electron microscope (Tokyo, Japan).

\section{Experimental Animals and Exposure}

Female (8-10 weeks) Sprague - Dawley rats were purchased from Hilltop Laboratories (Scottdale, PA) and housed in an AAALAC approved facility at West Virginia University (WVU) with 12:12 h light - dark cycle and regulated temperature. Rats were allowed ad libitum access to food and water. All procedures were approved by the Institutional Animal Care and Use Committee of WVU. 
$100 \mu \mathrm{g}$ of nano- $\mathrm{TiO}_{2}$ were suspended in $250 \mu \mathrm{L}$ of vehicle (Normosol and $5 \%$ fetal bovine serum) for IT 24 hours prior to experimentation. Nano- $\mathrm{TiO}_{2}$ suspensions were sonicated for 1 minute to ensure homogenous distribution of nanoparticles. Rats were anesthetized using $5 \%$ isoflurane and placed on a mounting stand. $250 \mu \mathrm{L}$ of the nano- $\mathrm{TiO}_{2}$ suspension was then instilled intratracheally. Sprague - Dawley rats instilled with $250 \mu \mathrm{L}$ of vehicle were used as controls.

\section{Mean Arterial Pressure (MAP) Acquisition}

Rats were anesthetized with isoflurane gas (5\% induction, $2-3.5 \%$ maintenance). The animals were placed on a heating pad to maintain a $37^{\circ} \mathrm{C}$ rectal temperature. The trachea was intubated to ensure an open airway and the right carotid artery was cannulated to acquire mean arterial pressure (MAP). The MAP was measured via a pressure transducer and recorded by PowerLab830 (AD Instruments, Colorado Springs, CO).

\section{Multiplex Cytokine Panels of Serum and Bronchoalveolar Lavage Fluid}

Whole blood (1-2 ml) was collected via tail vein puncture from exposed and control animals at time 0, 1 hour, 2 hours, and 4 hours after exposure into EDTA vacutainers and centrifuged (1000 x g) to collect plasma which was flash-frozen in liquid nitrogen and stored at $-80{ }^{\circ} \mathrm{C}$ until analysis. Rats were euthanized, and tissues harvested for further analysis. Multi-spot inflammatory assays were completed per manufacturer's directions (Meso Scale Diagnostics, Rockville, MD) for:

lipocalin-2, TSP-1, TIMP-1, MCP-1, interferon (IFN-y), interleukin (IL)-1ß, IL-4, IL-5, IL-6, KC/GRO, IL-10, IL-13, and TNF- $\alpha$. 


\section{Immunohistochemistry}

Gelatin-coated cover slips of serial tissue sections were incubated with anti-rabbit (1:100) IL-33 (Cloud-Clone Corp., Katy, TX) overnight at $4{ }^{\circ} \mathrm{C}$, followed by 3 five-minute washes with cold PBS with $0.1 \%$ Triton-X. The sections were then incubated with a FITC conjugated goat anti-rabbit ( $1: 100$, Invitrogen, Carlsbad, CA) at $37^{\circ} \mathrm{C}$ for one hour, followed by 3 five-minute washes with cold PBS with $0.1 \%$ Triton-X. Cover slips were then mounted on slides for visualization on a Zeiss fluorescent microscope (Zeiss, Thornwood, NY).

\section{Preparation of Cell Suspensions}

Lungs were perfused with sterile PBS by injection into the right ventricle to remove remaining blood and then placed in PBS containing $20 \mathrm{mg} / \mathrm{ml}$ Collagenase A, $2.4 \mathrm{U} / \mathrm{ml}$ Dispase II solution, and $50 \mu \mathrm{l} / \mathrm{ml}$ DNAse (Roche, Indianapolis, IN) for 30 minutes.

The lung tissue was dissociated using the gentleMACS Octo Dissociator (Miltenyi Biotec, Auburn, CA) and then centrifuged (1100 x g for 10 minutes) to collect the sample material. The sample was resuspended with PBS and used for magnetic cell separation and flow cytometry.

\section{Flow Cytometry}

\section{Antibodies and reagents}

ILC1 and ILC2 numbers 4 hours after exposure to nano- $\mathrm{TiO}_{2}$ from lung tissue were determined by flow cytometry as previously described [8]. Briefly, ILC1 were defined as LinCD45+CD161+CD335+, while ILC2 were defined as Lin-CD45+CD90.1+KLRG$1+\mathrm{CD} 25+\mathrm{IL} 1 \mathrm{R} 1+$. 
Monoclonal antibodies specific for CD90.1 (FITC), KLRG1 (APC EFLUOR 780), CD25 (APC), CD161 (PERCP EFLUOR 710), and CD335 (EFLUOR 450) were purchased from eBioscience (San Diego, CA). Lineage cocktail (Lin; ALEXA FLUOR 700) was used to gate out (CD3, CD14, CD16, CD19, CD20, and CD56: Biorad, Hercules, CA) CD3 T lymphocytes, CD14 Monocytes, CD16 NK cells, granulocytes, CD19 B lymphocytes, CD20 B lymphocytes, and CD56 NK cells. Antibodies for CD45 (Cyanine 5) were from Invitrogen (Carlsbad, CA), while those for IL1R1 (PE) where from Sino Biologicals (North Wales, PA). Flow cytometry was performed on a FACSAria (BD Bioscience, Franklin Lakes, NJ). Data were analyzed with FCS Express 6 Software (De Novo Software, Glendale, CA).

\section{Isolation of Group II Innate Lymphoid Cells}

ILC2 were isolated as per manufacturer's instructions (Miltenyi Biotec, Auburn, CA). Briefly, lineage-positive cells were indirectly labeled with a cocktail of ALEXA FLUOR 700-conjugated antibodies, as primary labeling reagent, and antibodies conjugated to MicroBeads were used as secondary labeling reagents. In the second step, lineage-negative cells were labeled with CD45, CD90.1, KLRG-1, CD25, and IL1R1, labeled with MicroBeads and isolated by positive selection from the pre-enriched lineage negative cell-fraction by separation over a MACS Column (Miltenyi Biotec, Auburn, CA), which was placed in the magnetic field of a MACS Separator. After negative selection, the cells were subsequently eluted as the positively selected cell fraction containing ILC2.

\section{Cytokine Analysis of Cell Culture Media and Measurement of Phospho-NF-kB}

Isolated ILC2s were incubated $\left(37^{\circ} \mathrm{C}, 90 \%\right.$ humidity, $\left.5 \% \mathrm{CO}_{2}\right)$ overnight in supplemented $(10 \%$ fetal bovine serum, $1 \%$ penicillin/streptomycin, $1 \%$ sodium pyruvate, $1 \%$ L-glutamine) Dulbecco`s 
Modified Eagle Medium (Corning, Manassas, VA). The following day, the cell culture medium and cellular portion were separated via centrifugation (1100 $\mathrm{xg}$ for 10 minutes). IL-4, IL-5, and IL-13 levels were measured in the cell culture medium via multi-spot inflammatory assays per manufacturer's directions (Meso Scale Diagnostics, Rockville, MD). Lastly, isolated cells were lysed and phospho-NF-kB (Ser468 and Ser536) was measured (Meso Scale Diagnostics, Rockville, MD).

\section{Systemic Treatment with anti-Interleukin-33 Antibody}

Rats were treated 1 hour prior to pulmonary nano- $\mathrm{TiO}_{2}$ exposure with pharmacological grade polyclonal anti-IL-33 antibody (CAT\#ABF108, EMD Millipore, Temecula, CA: intraperitoneal $1 \mathrm{mg} / \mathrm{kg}$ ). The antibody and dosage were selected based on previous murine studies [22]. The antibody used was a polyclonal anti-IL-33 antibody developed in the rabbit with specific reactivity toward mouse IL-33 and expected cross reactivity due to the close homology to rat IL-33. We determined the interspecies homology of the anti-IL-33 target between rats and mice to be $87 \%$ (Supplementary Figure 1). Plasma, bronchoalveolar lavage fluid, and lung tissue were obtained 4 hours post-exposure from treated animals for measurement of IL-33. Uterine microvascular assessments were conducted 24 hours after treatment in a separate cohort of animals.

\section{Pressure Myography Microvessel Preparation}

After measuring MAP, the uterus was removed and placed in a dissecting dish with physiological salt solution (PSS) maintained at $4{ }^{\circ} \mathrm{C}$. Radial arterioles were isolated, transferred to a vessel chamber, cannulated between two glass pipettes, and tied with silk sutures in the chamber (Living Systems Instrumentation, Burlington, VT). The chamber was superfused with fresh oxygenated $\left(5 \% \mathrm{CO}_{2} / 21 \% \mathrm{O}_{2}\right)$ PSS and warmed to $37^{\circ} \mathrm{C}$. Arterioles were pressurized to $60 \mathrm{~mm} \mathrm{Hg}$ using a 
servo control system and extended to their in situ length. Internal and external arteriolar diameters were measured using video calipers (Colorado Video, Boulder, CO).

\section{Arteriolar Reactivity}

Previous work by our group has shown that ENM exposure impacts endothelium-dependent dilation most severely within 24 hours, a condition which has been shown to improve but did not fully return to control levels after 168 hours. Based on these findings, all vascular assessments for this study were conducted 24 hours post-exposure [42].

Arterioles were allowed to develop spontaneous tone, defined as the degree of constriction experienced by a blood vessel relative to its maximally dilated state. Vascular tone ranges from $0 \%$ (maximally dilated) to $100 \%$ (maximal constriction). Vessels with a spontaneous tone $\geq 20 \%$ less than initial tone were included in this study. After equilibration, various parameters of arteriolar function were analyzed. Vessels that did not develop sufficient spontaneous tone were not included in the data analysis.

Endothelium-Dependent Dilation - arterioles were exposed to increasing concentrations of acetyl choline (ACh: $10^{-9}-10^{-4} \mathrm{M}$ ) added to the vessel chamber.

Endothelium-Independent Dilation-increasing concentrations of sodium nitroprusside (SNP: $\left.10^{-9}-10^{-4} \mathrm{M}\right)$ were used to assess arteriolar smooth muscle responsiveness.

Arteriolar Vasoconstriction-arterioles were exposed to increasing concentrations of phenylephrine (PE: $\left.10^{-9}-10^{-4} \mathrm{M}\right)$. The steady state diameter of the vessel was recorded for at least 2 min after each dose. After each dose curve was completed, the vessel chamber was 
washed to remove excess chemicals by carefully removing the superfusate and replacing it with fresh warmed oxygenated PSS. After all experimental treatments were complete, the PSS was replaced with $\mathrm{Ca}^{2+}$-free PSS until maximum passive diameter was established.

\section{Pressure Myography Calculations}

Data are expressed as means \pm standard error. Spontaneous tone was calculated by the following equation:

$$
\text { Spontaneous tone }(\%)=\left\{\frac{(D m-D i)}{D i}\right\} \times 100
$$

, where $\mathrm{Dm}$ is the maximal diameter and $\mathrm{Di}$ is the initial steady state diameter recorded prior to the experiment. Active responses to pressure were normalized to the maximal diameter using the following formula:

$$
\text { Normalized diameter }=\text { Dss } / D m
$$

, where Dss is the steady state diameter recorded during each pressure change. The experimental responses to ACh, PE, and SNP are expressed using the following equation:

$$
\text { Diameter }(\text { percent maximal diameter })=\left\{\frac{(\text { Dss }- \text { Dcon })}{(D m-D c o n)}\right\} \times 100
$$

, where DCon is the control diameter recorded prior to the dose curve, DSS is the steady state diameter at each dose of the curve. The experimental response to PE is expressed using the following equation:

$$
\text { Diameter }(\text { percent maximal diameter })=\left\{\frac{(\text { Dcon }- \text { Dss })}{(\text { Dcon })}\right\} \times 100
$$


Wall thickness (WT) was calculated from the measurement of both inner (ID) and outer (OD) steady state arteriolar diameters at the end of the $\mathrm{Ca}^{2+}$ free wash using the following equation:

$$
W T=(O D-I D) / 2
$$

Wall-to-lumen ratio (WLR) was calculated using the following equation:

$$
W L R=W T / I D
$$

\section{Statistics}

Point-to-point differences in the dose response curves were evaluated using two-way repeated measures analysis of variance (ANOVA) with a Tukey`s post-hoc analysis when significance was found. The slopes of the dose response curves were determined through a nonlinear regression. The animal characteristics, vessel characteristics and dose response curve slopes were analyzed using a one-way ANOVA with a Tukey post-hoc analysis when significance was found. All statistical analysis was completed with GraphPad Prism 5 (San Diego, CA) and SigmaPlot 11.0 (San Jose, CA). Significance was set at $p<0.05, n$ is the number of arterioles, and $N$ is the number of animals. 


\section{Results:}

\section{SEM Images and Mass Spectrometry of Nano- $\mathrm{TiO}_{2}$}

Figure 1 shows field-emission scanning electron microscope images (Hitachi S4800, Tokyo, Japan) of the nano- $\mathrm{TiO}_{2}$ suspension used for this study. As seen in Figure $1 \mathbf{A}$, the suspended particles present significant agglomeration. Figure 1 B shows the elemental composition of the nano- $\mathrm{TiO}_{2}$ suspension (Bruker, Billerica, MA), indicating the prevalent presence of titanium.

\section{Animal and Vessel Characteristics}

No significant changes were observed in age, mean arterial pressure (MAP), heart rate and body weight between control and exposure groups (Table 1). Additionally, radial arteriolar inner and outer diameter, tone, passive diameter, wall thickness, wall to lumen ratio and calculated wall tension were not affected by nano- $\mathrm{TiO}_{2}$ exposure and treatment with an anti-IL-33 antibody (Table 2). From these results we can infer that acute nano- $\mathrm{TiO}_{2}$ exposure does not alter vascular tone or the balance of its contributing influences.

\section{Acute Plasma and BALF Cytokine Secretion Patterns Following Pulmonary Nano-TiO 2 Exposure}

A time-course study was initially performed to identify the temporal effect of nano- $\mathrm{TiO}_{2}$ on cytokine secretion. Plasma samples were obtained at $0,1,2$, and 4 hours following nano- $\mathrm{TiO}_{2}$ exposure by tail vein puncture. Figure 2 shows the results of the multiplex analysis. No differences existed at time 0,1 or 2 hours post-exposure. However, significant differences were detected 4 hours postexposure in plasma levels of the pro-inflammatory cytokines IL-4, IL-5, IL-13, TNF- $\alpha$, IL-1 $\beta$, while 
no alterations in the cytokines IFN- $\gamma, \mathrm{KC} / \mathrm{GRO}, \mathrm{TNF}-\alpha, \mathrm{IL}-10$, IL-6, MCP-1, TIMP-1, Lipocalin-2, and TSP-1 were noted (Data not shown).

In order to confirm the results observed in the plasma, the cytokine levels in the BALF were also measured 4 hours post-exposure. Multiplex analysis of the BALF (Figure 3) also showed an almost 5-fold increase in IL-5 and a 6-fold increase in IL-4, IL-13, TNF- $\alpha$, and IL-1 $\beta$, while no changes were seen in the other cytokines (Data not shown). Based on the collective cytokine profile associated with this exposure paradigm, the results provide evidence that acute pulmonary nano- $\mathrm{TiO}_{2}$ exposure may trigger a T-Helper cell type 2 (Th2) response beginning 4 hours postexposure.

\section{IL-33 Secretion by Lung Epithelial Cells Pre- and Post- Exposure and Following Treatment with an anti-IL-33 Antibody}

IL-33 plays a significant role in initiating and modulating lung inflammatory and immunological responses [19], by inducing the secretion of Th2 pro-inflammatory cytokines. It is constitutively present in mucosal epithelial cells and acts as a "danger" signal after tissue injury by activating immune cells. To determine the effect of nano- $\mathrm{TiO}_{2}$ on pulmonary and plasma IL-33 levels, immunohistochemistry of tracheal sections was conducted 4 hours post-exposure. In a separate cohort of control and exposed animals, multiplex analysis of plasma and BALF IL-33 was also performed 4 hours post-exposure. Figure 4 shows representative images of lung sections from control and exposed rats stained for IL-33 4 hours post-exposure and the quantitative measurements for both groups. Relative fluorescence intensity of IL-33 was significantly increased in exposed animals by $26.8 \pm 4.3 \%$, while plasma and BALF levels indicated a $38 \pm 2 \%$ and $171 \pm 13 \%$ increase in IL-33 respectively (Figure 5). Interestingly, treatment 1 hour prior to 
exposure to nano- $\mathrm{TiO}_{2}$ with pharmacological grade polyclonal anti-IL-33 antibody (EMD Millipore, Temecula, CA: intraperitoneal $1 \mathrm{mg} / \mathrm{kg}$ ) resulted in a decrease in plasma and BALF IL-33 levels 4 hours post-exposure ( $61 \pm 2 \%$ in plasma and $150 \pm 7 \%$ in BALF) when compared to nano- $\mathrm{TiO}_{2}$ exposed and untreated animals. The results herein described provide evidence that reveals the critical role played by IL-33 in the initiation of the inflammatory response to ENM.

\section{Flow Cytometric Analysis of ILC1 and ILC2}

Polarization of T-cells requires several days [21], therefore, the early appearance of Th2 cytokines in the circulation after nano- $\mathrm{TiO}_{2}$ suggests that other, more rapid immune effector cells may be involved. Previous work has indicated that both ILC1 and ILC2 $[11,14]$ reside in the lungs and may be involved in lung inflammatory responses and pathology. Therefore, we next wanted to determine the effect of nano- $\mathrm{TiO}_{2}$ exposure on ILC1 and ILC2 levels in the lungs. For this reason, multi-parametric flow cytometric analysis was conducted on lung tissue from control and nano$\mathrm{TiO}_{2}$ exposed Sprague-Dawley rats for ILC1 (Data not shown) and ILC2 (Figure 6) 4 hours postexposure. No significant differences were noted in ILC1 levels pre- and post- exposure while ILC2 levels increased from $0.2 \pm 0.2 \%$ to $2 \pm 0.2 \%$ (Figure 7 A and B).

\section{NF-kB Phosphorylation and Th2 Cytokine Secretion by Isolated ILC2}

Activation of the NF-KB pathway has been shown to play a role in inflammation through its ability to induce the transcription of proinflammatory genes $[33,34]$. Specifically, phosphorylation of NF$\mathrm{KB}$ at both serine residues 468 and 536 are associated with inflammatory response [33,34]. Therefore, levels of phospho- NF- $\mathrm{kB}$ were measured in isolated ILC2 from control and nano$\mathrm{TiO}_{2}$ exposed animals (Figure 8). No significant differences were noted in phosphorylation of the Serine-536 residue between the 2 groups, while phosphorylation of Serine-468 was increased after acute pulmonary nano- $\mathrm{TiO}_{2}$ exposure ( $129 \pm 7 \%$ of control levels). 
Lastly, isolated ILC2 were cultured overnight and IL-4, IL-5 and IL-13 levels in the cell-culture media were measured. Nano- $\mathrm{TiO}_{2}$ resulted in a significant increase in IL-4, IL-5, and IL-13 (122 $\pm 7 \%, 141 \pm 9 \%, 159 \pm 8 \%$ of control levels respectively) (Figure 9).

\section{Effect of Acute Pulmonary Nano- $\mathrm{TiO}_{2}$ Exposure and Systemic Immunotherapy on Uterine Radial Arterioles}

\section{Endothelium-Dependent Dilation}

In order to identify the acute reproductive effects associated with nano- $\mathrm{TiO}_{2}$ exposure, radial arterioles were isolated from the uterus of control and exposed animals 24 hours post-exposure and endothelium-dependent and independent dilation along with adrenergic sensitivity were tested. Previous work by our group has shown that ENM exposure impacts vascular reactivity most severely within 24 hours, a condition which has been shown to improve but not fully return to control levels after 168 hours [42]. Based on these findings, all vascular assessments for this study were conducted 24 hours post-exposure.

Endothelium-dependent dilation of radial arterioles was significantly impaired following intratracheal instillation of $100 \mu \mathrm{g}$ of nano- $\mathrm{TiO}_{2}$ (Figure 10), with a mean decrease in dilation of $49 \pm 7 \%$ compared to controls and $24 \pm 10 \%$ compared to anti-IL-33 antibody-treated rats. These results suggest that pulmonary nano- $\mathrm{TiO}_{2}$ exposure disrupts normal physiological vascular endothelial function and that pre-treatment with an anti-IL-33 antibody partially attenuates the systemic acute inflammatory cascade triggered by lung-derived IL-33. 
In contrast to endothelium-dependent relaxation, no point to point differences were seen in $\alpha$ adrenergic and endothelium-independent dilation between control, nano- $\mathrm{TiO}_{2}$ exposed, and treated groups (Data not shown). 


\section{Discussion:}

The inflammatory paradigm tested herein has been suggested by previous studies $[18,36,58]$. While these studies explored pulmonary responses to exogenously administered IL-33, carbon nanotubes and viral infection, our findings provide additional evidence of a similar link between acute pulmonary ENM exposure and subsequent inflammatory mechanisms with systemic microvascular consequences. Perhaps the most important finding of this study is the identification of IL-33 as a potential contributor to the systemic inflammatory phenotype and microvascular dysfunction resulting from acute pulmonary ENM exposure.

We hypothesized that the innate response to pulmonary nano- $\mathrm{TiO}_{2}$ exposure is triggered by the secretion of IL-33 by lung epithelial cells. IL-33, a member of the IL-1 family, plays a significant role in initiating and modulating lung inflammatory and immunological responses [27], by inducing the secretion of Th2 pro-inflammatory cytokines through the Suppression of Tumorigenicity 2 (ST2) receptor in a variety of immune cells [59]. Previous studies have shown that exposure to multi-walled carbon nanotubes triggers the IL-33/ST2 axis leading to the activation of mast-cells and macrophages [4]. Interestingly, IL-33 is also required for the maturation, activation and egress of ILC2 lineage cells from the bone marrow to specific target organs [46]. Several previous studies by our group and others have extensively investigated the cardiovascular effects of pulmonary ENM exposure $[1,30]$. These studies consistently reveal that nano- $\mathrm{TiO}_{2}$ exposure increases leukocyte activation [38], proinflammatory mediators [41], and oxidative stress [20]. Collectively, this results in endothelium-dependent dysfunction. ILCs are a recently identified subpopulation of immune effector cells $[27,39]$. They are critical, non-cytolytic tissue-resident cells that can be activated and respond acutely to danger signals or "alarmins" such as IL-33 from mucosal tissue by producing a distinctive array of cytokines to maintain mucosal integrity. Group II ILCs specifically, secrete the cytokines IL-5, IL-13, IL-9, amphiregulin, and low quantities of IL-4 [36] 
and have been linked to several lung-associated conditions including pathogen infections (viruses and helminths), asthma, and pulmonary fibrosis [13]. Lung ILC2 have an important role in regulating tissue remodeling and repair during acute epithelial injury and asthma [28]. Considered together, it is reasonable to speculate that the IL-33/ILC2 axis may impact systemic microvascular function after pulmonary ENM exposure.

Perhaps, the most significant finding of this study is the identification of a potential role for IL-33 in the initiation of an acute inflammatory response and the effects on systemic microvascular dysfunction (Figure 10). This was achieved by interrupting the IL-33/ST2 signaling axis with an anti-IL-33 antibody treatment prior to ENM exposure. In these experiments, the control group (IT saline) did not receive the anti-IL-33 antibody. The scientific rationale for this decision was that the technique (isolated, perfused and pressurized arterioles) used to assess microvascular dysfunction is a plasma free system. As such, it was impossible to assess the role of the complete IL-33/ST2 axis on arteriolar tone and/or reactivity. Whereas, this approach directly measured the impact that IL-33 inflammatory signaling has on microvascular function. It remains a distinct possibility that the IL-33/ST2 axis activated by pulmonary ENM exposure directly influences microvascular tone and/or reactivity. IL-33 expression has been identified in human vascular endothelial cells; whereas, is absent in murine vascular tissue [32]. This supports our postulate that IL-33 is impacting microvascular function via inflammation, rather than a direct activation of the ST2 receptor. Future in vivo studies must directly assess the impact of circulating IL-33 on microvascular tone, reactivity and blood flow.

While our study provides plausible mechanistic insights into both the inflammatory and microvascular effects associated with ENM exposure, additional considerations as well as the 
limitations must be kept in mind. ENM exposure has been shown to affect other innate immune effector cells such as neutrophils [35], macrophages [5,48], and dendritic cells [23]. Therefore, other mechanisms including local generation of reactive of oxygen species, secretion of chemokines and prostaglandins by macrophages and stimulation of the adaptive immune system by antigen-presenting cells may play a role in the systemic inflammatory response and vascular effects seen in this study. It is also worth noting that in this study an immune response associated with pulmonary ENM exposure was observed as early as 4 hours post-exposure, as evidenced not only by an increase in the proportion of lung-resident ILC2s (Figure 7), but also by augmented levels of the Th2 cytokines IL-4, IL-5, and IL-13 (Figures 2 and 3). This response timeframe is in agreement with previous studies, that have reported IL33, ILC2 numbers as well as lung and serum Th2 cytokines increase within $6-12$ hours of lung injury $[50,51]$. Furthermore, isolated ILC2s have been shown to respond dose-dependently to transient or continuous stimulation with IL-2 and/or IL-33 within 3 hours [52]. Similarly, nano- $\mathrm{TiO}_{2}$ instillation at lung burdens of 40-160 $\mu \mathrm{g} / \mathrm{rat}$ has also been shown to increase BALF inflammatory mediators within 4 hours of exposure [53]. We report herein a similar phenomenon as the lung burden used in this study was comparable at $100 \mu \mathrm{g} / \mathrm{rat}$.

Our findings contribute consistent evidence for a role of ILC2s in the acute pulmonary response to ENM exposure. Due to the inherent limitations associated with our experimental in vivo model, we were unable to directly establish causality. In order to directly confirm such an observation, ILC2 deletion would be necessary. Our research program has a long-standing interest in the systemic microvascular consequences of pulmonary particle exposure. Rat models are primarily used in our in vivo studies as they are the most appropriate and widely used animals for microvascular research. However, they are not commonly or easily adapted for transgenic studies. As such, an ILC2 rat deletion model does not exist. If such a model were created in the 
future, it would then be possible to directly confirm a causative relationship between ENM exposure and ILC2 activation, proliferation and recruitment.

Lastly, to allow periodic blood sampling for cytokine analysis, intratracheal instillation was chosen as the preferred exposure method. The above-mentioned dose of nano- $\mathrm{TiO}_{2}$ was selected based on previous studies conducted by our group which have shown that it is associated with significant microvascular impairment, oxidative and nitrosative stress and alveolar macrophage recruitment $[36,54]$. Additionally, to better understand the relevance of the exposure paradigm used in this study to humans, alveolar surface areas must be known [47]. The rat alveolar surface area is 0.4 $\mathrm{m}^{2} /$ lung. Therefore, the rat burden of $50 \mu \mathrm{g} / \mathrm{lung}$ would result in $125 \mu \mathrm{g} / \mathrm{m}^{2}$. Given that the human alveolar surface area is $102 \mathrm{~m}^{2}$, the equivalent human burden of this exposure paradigm would be $12.75 \mathrm{mg}$. The next logical question is: How long would it take to achieve this burden in humans? In this regard, lung burden may be calculated as:

nano- $\mathrm{TiO}_{2}$ aerosol concentration · minute ventilation · exposure duration · deposition fraction, with the following values:

$25.5 \mathrm{mg}=$ nano $-\mathrm{TiO}_{2}$ aerosol concentration $\cdot 7600 \mathrm{ml} / \mathrm{min} \cdot(8 \mathrm{hr} /$ day $\cdot 60 \mathrm{~min} / \mathrm{hr}) \cdot 14 \%$, and therefore:

$$
25.5 \mathrm{mg}=\text { nano }-\mathrm{TiO}_{2} \text { aerosol concentration } \cdot 0.5 \mathrm{~m}^{3} / \text { day } .
$$

Considering both the National Institute for Occupational Safety and Health (NIOSH) and the Occupational Safety and Health Administration (OSHA) Permissible Exposure Limit $\left(0.3 \mathrm{mg} / \mathrm{m}^{3}\right.$ and $5 \mathrm{mg} / \mathrm{m}^{3}$ respectively) [43], it would require 0.34 working years or 122 working days for a human to achieve similar exposure levels to those used in this study. 
In conclusion, the current study provides novel evidence that links acute pulmonary ENM exposure and systemic microvascular effects. Because this inflammatory link exists between the lung and the uterine microcirculation, it remains to be determined if such an axis extends to the placenta and or fetus. 
Funding information: We would like to acknowledge our financial support: R01-ES015022 (TRN), and NSF-1003907 (TRN and ABA).

Acknowledgements: We thank Kevin Engels from the West Virginia University (WVU) Department of Physiology and Pharmacology for his technical assistance in this study, and Dr. Kathleen Brundage from the Flow Cytometry \& Single Cell Core Facility at WVU (FORTESSA S10 OD016165, FACSAria S10 RR020866).

Disclaimer: The findings and conclusions in this report are those of the author(s) and do not necessarily represent the official position of the National Institute for Occupational Safety and Health, Centers for Disease Control and Prevention. 
Figure Legends:

Figure 1. Characterization of $\mathrm{Nano}^{-\mathrm{TiO}_{2}}$. (A) SEM image and (B) Energy dispersive spectroscopy showing elemental composition of the nano- $\mathrm{TiO}_{2}$ suspension used in this study.

Figure 2. Nano- $\mathrm{TiO}_{2}$ exposure increases $\mathrm{T}$-Helper type II cytokines 4 hours post-exposure. Multiplex cytokine analysis showing concentrations of (A) IL-4, (B) IL-1 $\beta$, (C) TNF- $\alpha$, (D) IL-5, and (E) IL-13. Serum samples were obtained at 0, 1, 2 and 4 hours post-exposure via tail-vein puncture $(N=6)$. Statistics were analyzed with two-way ANOVA $(P \leq 0.05),{ }^{*}$ Sham control group vs. nano- $\mathrm{TiO}_{2}$ exposed groups.

Figure 3. Nano- $\mathrm{TiO}_{2}$ exposure also increases T-Helper type II cytokines 4 hours postexposure in bronchoalveolar lavage fluid (BALF). Multiplex cytokine analysis showing concentrations of IL-4, IL-5, IL-13 in BALF from exposed and control animals 4 hours postexposure $(\mathrm{N}=6)$. Statistics were analyzed with two-way ANOVA ( $\mathrm{P} \leq 0.05)$, * Sham control group vs. nano- $\mathrm{TiO}_{2}$ exposed groups.

Figure 4. Nano- $\mathrm{TiO}_{2}$ exposure is associated with an increase in pulmonary interleukin-33 levels. Lung sections from control $(\mathrm{A})$ and nano- $\mathrm{TiO}_{2}$ exposed $(\mathrm{B})$ animals obtained 4 hours postexposure were stained for interleukin-33. Fluorescence was achieved by staining tracheal sections with an anti-IL-33-FITC conjugated antibody. Relative fluorescence is shown in (C)

tagged antibody ( $\mathrm{N}=6-7)$. Statistics were analyzed with two-way ANOVA $(\mathrm{P} \leq 0.05),{ }^{*}$ Sham control group vs. nano- $\mathrm{TiO}_{2}$ exposed groups.

Figure 5. Pre-treatment of nano- $\mathrm{TiO}_{2}$ - exposed animals with an anti-IL-33 antibody lowers BALF and plasma IL-33. Interleukin-33 levels in (A) BALF and (B) plasma in control, nano- $\mathrm{TiO}_{2}$ 
exposed groups and in rats pre-treated with an anti-IL-33 antibody. Rats were pre-treated with an anti-IL-33 antibody (1 mg/kg) 30 minutes prior to exposure. Plasma and BALF IL-33 levels were measured 4 hours post-exposure. Statistics were analyzed with two-way ANOVA (P $\leq 0.05)$. * Sham control group vs. nano- $\mathrm{TiO}_{2}$ exposed groups, $\mp \mathrm{P}<0.05$ Exposed + Anti-IL-33 Antibody vs Exposed.

Figure 6. Flow cytometric analysis of lung-resident Group II innate lymphoid cells. Flow cytometry of lung tissue 4 hours post-exposure for Group II Innate Lymphoid Cells in (A) control and $(\mathrm{B})$ nano- $\mathrm{TiO}_{2}$ exposed animals $(\mathrm{N}=6)$.

Figure 7. Nano- $\mathrm{TiO}_{2}$ exposure increases pulmonary ILC2 but not ILC1. Quantification of percentage of total pulmonary cells of (A) ILC1 and (B) ILC2 in control and nano- $\mathrm{TiO}_{2}$ exposed animals $(\mathrm{N}=6)$. Statistics were analyzed with two-way ANOVA $(\mathrm{P} \leq 0.05),{ }^{*}$ Sham control group vs. nano- $\mathrm{TiO}_{2}$ exposed groups.

Figure 8. $\mathrm{Nano}^{-\mathrm{TiO}_{2}}$ exposure increases NF-kB phosphorylation at Serine-468 in isolated ILC2. Quantification of NF-kB phosphorylation at (A) serine-468 and (B) serine 536 in isolated ILC2 from control and nano- $\mathrm{TiO}_{2}$ exposed animals $(\mathrm{N}=6)$. Statistics were analyzed with two-way ANOVA ( $\mathrm{P} \leq 0.05),{ }^{*}$ Sham control group vs. nano- $\mathrm{TiO}_{2}$ exposed groups.

Figure 9. Th2 cytokine secretion in isolated ILC2 is increased by nano- $\mathrm{TiO}_{2}$ exposure. Multiplex cytokine analysis showing concentrations of IL-4, IL-5, IL-13 supernatant from isolated ILC2 cultured overnight from control and nano- $\mathrm{TiO}_{2}$ exposed animals $(\mathrm{N}=6)$. Statistics were analyzed with two-way ANOVA ( $\mathrm{P} \leq 0.05)$, * Sham control group vs. nano- $\mathrm{TiO}_{2}$ exposed groups. 
Figure 10. Endothelium-dependent dilation of radial arterioles is blunted by nano- $\mathrm{TiO}_{2}$ exposure and improved by pre-treatment with an anti-IL-33 antibody. Endotheliumdependent dilation of uterine radial arterioles from control, exposed and anti-IL-33 antibody treated animals was determined using pressure myography $(n=12-18)$. All vascular assessments were performed 24 hours post-exposure. Statistics were analyzed with two-way ANOVA (P $\leq$ 0.05). * Sham control group vs. nano- $\mathrm{TiO}_{2}$ exposed groups, $\mp \mathrm{P}<0.05$ Exposed + Anti-IL-33 Antibody vs Exposed. 


\section{Reference List:}

[1] Abukabda, A.B., et al., Heterogeneous Vascular Bed Responses to Pulmonary Titanium Dioxide Nanoparticle Exposure. Front Cardiovasc Med, 2017. 4: p. 33.

[2] Artis, D. and H. Spits, The biology of innate lymphoid cells. Nature, 2015. 517(7534): p. 293301.

[3] Baisch, B.L., et al., Equivalent titanium dioxide nanoparticle deposition by intratracheal instillation and whole body inhalation: the effect of dose rate on acute respiratory tract inflammation. Part Fibre Toxicol, 2014. 11: p. 5.

[4] Beamer, C.A., et al., IL-33 mediates multi-walled carbon nanotube (MWCNT)-induced airway hyper-reactivity via the mobilization of innate helper cells in the lung. Nanotoxicology, 2013. 7(6): p. 1070-81.

[5] Bonner, J.C., M. Hoffman, and A.R. Brody, Alpha-macroglobulin secreted by alveolar macrophages serves as a binding protein for a macrophage-derived homologue of plateletderived growth factor. Am J Respir Cell Mol Biol, 1989. 1(3): p. 171-9.

[6] Deckers, J., F. Branco Madeira, and H. Hammad, Innate immune cells in asthma. Trends Immunol, 2013. 34(11): p. 540-7.

[7] Drake, L.Y. and H. Kita, Group 2 innate lymphoid cells in the lung. Adv Immunol, 2014. 124: p. 1-16.

[8] Duerr, C.U. and J.H. Fritz, Isolation of Group 2 Innate Lymphoid Cells from Mouse Lungs. Methods Mol Biol, 2017. 1656: p. 253-261.

[9] Engler-Chiurazzi, E.B., et al., Impacts of prenatal nanomaterial exposure on male adult Sprague-Dawley rat behavior and cognition. J Toxicol Environ Health A, 2016. 79(11): p. 44752.

[10] Halim, T.Y. and F. Takei, Isolation and characterization of mouse innate lymphoid cells. Curr Protoc Immunol, 2014. 106: p. 325 1-13. 
[11] Halim, T.Y.F. and A.N.J. McKenzie, New kids on the block: group 2 innate lymphoid cells and type 2 inflammation in the lung. Chest, 2013. 144(5): p. 1681-1686.

[12] Hams, E. and P.G. Fallon, Innate type 2 cells and asthma. Curr Opin Pharmacol, 2012. 12(4): p. 503-9.

[13] Hams, E., et al., IL-25 and type 2 innate lymphoid cells induce pulmonary fibrosis. Proc Natl Acad Sci U S A, 2014. 111(1): p. 367-72.

[14] Hong, J.T., NF-kB as a mediator of brain inflammation in AD. CNS Neurol Disord Drug Targets, 2017.

[15] Jackson, P., et al., Prenatal exposure to carbon black (printex 90): effects on sexual development and neurofunction. Basic Clin Pharmacol Toxicol, 2011. 109(6): p. 434-7.

[16] Johansson, H.K.L., et al., Airway exposure to multi-walled carbon nanotubes disrupts the female reproductive cycle without affecting pregnancy outcomes in mice. Part Fibre Toxicol, 2017. 14(1): p. 17.

[17] Kabata, H., et al., Group 2 innate lymphoid cells and asthma. Allergol Int, 2015. 64(3): p. 227-34.

[18] Katwa, P., et al., A carbon nanotube toxicity paradigm driven by mast cells and the IL(3)(3)/ST(2) axis. Small, 2012. 8(18): p. 2904-12.

[19] Lai, D.M., Q. Shu, and J. Fan, The origin and role of innate lymphoid cells in the lung. Mil Med Res, 2016. 3: p. 25.

[20] LeBlanc, A.J., et al., Nanoparticle inhalation impairs endothelium-dependent vasodilation in subepicardial arterioles. J Toxicol Environ Health A, 2009. 72(24): p. 1576-84.

[21] Ley, K., The second touch hypothesis: T cell activation, homing and polarization. F1000Res, 2014. 3: p. 37.

[22] Liu, X., et al., Anti-IL-33 antibody treatment inhibits airway inflammation in a murine model of allergic asthma. Biochem Biophys Res Commun, 2009. 386(1): p. 181-5 
[23] Look, M., et al., The nanomaterial-dependent modulation of dendritic cells and its potential influence on therapeutic immunosuppression in lupus. Biomaterials, 2014. 35(3): p. 1089-95.

[24] Marsland, B.J., et al., Innate signals compensate for the absence of PKC-\{theta\} during in vivo CD8(+) T cell effector and memory responses. Proc Natl Acad Sci U S A, 2005. 102(40): p. $14374-9$.

[25] McKenzie, A.N., Type-2 innate lymphoid cells in asthma and allergy. Ann Am Thorac Soc, 2014. 11 Suppl 5: p. S263-70.

[26] Michaudel, C., et al., Inflammasome, IL-1 and inflammation in ozone-induced lung injury. Am J Clin Exp Immunol, 2016. 5(1): p. 33-40.

[27] Mjosberg, J.M., et al., Human IL-25- and IL-33-responsive type 2 innate lymphoid cells are defined by expression of CRTH2 and CD161. Nat Immunol, 2011. 12(11): p. 1055-62.

[28] Monticelli, L.A., et al., Innate lymphoid cells promote lung-tissue homeostasis after infection with influenza virus. Nat Immunol, 2011. 12(11): p. 1045-54.

[29] Nichols, C.E., et al., Reactive oxygen species damage drives cardiac and mitochondrial dysfunction following acute nano-titanium dioxide inhalation exposure. Nanotoxicology, 2018. 12(1): p. $32-48$.

[30] Nurkiewicz, T.R., et al., Nanoparticle inhalation augments particle-dependent systemic microvascular dysfunction. Part Fibre Toxicol, 2008. 5: p. 1.

[31] Nurkiewicz, T.R., et al., Pulmonary nanoparticle exposure disrupts systemic microvascular nitric oxide signaling. Toxicol Sci, 2009. 110(1): p. 191-203.

[32] Pichery, M., et al., Endogenous IL-33 is highly expressed in mouse epithelial barrier tissues, lymphoid organs, brain, embryos, and inflamed tissues: in situ analysis using a novel II-33-LacZ gene trap reporter strain. J Immunol, 2012. 188(7): p. 3488-95.

[33] Pradere, J.P., et al., Negative regulation of NF-kappaB p65 activity by serine 536 phosphorylation. Sci Signal, 2016. 9(442): p. ra85. 
[34] Pugazhenthi, S., et al., Induction of an inflammatory loop by interleukin-1beta and tumor necrosis factor-alpha involves NF-kB and STAT-1 in differentiated human neuroprogenitor cells. PLoS One, 2013. 8(7): p. e69585.

[35] Sanfins, E., et al., Nanoparticle effect on neutrophil produced myeloperoxidase. PLoS One, 2018. 13(1): p. e0191445.

[36] Schmitz, J., et al., IL-33, an interleukin-1-like cytokine that signals via the IL-1 receptorrelated protein ST2 and induces T helper type 2-associated cytokines. Immunity, 2005. 23(5): p. $479-90$.

[37] Shaw, J.L., et al., IL-33-responsive innate lymphoid cells are an important source of IL-13 in chronic rhinosinusitis with nasal polyps. Am J Respir Crit Care Med, 2013. 188(4): p. $432-$ 9.

[38] Sonnenberg, G.F., et al., SnapShot: innate lymphoid cells. Immunity, 2013. 39(3): p. 622622 e1.

[39] Spits, H. and J.P. Di Santo, The expanding family of innate lymphoid cells: regulators and effectors of immunity and tissue remodeling. Nat Immunol, 2011. 12(1): p. 21-7.

[40] Spits, H., et al., Innate lymphoid cells--a proposal for uniform nomenclature. Nat Rev Immunol, 2013. 13(2): p. 145-9.

[41] Stapleton, P.A., et al., Estrous cycle-dependent modulation of in vivo microvascular dysfunction after nanomaterial inhalation. Reprod Toxicol, 2018. 78: p. 20-28.

[42] Stapleton, P.A., et al., Impairment of coronary arteriolar endothelium-dependent dilation after multi-walled carbon nanotube inhalation: a time-course study. Int J Mol Sci, 2012. 13(11): p. $13781-803$.

[43] Stapleton, P.A., et al., Maternal engineered nanomaterial exposure and fetal microvascular function: does the Barker hypothesis apply? Am J Obstet Gynecol, 2013. 209(3): p. 227 e1-11. 
[44] Stapleton, P.A., et al., Maternal engineered nanomaterial inhalation during gestation alters the fetal transcriptome. Part Fibre Toxicol, 2018. 15(1): p. 3.

[45] Stapleton, P.A., et al., Uterine microvascular sensitivity to nanomaterial inhalation: An in vivo assessment. Toxicol Appl Pharmacol, 2015. 288(3): p. 420-8.

[46] Stier, M.T., et al., IL-33 promotes the egress of group 2 innate lymphoid cells from the bone marrow. J Exp Med, 2018. 215(1): p. 263-281.

[47] Stone, K.C., et al., Distribution of lung cell numbers and volumes between alveolar and nonalveolar tissue. Am Rev Respir Dis, 1992. 146(2): p. 454-6.

[48] Takatori, H., et al., Lymphoid tissue inducer-like cells are an innate source of IL-17 and IL-22. J Exp Med, 2009. 206(1): p. 35-41.

[49] Taylor, A.J., et al., Atomic layer deposition coating of carbon nanotubes with aluminum oxide alters pro-fibrogenic cytokine expression by human mononuclear phagocytes in vitro and reduces lung fibrosis in mice in vivo. PLoS One, 2014. 9(9): p. e106870.

[50] Thompson, E.A., et al., Innate Immune Responses to Nanoparticle Exposure in the Lung. J Environ Immunol Toxicol, 2014. 1(3): p. 150-156.

[51] Turner, J.E., et al., IL-9-mediated survival of type 2 innate lymphoid cells promotes damage control in helminth-induced lung inflammation. J Exp Med, 2013. 210(13): p. 295165.

[52] Vercelli, D., J. Gozdz, and E. von Mutius, Innate lymphoid cells in asthma: when innate immunity comes in a Th2 flavor. Curr Opin Allergy Clin Immunol, 2014. 14(1): p. 29-34.

[53] Wang, X., et al., Intravenously delivered graphene nanosheets and multiwalled carbon nanotubes induce site-specific Th2 inflammatory responses via the IL-33/ST2 axis. Int J Nanomedicine, 2013. 8: p. 1733-48.

[54] Wang, X., et al., Multi-walled carbon nanotube instillation impairs pulmonary function in C57BL/6 mice. Part Fibre Toxicol, 2011. 8: p. 24. 
[55] Xu, J., et al., IL33-mediated ILC2 activation and neutrophil IL5 production in the lung response after severe trauma: A reverse translation study from a human cohort to a mouse trauma model. PLoS Med, 2017. 14(7): p. e1002365.

[56] Yang, Q., et al., Group 2 innate lymphoid cells mediate ozone-induced airway inflammation and hyperresponsiveness in mice. J Allergy Clin Immunol, 2016. 137(2): p. 5718.

[57] Yasuda, K., et al., Contribution of IL-33-activated type II innate lymphoid cells to pulmonary eosinophilia in intestinal nematode-infected mice. Proc Natl Acad Sci U S A, 2012. 109(9): p. 3451-6.

[58] Yoda, K., et al., Stochastic Secretion Response to Transient Stimulus of Goup-2 Innate Lymphoid Cells (ILC2). IEEE, 2017.

[59] Zeng, S., et al., IL-33 Receptor (ST2) Signalling is Important for Regulation of Th2Mediated Airway Inflammation in a Murine Model of Acute Respiratory Syncytial Virus Infection. Scand J Immunol, 2015. 81(6): p. 494-501.

[60] Zoltowska, A.M., et al., The interleukin-33 receptor ST2 is important for the development of peripheral airway hyperresponsiveness and inflammation in a house dust mite mouse model of asthma. Clin Exp Allergy, 2016. 46(3): p. 479-90. 


\section{Table 1: Animal Characteristics}

\begin{tabular}{|c|c|c|c|c|c|c|c|}
\hline & $\mathrm{N}$ & $\begin{array}{c}\text { Age } \\
(\text { weeks) }\end{array}$ & $\begin{array}{c}\text { Weight } \\
(\mathrm{grams})\end{array}$ & $\begin{array}{c}\text { Heart } \\
\text { Rate } \\
(\mathrm{bpm})\end{array}$ & $\begin{array}{c}\text { MAP } \\
(\mathrm{mm} \mathrm{Hg})\end{array}$ & $\begin{array}{c}\text { Systolic } \\
\text { Blood } \\
\text { Pressure } \\
(\mathrm{mm} \mathrm{Hg})\end{array}$ & $\begin{array}{c}\text { Diastolic Blood } \\
\text { Pressure (mm Hg) }\end{array}$ \\
\hline $\begin{array}{c}\text { Sham- } \\
\text { Control }\end{array}$ & 13 & $14 \pm 1$ & $232 \pm 5$ & $319 \pm 13$ & $85 \pm 3$ & $103 \pm 4$ & $73 \pm 2$ \\
\hline $\begin{array}{c}\text { Nano- } \\
\text { TiO } \\
\text { Exposed }\end{array}$ & 14 & $15 \pm 1$ & $249 \pm 12$ & $330 \pm 9$ & $86 \pm 4$ & $110 \pm 6$ & $78 \pm 4$ \\
\hline
\end{tabular}




\section{Table 2: Arteriolar Characteristics}

\begin{tabular}{|c|c|c|c|c|c|c|c|}
\hline & $\mathrm{n}$ & $\begin{array}{c}\text { Inner } \\
\text { diameter } \\
(\mu \mathrm{m})\end{array}$ & $\begin{array}{c}\text { Outer } \\
\text { diameter } \\
(\mu \mathrm{m})\end{array}$ & $\begin{array}{c}\text { Tone } \\
(\%)\end{array}$ & $\begin{array}{c}\text { Passive } \\
\text { diameter inner } \\
(\mu \mathrm{m})\end{array}$ & $\begin{array}{c}\text { Passive diameter } \\
\text { outer }(\mu \mathrm{m})\end{array}$ & $\begin{array}{c}\text { Wall Tension } \\
(\text { Newton/meter })\end{array}$ \\
\hline Control & 18 & $98 \pm 5$ & $152 \pm 14$ & $26 \pm 7$ & $152 \pm 9$ & $199 \pm 15$ & $0.33 \pm 0.1$ \\
\hline Exposed & 16 & $102 \pm 11$ & $164 \pm 13$ & $21 \pm 4$ & $159 \pm 15$ & $207 \pm 17$ & $0.34 \pm 0.1$ \\
\hline $\begin{array}{c}\text { Exposed }+ \\
\text { anti-IL-33 }\end{array}$ & 12 & $97 \pm 11$ & $158 \pm 19$ & $21 \pm 4$ & $140 \pm 14$ & $187 \pm 26$ & $0.31 \pm 0.6$ \\
\hline
\end{tabular}


Figure 1:

A

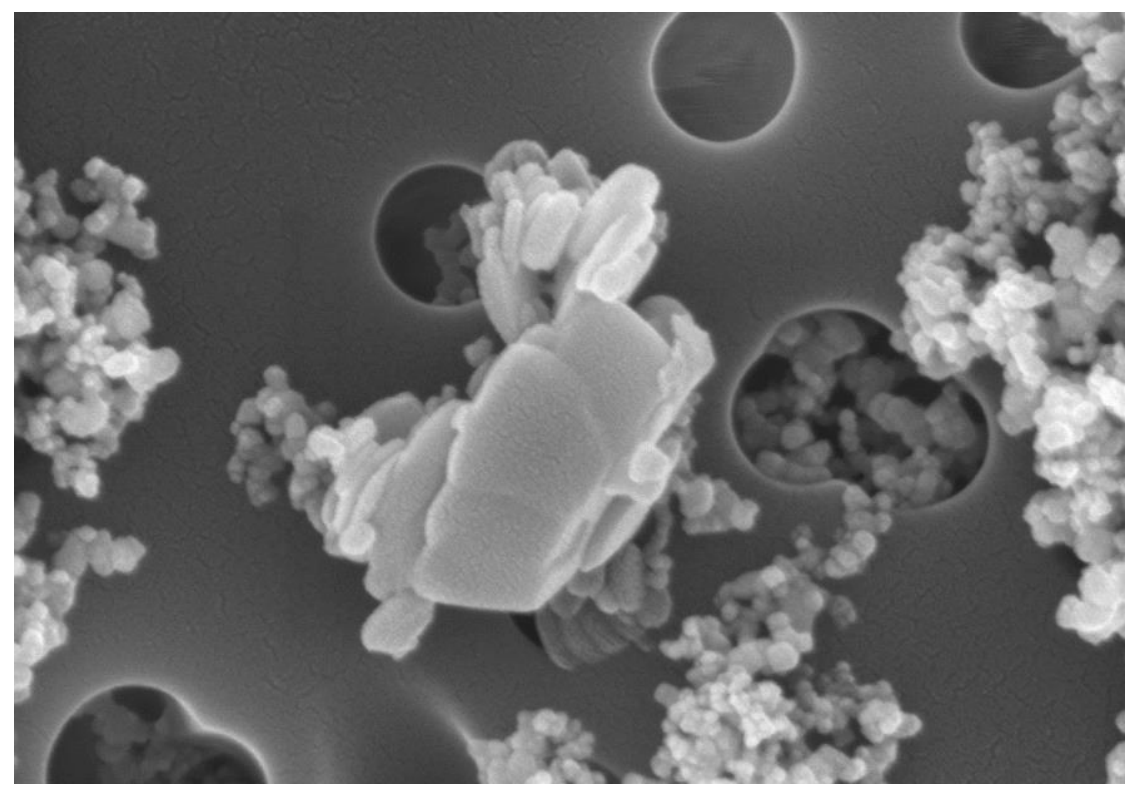

B

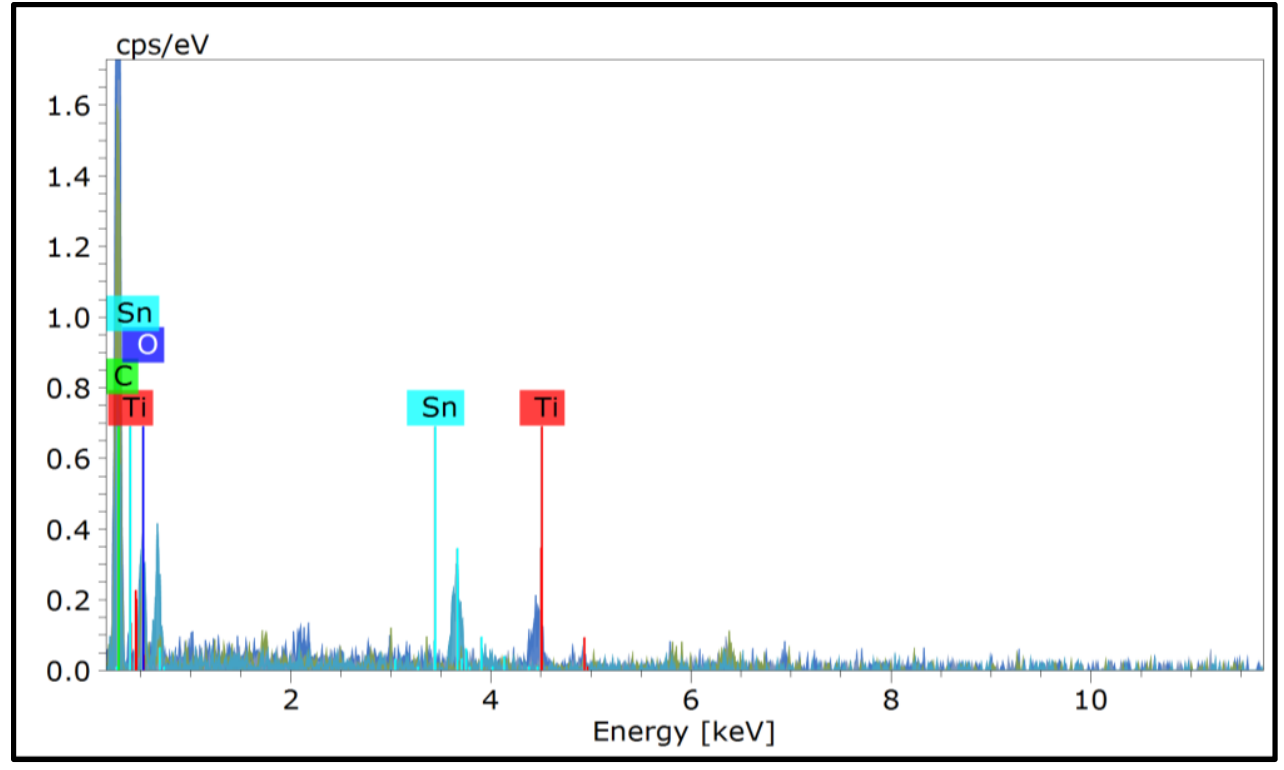


Figure 2:

A

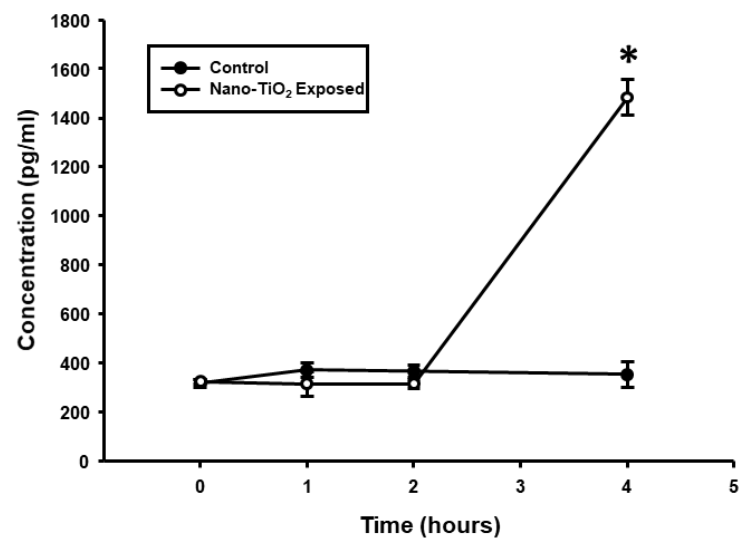

C

TNF- $\alpha$

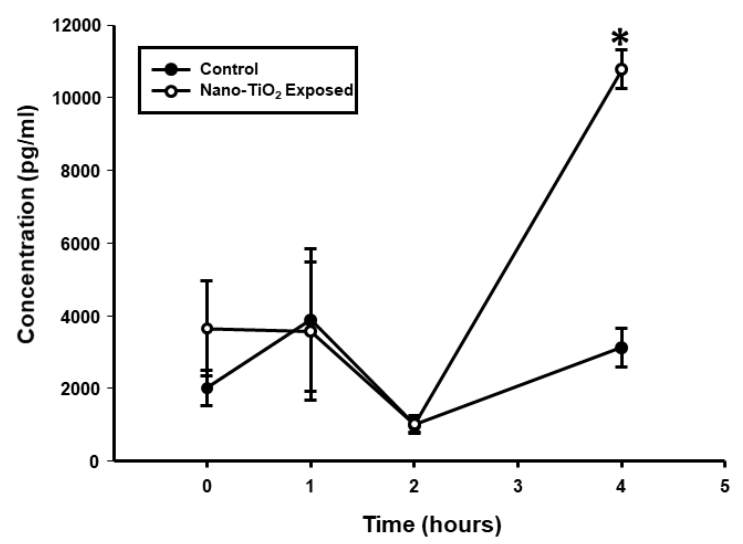

B

IL-1 $\beta$

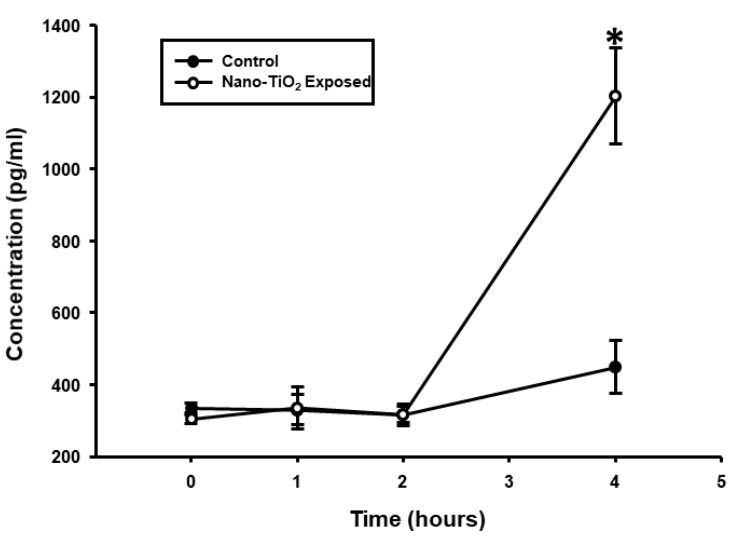

D

IL-13

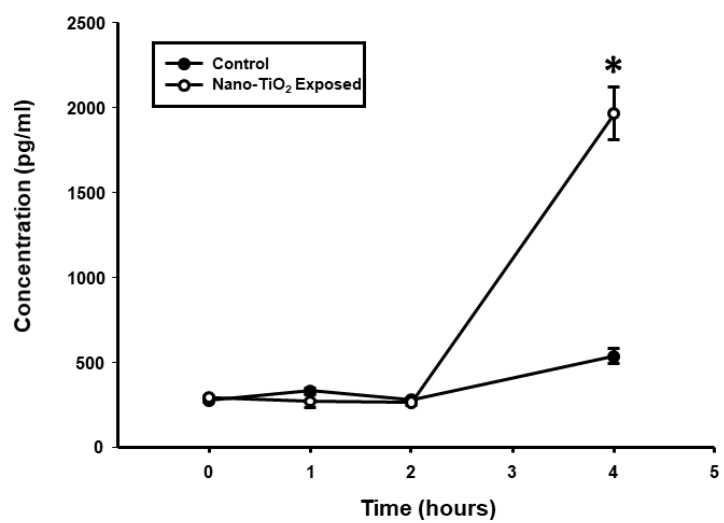

E

IL-5

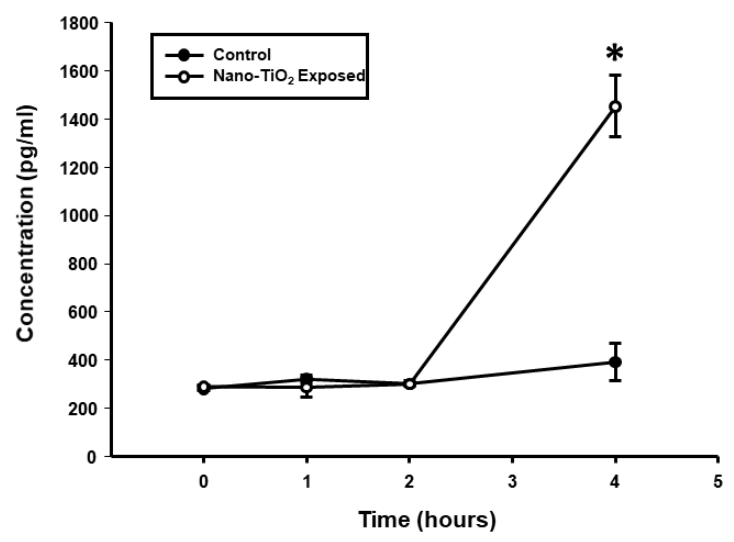


Figure 3:

\section{Broncholalveolar Lavage}

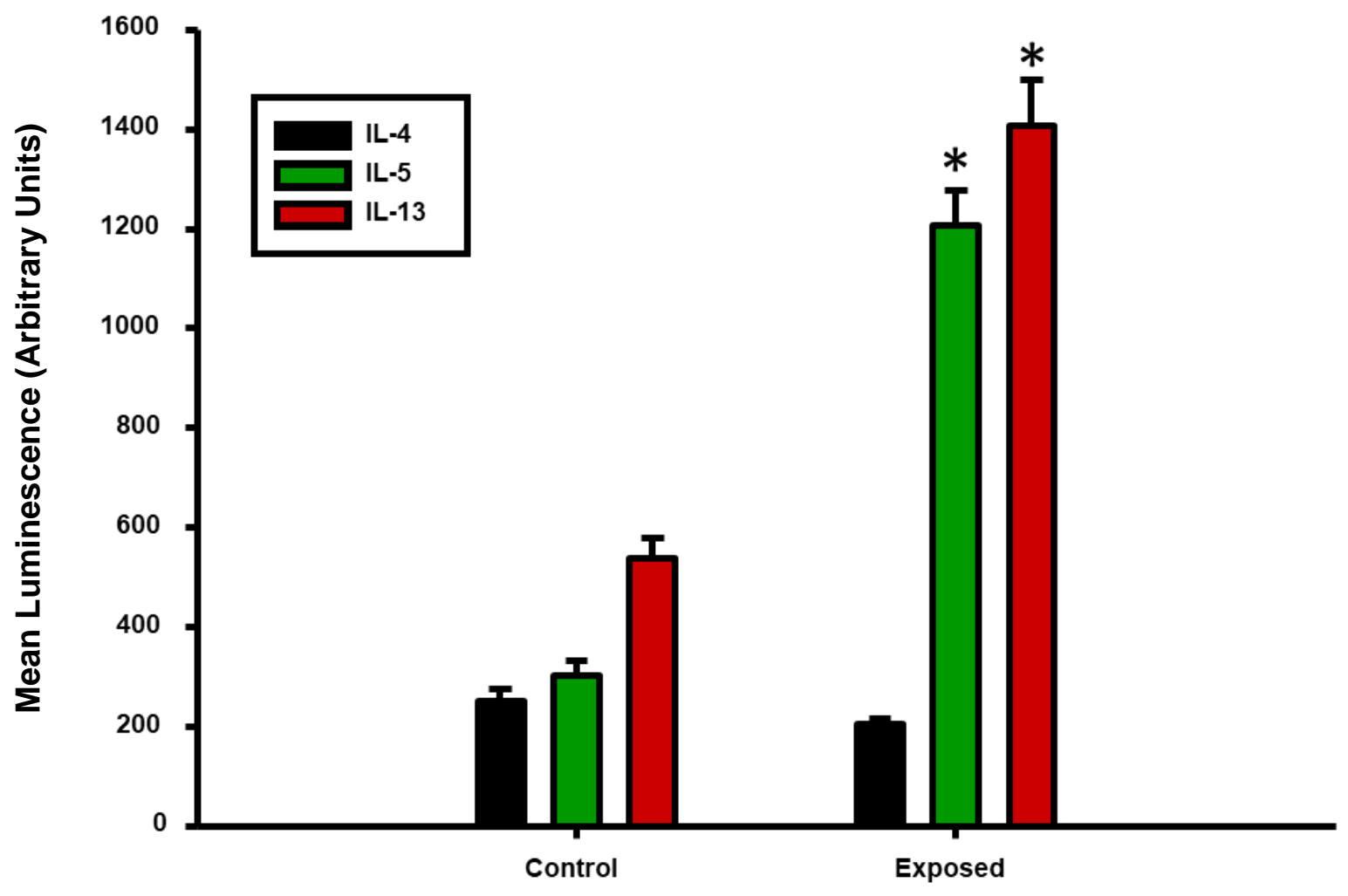


Figure 4:
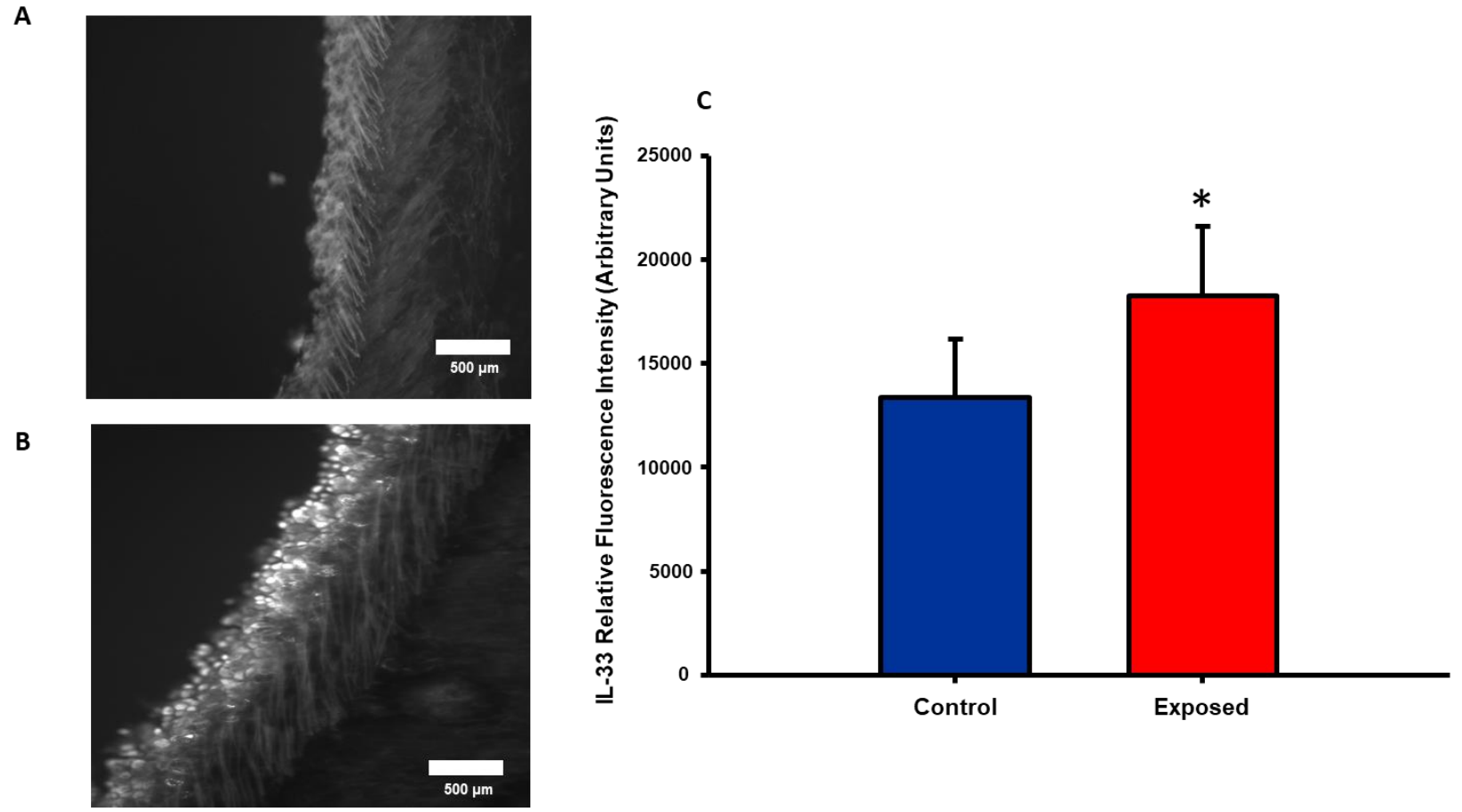
Figure 5:

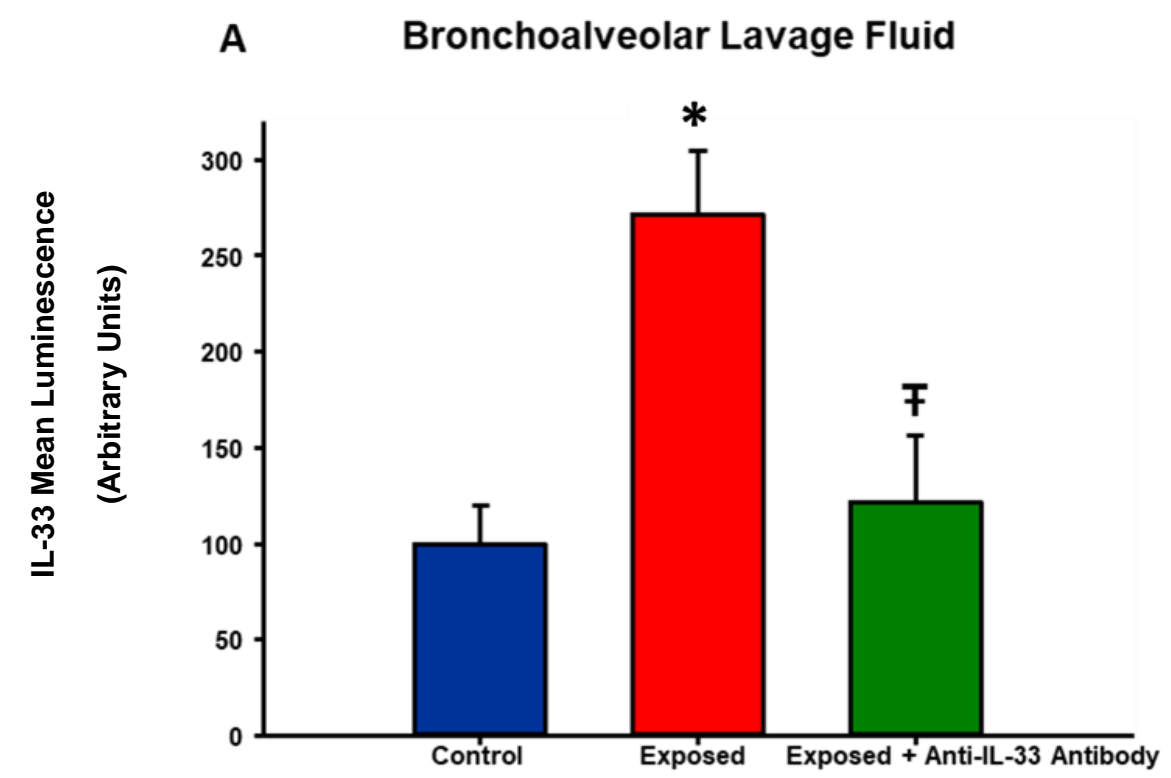

B

Plasma

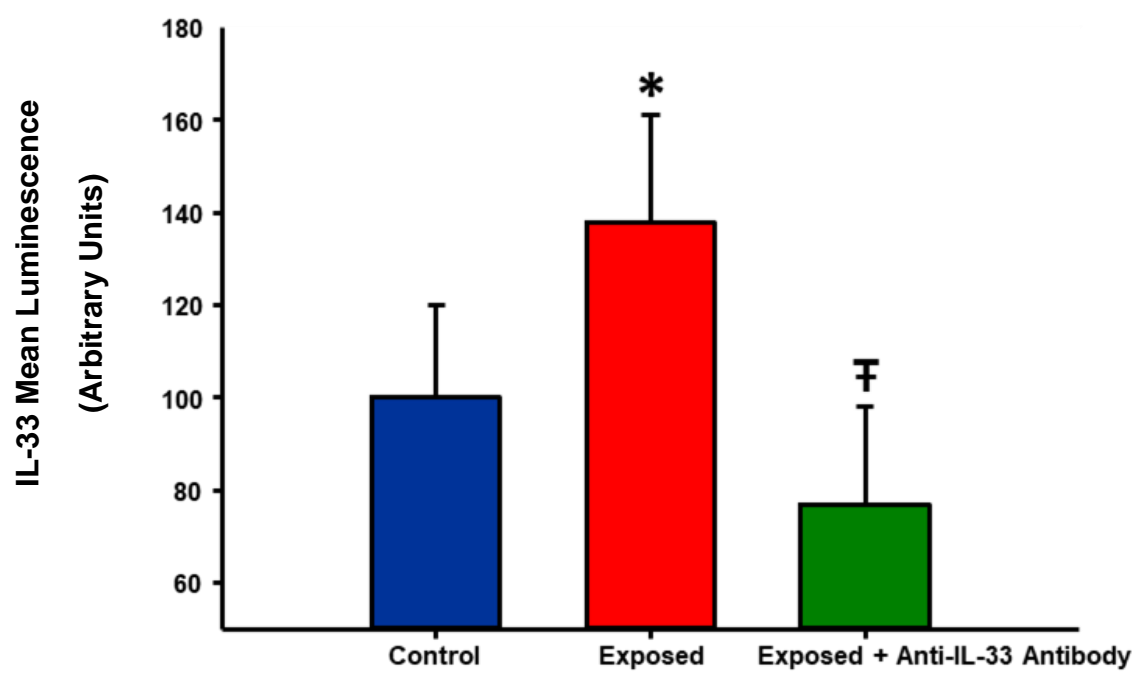


Figure 6:

\section{A) Control - ILC2}

1

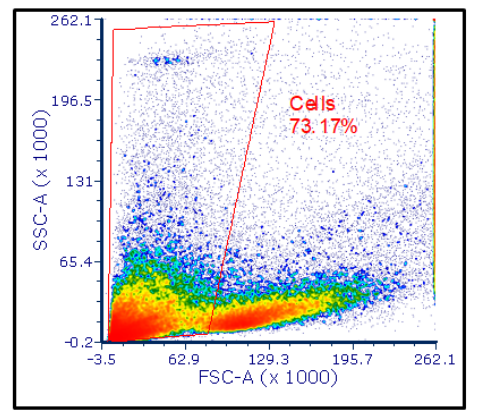

2

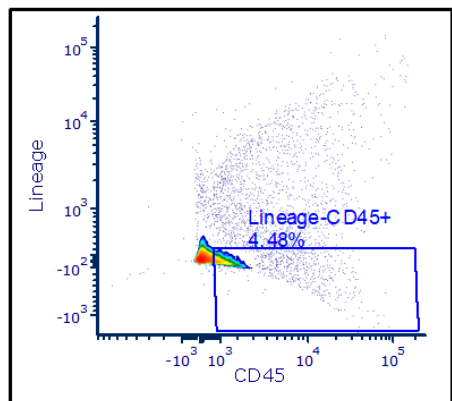

4

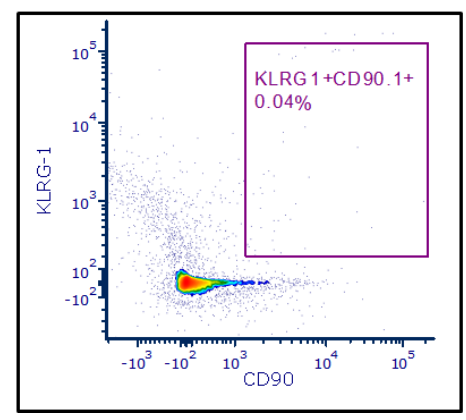

B) Exposed - ILC2

1

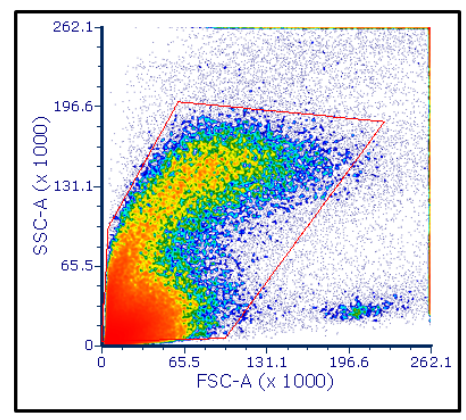

2

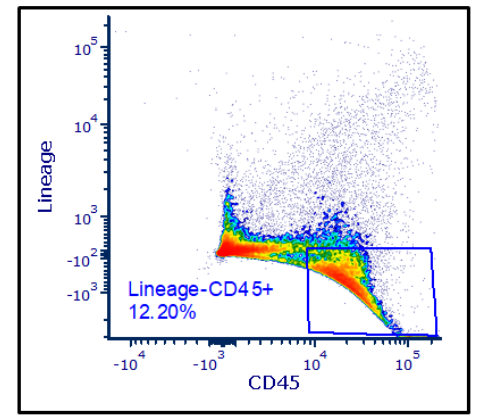

4

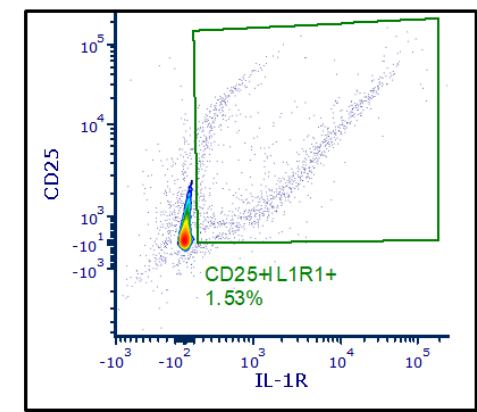

3

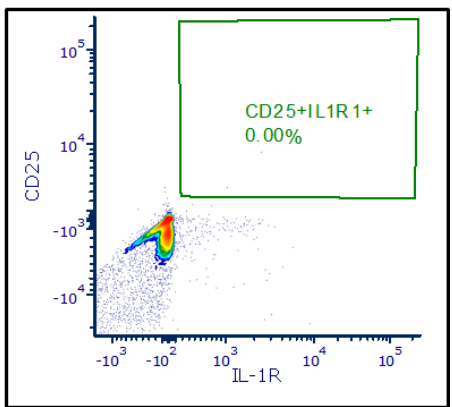

3

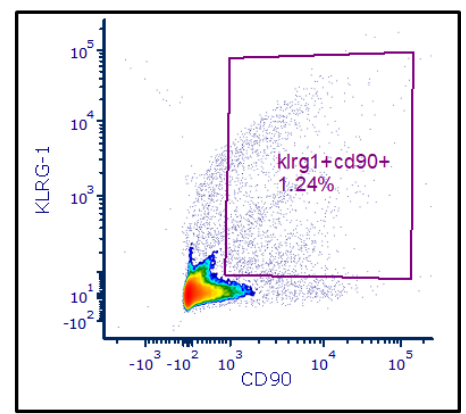


Figure 7:

A Group 1 Innate Lymphoid Cells

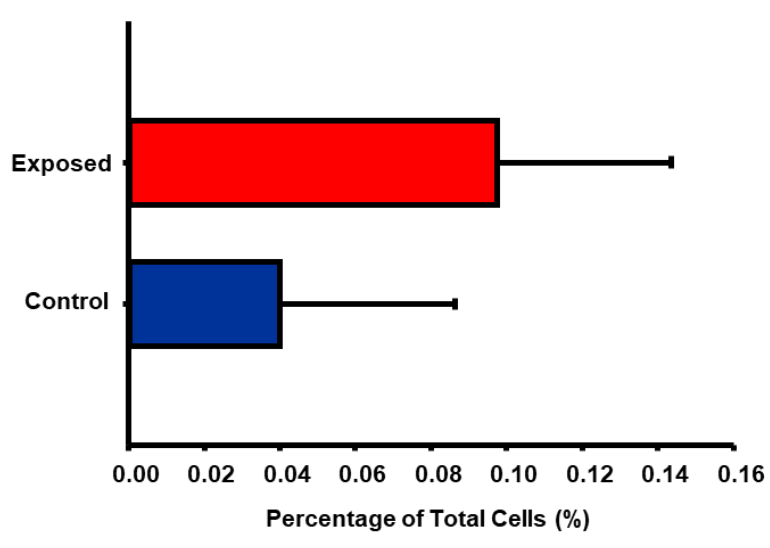

B Group 2 Innate Lymphoid Cells

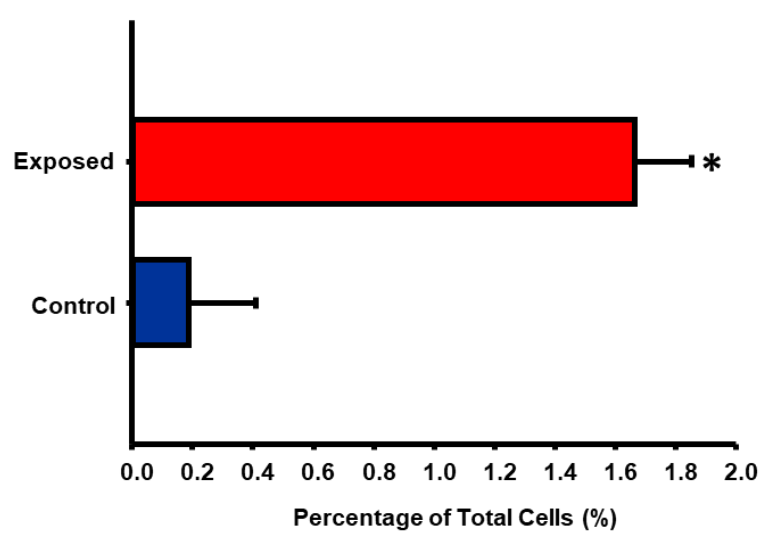


Figure 8:
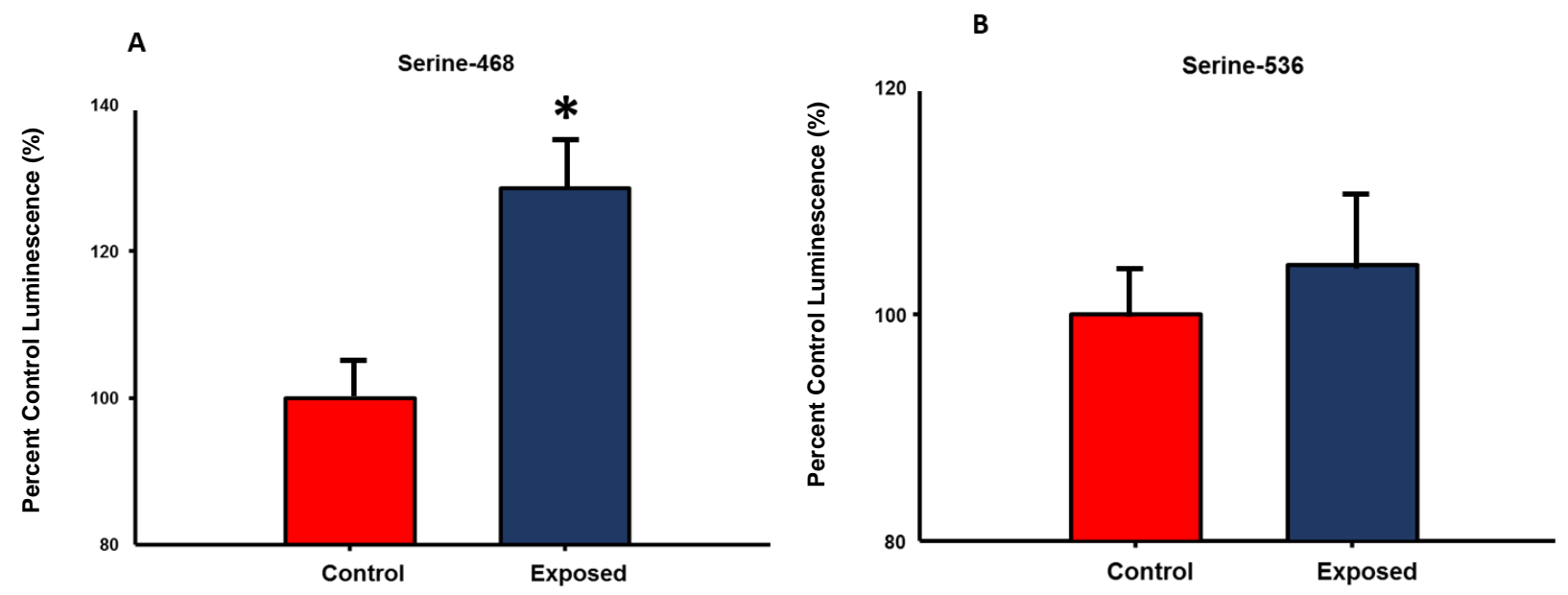
Figure 9:

\section{Cell Supernatant}

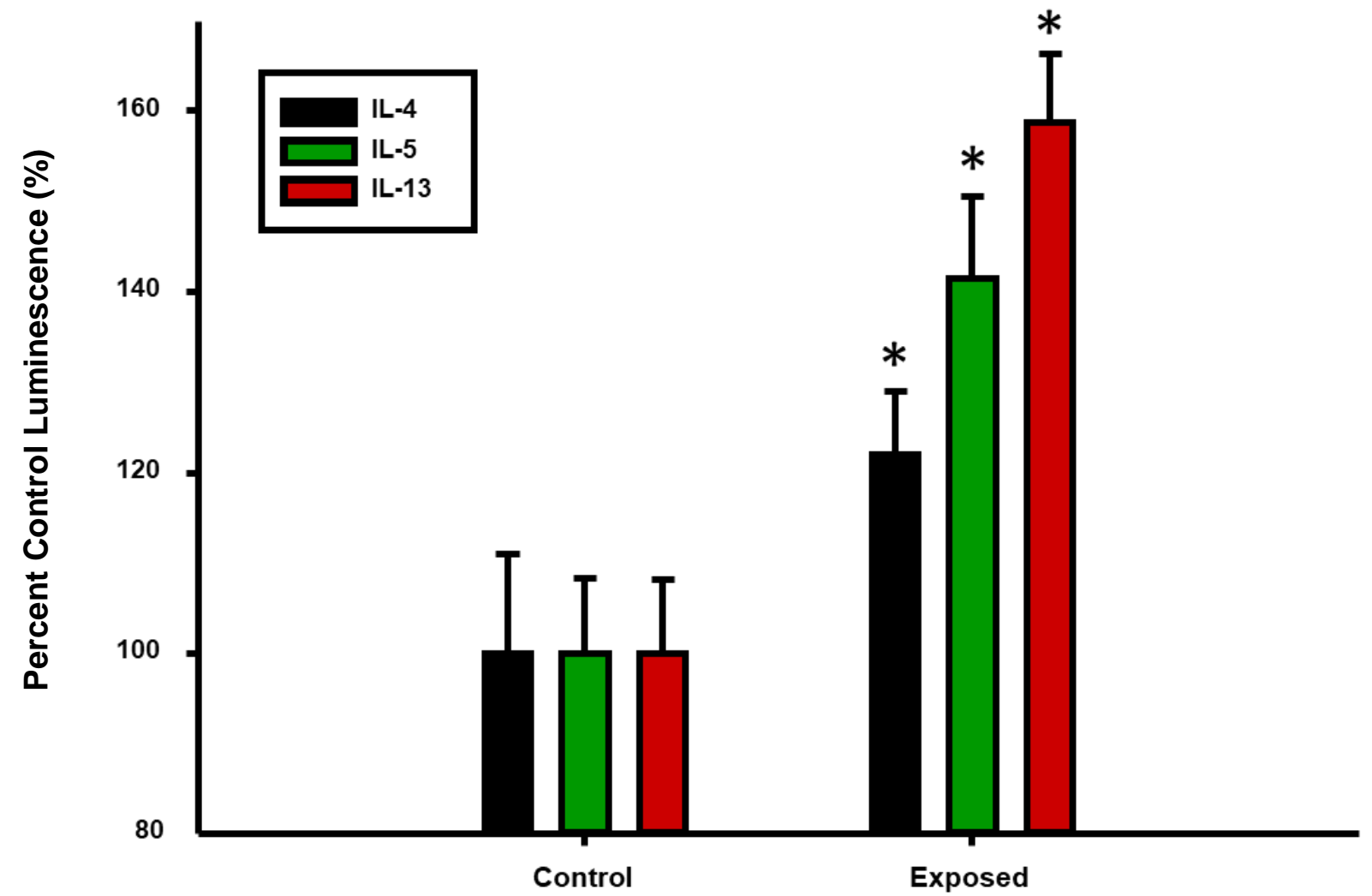


Figure 10:

\section{Endothelium - Dependent Dilation - Radial Arterioles}

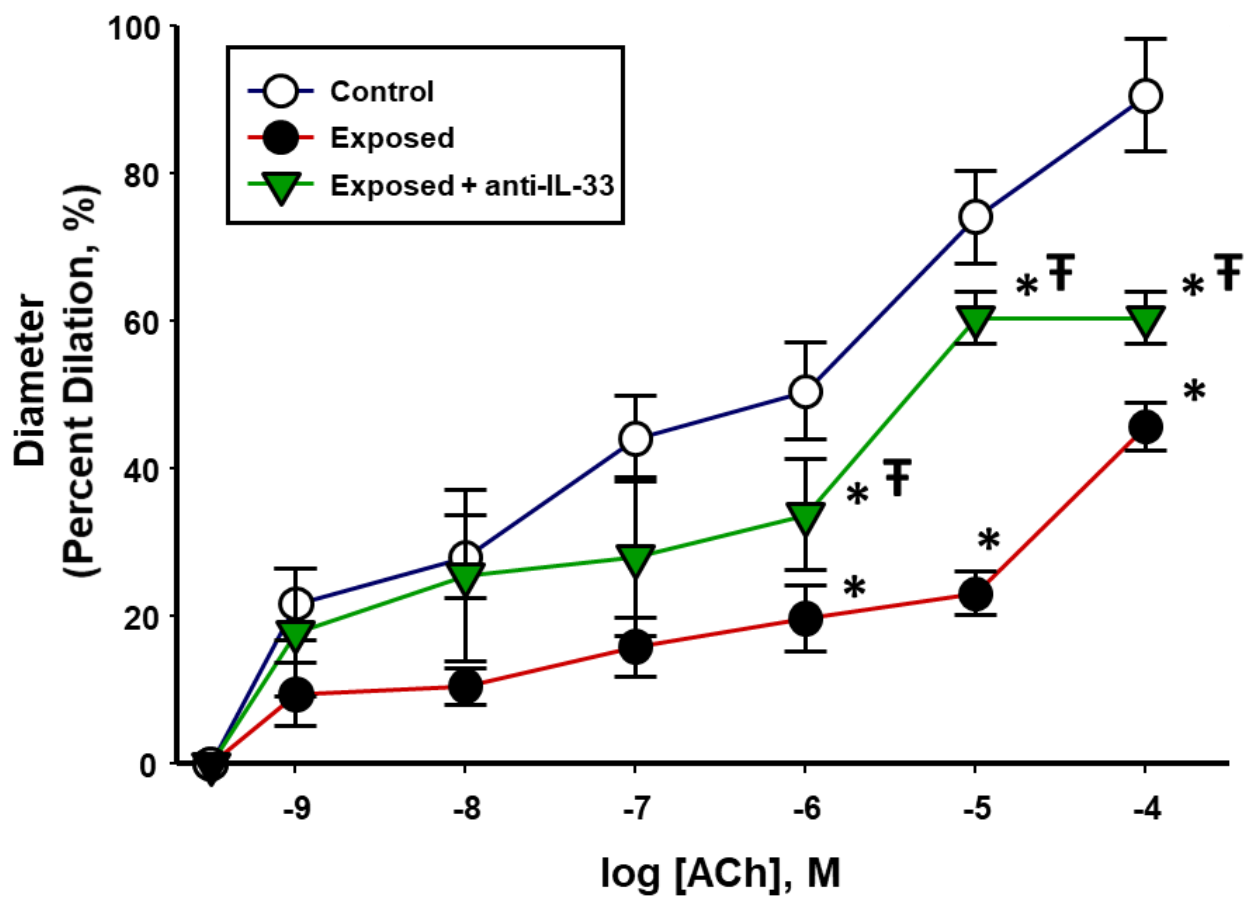




\section{Chapter 4}

Maternal Engineered Nanomaterial Inhalation Exposure Compromises Placental Hemodynamics

Running Title: Nanoparticles and the Placenta

\section{Authors:}

Abukabda, A.B. ${ }^{1,2}$

Bowdridge, E.C. ${ }^{1,2}$

McBride, C.R. ${ }^{1,2}$

Batchelor, T.P. ${ }^{1,2}$

Goldsmith, W.T.1,2

Garner, K.L. ${ }^{1,2}$

Friend, $\mathrm{S}^{3}$

Nurkiewicz, T.R. ${ }^{1,2}$

\section{Affiliations:}

${ }^{1}$ Department of Physiology and Pharmacology, West Virginia University School of Medicine, Morgantown, WV, USA.

${ }^{2}$ Toxicology Working Group, West Virginia University School of Medicine, Morgantown, WV, USA.

${ }^{3}$ National Institute for Occupational Safety and Health, Morgantown, WV, USA. 


\section{Abstract:}

The fetal consequences of gestational engineered nanomaterial (ENM) exposure are unclear. The placenta is a barrier protecting the fetus and allowing transfer of substances from the maternal circulation. The purpose of this study was to determine the effects of maternal pulmonary titanium dioxide nanoparticle (nano-TiO2) exposure on the placenta and umbilical vascular reactivity. We hypothesized that pulmonary nano-TiO2 inhalation exposure increases placental vascular resistance and impairs umbilical vascular responsiveness. Pregnant Sprague-Dawley rats were exposed via whole-body inhalation to nano-TiO2 with an aerodynamic diameter of 188 $\pm 0.4 \mathrm{~nm}$. On gestational day (GD) 11 , rats began inhalation exposures (6h/exposure). Daily lung deposition was $88 \pm 3 \mu \mathrm{g}$. Animals were exposed for 6 days for a cumulative lung burden of $525 \pm 16 \mu \mathrm{g}$. On GD 20 placentas, umbilical artery and vein were isolated, cannulated, and treated with acetylcholine (ACh), angiotensin II (ANGII), S-Nitroso-N-acetyl-DL-penicillamine (SNAP), or calcium-free superfusate (Ca2+-free). Mean outflow pressure was measured in isolated placental units. ACh increased mean outflow pressures to $53 \pm 5 \mathrm{~mm} \mathrm{Hg}$ in sham-controls but only to $35 \pm 4$ $\mathrm{mm} \mathrm{Hg}$ in exposed subjects. ANGIl decreased outflow pressure in placentas from exposed animals $(17 \pm 7 \mathrm{~mm} \mathrm{Hg})$ compared to sham-controls $(31 \pm 6 \mathrm{~mm} \mathrm{Hg})$. Ca2+-free superfusate yielded maximal outflow pressures in sham-control $(63 \pm 5 \mathrm{~mm} \mathrm{Hg})$ and exposed $(30 \pm 10 \mathrm{~mm} \mathrm{Hg})$ rats. Umbilical artery endothelium-dependent dilation was decreased in nano-TiO2 exposed fetuses $(30 \pm 9 \%)$ compared to sham-controls (58 $\pm 6 \%$ ), but ANGII sensitivity was increased ($79 \pm 20 \%$ vs $-36 \pm 10 \%$ ). These results indicate that maternal gestational pulmonary nano-TiO2 exposure increases placental vascular resistance and impairs umbilical vascular reactivity.

Keywords: engineered nanomaterials, titanium dioxide nanoparticles, microcirculation, placenta 


\section{Introduction:}

While the field of nanotechnology has seen a tremendous expansion and growth in recent years [20], a more complete understanding of maternal and fetal effects associated with ENM exposure warrants further investigation and is of key relevance for the safe design and regulation of industrially relevant nanomaterials. Pregnancy represents a sensitive window and is characterized by profound adaptive physiological changes [18], particularly of the maternal-fetal circulation. These changes include vasculogenesis, the de novo formation of blood vessels by endothelial progenitor cells and angiogenesis, the development of new vessels from pre-existing ones. All these mechanisms cause an increase in overall vascular diameter and number which results in a significant reduction in the vascular resistance of the maternal-fetal circulation. Expansive remodeling of the maternal circulation is a crucial and fundamental process for a successful pregnancy and any impairment in this finely regulated series of events may have catastrophic effects on maternal and fetal health. Indeed, insufficient growth and development of the uteroplacental circulation results in placental under-perfusion and has been associated with intrauterine growth restriction (IUGR) [13] and pre-eclampsia [7].

The study of developmental and reproductive effects of ENM in various animal models has in recent years gained more attention $[4,12]$. We have reported that in rats, inhalation of $11.3 \mathrm{mg} / \mathrm{m}^{3}$ of nano- $\mathrm{TiO}_{2}$ during late gestation resulted in an impairment in endothelium-dependent reactivity of fetal vessels, while offspring survival was severely comprised by inhalation of a higher dose for 11 days [25]. Similarly, inhalation of $10.6 \mathrm{mg} / \mathrm{m}^{3}$ nano- $\mathrm{TiO}_{2}$ during late gestation (average 6.8 days, $5 \mathrm{~h}$ /day) was associated with an impairment of endothelium-dependent dilation and active mechano-transduction in uterine arteries and a reduction in maximal mitochondrial respiration in female offspring [24]. Additionally, reduced pup weight and litter size [2] along with persistent 
cognitive deficits of gestationally exposed young adult rats have also been reported [5], thus highlighting the potential transgenerational impact of gestational ENM exposure.

One of the critical components of the maternal-fetal vascular axis is the placenta. The placenta is a transient barrier organ with significant functions that are crucial for fetal development. The placental cellular barrier at the maternal-fetal interface consists of trophoblasts and endothelial cells [11]. Between these two cell layers, the surrounding tissue is formed primarily by stromal fibroblasts and macrophages (Hofbauer cells). The importance of the placenta is underscored by its role in the fetal regulation of gas and heat transfer, excretion, metabolism, synthesis and secretion of endocrine hormones, hematopoiesis and immunity [11]. Additionally, the placenta maintains the balance between the hydrostatic and oncotic pressures in the capillary microcirculation and the interstitial fluid. At full term, the placenta receives $>90 \%$ of uterine blood flow as a result of significant hemodynamic adaptations occurring primarily due to a significant decrease in vascular resistance caused by extensive remodeling of the maternal uterine microvasculature, a decrease in vascular tone and an increase in the vasodilatory sensitivity of the maternal-fetal circulations [18].

Since the development of nanotechnology, the question of maternal-fetal particle translocation has been present. In vitro, fluorescently labelled carboxylate-modified polystyrene beads with a diameter of up to $500 \mathrm{~nm}$ have been shown to cross the placental barrier and induce trophoblast apoptosis [10]. Silica nanoparticles having a diameter of less than $100 \mathrm{~nm}$ and nano- $\mathrm{TiO}_{2}$ with a diameter of approximately $35 \mathrm{~nm}$ have been shown to cross the placental barriers, potentially resulting in impaired placental function by decreasing the area of the trophoblasts and the length of the villi in the labyrinthine layer of the placenta [9]. 
The contribution of placental and maternal-fetal vascular dysfunction in the developmental origin of disease and health cannot be over-emphasized. Yet, there is a paucity of in-vivo data regarding the effects of pulmonary ENM exposure on placental vascular resistance because it is essentially impossible to directly assess these variables. Therefore, the purpose of this study was to determine ex-vivo the effects of nano- $\mathrm{TiO}_{2}$ exposure on placental hemodynamics and on the function of the umbilical vasculature. We hypothesized that maternal pulmonary nano- $\mathrm{TiO}_{2}$ exposure results in a significant increase in placental vascular resistance and an impairment in umbilical vascular reactivity. We adopted and modified an established ex-vivo perfusion placental model [6] to make direct assessments of placental outflow pressure and placental flow rate measured as a function of the inflow pressure. Vascular reactivity of the umbilical artery and vein were directly assessed via pressure myography. 


\section{Methods:}

\section{Nanomaterial Characterization}

Nano- $\mathrm{TiO}_{2}$ P25 powder, obtained from Evonik (Aeroxide $\mathrm{TiO}_{2}$, Parsippany, $\mathrm{NJ}$ ), has previously been shown to be a mixture composed primarily of anatase $(80 \%)$ and rutile $(20 \%) \mathrm{TiO}_{2}$, with a primary particle size of $21 \mathrm{~nm}$ and a surface area of $48.08 \mathrm{~m}^{2} / \mathrm{g}$, and a Zeta-potential of $-56.6 \mathrm{mV}$ [26]. Analysis of the elemental composition of the nano- $\mathrm{TiO}_{2}$ was conducted via energy dispersive spectroscopy.

Scanning and transmission electron microscopy (SEM) procedures were performed using a nano$\mathrm{TiO}_{2}$ exposed 0.2 um polycarbonate filter with a 200 mesh formvar coated copper grid affixed in the center. A wedge-shaped portion of the polycarbonate filter was mounted onto a $13 \mathrm{~mm}$ aluminum stub using carbon double stick tape. The sample was sputter coated with goldpalladium for two minutes. The sample was imaged at $5 \mathrm{KeV}$ using a Hitachi $\mathrm{S} 4800$ field-emission scanning electron microscope (Tokyo, Japan). For transmission electron microscopy, the exposed grid was removed from the polycarbonate filter, and imaged at $80 \mathrm{KeV}$ on a JEOL 1400 transmission electron microscope (Tokyo, Japan).

\section{Experimental Animals and Whole-Body Inhalation Exposure}

Female Sprague - Dawley rats (8-10 weeks) were purchased from Hilltop Laboratories (Scottdale, PA) and housed in an AAALAC approved facility at West Virginia University (WVU) with 12:12 h light - dark cycle and regulated temperature. Rats were allowed ad libitum access to food and water. All procedures were approved by the Institutional Animal Care and Use Committee of WVU. 
Nano- $\mathrm{TiO}_{2}$ aerosols were generated using a customized high-pressure acoustical generator (HPAG) and then allowed to enter the whole-body exposure chamber. A personal DataRAM (pDR-1500; Thermo Environmental Instruments Inc., Franklin, MA) was utilized to sample the exposure chamber air to determine the average mass concentration in real-time. Feedback loops within the software automatically adjusted the acoustic energy to maintain a stable mass concentration during the exposure. A target DataRAM concentration of $10 \mathrm{mg} / \mathrm{m}^{3}$ for a period of 6 hours was selected as previously described [26]. A concurrent gravimetric measurement indicated an average mass concentration of approximately $10 \mathrm{mg} / \mathrm{m}^{3}$ during the 6 hour exposure period. Aerosol size distributions were examined from the exposure chamber with: 1) a highresolution electrical low-pressure impactor (ELPI+; Dekati, Tampere, Finland), (2) a scanning particle mobility sizer (SMPS 3938; TSI Inc., St. Paul, MN), and (3) an aerodynamic particle sizer (APS 3321; TSI Inc., St. Paul, MN). Bedding material soaked with water was used in the exposure chamber to maintain a comfortable humidity during the exposure. Sham-control animals were exposed to HEPA filtered air only with similar chamber conditions in terms of temperature and humidity.

To allow for normal implantation and neovascularization, inhalation exposures were initiated on GD 11 and lasted for 6 days. Exposure days were not consecutive to decrease animal stress. Once the steady state nano- $\mathrm{TiO}_{2}$ aerosol concentration was achieved, the exposure duration was adjusted to produce a daily calculated lung deposition of $31 \pm 1.1 \mu \mathrm{g}$ per day, and the cumulative, calculated dose was therefore $217 \pm 1.0 \mu \mathrm{g}$. Lung deposition was calculated based on previously described mouse methodology, and normalized to rat weight and to pregnant rat minute ventilation using the equation: $D=F \cdot V \cdot C \cdot T$, where $F$ is the deposition fraction (14\%), $V$ is the minute ventilation based on body weight, $C$ equals the mass concentration $\left(\mathrm{mg} / \mathrm{m}^{3}\right)$, and T equals the exposure duration (minutes) [31]. The target concentration was $10 \mathrm{mg} / \mathrm{m}^{3}$ and the duration 
was 4-6 h/exposure (depending on the steady state concentration, as this was used to calculate the lung burden). The last exposure was conducted $24 \mathrm{~h}$ prior to sacrifice and experimentation. Control animals were exposed to HEPA filtered air only.

\section{Collection and Ex Vivo Perfusion of the Rat Placenta}

Rats were anesthetized with isoflurane gas (5\% induction, $2-3.5 \%$ maintenance). The animals were placed on a heating pad to maintain a $37^{\circ} \mathrm{C}$ rectal temperature. The trachea was intubated to ensure an open airway and the right carotid artery was cannulated to allow blood pressure measurements and sampling. Following blood sampling, the abdominal cavity of the animal was opened and the uterine horns were externalized. A placenta with a large and intact portion of the uterine artery was selected, excised and placed in a dissecting dish containing PSS at $4^{\circ} \mathrm{C}$. The amniotic sac was subsequently opened and the placenta was separated from the fetus. The vitelline artery was removed from the umbilical cord which was severed as close as possible to the fetus, preserving the umbilical vasculature for cannulation. The maternal circulation was then tied off using 9-0 silk sutures (Living Systems Instrumentation, Burlington, VT). After separation of the umbilical artery and vein, the placenta was then transferred to an isolated microvessel chamber (Living Systems Instrumentation, Burlington, VT).

Both the umbilical artery and vein were cannulated between two glass pipettes (Outer diameter: 175-200 $\mu \mathrm{m}$, Living Systems Instrumentation, Burlington, VT), and tied with 9-0 silk sutures in the chamber. The placenta was perfused with PSS and the chamber was superfused with fresh oxygenated $\left(21 \% \mathrm{O}_{2} / 5 \% \mathrm{CO}_{2}\right)$ PSS and warmed to $37^{\circ} \mathrm{C}$. After a 30 -minute equilibration period, the inflow pressure was increased in a stepwise fashion $(0 \mathrm{~mm} \mathrm{Hg}, 20 \mathrm{~mm} \mathrm{Hg}, 40 \mathrm{~mm} \mathrm{Hg}, 60$ $\mathrm{mm} \mathrm{Hg}$ and $80 \mathrm{~mm} \mathrm{Hg}$ ). Outflow pressure and flow rate were measured in placentas in normal 
superfusate and following pre-treatment with acetylcholine (ACh:10-2), S-Nitroso-N-acetyl-DLpenicillamine (SNAP: 10-2), angiotensin II (ANGII: $10^{-2}$ ) and calcium-free superfusate.

\section{Immunohistochemical Staining of Placental Tissue}

Uteri from Sham controls and nano- $\mathrm{TiO}_{2}$ exposed animals were removed immediately after sacrifice and two placentas per rat $(n=3)$ were dissected away from the pup and the uterine wall. The placental tissue was immediately placed into a fixative (4\% paraformaldehyde) for 3 hours at $4^{\circ} \mathrm{C}$ and then transferred to PBS overnight. Twelve hours later, the tissue was flash frozen and stored at $-80^{\circ} \mathrm{C}$ until sectioned. Placentas were sectioned at $50 \mu \mathrm{m}$ through the center of the placenta. On day 1 of the protocol, the sections were washed $4 \times 5$ minutes in $0.1 \mathrm{M}$ PBS to remove excess cryoprotectant and washed overnight at $4^{\circ} \mathrm{C}$. The next day, the sections were washed $4 \times 5$ minutes in PBS, placed in $1 \% \mathrm{H}_{2} \mathrm{O}_{2}$ for 10 minutes and subsequently washed $4 \times$ 5 minutes in PBS. The tissue was then incubated for at least 1 hour in a blocking solution containing PBS, 20\% Triton X-100 (PBSTX) (Sigma-Aldrich, St Louis, MO, USA) and 20\% normal goat serum (NGS) (Jackson ImmunoResearch Laboratories, Inc., West Grove, PA, USA) in PBS. Tissue sections were placed in a solution containing rabbit anti-Von Willebrand factor (vWFcatalogue number ab6994; dilution 1:2 000; AbCam, Cambridge, MA) in PBSTX and 4\% NGS for 16 hours. After incubation with the primary antibody, sections were incubated in a solution containing biotinylated goat anti-rabbit $\lg G$ and a solution containing avidin-biotin horseradishperoxidase conjugate (Vectastain Elite ABC; dilution 1:600; Vector Laboratories) for 1 hour. Sections were then washed and incubated for 10 minutes in biotinylated tyramine (TSA) (dilution 1:250; Perkin Elmer, Waltham, MA, USA) in PBS containing $3 \% \mathrm{H}_{2} \mathrm{O}_{2}$ per $1 \mathrm{~mL}$ of solution. After washing, sections were incubated in a solution containing DyLight green 488-streptavidin (1:200, Fisher Scientific) for 1 hour followed by washes and incubation in PBSTX and 4\% NGS for at least 1 hour. Sections were incubated in mouse anti-smooth muscle actin (SMA-catalogue number ab 
7817; dilution 1:1000; AbCam, Cambridge, MA) in PBSTX and 4\% NGS for 16 hours. The next day, sections were incubated in Alexa555 goat anti-rabbit (dilution 1:200; Life Technologies, Carlsbad, CA, USA) for 1 hour, washed, mounted on Superfrost slides (Fisher Scientific), covered with a coverslip using ProLong Diamond Antifade Mountant with DAPI (Thermo Fisher, Waltham, MA) and stored in the dark at $4^{\circ} \mathrm{C}$ until analysis.

\section{Pressure Myography Vessel Preparation}

A placental unit was placed in a dissecting dish with PSS maintained at $4^{\circ} \mathrm{C}$. The umbilical cord was cut longitudinally to reveal the artery and vein. The umbilical artery and vein were isolated, transferred to a vessel chamber, cannulated between two glass pipettes, and tied with silk sutures in the chamber (Living Systems Instrumentation, Burlington, VT). The chamber was superfused with fresh oxygenated $\left(21 \% \mathrm{O}_{2} / 5 \% \mathrm{CO}_{2}\right)$ PSS and warmed to $37^{\circ} \mathrm{C}$. Both vessels were pressurized to $60 \mathrm{~mm} \mathrm{Hg}$ using a servo control system and extended to their in-situ length. Internal and external vascular diameters were measured using video calipers (Colorado Video, Boulder, $\mathrm{CO})$.

\section{Vascular Reactivity}

Vessels were allowed to develop spontaneous tone, defined as the degree of constriction experienced by a blood vessel relative to its maximally dilated state. Vascular tone ranges from $0 \%$ (maximally dilated) to $100 \%$ (maximal constriction). After equilibration, various parameters of vascular function were analyzed.

Endothelium-Dependent Dilation — vessels were exposed to increasing concentrations of ACh $\left(10^{-9}-10^{-4} \mathrm{M}\right)$ added to the vessel chamber. 
Endothelium-Independent Dilation-increasing concentrations of SNAP $\left(10^{-9}-10^{-4} \mathrm{M}\right)$ were used to assess vascular smooth muscle responsiveness.

Vasoconstriction - vessels were exposed to increasing concentrations of ANGII $\left(10^{-9}-10^{-4} \mathrm{M}\right)$. The steady state diameter of the vessel was recorded for at least 2 min after each dose. After each dose curve was completed, the vessel chamber was washed to remove excess chemicals by carefully removing the superfusate and replacing it with fresh warmed oxygenated PSS. After all experimental treatments were complete, the PSS was replaced with $\mathrm{Ca}^{2+}$-free PSS until maximum passive diameter was established.

\section{Pressure Myography Calculations}

Data are expressed as means \pm standard error. Spontaneous tone was calculated by the following equation:

$$
\text { Spontaneous tone }(\%)=\left\{\frac{(D m-D i)}{D i}\right\} \times 100
$$

, where $\mathrm{Dm}$ is the maximal diameter and $\mathrm{Di}$ is the initial steady state diameter recorded prior to the experiment. Active responses to pressure were normalized to the maximal diameter using the following formula:

$$
\text { Normalized diameter }=D s s / D m
$$

, where Dss is the steady state diameter recorded during each pressure change. The experimental responses to $\mathrm{ACh}, \mathrm{PE}$, and SNP are expressed using the following equation:

$$
\text { Diameter }(\text { percent maximal diameter })=\left\{\frac{(D s s-D c o n)}{(D m-D c o n)}\right\} \times 100
$$


, where DCon is the control diameter recorded prior to the dose curve, DSS is the steady state diameter at each dose of the curve. The experimental response to PE is expressed using the following equation:

$$
\text { Diameter (percent maximal diameter })=\left\{\frac{(\text { Dcon }- \text { Dss })}{(\text { Dcon })}\right\} \times 100
$$

Wall thickness (WT) was calculated from the measurement of both inner (ID) and outer (OD) steady state diameters at the end of the $\mathrm{Ca}^{2+}$ free wash using the following equation:

$$
W T=(O D-I D) / 2
$$

Wall-to-lumen ratio (WLR) was calculated using the following equation:

$$
W L R=W T / I D
$$

\section{Statistics}

Point-to-point differences in the dose response curves of the umbilical vessels were evaluated using two-way repeated measures analysis of variance (ANOVA) with a Tukey`s post-hoc analysis when significance was found. The animal characteristics and vessel characteristics were analyzed using a one-way ANOVA with a Tukey post-hoc analysis when significance was found. A mixed-effects analysis was used to determine the effect of exposure on placental outflow pressure (GraphPad Prism; San Diego, CA). Wherever sphericity (equal variance) was not met, the Greenhouse-Geiger correction was adopted. A mix-model linear regression was also performed to determine the differences in the slopes between the sham-control and nano-TiO2 exposed groups. Significance was set at $p<0.05, \mathrm{n}$ is the number of vessels and placentas, while $\mathrm{N}$ is the number of animals. 


\section{Results}

\section{SEM Images and Mass Spectrometry of $\mathrm{Nano}^{-\mathrm{TiO}_{2}}$}

Figure 1 A shows field-emission scanning electron microscope images (Hitachi S4800, Tokyo, Japan) of the nano- $\mathrm{TiO}_{2}$ suspension used for this study. The SMPS and APS data were combined to determine the Geometric Count Median Diameter using the log normal distribution obtained with the log probability plot method and was determined to be $190 \mathrm{~nm}$ with a standard error of 0.5 (Figure 1 B). The ELPI High Resolution data indicated a Geometric Count Median Diameter of $188 \mathrm{~nm}$ with a standard error of the mean of 0.4 (Figure $1 \mathrm{C}$ ). The aerosol concentration of nano$\mathrm{TiO}_{2}$ was determined to be $10 \pm 0.1 \mathrm{mg} / \mathrm{m}^{3}$ (Figure $1 \mathrm{D}$ ).

\section{Animal and Vessel Characteristics}

No significant changes were observed in the age, body weight or number of pups between control and exposure groups (Table 1). Additionally, umbilical artery and vein inner and outer diameter, tone, wall thickness, wall to lumen ratio and calculated wall tension were not affected by nano$\mathrm{TiO}_{2}$ inhalation (Tables 2 and 3). Interestingly, the umbilical vein passive inner diameter was significantly increased in the maternal nano- $\mathrm{TiO}_{2}$ exposed group $(623 \pm 40 \mu \mathrm{m})$ compared to the sham-control group $(543 \pm 29 \mu \mathrm{m})$. From these results we can infer that maternal nano- $\mathrm{TiO}_{2}$ inhalation may potentially alter vascular wall anatomy in the umbilical macrocirculation.

\section{Ex Vivo Perfusion of the Rat Placenta}

Figure 2 A displays a representative ex vivo perfused placenta preparation. An intact placenta (3) was cannulated between 2 glass pipets via the umbilical artery (1) and the umbilical vein (2). The input (arterial) perfusion pressure was held constant $(0-80 \mathrm{~mm} \mathrm{Hg})$ while vasoactive stimuli 
were added. Output pressure and flow rate $(\mu \mathrm{L} / \mathrm{min})$ were the dependent variable representing placental resistance. Vascular diameter was also measured with video calipers. Figure 2 B shows a representative image of the traces obtained using the ex vivo perfused placenta preparation. Panel 1 shows the inflow pressure via the umbilical artery (independent variable), while panels 2 and 3 show the outflow pressure and the perfusate placental flow rate (dependent variables). Outflow pressure is the result of placental vascular resistance, while placental perfusate flow is a function of the pressure differential between the umbilical artery and the umbilical vein. No differences were noted in the placental perfusion flow rate at baseline and following treatment with the different vasoactive agents (data not shown).

The outflow pressures in the sham-control and maternal nano- $\mathrm{TiO}_{2}$ exposed placentas were compared. In the normal superfusate, the sham-control placentas displayed a significantly higher outflow pressure compared to those from exposed dams when the inflow pressure was increased in a stepwise fashion (Figure $3 \mathbf{A}$ ). These results confirm that gestational nano- $\mathrm{TiO}_{2}$ exposure increases placental vascular resistance. Similarly, sham-control placentas displayed higher mean outflow pressures after treatment with $\mathrm{ACh}$ compared to nano- $\mathrm{TiO}_{2}$ exposed placentas (Figure 3 B), indicating that the observed increase in placental vascular resistance may be due to an impairment of endothelium-dependent dilation. Figure 4 A and 4 B show that pulmonary gestational nano- $\mathrm{TiO}_{2}$ exposure did not affect placental endothelial-independent dilation, while ANGII sensitivity was significantly augmented. Placentas were then treated with calcium-free superfusate (Figure 5) to measure maximum vascular dilation. In this case, placentas from nanoTiO2 exposed dams presented a significantly decreased output pressure compared to the shamcontrol group. Lastly, Figure 6 shows the mean outflow pressure at $80 \mathrm{~mm} \mathrm{Hg}$ before and following treatment with different vasoactive agents. The mean outflow pressure in pre-treated sham-control placentas was $43 \pm 1 \mathrm{~mm} \mathrm{Hg}$, while those from exposed animals were $32 \pm 3 \mathrm{~mm}$ 
Hg. ACh-treated placentas presented mean outflow pressures of $53 \pm 5 \mathrm{~mm} \mathrm{Hg}$ in sham-controls and $35 \pm 4 \mathrm{~mm} \mathrm{Hg}$ in exposed subjects. SNAP treatment led to mean outflow pressures of $42 \pm 8$ in sham-controls and $32 \pm 9$ in nano-TiO2 $\mathrm{mm} \mathrm{Hg}$. Treatment with ANGIl resulted in a significantly decreased outflow pressure in the placentas from exposed animals $(17 \pm 7 \mathrm{~mm} \mathrm{Hg})$ compared to sham-control placentas $(31 \pm 6 \mathrm{~mm} \mathrm{Hg})$. Lastly, calcium-free superfusate yielded outflow pressures in sham-control and exposed rats of $63 \pm 5 \mathrm{~mm} \mathrm{Hg}$ and $29 \pm 10 \mathrm{~mm} \mathrm{Hg}$ respectively. Linear regression was used to further confirm that these slopes and therefore the physiologic continuum of the pressure-flow responses were significantly different between the sham-control and exposed groups (Supplementary Figures 1-3).

\section{Endothelium-Dependent Dilation}

Endothelium-dependent dilation of the umbilical artery was significantly impaired following nano$\mathrm{TiO}_{2}$ exposure (Figure $7 \mathrm{~A}$ ), with a mean dilation of $30 \pm 9 \%$ compared to controls (58 $\left.\pm 6 \%\right)$. On the other hand, umbilical vein endothelium-dependent dilation (Figure 7 B) was impaired only at the highest concentration of $\mathrm{ACh}\left(10^{-4}: 77 \pm 11 \%\right.$ vs. $\left.37 \pm 10 \%\right)$. These results suggest that gestational whole-body nano- $\mathrm{TiO}_{2}$ exposure impairs normal umbilical physiological vascular

endothelial function. Additionally, this deleterious effect seems to be more pronounced in the arterial circulation.

\section{Endothelium-Independent Dilation}

Endothelium-independent dilation of the umbilical artery was impaired in nano- $\mathrm{TiO}_{2}$ exposed animals at $10^{-7} \mathrm{M}(33 \pm 14 \%)$ and $10^{-6} \mathrm{M}(44 \pm 14 \%)$ of SNAP compared to controls (53 $\pm 14 \%$, $68 \pm 14 \%$ ) (Figure 8 A). In contrast to these findings, no point to point differences were seen in 
endothelium-independent dilation between control and nano- $\mathrm{TiO}_{2}$ exposed groups in the umbilical (Figure 8 B).

\section{Angiotensin II Sensitivity}

Both umbilical vessels were treated with increasing concentrations of ANGIl, a vasoactive agent that has been strongly implicated in pre-eclampsia and gestational hypertension [17] . The umbilical artery from nano- $\mathrm{TiO}_{2}$ exposed rats showed a remarkable increase in vasoconstriction $(-79 \pm 20 \%)$ compared to controls (-36 $\pm 10 \%)$ (Figure 9 A). Interestingly, no differences were seen in the response of the umbilical vein to ANGll between the Sham-control and nano- $\mathrm{TiO}_{2}$ exposed groups (Figure 9 B).

\section{Placental Immunohistochemistry}

Figure 10 A shows representative images of placental exposed sections stained with DAPI to visualize the nuclei of the cells present in the placenta. Co-staining of VWF and SMA was conducted to determine the localization of endothelial cells and to identify the blood vessels forming the placental microvasculature. An enlarged (40x) image of a placental microvessel is also shown (Figure 10 B). Immunohistochemistry of the placenta was conducted to: a) visualize the microvascular architecture in support of the hypothesis that the observed changes in outflow pressure may be ascribed to vasoactive responses, and b) verify the presence of the vascular endothelium. Future studies using this co-staining technique will be used to determine the impact of inhalation exposure on vascular density. 


\section{Discussion}

This is the first study to investigate the effects of gestational ENM inhalation exposure on placental hemodynamics. Herein, we also present a novel microvascular adaptation to study placental perfusion preparation [6]. The salient findings of the current work include that gestational nano$\mathrm{TiO}_{2}$ exposure results in an increase in placental vascular resistance as evidenced by the decrease in placental outflow pressure at baseline and following treatment with calcium-free superfusate (Figure $3 \mathbf{A}$ ) and (Figure 5). Treatment of placentas with the endothelium-dependent vasoactive agonist ACh increased average outflow pressure, indicating a decrease in vascular resistance of the placenta (Figure $3 \mathbf{B}$ ) and (Figure 6). Perhaps the most interesting finding based on our ex-vivo model is that placental and umbilical sensitivity to the vasoconstrictor ANGII is significantly augmented by gestational nano- $\mathrm{TiO}_{2}$ exposure (Figure 3 D) and (Figure 7 C). While the systemic microvascular effects of pulmonary ENM inhalation exposure have been previously reported by our group $[1,16,17]$ and others [27], the maternal-fetal vascular continuum remains severely understudied. Impairments in endothelium-dependent dilation in the uterine microcirculation [24] and umbilical vessels [28] caused by ENM exposure underscore the importance of filling this knowledge gap.

The ability of ENM to not only cross the fetoplacental barrier and affect placental function, but also to impair fetal development have previously been extensively reported [10]. Gestational inhalation exposure to $230 \mu \mathrm{g} / \mathrm{m}^{3}$ of cadmium oxide nanoparticles resulted in a decrease in the incidence of pregnancy by $23 \%$, diminished placental weight and delayed neonatal growth [3]. Additionally, intragastric administration of hydroxyl-modified single-walled carbon nanotubes in pregnant mice led to the development of skeletal defects, reduced ossification and morphological abnormalities [21]. Pulmonary exposure to nanosilica with a primary diameter of $70 \mathrm{~nm}$ caused significant fetal resorption and inhibited fetal growth [30]. $\mathrm{Nano}-\mathrm{TiO}_{2}$ inhalation exposure has also 
been shown to be able to migrate to the uterus and induce fetal resorption and negatively affect normal fetal development [19]. Furthermore, nano- $\mathrm{TiO}_{2}$ exposure is associated with a marked reduction in placental weight, fetal weight and number and fetal dysplasia [9].

The fetoplacental vasculature governs nutrient, gas and waste exchange and its characteristic low vascular resistance is essential for fetal health and conditions leading to an increase in placental resistance have been associated with IUGR and preeclampsia [14]. Because the placenta lacks autonomic innervation, vascular tone is dependent upon mechanical, autocrine, circulating vasoactive factors and changes in smooth muscle tone in the villous resistance vasculature. The umbilical artery and arterial tree is responsible for $57 \%$ of the total fetoplacental resistance, while $17 \%$ is attributable to the capillary bed and $26 \%$ to the venous tree and umbilical vein [22]. Therefore, any impairment in reactivity of the arterial and venous ends of the fetoplacental vasculature will significantly affect placental resistance and ultimately compromise developmental health.

Our results reflect that endothelium-dependent dilation in the maternal-fetal circulation is impaired by maternal ENM inhalation exposure. NO plays a particularly crucial role in maintaining the low basal tone of the maternofetal circulation, as reflected by NO synthase inhibitor infusion studies that increase placental perfusion pressure. It is germane to indicate that such an NO dependency most likely exists in the maternal microvascular circuit as NO signaling is not fully active in early microvascular development. This phenomenon is also observable in the current study where ACh treatment did not significantly increase placental outflow pressure vs the normal superfusate response (Figure 3 A vs 3 B). Other pathways that may be involved include the potent vasoactive 
prostanoids, but previous experiments have shown that infusion of the cyclooxygenase inhibitor indomethacin does not alter perfusion pressure of isolated placentas [14].

The renin-angiotensin system (RAS) is an important regulator of blood pressure as well as sodium and fluid homeostasis. The placenta possesses an autonomous, local RAS which has been associated with increased production of ANGII in IUGR and preeclampsia [23]. Within the placenta, most of the effects of ANGII, such as vasoconstriction, angiogenesis and cell growth are mediated by one of its cognate receptors, the AT1 receptor. Alternatively, the AT2 receptor reduces endothelial proliferation, migration, apoptosis and stimulates vasodilation. An imbalance between these two receptor populations in the maternal-fetal circulation resulting in an increased expression of AT1 receptors has been observed in preeclamptic placentas [15] and may explain the increase in sensitivity in nano- $\mathrm{TiO}_{2}$ exposed dams by both placentas and the umbilical artery observed in this work. Future studies are therefore required to determine the involvement of the maternal-fetal local RAS in the microvascular effects described herein.

There are several limitations to the ex-vivo perfused placental preparation adopted in this study that must be considered. First, deterioration and breakdown of the placental tissue occurs very rapidly once removed from the maternal environment. Therefore, this technique requires rapid mounting of the placental units onto the pressure myography chambers in order to minimize the deterioration of the tissue prior to and during experimentation. Second, measurements of the outflow pressure were conducted blindly without real-time visualization of the changes in the placental microcirculation. Lastly, the anatomical differences [8] between the human and rodent placentas indicate that caution should be exercised when extrapolating the results of the current study to humans. However, despite these differences, the vascular circuitry of the rodent placenta remains very representative of the human maternal-fetal circulation. Future studies, aimed at realtime visualization of the placental microvasculature and determination of the effect of ENM 
exposure on anatomical outcomes such as vascular density and structure, will be necessary to corroborate the present findings.

In conclusion, maternal ENM inhalation during gestation impairs fetoplacental vascular reactivity. Additionally, it appears that anatomic changes may also accompany this dysfunction. Because both may have profound impacts on fetal health, it is imperative to fully identify the underlying mechanisms. We have initially identified endothelium-dependent and -independent effects herein, as well as an increased sensitivity to ANGII. However, future studies further explore mechanisms of reactivity such as pressure and shear-dependent vascular responses. Of equal importance is to fully define the scope and depth of alterations in the fetoplacental vascular anatomy after maternal ENM inhalation. Finally, the most sensitive periods during gestational development for these toxicities should be determined. 
Funding information: We would like to acknowledge our financial support: R01-ES015022 (TRN), and NSF-1003907 (TRN and ABA).

Acknowledgements: We thank Kevin Engels from the West Virginia University (WVU) Department of Physiology and Pharmacology for his technical assistance in this study.

Disclaimer: The findings and conclusions in this report are those of the author(s) and do not necessarily represent the official position of the National Institute for Occupational Safety and Health, Centers for Disease Control and Prevention. 


\section{Figure Legends:}

Figure 1. Characterization of Nano- $\mathrm{TiO}_{2}$. (A) $\mathrm{SEM}$ image of nano- $\mathrm{TiO}_{2}$, (B) Energy dispersive spectroscopy showing elemental composition of the nano- $\mathrm{TiO}_{2}$ suspension used in this study, (C) Nano- $\mathrm{TiO}_{2}$ size distribution (mobility diameter, $190 \mathrm{~nm}$ ) using a scanning mobility particle sizer (SMPS), (D) Nano- $\mathrm{TiO}_{2}$ size distribution (aerodynamic diameter, $188 \mathrm{~nm}$ ) using a high resolution electrical low-pressure impactor (ELPI). (E) Total aerosol concentration $\left(10 \mathrm{mg} / \mathrm{m}^{3}\right)$ of engineered nano- $\mathrm{TiO}_{2}$ during maternal exposures.

Figure 2. Ex vivo placental perfusion preparation. (A) Umbilical artery (1); vein (2). (B) Input (arterial) perfusion pressure (1) $(0-80 \mathrm{~mm} \mathrm{Hg})$ is held constant while vasoactive agonists are added. Output pressure (2) and flow (3) ( $\mu \mathrm{l} / \mathrm{min})$ are variables responsive to placental resistance. Vascular diameter may be measured with video calipers. The chamber is designed for inverted microscopy, and fluorescent biomarkers may be easily added to the perfusate or superfusate.

Figure 3. Maternal nano- $\mathrm{TiO}_{2}$ inhalation exposure impairs endothelium-dependent placental hemodynamics. Mean outflow pressures were measured in sham-control and nano$\mathrm{TiO}_{2}$ exposed animals after the inflow pressure was increased in a stepwise manner and set at 0 mm Hg, 20 mm Hg, 40 mm Hg, 60 mm Hg and 80 mm Hg. Nano-TiO 2 inhalation exposure altered placental hemodynamics and decreased outflow (venous) pressure in placentas in normal superfusate $(A)$ and placentas treated with $A C h(B)(n=8) .{ }^{*}, P \leq 0.05$ sham-control group vs. nano- $\mathrm{TiO}_{2}$ exposed group.

Figure 4. Maternal nano- $\mathrm{TiO}_{2}$ inhalation exposure does not affect endotheliumindependent placental hemodynamics but increases ANGII sensitivity. Mean outflow 
pressures were measured in sham-control and nano- $\mathrm{TiO}_{2}$ exposed animals after the inflow pressure was increased in a stepwise manner and set at $0 \mathrm{~mm} \mathrm{Hg}, 20 \mathrm{~mm} \mathrm{Hg}, 40 \mathrm{~mm} \mathrm{Hg}, 60$ $\mathrm{mm} \mathrm{Hg}$ and $80 \mathrm{~mm} \mathrm{Hg}$. Maternal nano- $-\mathrm{TiO}_{2}$ inhalation exposure did not affect endothelialindependent placental hemodynamics $(A)$ but increased ANGII sensitivity $(B)(n=8) .{ }^{*}, P \leq 0.05$ sham-control group vs. nano- $\mathrm{TiO}_{2}$ exposed group.

Figure 6. $\mathrm{Nano}^{-\mathrm{TiO}_{2}}$ inhalation exposure and placental mean outflow pressure. Mean outflow pressure at $80 \mathrm{~mm} \mathrm{Hg}$ inflow pressure in both Sham-control and nano- $\mathrm{TiO}_{2}$ exposed groups and all treatment conditions is shown $(n=8)$. Statistics were analyzed with a student's ttest $(\mathrm{P} \leq 0.05),{ }^{*}$ Sham control group vs. nano- $\mathrm{TiO}_{2}$ exposed groups.

Figure 7. Maternal nano- $\mathrm{TiO}_{2}$ inhalation exposure impairs endothelium-dependent dilation of the umbilical artery and vein. (A) Endothelium-dependent dilation of the umbilical artery, (B) endothelium-dependent dilation of the umbilical vein from Sham-control and nano- $\mathrm{TiO}_{2}$ exposed animals was determined using pressure myography $(n=12-14)$. Statistics were analyzed with twoway ANOVA (P $\leq 0.05)$. * Sham control group vs. nano- $\mathrm{TiO}_{2}$ exposed group.

Figure 8. Maternal nano- $\mathrm{TiO}_{2}$ inhalation exposure impairs endothelium-independent dilation of the umbilical artery and vein. (A) Endothelium-independent dilation of the umbilical artery, (B) endothelium-independent dilation of the umbilical vein from Sham-control and nano$\mathrm{TiO}_{2}$ exposed animals was determined using pressure myography $(n=12-14)$. Statistics were analyzed with two-way ANOVA (P $\leq 0.05)$. ${ }^{*}$ Sham control group vs. nano- $\mathrm{TiO}_{2}$ exposed group.

Figure 9. Maternal nano- $\mathrm{TiO}_{2}$ inhalation exposure increases ANGll sensitivity of the umbilical artery. (A) ANGII dose-response curve of the umbilical artery (A) and vein (B) from 
Sham-control and nano- $\mathrm{TiO}_{2}$ exposed animals was determined using pressure myography $(\mathrm{n}=12-$ 14). Statistics were analyzed with two-way ANOVA ( $P \leq 0.05) .{ }^{*}$ Sham control group vs. nano$\mathrm{TiO}_{2}$ exposed group.

Figure 10. Placental immunohistochemistry. Smooth muscle actin is green, Von-Willebrand Factor is red, and DAPI nuclear staining is blue. (A) Representative image showing immunohistochemistry of a placental section from maternal nano-TiO2 exposed animal. The purpose was to identify the microvascular architecture and the structural components of the placenta. (B) An enlarged (40x) image of a placental microvessel displaying an intact endothelium (green). 


\section{Reference List:}

[1] Abukabda AB, Stapleton PA, Nurkiewicz TR. Metal Nanomaterial Toxicity Variations Within the Vascular System. Curr Environ Health Rep. 2016 Dec;3(4):379-391.

[2] Artis, D. and H. Spits, The biology of innate lymphoid cells. Nature, 2015. 517(7534): p. 293-301.

[3] Baenziger NL, Brodie GN, Majerus PW. A thrombin-sensitive protein of human platelet membranes. Proc Natl Acad Sci U S A. 1971. doi:10.1073/pnas.68.1.240

[4] Baisch, B.L., et al., Equivalent titanium dioxide nanoparticle deposition by intratracheal instillation and whole-body inhalation: the effect of dose rate on acute respiratory tract inflammation. Part Fibre Toxicol, 2014. 11: p. 5.

[5] Beamer, C.A., et al., IL-33 mediates multi-walled carbon nanotube (MWCNT)-induced airway hyper-reactivity via the mobilization of innate helper cells in the lung. Nanotoxicology, 2013. 7(6): p. 1070-81.

[6] Berne RM, Koeppen.B.M., Stanton BA. Berne \& Levy Physiology, Philadelphia, Mosby/Elsevier, 2010.

[7] Bonner JC, Silva RM, Taylor AJ, et al. Interlaboratory evaluation of rodent pulmonary responses to engineered nanomaterials: the NIEHS Nano GO Consortium. Environ Health Perspect 2013; $121676-82$

[8] Cassee FR, van Balen EC, Singh C, et al. Exposure, health and ecological effects review of engineered nanoscale cerium and cerium oxide associated with its use as a fuel additive. Crit Rev.Toxicol. 2011;41(3)213-229.

[9] Cat AND, Touyz RM. Cell signaling of angiotensin II on vascular tone: Novel mechanisms. Curr Hypertens Rep. 2011. doi:10.1007/s11906-011-0187-x 
[10] Champion JA, Walker A, Mitragotri S. Role of particle size in phagocytosis of polymeric microspheres. Pharm.Res. 2008;25(8)1815-1821.

[11] Courtois A, Andujar P, Ladeiro $\mathrm{Y}$, et al. Impairment of NO-dependent relaxation in intralobar pulmonary arteries: comparison of urban particulate matter and manufactured nanoparticles. Environ Health Perspect 2008; 116 1294-9

[12] Delmaschio A, Albors M, Evangelista V, et al. Measurement of Ionized Cytoplasmic Calcium Mobilization with the Photoprotein Aequorin in Human Polymorphonuclear Leukocytes Activated by Platelet Activating Factor (Paf). J Lipid Mediat. 1989.

[13] Dong M, Yan BP, Liao JK, Lam YY, Yip GWK, Yu CM. Rho-kinase inhibition: A novel therapeutic target for the treatment of cardiovascular diseases. Drug Discov Today. 2010. doi:10.1016/j.drudis.2010.06.011

[14] Eiserich JP, Baldus S, Brennan ML, et al. Myeloperoxidase, a leukocyte-derived vascular NO oxidase. Science (80- ). 2002. doi:10.1126/science.1106830

[15] Elder A, Gelein R, Silva V, et al. Translocation of inhaled ultrafine manganese oxide particles to the central nervous system. Environ Health Perspect. 2006;114(8):1172-1178. doi:10.1289/ehp.9030

[16] Forlow SB, Foley PL, Ley K. Severely reduced neutrophil adhesion and impaired host defense against fecal and commensal bacteria in CD18-/-P-selectin-/- double null mice. FASEB J 2002; 16 1488-96

[17] Fukata M, Kaibuchi K. Rho-family GTPases in cadherin-mediated cell-cell adhesion. Nat Rev Mol Cell Biol. 2001. doi:10.1038/35103068 
[18] G. C, N. C-M, E.E. K, et al. Thrombospondin-1 stimulates macrophage reactive oxygen species generation via CD47: A novel signaling event in atherosclerosis. Free Radic Biol Med. 2013.

[19] Grabrucker AM, Ruozi B, Belletti D, et al. Nanoparticle transport across the blood brain barrier. Tissue Barriers. 2016. doi:10.1080/21688370.2016.1153568

[20] Griendling KK, Ushio-Fukai M, Lassegue B, Alexander RW. Angiotensin II Signaling in Vascular Smooth Muscle: New Concepts. Hypertension. 1997. doi:10.1161/01.HYP.29.1.366

[21] Guilluy C, Brégeon J, Toumaniantz G, et al. The Rho exchange factor Arhgef1 mediates the effects of angiotensin II on vascular tone and blood pressure. Nat Med. 2010. doi:10.1038/nm.2079

[22] Guo H, Zhang J, Boudreau M, et al. Intravenous administration of silver nanoparticles causes organ toxicity through intracellular ROS-related loss of inter-endothelial junction. Part Fibre.Toxicol. 2016;1321.

[23] Hirano S, Fujitani Y, Furuyama A, et al. Uptake and cytotoxic effects of multi-walled carbon nanotubes in human bronchial epithelial cells. Toxicol.Appl.Pharmacol. 2010;249(1)8-15.

[24] Hougaard KS, Jackson P, Jensen KA, et al. Effects of prenatal exposure to surfacecoated nanosized titanium dioxide (UV-Titan). A study in mice. Part Fibre.Toxicol. 2010;716.

[25] Huang, J.P., et al., Nanoparticles can cross mouse placenta and induce trophoblast apoptosis. Placenta, 2015. 36(12): p. 1433-41.

[26] lii CAP, Verrier RL, Lovett EG, et al. Heart rate variability associated with particulate air pollution. :11-15. 
[27] Kan H, Wu Z, Lin Y-C, et al. The role of nodose ganglia in the regulation of cardiovascular function following pulmonary exposure to ultrafine titanium dioxide. Nanotoxicology. 2013:1-8. doi:10.3109/17435390.2013.796536

[28] Krebs, C., et al., Intrauterine growth restriction with absent end-diastolic flow velocity in the umbilical artery is associated with maldevelopment of the placental terminal villous tree. Am J Obstet Gynecol, 1996. 175(6): p. 1534-42.

[29] Learmont, J.G. and L. Poston, Nitric oxide is involved in flow-induced dilation of isolated human small fetoplacental arteries. Am J Obstet Gynecol, 1996. 174(2): p. 583-8.

[30] LeBlanc AJ, Moseley AM, Chen BT, et al. Nanoparticle inhalation impairs coronary microvascular reactivity via a local reactive oxygen species-dependent mechanism. Cardiovasc.Toxicol. 2010;10(1)27-36.

[31] Legramante JM, Valentini F, Magrini A, et al. Cardiac autonomic regulation after lung exposure to carbon nanotubes. Hum Exp Toxicol. 2009. doi:10.1177/0960327109105150

[32] Liu, X., et al., Anti-IL-33 antibody treatment inhibits airway inflammation in a murine model of allergic asthma. Biochem Biophys Res Commun, 2009. 386(1): p. 181-5.

[33] Mandler WK, Nurkiewicz TR, Porter DW, Olfert IM. Thrombospondin-1 mediates multiwalled carbon nanotube induced impairment of arteriolar dilation. Nanotoxicology. 2017. doi:10.1080/17435390.2016.1277275

[34] Mercer RR, Scabilloni JF, Hubbs AF, et al. Extrapulmonary transport of MWCNT following inhalation exposure. Part Fibre Toxicol 2013; 1038

[35] Michaudel, C., et al., Inflammasome, IL-1 and inflammation in ozone-induced lung injury. Am J Clin Exp Immunol, 2016. 5(1): p. 33-40. 
[36] Minarchick, V.C., et al., Cerium Dioxide Nanoparticle Exposure Improves Microvascular Dysfunction and Reduces Oxidative Stress in Spontaneously Hypertensive Rats. Front Physiol, 2015. 6: p. 339.

[37] Nemmar A, Delaunois A, Nemery B, et al. Inflammatory effect of intratracheal instillation of ultrafine particles in the rabbit: Role of C-fiber and mast cells. Toxicol Appl Pharmacol. 1999. doi:10.1006/taap.1999.8762

[38] Nurkiewicz TR, Porter DW, Barger M, et al. Systemic microvascular dysfunction and inflammation after pulmonary particulate matter exposure. Environ Health Perspect. 2006. doi:10.1289/ehp.8413

[39] Nurkiewicz TR, Porter DW, Hubbs AF, et al. Pulmonary nanoparticle exposure disrupts systemic microvascular nitric oxide signaling. Toxicol.Sci. 2009;110(1)191-203.

[40] Oberdorster G, Maynard A, Donaldson K, et al. Principles for characterizing the potential human health effects from exposure to nanomaterials: elements of a screening strategy. Part Fibre.Toxicol. 2005;28.

[41] Parivar, K., et al., The Effect of Nano-Titanium Dioxide on Limb Bud Development of NMRI Mouse Embryo In Vivo. Cell J, 2015. 17(2): p. 296-303.

[42] Pries AR, Secomb TW, Gaehtgens P. Biophysical aspects of blood flow in the microvasculature. Cardiovasc.Res. 1996;32(4)654-667.

[43] Pries AR, Secomb TW. Rheology of the microcirculation. Clin.Hemorheol.Microcirc. 2003;29(3-4)143-148.

[44] Rajagopalan S, Brook RD. The Indoor-Outdoor Air-Pollution Continuum and the Burden of Cardiovascular Disease: An Opportunity For Improving Global Health. Glob.Heart 2012;7(3)207-213. 
[45] Rajagopalan S, Kurz S, Münzel T, et al. Angiotensin II-mediated hypertension in the rat increases vascular superoxide production via membrane NADH/NADPH oxidase activation: Contribution to alterations of vasomotor tone. J Clin Invest. 1996. doi:10.1172/JCI118623

[46] Schwartzkopff B, Mundhenke M, Strauer BE. Alterations of the architecture of subendocardial arterioles in patients with hypertrophic cardiomyopathy and impaired coronary vasodilator reserve: a possible cause for myocardial ischemia. J Am Coll Cardiol 1998; 31 108996.

[47] Shah, D.M., Role of the renin-angiotensin system in the pathogenesis of preeclampsia. Am J Physiol Renal Physiol, 2005. 288(4): p. F614-25.

[48] Spits, H., et al., Innate lymphoid cells--a proposal for uniform nomenclature. Nat Rev Immunol, 2013. 13(2): p. 145-9.

[49] Stapleton PA, Abukabda AB, Hardy SL, Nurkiewicz TR. Xenobiotic pulmonary exposure and systemic cardiovascular response via neurological links. Am J Physiol Circ Physiol. 2015. doi:10.1152/ajpheart.00546.2015

[50] Stapleton PA, Nurkiewicz TR. Vascular distribution of nanomaterials. Wiley.Interdiscip.Rev.Nanomed.Nanobiotechnol. 2014;6(4)338-348.

[51] Stapleton, P.A. and T.R. Nurkiewicz, Maternal nanomaterial exposure: a double threat to maternal uterine health and fetal development? Nanomedicine (Lond), 2014. 9(7): p. 929-31.

[52] Thompson LC VA. Vascular Tissue Contractility Changes Following Late Gestational Exposure to Multi-Walled Carbon Nanotubes or their Dispersing Vehicle in Sprague Dawley Rats. J Nanomed Nanotechnol. 2014. doi:10.4172/2157-7439.1000201 
[53] Trickler WJ, Lantz SM, Murdock RC, et al. Silver nanoparticle induced blood-brain barrier inflammation and increased permeability in primary rat brain microvessel endothelial cells. Toxicol.Sci. 2010;118(1)160-170.

[54] Wang J, Byrne JD, Napier ME, et al. More effective nanomedicines through particle design. Small 2011;7(14)1919-1931.

[55] Yamashita, K. and Y. Yoshioka, [Safety assessment of nanomaterials in reproductive developmental field]. Yakugaku Zasshi, 2012. 132(3): p. 331-5.

[56] Yang, Q., et al., Group 2 innate lymphoid cells mediate ozone-induced airway inflammation and hyperresponsiveness in mice. J Allergy Clin Immunol, 2016. 137(2): p. 571-8.

[57] Zoltowska, A.M., et al., The interleukin-33 receptor ST2 is important for the development of peripheral airway hyperresponsiveness and inflammation in a house dust mite mouse model of asthma. Clin Exp Allergy, 2016. 46(3): p. 479-489 


\section{Table 1: Animal Characteristics}

\begin{tabular}{|c|c|c|c|c|}
\hline & $\mathrm{N}$ & Age (weeks) & Weight (grams) & Number of Pups \\
\hline Control & 8 & $12 \pm 2$ & $310 \pm 27$ & $6 \pm 2$ \\
\hline Exposed & 10 & $13 \pm 1$ & $344 \pm 16$ & $9 \pm 3$ \\
\hline
\end{tabular}




\section{Table 2: Umbilical Artery Characteristics}

\begin{tabular}{|c|c|c|c|c|c|c|c|}
\hline & $\mathrm{n}$ & $\begin{array}{c}\text { Inner } \\
\text { diameter } \\
(\mu \mathrm{m})\end{array}$ & $\begin{array}{c}\text { Outer } \\
\text { diameter } \\
(\mu \mathrm{m})\end{array}$ & $\begin{array}{c}\text { Tone } \\
(\%)\end{array}$ & $\begin{array}{c}\text { Passive } \\
\text { diameter inner } \\
(\mu \mathrm{m})\end{array}$ & $\begin{array}{c}\text { Passive diameter } \\
\text { outer }(\mu \mathrm{m})\end{array}$ & $\begin{array}{c}\text { Wall Tension } \\
(\text { Newton/meter })\end{array}$ \\
\hline Control & 14 & $402 \pm 11$ & $664 \pm 13$ & $15 \pm 4$ & $459 \pm 15$ & $707 \pm 17$ & $0.34 \pm 0.1$ \\
\hline Exposed & 12 & $398 \pm 5$ & $652 \pm 14$ & $17 \pm 7$ & $442 \pm 9$ & $699 \pm 15$ & $0.37 \pm 0.2$ \\
\hline
\end{tabular}




\section{Table 3: Umbilical Vein Characteristics}

\begin{tabular}{|c|c|c|c|c|c|c|c|}
\hline & $\mathrm{n}$ & $\begin{array}{c}\text { Inner } \\
\text { diameter } \\
(\mu \mathrm{m})\end{array}$ & $\begin{array}{c}\text { Outer } \\
\text { diameter } \\
(\mu \mathrm{m})\end{array}$ & $\begin{array}{c}\text { Tone } \\
(\%)\end{array}$ & $\begin{array}{c}\text { Passive } \\
\text { diameter inner } \\
(\mu \mathrm{m})\end{array}$ & $\begin{array}{c}\text { Passive diameter } \\
\text { outer }(\mu \mathrm{m})\end{array}$ & $\begin{array}{c}\text { Wall Tension } \\
(\text { Newton/meter })\end{array}$ \\
\hline Control & 10 & $458 \pm 30$ & $718 \pm 38$ & $14 \pm 1$ & $543 \pm 29$ & $753 \pm 38$ & $0.32 \pm 0.2$ \\
\hline Exposed & 8 & $447 \pm 16$ & $689 \pm 14$ & $12 \pm 3$ & $623 \pm 40$ & $741 \pm 17$ & $0.43 \pm 0.4$ \\
\hline
\end{tabular}


Figure 1:

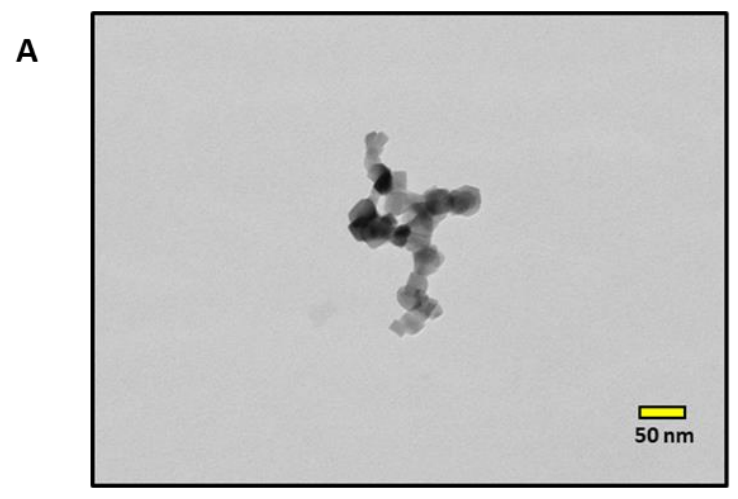

B

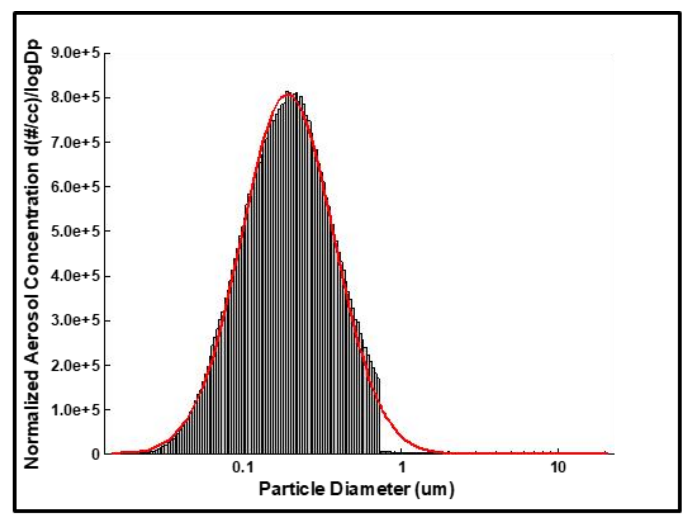

C

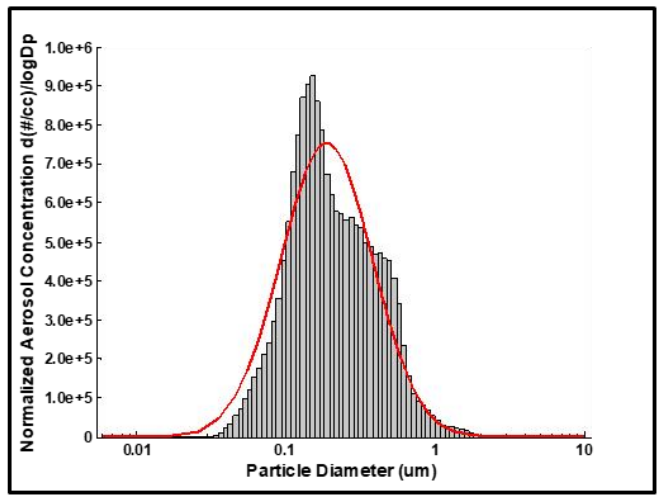

D

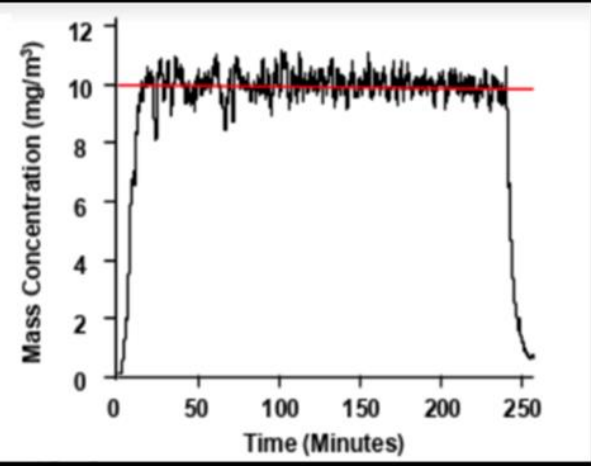


Figure 2:

B
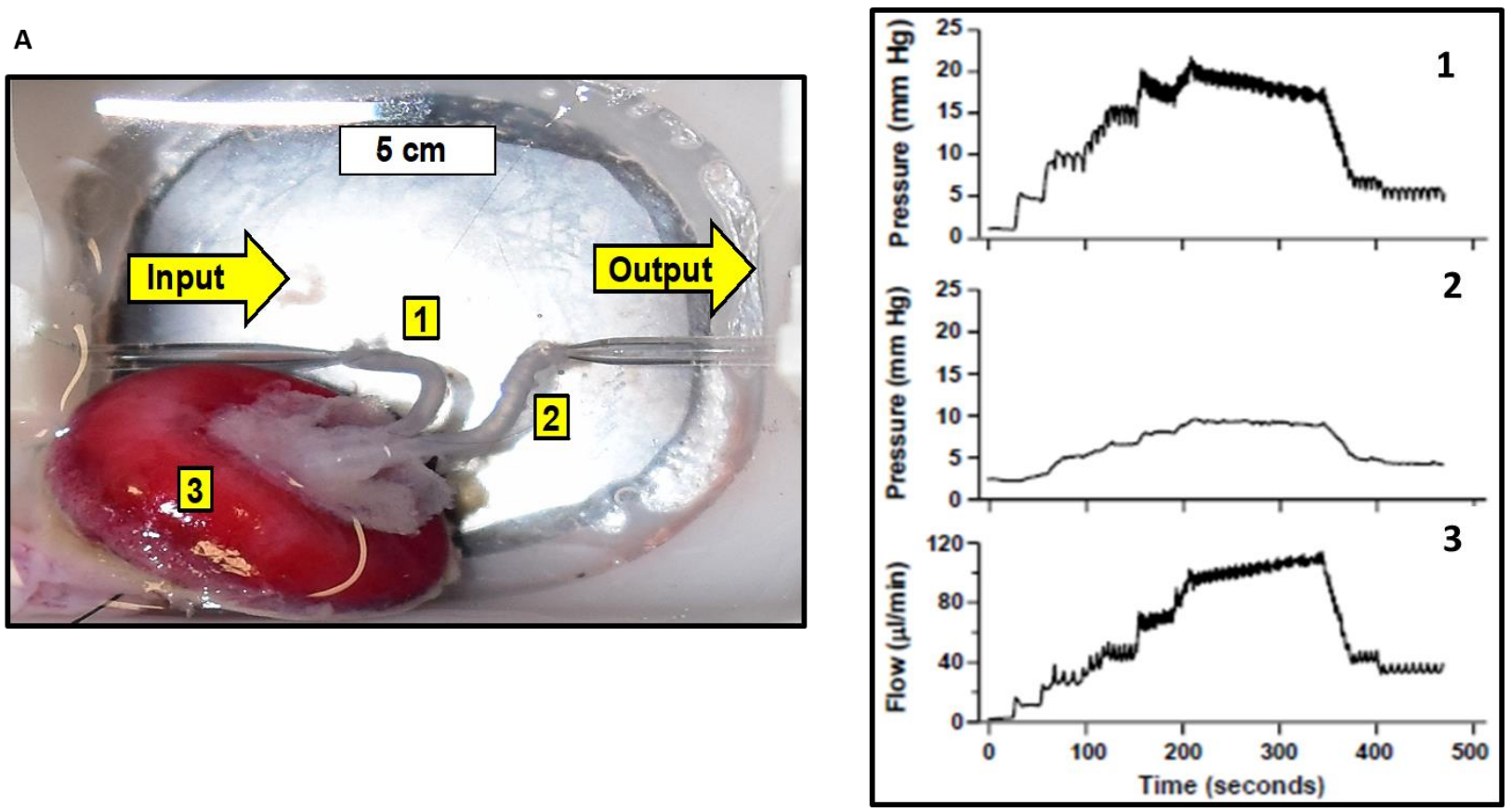
Figure 3:

A

Normal Superfusate

B
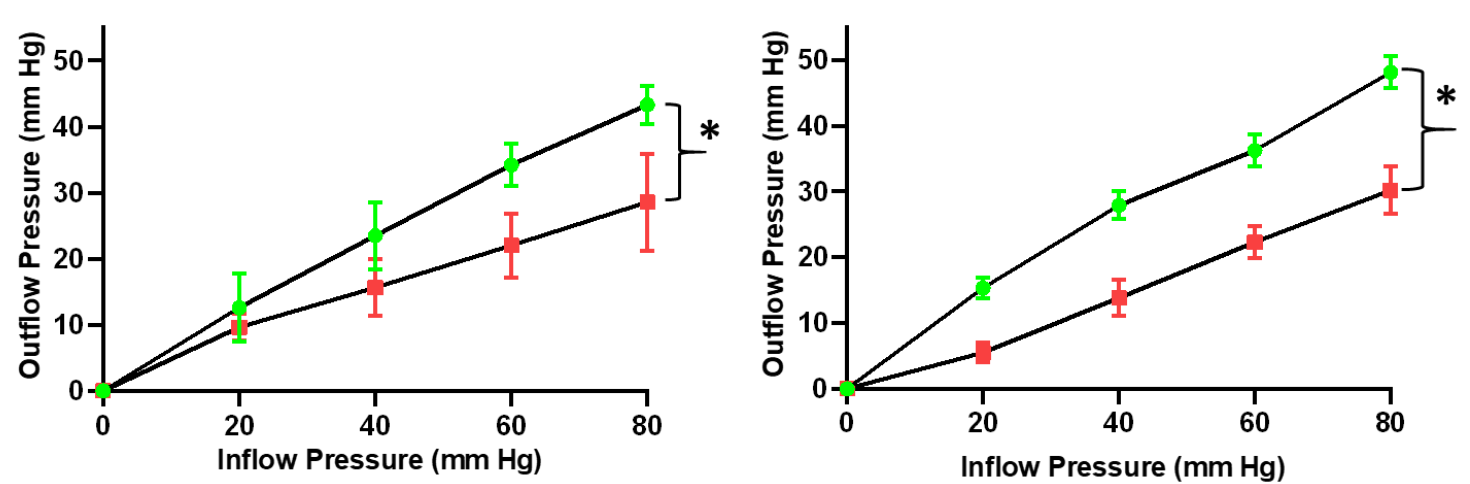

- - Sham-Control
- - Nano- $\mathrm{TiO}_{2}$ Exposed
$* \mathrm{P}<0.05$


Figure 4:

A

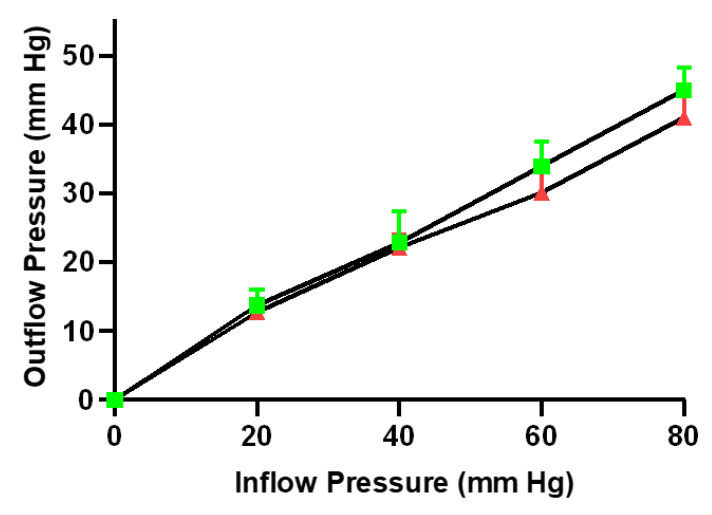

B

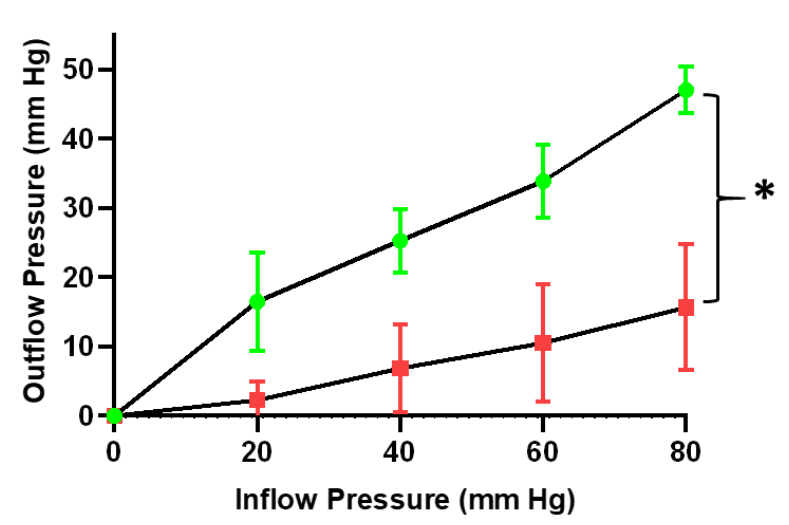

$$
\begin{aligned}
& -\sim \text { Sham-Control } \\
& - \text { - Nano- } \mathrm{TiO}_{2} \text { Exposed } \\
& \text { *P<0.05 }
\end{aligned}
$$


Figure 5:

\section{Calcium-Free Superfusate}

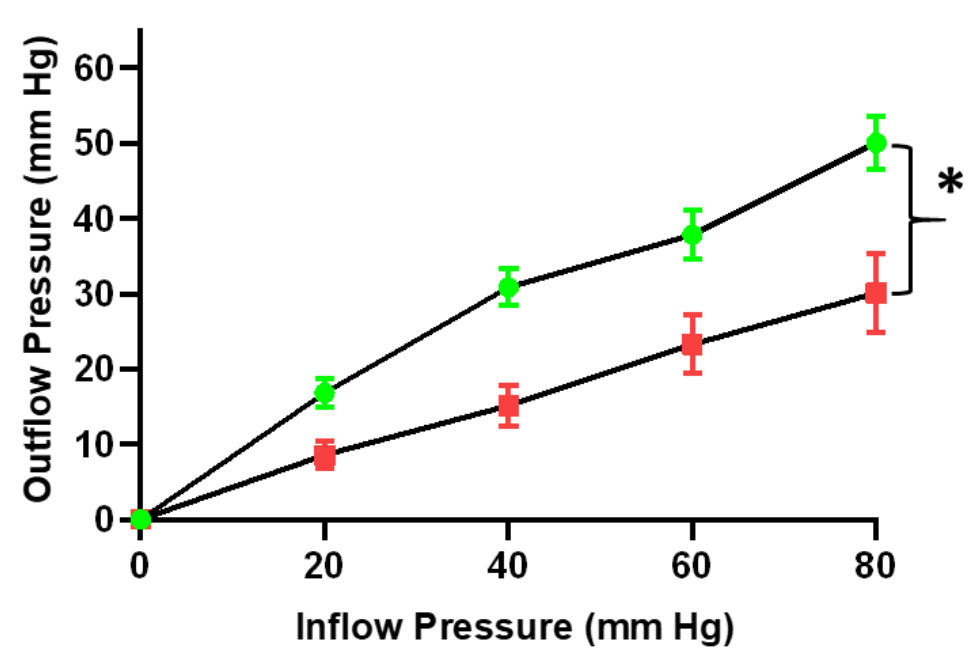


Figure 6:

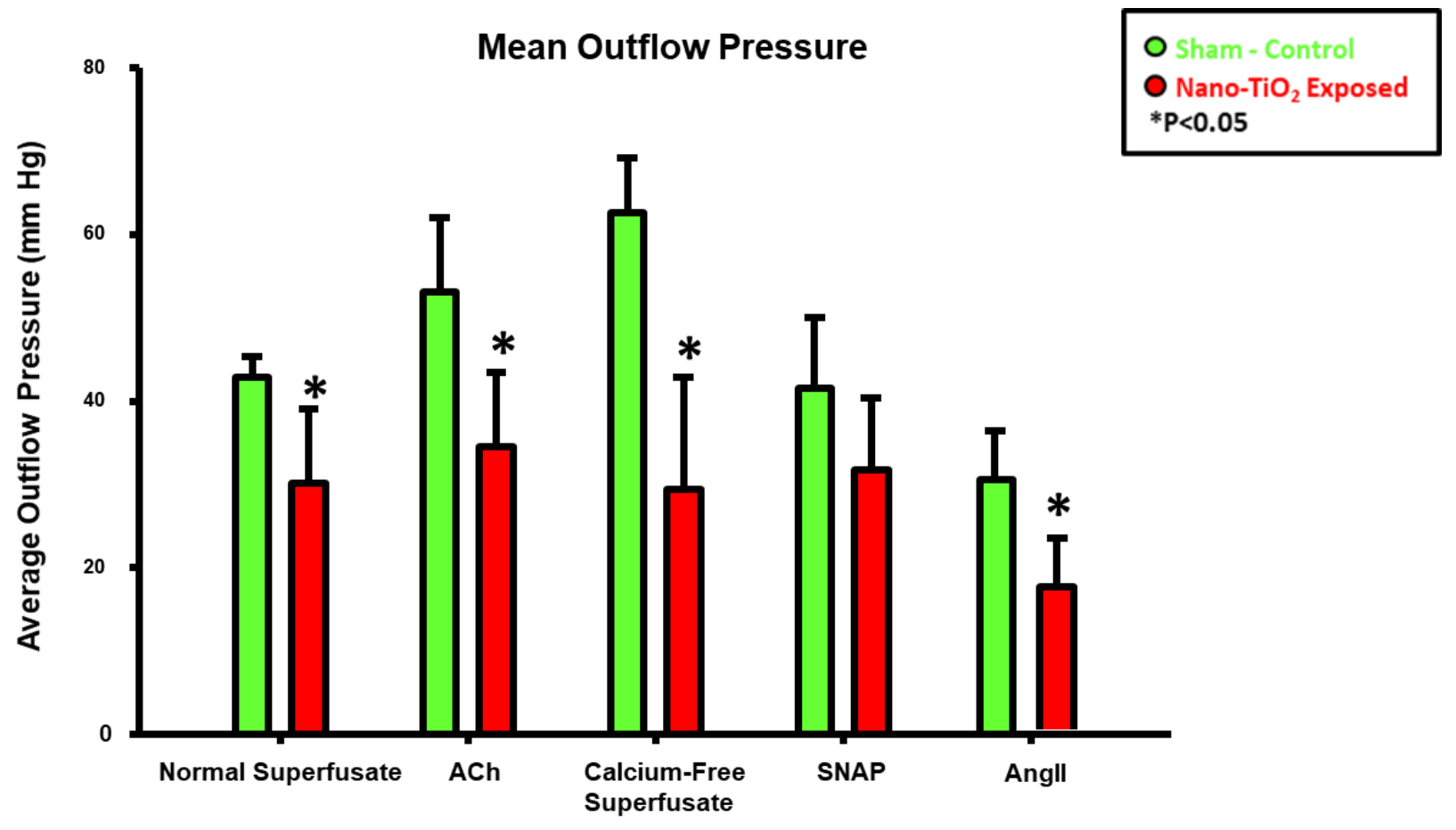


Figure 7:

A Endothelium-Dependent Dilation Umbilical Artery

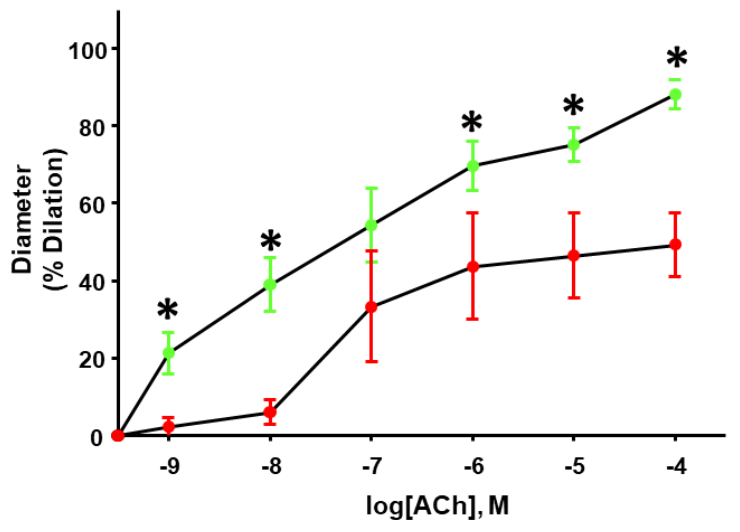

O Sham - Control

O Nano-TiO ${ }_{2}$ Exposed ${ }^{*} \mathrm{P}<0.05$
B

Endothelium-Dependent Dilation Umbilical Vein

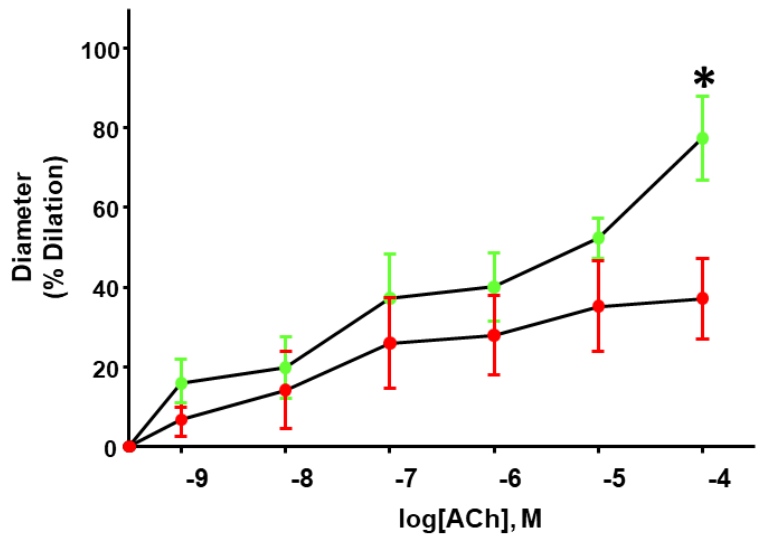


Figure 8:
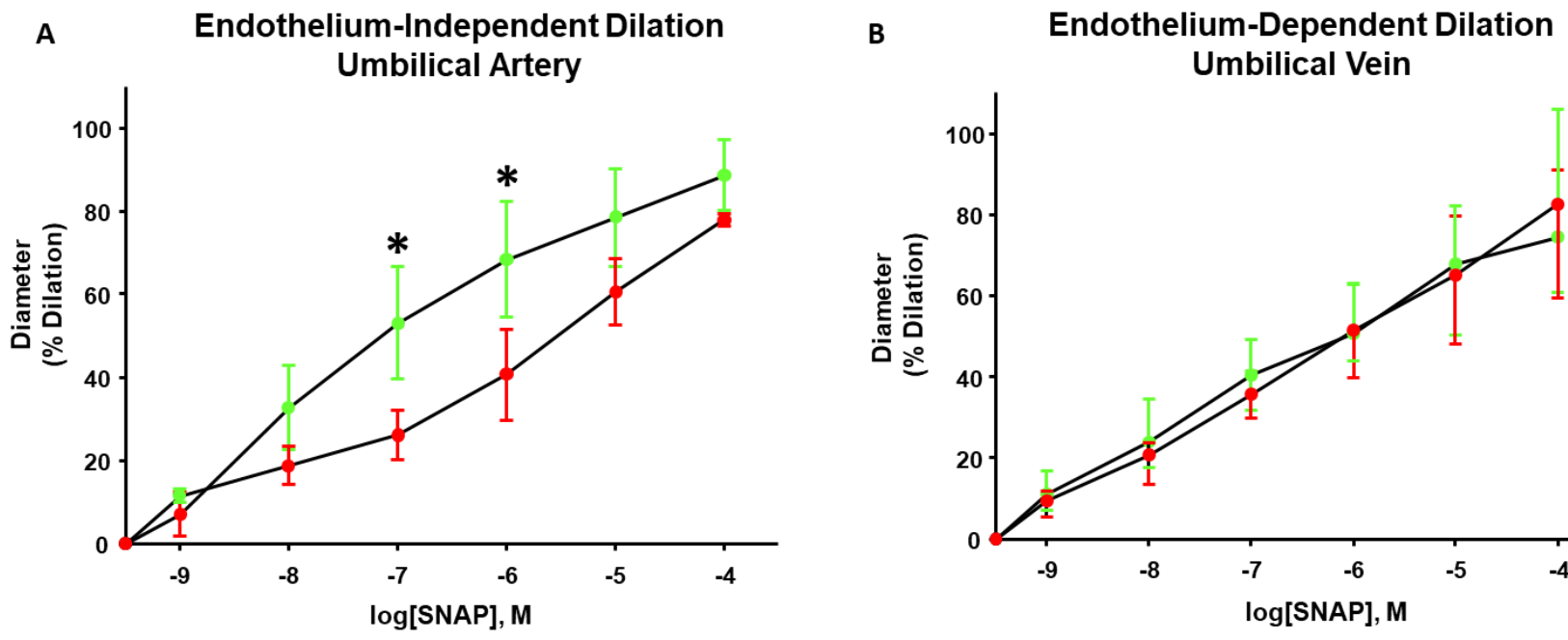

O Sham - Control

O Nano-TiO 2 Exposed *P<0.05 
Figure 9:

A

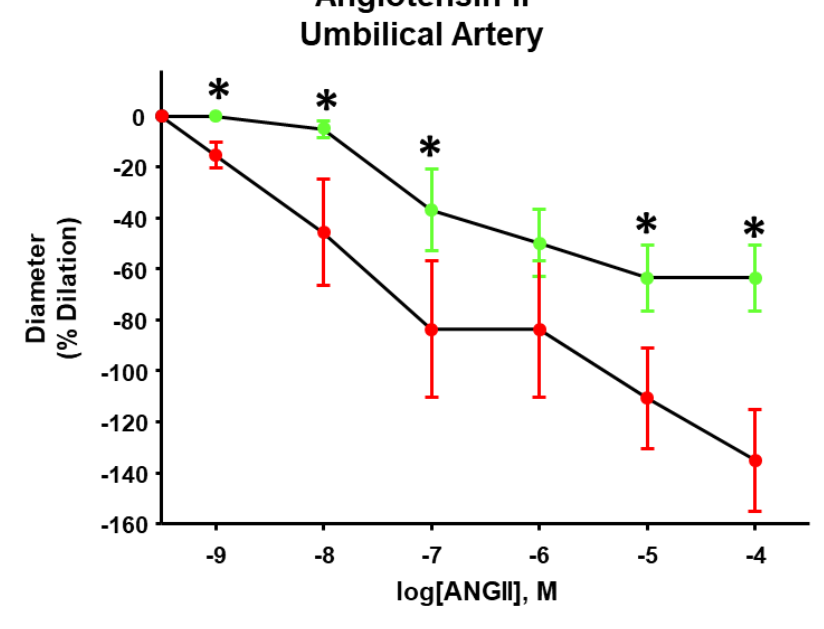

O Sham - Control

O Nano-TiO ${ }_{2}$ Exposed $* \mathrm{P}<0.05$
B

Angiotensin II

Umbilical Vein

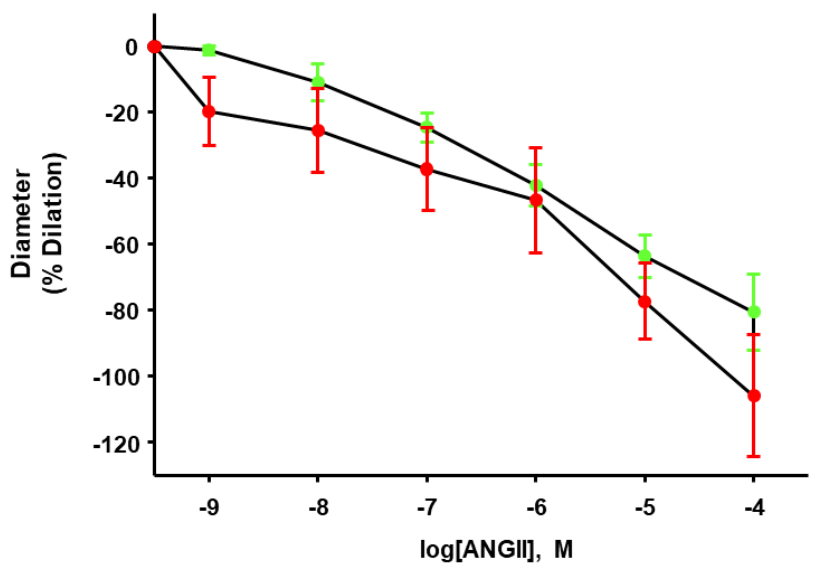


Figure 10:
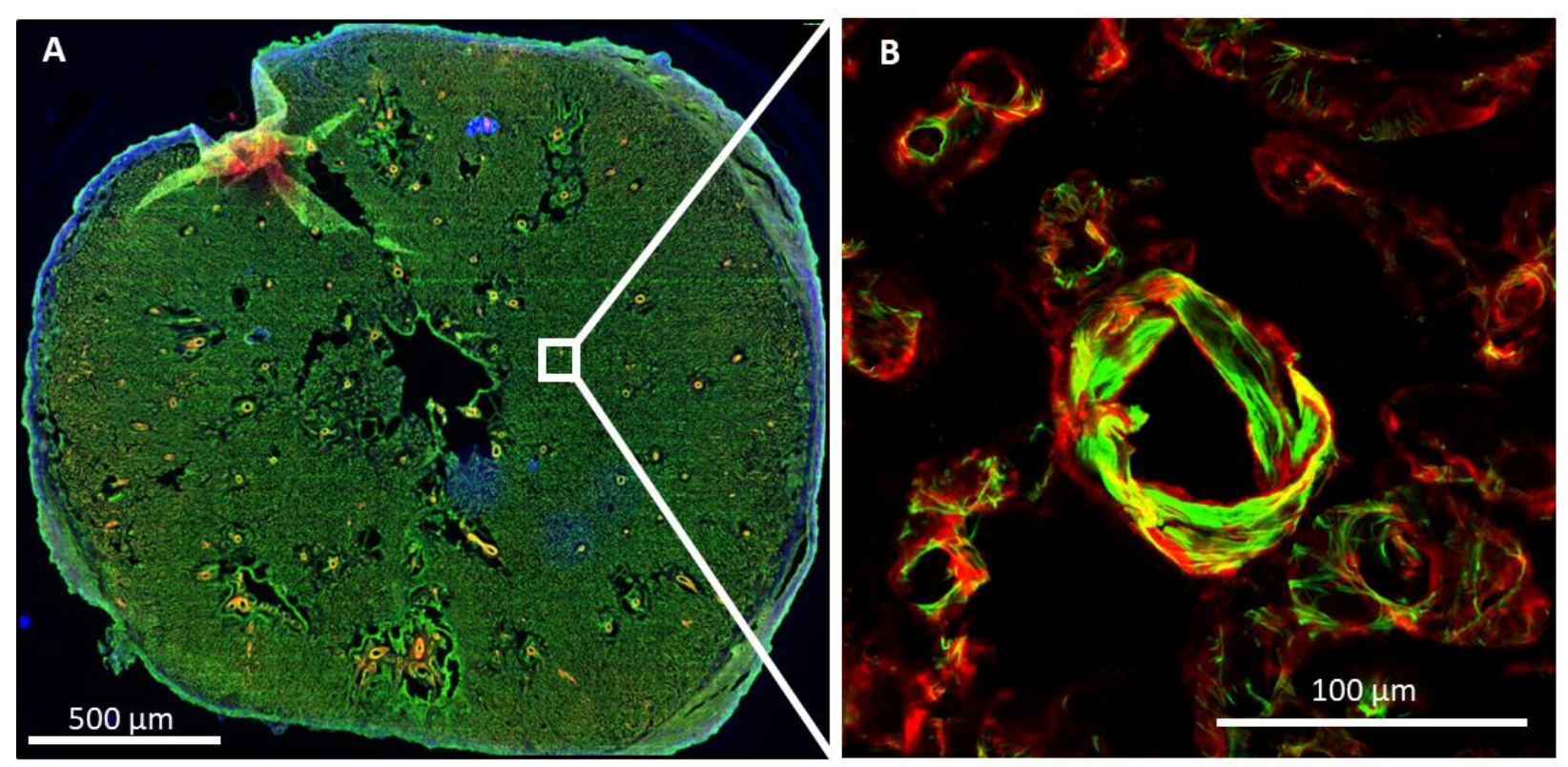


\section{General Discussion}

The primary objective of this dissertation project was to determine the effects of acute pulmonary ENM exposure on the microcirculation and identify the potential mechanisms resulting in vascular dysfunction. Overall, this dissertation project can be divided into 3 distinct components. The first part maps the effects of ENM exposure on vascular reactivity along the vascular tree (i.e. from the macrocirculation to the microcirculation) and identifies the most sensitive segments to pulmonary ENM insult by assessing endothelium-dependent, endothelium-independent and adrenergic responsiveness. The second study identifies the involvement of innate lymphoid cells in the acute inflammatory response to nano- $\mathrm{TiO}_{2}$. Lastly, the third and final component of this work assess the effect of maternal gestational inhalation exposure to nano- $\mathrm{TiO}_{2}$ on placental vascular resistance and umbilical vascular reactivity.

\section{A) Vascular Heterogeneity and Microvascular Sensitivity}

The microcirculation is crucial for the generation of the total peripheral resistance necessary for adequate tissue perfusion and nutrient-waste exchange and unresolved impairments in its function may contribute to the development of several systemic conditions such as diabetes and hypertension [46]. Notwithstanding the prominent role played by the microvasculature in the tissue perfusion, the mechanisms through which ENM inhalation exposure disrupts normal vascular function remain to be clarified. In general, all the studies indicated that pulmonary ENM exposure causes endothelium-dependent dysfunction in the microvasculature.

Study 1 first identified and directly compared the differential effect of ENM exposure on discrete anatomical segments of the vascular tree in different vascular beds. Acute pulmonary ENM

exposure was associated with microvascular and macrovascular dysfunction caused by an 
impairment in endothelium-dependent and endothelium-independent dilation. Lastly, the dysfunction observed at the microvascular level was the most robust, underscoring the sensitivity of the microcirculation to acute pulmonary ENM exposure. In this series of experiments, nano$\mathrm{TiO}_{2}$ exposure was found to significantly decrease endothelium-dependent relaxation in the thoracic aorta, the femoral arteries and third-order mesenteric arterioles. Furthermore, what is undoubtedly most compelling is the finding that exposure to high concentrations of nano- $\mathrm{TiO}_{2}$ almost completely abolished endothelium-dependent dilation and acetylcholine sensitivity in $4^{\text {th }}$ and $5^{\text {th }}$ order mesenteric arterioles. It is also worth noting that a dose-independent effect of nano$\mathrm{TiO}_{2}$ was seen on the reactivity of the thoracic aorta. Indeed, the lowest dose of nano- $\mathrm{TiO}_{2}(20$ $\mu \mathrm{g})$ induced the greatest endothelium-dependent dysfunction, while the intermediate dose (100 $\mu \mathrm{g})$ caused the most robust decrease in endothelium-independent responsiveness. Additionally, nano- $\mathrm{TiO}_{2}$ exposure significantly decreased the mean inner diameter and the tone of the $4^{\text {th }}$ and $5^{\text {th }}$ mesenteric arterioles. Studies 2 and 3 confirmed the systemic vascular effects of nano- $\mathrm{TiO}_{2}$, indicating that the observed impairments were independent of the vascular bed and exposure technique used (whole-body inhalation vs. instillation).

Impairments in the endothelium-dependent dilation following ENM exposure have been extensively reviewed [1]. Indeed, microvascular function has been shown to be impaired in the coronary [30], uterine [51], mesentery [36], and pulmonary [11] microvasculature following particulate matter exposure.

The endothelial layer is composed of receptor-effector cells capable of sensing different physical or chemical intravascular stimuli. These cells then produce a large array of paracrine, endocrine and autocrine agonists that counteract the effect of these stimuli and maintain homeostasis. Nitric 
oxide (NO) is perhaps the most ubiquitous and most studied vasodilator produced by endothelial cells. NO is a biologically active, lipophilic gas capable of diffusing easily across membranes and of crossing the endothelial intima and reaching the vascular smooth muscle tissue, subsequently stimulating the degradation of guanosine triphosphate (GTP) to release cyclic guanosine monophosphate cGMP, which in turn is responsible for vascular smooth muscle relaxation and vasodilation [6].

NO is produced by the enzymatic action of nitric oxide synthase (NOS) on the amino acid Larginine, which results in the production of $\mathrm{NO}$ and L-citrulline. For this transamination reaction to occur, $\mathrm{O}_{2}$ and the coenzyme nicotinamide adenine dinucleotide phosphate must be present. Cofactors such as tetrahydrobiopterine $\left(\mathrm{BH}_{4}\right)$ and flavine-adenine dinucleotide (FAD) [6]. Endogenous vascular NO production is severely compromised after ENM exposure [39] as a result of oxidative stress, inflammation and/or direct particle interaction with the tissues. Oxidant products such as the superoxide anion, hydrogen peroxide, the hydroxyl radical, hypochlorous acid and lipid radicals are normally produced during aerobic metabolism. The resulting molecules are extremely reactive and are known as Reactive Oxygen species (ROS). Under normal conditions, ROS generation is controlled by the presence of antioxidants and enzymes including superoxide dismutase, glutathione peroxidase and catalase which are responsible for the conversion of ROS to oxygen and water. Following ENM exposure, the intracellular levels of ROS have been shown to drastically increase resulting in oxidative stress [36]. Excess of ROS may lead to the oxidation of $\mathrm{NO}$ leading to the formation of peroxynitrite (ONOO), an extremely reactive molecule that can result in more oxidation. In this way, intravascular NO concentrations may significantly decrease, thus affecting their vasodilatory responses. 
The most important stimulus for the basal release of NO by endothelial cells is shear stress [29]. The hemodynamic conditions within the vasculature lead to the development of superficial stress on the vessel walls due to blood flow which is applied mainly to the endothelial layer. Increases in blood flow velocity lead to an increase in shear stress and a vasodilation proportional to the amount of NO released by the endothelium $[42,43]$. This NO mediated response, otherwise known as endothelium-dependent dilation, is mediated by specialized ion channels, such as $\mathrm{Ca}^{2+}$ activated $\mathrm{K}^{+}$channels, that open in response to changes in shear stress [42], causing hyperpolarization of the endothelial cells, the influx of $\mathrm{Ca}^{2+}$ and the generation of $\mathrm{NO}$ [6].

The observed endothelium-dependent dysfunction may have also been induced by the local deposition of hemoproteins [13]. Myeloperoxidase, a microbicidal molecule secreted by leukocytes, has been shown to deposit around vascular endothelial cells in response to ENM exposure [38], resulting in an impaired endothelium-dependent response. Altered vascular responsiveness has been associated with catalytic consumption of NO by the substrate radicals generated (hypochlorous acid). Decreased NO generation and microvascular dysfunction have also been observed following MWCNT exposure and linked to the activation of the scavenger receptor CD47 [33]. CD47 signaling is activated by thrombospondin-1 (TSP-1), a primary component of the a-granules of platelets [34, causing activation of NADPH oxidase-1 (NOX-1) in endothelial cells and leukocytes [45]. Activation of NOX-1 results in ROS generation and subsequent leukocyte adhesion, endothelial disorganization and permeability [12], which may further promote endothelial dysfunction.

The decreased endothelium-independent dilation observed in study 1 may have been caused by alterations in VSM signaling. ENM exposure may result in changes in calcium handling or an 
increase in phosphodiesterase activity - both of which may reduce the sensitivity to the vasodilatory effect of $\mathrm{NO}$ [11]. Changes in vascular contractility may also occur due to alterations in RhoA signaling [52]. The RhoA-Rho kinase pathway may contribute to an increase in the contractile response in VSM via regulation of intracellular calcium and phosphorylation of myosin light chain phosphatase [17]. Indeed, an increase in Rho-kinase activity has been shown to be associated with increased responsiveness to PE and endothelin-1 in uterine and aortic segments following intravenous exposure to fullerenes [52]. Furthermore, up-regulation of the Rho-kinase pathway has also been linked to decreased expression and activity of eNOS [13], which may reduce relaxation and enhance vascular contraction.

\section{B) Potential Mechanisms}

Historically, epidemiological studies have extensively confirmed a causal relationship between particulate matter exposure and cardiovascular morbidity and mortality [44], and 3 generalized intermediary biological pathways and specific biological responses have been implicated; direct tissue-particle interaction, systemic oxidative stress and inflammation and dysregulation of the autonomic nervous system [44].

The inflammatory hypothesis, which states that pulmonary ENM exposure to particulate matter initiates a systemic inflammatory response, is undoubtedly the most studied. The immune response to ENM exposure involves both the innate and adaptive branches [7]. Within the literature (for example [7]), studies have shown that the innate immune system plays a central role in the host response against inhaled particles and fibers [10]. Most inhaled ENM deposited in the alveolar regions are phagocytized by alveolar macrophages [7]. Individual ENM may, 
however, escape phagocytosis and interact directly with pulmonary epithelial cells [4]. ENM taken up by macrophages are cleared from the lungs via the mucociliary escalator and the lymphatic system [7]. High aspect ratio ENM (CNT, nanofibers, nanowires), nano- $\mathrm{TiO}_{2}$, or the formation of bridges by CNT bundles may disrupt macrophage clearance, leading to cytokine secretion [55]. $\mathrm{CeO}_{2}-\mathrm{NP}$ have been reported to impair peripheral monocyte function by inducing mitochondrial damage and overexpression of the apoptosis-inducing factor, leading to increased autophagy [8]. High-aspect ratio ENM activate the inflammasome in alveolar macrophages and trigger the secretion, via disruption of lysosomal integrity and ROS, of the proinflammatory cytokines IL-1 $1 \beta$ and IL-18 [34]. Ag-NP [22] and MWCNT [23] increase the expression of NF-KB and cyclooxygenase-2 (COX-2) in RAW264.7 macrophages in vitro. These inflammatory mediators may trigger a systemic inflammatory cascade by activating other immune effector cells including innate lymphoid cells.

The most conspicuous knowledge gap is the one linking these immune effects with the systemic microvascular dysfunction. For this reason, the main objective of study 2 was to study the potential mechanism linking acute pulmonary ENM exposure and the observed microvascular impairments. The salient findings of study 2 are that acute pulmonary nano- $\mathrm{TiO}_{2}$ causes epithelial cell injury, as evidenced by the increased pulmonary levels of the epithelial "alarmin" IL-33. Additionally, nano- $\mathrm{TiO}_{2}$ instillation increased BALF and circulating levels of the $\mathrm{TH}_{2}$ cytokines IL4, IL-5 and IL-13 after only 2 hours. The impairment in endothelial-dependent dilation observed in the uterine microcirculation was partially improved by pre-treatment with an antibody specific for IL-33. The finding that ENM exposure increases lung IL-33 levels has been previously reported in other studies with MWCNT [5]. It is known that canonically-activated naive Th2 cells require prolonged continuous stimulation by antigen-presenting cells [2]. Therefore, we investigated the potential role of innate lymphoid cells (ILC1 and ILC2), which have been already implicated in 
asthmatic responses to environmental allergens [56] and acute lung injury [35]. Interestingly, nano- $\mathrm{TiO}_{2}$ increased ILC2 but not ILC1 levels within the lungs as early as 4 hours post-exposure. These results point to the shifting of the innate immune response to ENM towards a non-canonical Th2 or allergic phenotype [56]. Moreover, ILC2 are the dominant population of innate lymphoid effector cells in the lungs and are capable of secreting large amounts of $\mathrm{TH}_{2}$ cytokines in response to specific stimuli [2]. In mice, an IL-25 inducible, lineage negative cell population was shown to secrete large amounts of IL-5 and IL-13 [56]. In humans, a CD34+ cell population expressing the receptor for IL-33 (IL-33R) was identified and shown to secrete Th2 cytokines upon treatment with IL-33 [56]. The location of ILC2 at barrier sites and mucosal tissue [2] is especially important, especially in the lung where they have been associated with airway hyper-reactivity on exposure to house dust mites [56] and papain [32]. Interestingly, airway hyper-reactivity and increased airway resistance have also been reported following ENM inhalation exposure [5]. In mice, MWCNT exacerbate allergic airway inflammation following ovalbumin sensitization [5].

ENM inhalation may lead to the release within the lungs of alarm signals or damage associated molecular patterns (DAMPs) such as IL-33 directly into the lung microenvironment and into the circulation. These DAMPs may then acutely stimulate both the proliferation of lung-resident ILC2 and the migration of circulating ILC2 to the lungs. Once stimulated, ILC2 secrete significant quantities of the cytokines IL-4, IL-5 and IL-13. IL-5 has been shown to be necessary for eosinophil homeostasis and B cell function [2]. In addition, induction of B cell proliferation and secretion of $\lg A$, $\lg M$, IgE and $\lg G 1$ have also been shown in vitro and in vivo [2]. Further, IL-4 and IL-13 have the potential of targeting non-immune and immune cells present in the lungs. These mediators initiate mucus secretion, goblet hyperplasia, priming of macrophages, dendritic cells and neutrophils [48]. Therefore, ENM likely trigger the release of soluble cytokines from the lungs since evidence of translocation from the lung is scarce [40]. Nonetheless, it is possible that 
a small proportion of the inhaled ENM will translocate from the lung to distant organs and tissues and impair their function by directly disrupting tissue barriers [34], catalyzing Fenton-reactions and potentially inducing local oxidative stress.

The proinflammatory cytokines and chemokines secreted into the circulation may then activate circulating neutrophils and vascular endothelial cells [16]. Studies by our group have identified acute increases in neutrophil rolling and adhesion following inhalation exposure to nano- $\mathrm{TiO}_{2}$ in uterine venules [51] and to $\mathrm{CeO}_{2}-\mathrm{NP}$ in the mesenteric microcirculation [36]. Therefore, it is likely that pulmonary ENM exposure promotes leukocyte tethering, rolling and adhesion. Further studies are needed to confirm the role of ILC2 in the acute response to pulmonary ENM exposure and the link between Th2 mediators and leukocyte/endothelium activation.

The inflammatory and direct-tissue interaction hypotheses cannot entirely account for the vascular dysfunction associated with pulmonary ENM exposure. Indeed, increasing evidence suggests that PM (including ENM) may interact with the central nervous system and/or stimulate an autonomic arc between the lung and the systemic tissue [49]. For example, ultrafine particulate matter has been shown to induce a pulmonary inflammatory response resulting in histamine release from unmyelinated $\mathrm{C}$ fibers and mast cells [37]. Stimulation of these vagal afferent neurons may lead to dysregulation of autonomic signaling. Furthermore, intratracheal instillation of $1 \mathrm{mg} / \mathrm{ml}$ of SWCNT altered baroreflex function in Wistar-Kyoto rats, thus affecting the autonomic control of cardiovascular function [27]. Potential central pathways that may be affected by ENM exposure include the jugular and nodose ganglia, which receive feedback from pulmonary C-fibers and project to the cardiovascular center present in the medulla oblongata. Recently, pulmonary exposure to nano- $\mathrm{TiO}_{2}$ was shown to significantly increase heart rate and 
decrease diastolic function in rats treated with isoproterenol, a non-selective $\beta$ adrenoreceptor agonist, while elevating mean and diastolic pressure in response to norepinephrine [27]. Additionally, inhalation of MWCNT for 5 hours at a concentration of $5 \mathrm{mg} / \mathrm{m}^{3}$ altered the balance between sympathetic and parasympathetic inputs determined by changes in heart rate variability. An increase in sympathetic tone and decreased cardiac parasympathetic vagal tone may therefore be in part responsible for the cardiovascular effects observed post-exposure to ENM. ENM may also bypass the BBB by anterograde neuronal transport via the olfactory bulb [19]. Indeed, manganese oxide and nano- $\mathrm{TiO}_{2}$ have been shown to reach the striatum, frontal cortex, thalamus, hippocampus, cerebellum, and brain stem [15]. However, confirmation of autonomic dysfunction via this pathway requires further investigation.

\section{3) Developmental Origin of Health and Diseases}

This last section will address the effect of gestational ENM exposure on the vascular resistance of the maternal-fetal circulation $\backslash$. In study 1 we identified the pronounced sensitivity of the microcirculation to ENM exposure while in study 2 we proposed a novel potential immune link between the pulmonary route of exposure and the microvasculature. The aim of study 3 was to determine the effects of ENM exposure on the maternal-fetal circulation, particularly on the umbilical macrocirculation and on the placental microcirculation. Inhalation nano- $\mathrm{TiO}_{2}$ exposure decreased endothelium-dependent dilation in the umbilical artery while increasing ANGII sensitivity. Further, gestational ENM exposure decreased mean placental outflow pressure in response to $\mathrm{ACh}$, ANGII and $\mathrm{Ca}^{2+}$-free superfusate.

Overall, vascular resistance in the maternal-fetal circulation is increased by gestational ENM exposure. As mentioned above, the flow rate within a vessel is dependent upon the pressure 
differential within a vessel and the vascular resistance. Increases in resistance, due to an increase in vessel length, a decrease in vessel density or diameter will have the greatest impact on the ability of the placenta to adequately perfuse the developing fetus. Indeed, studies with AgNP (18$20 \mathrm{~nm}$ ) confirm the ability of ENM to translocate to the placenta and induce fetal resorption [25]. Nano- $\mathrm{TiO}_{2}$ has been shown to accumulate in the placenta and translocate to the developing fetus [24]. Gavage of $1 \mathrm{mg} / \mathrm{kg} /$ day nano- $\mathrm{TiO}_{2}$ also reduced the ratio of placenta/body weight by GD 13 in mice [41]. In the same study, impaired vascularization and fetal microvascular network development were noted.

The effects on placental structure and function have been attributed to an interplay between several mechanisms. AgNP have been shown to increase the proinflammatory cytokines IL-6, IL$1 \beta$, TNF $\alpha$ and MCP1 in the lungs of pregnant mice. Placental TNF $\alpha$, which plays a role in placental inflammation, and IL-1 $\beta$ secreted by infiltrating macrophages are also upregulated [22]. Inhibition of trophoblast proliferation and increased apoptosis by nuclear pyknosis through upregulation of caspase 3 and Bax and downregulation of Bcl-2 have been shown to occur in mice in response to nano- $\mathrm{TiO}_{2}$ exposure [56]. Nano- $\mathrm{TiO}_{2}$ also increases ROS levels in both the uterus and the placenta [56], causing a subsequent decrease in NO bioavailability, a mechanism which may in part account for the decreased sensitivity to ACh seen in study 3 .

Study 3 indicated an increase in sensitivity to ANGIl in umbilical vessels and placentas from nano$\mathrm{TiO}_{2}$ inhalation exposed animals. We investigated the influence of ENM exposure on the placental RAS response. AgNP and nano- $\mathrm{TiO}_{2}$ have been shown to cause an imbalance in the local brain RAS system via increased ROS levels and inflammation [54], resulting in increased levels of angiotensin converting enzyme and ANGII. Therefore, a similar effect may have occurred in the 
maternal-fetal vasculature and may explain the observations reported in study 3 . ENM exposure may lead to an imbalance in the ANGII receptor populations in the maternal-fetal circulation resulting in the increased expression and availability of AT1 receptor. ANGII acting via the AT1 receptor activates NADPH oxidase and causes impaired endothelial function and flow-mediated vasodilation, inflammation, atherosclerosis, vascular hypertrophy and inward remodeling during pathology [47]. These phenomena result in reduced maternal-fetal blood flow and may have significant effects on fetal development. Indeed, increases in ANGIl levels during gestation have been linked to decreased fetal size and preeclampsia [28].

Vasomotor tone is influenced by ANGII via activation of the AT1 receptors. ANGII binding of the AT1 receptor activates phospholipase $\mathrm{C}$, leading to the formation of the secondary messengers inositol-1-4-5-trisphosphate and diacylglycerol, which subsequently results in an increase in intracellular calcium levels and phosphorylation of myosin light chain and stimulation of sustained contraction [20]. Furthermore, ANGII may affect VSM contraction via the RhoA/Rho kinase pathway, leading to an increased sensitivity to intracellular concentrations of free calcium due to the inhibition of myosin light chain phosphatase [21]. Additionally, ANGll also increases the formation of NOX and arachidonic acid-derived ROS, thus stimulating the contraction of VSM [45]. ANGII has been shown to primarily target VSM but may also regulate endothelial transient receptor channels by influencing calcium-activated potassium which are involved in vascular contraction and relaxation [9]. Therefore, the observed effects of gestational nano- $\mathrm{TiO}_{2}$ exposure on maternal-fetal vascular resistance may involve an increase in sensitivity to ANGII as a result of an increase in AT1 receptor population which may lead to an increase in vascular tone. 


\section{4) Conclusions and Future Directions}

It is clear that the systemic and microvascular effects associated with pulmonary nano- $\mathrm{TiO}_{2}$ are extensive and involve several mechanisms, some of which have yet to be uncovered. Therefore, it was the objective of this dissertation work to propose ILC2 as potential innate immune effector cells associated with pulmonary ENM exposure and identify an understudied yet important target of ENM exposure (the placenta). There is extensive evidence indicating that nano- $\mathrm{TiO}_{2}$ impairs cardiovascular function in healthy male animal models but its effects on susceptible populations such as pregnant women and developing fetuses are unknown. The differential effects observed in pregnant and virgin animal models and the significant impact on fetal development, mandate more thorough investigations of the reproductive and developmental consequences of ENM.

Defining the physico-chemical properties (size, shape, surface charge, functionalization etc.) and the critical window for gestational exposure will also be important for the determination of the effects of ENM on fetal health. Unfortunately, studies 2 and 3 assessed the effect of a single dose and a single type of ENM. All of the experiments in study 3 were conducted on GD 20, while the exposures were initiated on GD 11, well after implantation. Future studies should focus on identifying the impact of different ENMs at different times prior to and during gestation and clarifying the link between physico-chemical properties and reproductive toxicity.

Research on the reproductive effects of ENM in general is still in its infancy and many intriguing questions remain unanswered. The studies in this dissertation work will be a stepping-stone for future work aimed at clarifying the acute inflammatory mechanisms and the role played by the placenta in ENM exposure. 


\section{Reference List:}

[1] Abukabda AB, Stapleton PA, Nurkiewicz TR. Metal Nanomaterial Toxicity Variations Within the Vascular System. Curr Environ Health Rep. 2016 Dec;3(4):379-391.

[2] Artis, D. and H. Spits, The biology of innate lymphoid cells. Nature, 2015. 517(7534): p. 293-301.

[3] Baenziger NL, Brodie GN, Majerus PW. A thrombin-sensitive protein of human platelet membranes. Proc Natl Acad Sci U S A. 1971. doi:10.1073/pnas.68.1.240

[4] Baisch, B.L., et al., Equivalent titanium dioxide nanoparticle deposition by intratracheal instillation and whole-body inhalation: the effect of dose rate on acute respiratory tract inflammation. Part Fibre Toxicol, 2014. 11: p. 5.

[5] Beamer, C.A., et al., IL-33 mediates multi-walled carbon nanotube (MWCNT)-induced airway hyper-reactivity via the mobilization of innate helper cells in the lung. Nanotoxicology, 2013. 7(6): p. 1070-81.

[6] Berne RM, Koeppen.B.M., Stanton BA. Berne \& Levy Physiology, Philadelphia, Mosby/Elsevier, 2010.

[7] Bonner JC, Silva RM, Taylor AJ, et al. Interlaboratory evaluation of rodent pulmonary responses to engineered nanomaterials: the NIEHS Nano GO Consortium. Environ Health Perspect 2013; $121676-82$

[8] Cassee FR, van Balen EC, Singh C, et al. Exposure, health and ecological effects review of engineered nanoscale cerium and cerium oxide associated with its use as a fuel additive. Crit Rev.Toxicol. 2011;41(3)213-229.

[9] Cat AND, Touyz RM. Cell signaling of angiotensin II on vascular tone: Novel mechanisms. Curr Hypertens Rep. 2011. doi:10.1007/s11906-011-0187-x 
[10] Champion JA, Walker A, Mitragotri S. Role of particle size in phagocytosis of polymeric microspheres. Pharm.Res. 2008;25(8)1815-1821.

[11] Courtois A, Andujar P, Ladeiro $\mathrm{Y}$, et al. Impairment of NO-dependent relaxation in intralobar pulmonary arteries: comparison of urban particulate matter and manufactured nanoparticles. Environ Health Perspect 2008; 116 1294-9

[12] Delmaschio A, Albors M, Evangelista V, et al. Measurement of Ionized Cytoplasmic Calcium Mobilization with the Photoprotein Aequorin in Human Polymorphonuclear Leukocytes Activated by Platelet Activating Factor (Paf). J Lipid Mediat. 1989.

[13] Dong M, Yan BP, Liao JK, Lam YY, Yip GWK, Yu CM. Rho-kinase inhibition: A novel therapeutic target for the treatment of cardiovascular diseases. Drug Discov Today. 2010. doi:10.1016/j.drudis.2010.06.011

[14] Eiserich JP, Baldus S, Brennan ML, et al. Myeloperoxidase, a leukocyte-derived vascular NO oxidase. Science (80- ). 2002. doi:10.1126/science.1106830

[15] Elder A, Gelein R, Silva V, et al. Translocation of inhaled ultrafine manganese oxide particles to the central nervous system. Environ Health Perspect. 2006;114(8):1172-1178. doi:10.1289/ehp.9030

[16] Forlow SB, Foley PL, Ley K. Severely reduced neutrophil adhesion and impaired host defense against fecal and commensal bacteria in CD18-/-P-selectin-/- double null mice. FASEB J 2002; 16 1488-96

[17] Fukata M, Kaibuchi K. Rho-family GTPases in cadherin-mediated cell-cell adhesion. Nat Rev Mol Cell Biol. 2001. doi:10.1038/35103068 
[18] G. C, N. C-M, E.E. K, et al. Thrombospondin-1 stimulates macrophage reactive oxygen species generation via CD47: A novel signaling event in atherosclerosis. Free Radic Biol Med. 2013.

[19] Grabrucker AM, Ruozi B, Belletti D, et al. Nanoparticle transport across the blood brain barrier. Tissue Barriers. 2016. doi:10.1080/21688370.2016.1153568

[20] Griendling KK, Ushio-Fukai M, Lassegue B, Alexander RW. Angiotensin II Signaling in Vascular Smooth Muscle: New Concepts. Hypertension. 1997. doi:10.1161/01.HYP.29.1.366

[21] Guilluy C, Brégeon J, Toumaniantz G, et al. The Rho exchange factor Arhgef1 mediates the effects of angiotensin II on vascular tone and blood pressure. Nat Med. 2010. doi:10.1038/nm.2079

[22] Guo H, Zhang J, Boudreau M, et al. Intravenous administration of silver nanoparticles causes organ toxicity through intracellular ROS-related loss of inter-endothelial junction. Part Fibre.Toxicol. 2016;1321.

[23] Hirano S, Fujitani Y, Furuyama A, et al. Uptake and cytotoxic effects of multi-walled carbon nanotubes in human bronchial epithelial cells. Toxicol.Appl.Pharmacol. 2010;249(1)8-15.

[24] Hougaard KS, Jackson P, Jensen KA, et al. Effects of prenatal exposure to surfacecoated nanosized titanium dioxide (UV-Titan). A study in mice. Part Fibre.Toxicol. 2010;716.

[25] Huang, J.P., et al., Nanoparticles can cross mouse placenta and induce trophoblast apoptosis. Placenta, 2015. 36(12): p. 1433-41.

[26] lii CAP, Verrier RL, Lovett EG, et al. Heart rate variability associated with particulate air pollution. :11-15. 
[27] Kan H, Wu Z, Lin Y-C, et al. The role of nodose ganglia in the regulation of cardiovascular function following pulmonary exposure to ultrafine titanium dioxide. Nanotoxicology. 2013:1-8. doi:10.3109/17435390.2013.796536

[28] Krebs, C., et al., Intrauterine growth restriction with absent end-diastolic flow velocity in the umbilical artery is associated with maldevelopment of the placental terminal villous tree. Am J Obstet Gynecol, 1996. 175(6): p. 1534-42.

[29] Learmont, J.G. and L. Poston, Nitric oxide is involved in flow-induced dilation of isolated human small fetoplacental arteries. Am J Obstet Gynecol, 1996. 174(2): p. 583-8.

[30] LeBlanc AJ, Moseley AM, Chen BT, et al. Nanoparticle inhalation impairs coronary microvascular reactivity via a local reactive oxygen species-dependent mechanism. Cardiovasc.Toxicol. 2010;10(1)27-36.

[31] Legramante JM, Valentini F, Magrini A, et al. Cardiac autonomic regulation after lung exposure to carbon nanotubes. Hum Exp Toxicol. 2009. doi:10.1177/0960327109105150

[32] Liu, X., et al., Anti-IL-33 antibody treatment inhibits airway inflammation in a murine model of allergic asthma. Biochem Biophys Res Commun, 2009. 386(1): p. 181-5.

[33] Mandler WK, Nurkiewicz TR, Porter DW, Olfert IM. Thrombospondin-1 mediates multiwalled carbon nanotube induced impairment of arteriolar dilation. Nanotoxicology. 2017. doi:10.1080/17435390.2016.1277275

[34] Mercer RR, Scabilloni JF, Hubbs AF, et al. Extrapulmonary transport of MWCNT following inhalation exposure. Part Fibre Toxicol 2013; 1038

[35] Michaudel, C., et al., Inflammasome, IL-1 and inflammation in ozone-induced lung injury. Am J Clin Exp Immunol, 2016. 5(1): p. 33-40. 
[36] Minarchick, V.C., et al., Cerium Dioxide Nanoparticle Exposure Improves Microvascular Dysfunction and Reduces Oxidative Stress in Spontaneously Hypertensive Rats. Front Physiol, 2015. 6: p. 339.

[37] Nemmar A, Delaunois A, Nemery B, et al. Inflammatory effect of intratracheal instillation of ultrafine particles in the rabbit: Role of C-fiber and mast cells. Toxicol Appl Pharmacol. 1999. doi:10.1006/taap.1999.8762

[38] Nurkiewicz TR, Porter DW, Barger M, et al. Systemic microvascular dysfunction and inflammation after pulmonary particulate matter exposure. Environ Health Perspect. 2006. doi:10.1289/ehp.8413

[39] Nurkiewicz TR, Porter DW, Hubbs AF, et al. Pulmonary nanoparticle exposure disrupts systemic microvascular nitric oxide signaling. Toxicol.Sci. 2009;110(1)191-203.

[40] Oberdorster G, Maynard A, Donaldson K, et al. Principles for characterizing the potential human health effects from exposure to nanomaterials: elements of a screening strategy. Part Fibre.Toxicol. 2005;28.

[41] Parivar, K., et al., The Effect of Nano-Titanium Dioxide on Limb Bud Development of NMRI Mouse Embryo In Vivo. Cell J, 2015. 17(2): p. 296-303.

[42] Pries AR, Secomb TW, Gaehtgens P. Biophysical aspects of blood flow in the microvasculature. Cardiovasc.Res. 1996;32(4)654-667.

[43] Pries AR, Secomb TW. Rheology of the microcirculation. Clin.Hemorheol.Microcirc. 2003;29(3-4)143-148.

[44] Rajagopalan S, Brook RD. The Indoor-Outdoor Air-Pollution Continuum and the Burden of Cardiovascular Disease: An Opportunity For Improving Global Health. Glob.Heart 2012;7(3)207-213. 
[45] Rajagopalan S, Kurz S, Münzel T, et al. Angiotensin II-mediated hypertension in the rat increases vascular superoxide production via membrane NADH/NADPH oxidase activation: Contribution to alterations of vasomotor tone. J Clin Invest. 1996. doi:10.1172/JCl118623

[46] Schwartzkopff B, Mundhenke M, Strauer BE. Alterations of the architecture of subendocardial arterioles in patients with hypertrophic cardiomyopathy and impaired coronary vasodilator reserve: a possible cause for myocardial ischemia. J Am Coll Cardiol 1998; 31 108996.

[47] Shah, D.M., Role of the renin-angiotensin system in the pathogenesis of preeclampsia. Am J Physiol Renal Physiol, 2005. 288(4): p. F614-25.

[48] Spits, H., et al., Innate lymphoid cells--a proposal for uniform nomenclature. Nat Rev Immunol, 2013. 13(2): p. 145-9.

[49] Stapleton PA, Abukabda AB, Hardy SL, Nurkiewicz TR. Xenobiotic pulmonary exposure and systemic cardiovascular response via neurological links. Am J Physiol Circ Physiol. 2015. doi:10.1152/ajpheart.00546.2015

[50] Stapleton PA, Nurkiewicz TR. Vascular distribution of nanomaterials. Wiley.Interdiscip.Rev.Nanomed.Nanobiotechnol. 2014;6(4)338-348.

[51] Stapleton, P.A. and T.R. Nurkiewicz, Maternal nanomaterial exposure: a double threat to maternal uterine health and fetal development? Nanomedicine (Lond), 2014. 9(7): p. 929-31.

[52] Thompson LC VA. Vascular Tissue Contractility Changes Following Late Gestational Exposure to Multi-Walled Carbon Nanotubes or their Dispersing Vehicle in Sprague Dawley Rats. J Nanomed Nanotechnol. 2014. doi:10.4172/2157-7439.1000201 
[53] Trickler WJ, Lantz SM, Murdock RC, et al. Silver nanoparticle induced blood-brain barrier inflammation and increased permeability in primary rat brain microvessel endothelial cells. Toxicol.Sci. 2010;118(1)160-170.

[54] Wang J, Byrne JD, Napier ME, et al. More effective nanomedicines through particle design. Small 2011;7(14)1919-1931.

[55] Yamashita, K. and Y. Yoshioka, [Safety assessment of nanomaterials in reproductive developmental field]. Yakugaku Zasshi, 2012. 132(3): p. 331-5.

[56] Yang, Q., et al., Group 2 innate lymphoid cells mediate ozone-induced airway inflammation and hyperresponsiveness in mice. J Allergy Clin Immunol, 2016. 137(2): p. 571-8.

[57] Zoltowska, A.M., et al., The interleukin-33 receptor ST2 is important for the development of peripheral airway hyperresponsiveness and inflammation in a house dust mite mouse model of asthma. Clin Exp Allergy, 2016. 46(3): p. 479-489 


\section{Appendices}

\section{Appendix A:}

Supplementary Figure 1. IL-33 sequence homology. Multiple sequence alignment of the protein sequence of IL-33 in mice (query) and rats (subject) showing the significant interspecies homology of murine IL-33.

\section{Appendix B:}

Supplementary Figure 1. Maternal nano- $\mathrm{TiO}_{2}$ inhalation exposure impairs endotheliumdependent placental hemodynamics. Regression analysis showing that maternal nano- $\mathrm{TiO}_{2}$ inhalation exposure alters placental hemodynamics and decreases outflow (venous) pressure in placentas in normal superfusate $(A)$ and placentas treated with $A C h(B)(n=8) .{ }^{*}, P \leq 0.05$ shamcontrol group vs. nano-TiO2 exposed group.

Supplementary Figure 2. Maternal nano- $\mathrm{TiO}_{2}$ inhalation exposure does not affect endothelium-independent placental hemodynamics but increases ANGll sensitivity. Regression analysis showing that maternal nano- $\mathrm{TiO}_{2}$ inhalation exposure did not affect endothelial-independent placental hemodynamics (A) but increased ANGIl sensitivity (B) $(n=8)$. ${ }^{*}, \mathrm{P} \leq 0.05$ sham-control group vs. nano-TiO2 exposed group.

Supplementary Figure 3. Maternal nano- $\mathrm{TiO}_{2}$ inhalation impairs calcium-free placental hemodynamics. Regression analysis showing that maternal nano- $\mathrm{TiO}_{2}$ inhalation exposure alters placental hemodynamics and decreases outflow (venous) pressure in placentas in calciumfree superfusate $(n=8)$. ${ }^{*}, P \leq 0.05$ sham-control group vs. nano- $\mathrm{TiO}_{2}$ exposed group. 


\section{Appendix A}

\section{Supplementary Figure 1:}

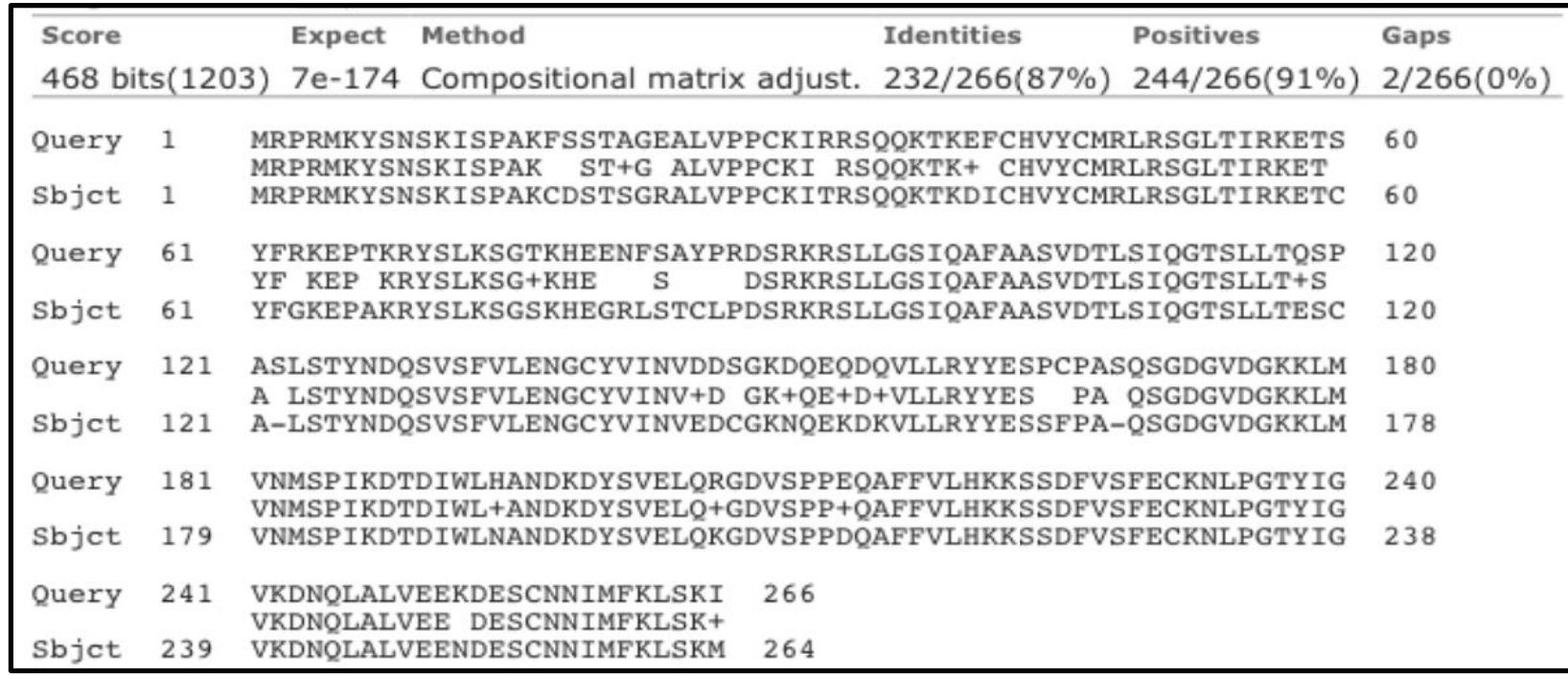

\begin{tabular}{|l|l|l|l|l|l|}
\hline & $\begin{array}{l}\text { Max } \\
\text { Query }\end{array}$ & $\begin{array}{l}\text { Total } \\
\text { Query }\end{array}$ & $\begin{array}{l}\text { Query } \\
\text { Cover }\end{array}$ & E value & Identity \\
\hline Interleukin-33 & 466 & 466 & $100 \%$ & $7 \mathrm{e}-174$ & $87 \%$ \\
\hline
\end{tabular}




\section{Appendix B}

\section{Supplementary Figure 1:}
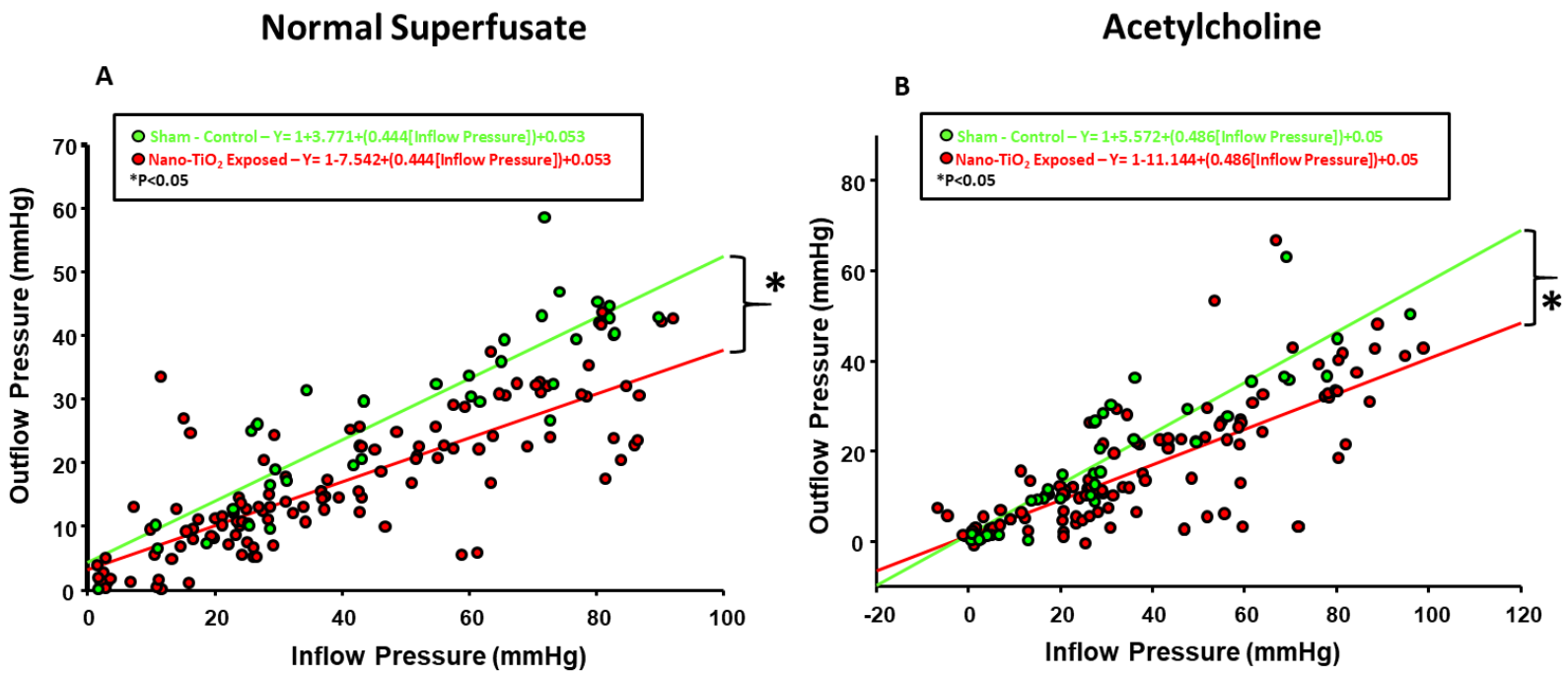


\section{Supplementary Figure 2:}
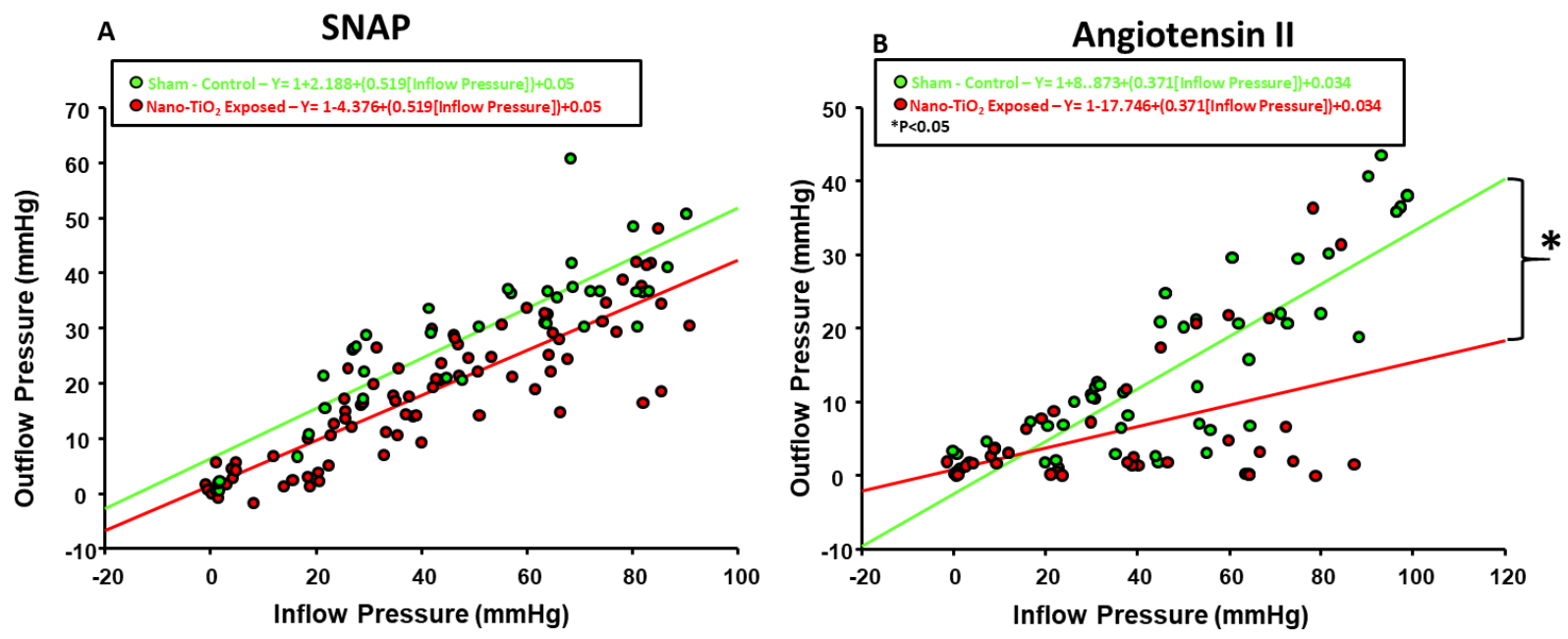
Supplementary Figure 4:

\section{Calcium-Free Superfusate}

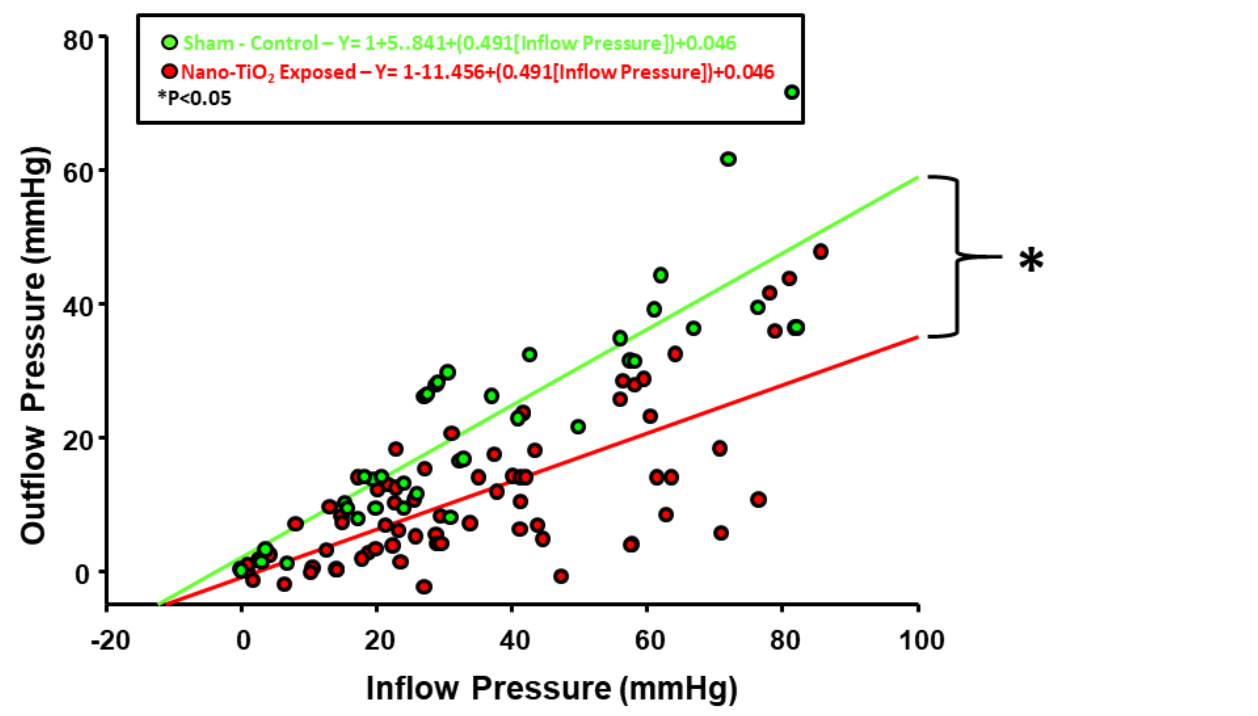




\title{
VII. Curriculum Vitae
}

\section{Alaeddin Bashir Abukabda}

\author{
Physiology and Pharmacology Department \\ Robert C. Byrd Health Sciences Center \\ 64 Medical Center Drive West Virginia University \\ Morgantown, WV 26506-9105 \\ Email: ababukabda@mix.wvu.edu
}

\section{Education}

Al Fateh University (2002-2006)

Department: Dentistry

Major: Dentistry

Degree Conferred: D.M.D

Clarion University of Pennsylvania (2008-2011)

School: Arts \& Sciences Department: Biology

Concentration: Molecular Biology Degree

Conferred: M.Sc.

West Virginia University (2014-2018)

School: Medicine

Division: Cellular and Integrative Physiology

Degree: $\mathrm{PhD}$

West Virginia University (2016-2018)

School: Public Health

Division: Epidemiology and Biostatistics

Degree: Certification in Biostatistics 


\section{History}

2009-2011 - Clarion University of PA - Laboratory Assistant for Anatomy and Physiology Laboratory Assistant for Introduction to Biology

2009-2011 - Clarion University of PA - Graduate Assistant,

Molecular Biology, Master's Candidate

Department of Biology Advisor: Douglas Smith

2009-2011 - Clarion Research Group - Researcher

2014-2018 - West Virginia University - Graduate Research Assistant, Department of Physiology and Pharmacology Advisor: Timothy R. Nurkiewicz, Ph. D

\section{Previous Teaching Experience}

Biology I and II lab and lecture - 2009-2011

Practice Based Learning Class - 2018

Cell Methods - 2016-2017

\section{Awards/Achievements}

- Van Liere Research Day Award - 2018

- Maryanne Stock Research Award - 2018

- Cardiovascular Toxicology Specialty Section Student Award - 2018

- Reproductive Toxicology Specialty Section Student Award - 2018

- Julie Betschart Award - 2017

- American Public Health Association Award - 2017

- FASEB MARC Travel Award - 2017

- Society of Teratology Travel Award - 2017

- Society of Toxicology Travel Award - 2017

- International Nanotoxicology Congress Travel Award - 2016 
- Joint Regional American Association of Pharmaceutical Scientists (AAPS) 2015 and International Society for Pharmcoeconomics and Outcomes Research (ISPOR), Morgantown WV

- Clarion University Outstanding Graduate Student Award - 2011

- Clarion University Research Award - 2010

○ Dean's List 2008-2011

- NESA Ugrad - Fulbright Scholarship 2008

- Robert Moore Award for Academic Excellence 1998-2000

\section{Positions}

o Member, Center for Cardiovascular and Respiratory Sciences 2015-present

o Member, The Microcirculation Society 2015-present

o Member, Society of Toxicology 2015-present

o Member, Alleghany-Erie Regional Chapter SOT 2015-present

o Member, National Society for Leadership and Success 2009-2011

\section{Invited Symposia}

o WVU, Department of Physiology and Pharmacology, Morgantown,

WV. (2015): "Nano-Titanium Dioxide Differentially Impairs Segmental Vascular Reactivity and Endothelial Cell Function". o Research and Education in Nanotoxicology at West Virginia

Interdisciplinary Graduate Education and Research Traineeship and NanotechnologyEnvironmental Effects and Policy (REN@WVU- NEEP) Collaborative Symposium. (2015): "Exposure to Nano-titanium Dioxide Differentially Impacts Vascular Cholenergic Sensitivity."

o WVU, Department of Physiology and Pharmacology, Morgantown, WV. (2014): "Effects of Acute Exposure to Nanomaterials on Macrovascular Reactivity". 


\section{Publications}

\section{Peer Reviewed Manuscripts}

1. Abukabda, A.B., et al., Group II innate lymphoid cells and microvascular dysfunction from pulmonary titanium dioxide nanoparticle exposure. Part Fibre Toxicol, 2018. 15(1): p. 43.

2. Stapleton, P.A., et al., Estrous cycle-dependent modulation of in vivo microvascular dysfunction after nanomaterial inhalation. Reprod Toxicol, 2018. 78: p. 20-28.

3. Stapleton, P.A., et al., Maternal engineered nanomaterial inhalation during gestation alters the fetal transcriptome. Part Fibre Toxicol, 2018. 15(1): p. 3.

4. Nichols, C.E., et al., Reactive oxygen species damage drives cardiac and mitochondrial dysfunction following acute nano-titanium dioxide inhalation exposure. Nanotoxicology, 2018. 12(1): p. 32-48.

5. Stefaniak, A.B., et al., Inhalation exposure to three-dimensional printer emissions stimulates acute hypertension and microvascular dysfunction. Toxicol Appl Pharmacol, 2017. 335: p. 1-5.

6. Abukabda, A.B., et al., Heterogeneous Vascular Bed Responses to Pulmonary Titanium Dioxide Nanoparticle Exposure. Front Cardiovasc Med, 2017. 4: p. 33.

7. Hathaway, Q.A., et al., Maternal-engineered nanomaterial exposure disrupts progeny cardiac function and bioenergetics. Am J Physiol Heart Circ Physiol, 2017. 312(3): p. H446-H458. 8. Abukabda, A.B., P.A. Stapleton, and T.R. Nurkiewicz, Metal Nanomaterial Toxicity Variations Within the Vascular System. Curr Environ Health Rep, 2016. 3(4): p. 379-391.

9. Stapleton, P.A., et al., Xenobiotic pulmonary exposure and systemic cardiovascular response via neurological links. Am J Physiol Heart Circ Physiol, 2015. 309(10): p. H1609-20.

10. Davis, V.L., et al., Platelet-rich preparations to improve healing. Part II: platelet activation and enrichment, leukocyte inclusion, and other selection criteria. J Oral Implantol, 2014. 40(4): p. $511-21$. 
11. Davis, V.L., et al., Platelet-rich preparations to improve healing. Part I: workable options for every size practice. J Oral Implantol, 2014. 40(4): p. 500-10.

\section{Book Chapter}

1. Stapleton PA, Abukabda AB, Nurkiewicz TR. "Assessment of Vascular Reactivity" in: Cardiovascular Toxicology, of Cardiovascular Physiology 3rd edition (in preparation)

2. Stapleton PA, Abukabda AB, Nurkiewicz TR. "Principles of Toxicology" in: Modern Pharmacology, (in review)

3. "Complications of Oral Implantology" - Quintessence Publishing, 2011

\section{Abstracts}

1. Abukabda AB, Stapleton PA, McBride CR, Yi J, Nurkiewicz TR. Heterogeneity of the Vascular Response to Acute Pulmonary Nano-TiO2 Exposure. The Toxicologist (2017)

2. Nichols CE, Hollander JM, Engels K, Abukabda AB, Stapleton PA, Nurkiewicz TR. Maternal Nanomaterial Inhalation During Gestation Impairs Microvascular Reactivity and Alters the Fetal Genome. The Toxicologist (2016)

3. Abukabda AB, Stapleton PA, Nurkiewicz. Nano-Titanium Dioxide Exposure Differentially Affects Segmental Vascular Reactivity. The Toxicologist (2016)

4. Abukabda AB, Stapleton PA, Nurkiewicz. Exposure to Nano- titanium Dioxide Differentially Impacts Vascular Cholenergic Sensitivity. Research and Education in Nanotoxicology at West Virginia Interdisciplinary Graduate Education and Research Traineeship and NanotechnologyEnvironmental Effects and Policy (REN@WVU-NEEP) Collaborative Symposium. (2015) 
5. Simon SA, Stapleton PA, Abukabda AB, McBride CR, Nurkiewicz, TR. Engineered Nanomaterial Effects on the Cardiovascular Effects. West Virginia IDeA Network of Biomedical Research Excellence (WV- INBRE), Huntington, WV (2015)

6. Krupa RC, Stapleton PA, Abukabda AB, Minarchick VC, Nurkiewicz, TR. Impact of nanomaterial Exposure on the Reactivity of Small Arteries in the Mesentery. WVNano Research Experiences for Undergraduate, Summer Undergraduate Research Symposium, Morgantown, WV (2015)

7. Abukabda AB, Stapleton PA, Nurkiewicz, TR. Acute Exposure to Nano-Titanium Dioxide Differentially Impairs Endothelial Cellular Signaling. Joint Regional American Association of Pharmaceutical Scientists (AAPS) and International Society for Pharmcoeconomics and Outcomes Research (ISPOR), Morgantown WV (2015)

8. Abukabda AB, Stapleton PA, Nurkiewicz, TR. Differential Effects of Nano-Titanium Dioxide Exposure on Vascular Reactivity. Alleghany-Erie Society of Toxicology (2015)

9. Abukabda AB, Stapleton PA, Nurkiewicz, TR. Dose-Dependent Effect of Nano-Titanium Dioxide Exposure on Aortic Reactivity. West Virginia University, Van Liere Research Day (2015).

\section{Student Mentorship and Supervision}

o Julie Griffith - Fall 2018

o Krista Garner - Fall 2017

o Elizabeth Bowdridge - Fall 2017

o Wijdan Dabeek, Research Experience - Fall 2016 - 2017

o Spencer Duffey, Pharmacy Student, Research Internship - Fall 2014 - present 
o Andrew Nickerson, Graduate Research Rotation - Fall 2015

o Samantha Simon, WVU INBRE and Health Science Technology Academy (HSTA) Program, University High School, Morgantown WV - Summer 2015

o Rebekah Krupa, Research Experience for Undergraduates, WVU NanoSAFE - Summer 2015

\section{Professional Journal Activities}

Ad Hoc Reviewer

o American Journal of Physiology- Lung, Cellular and Molecular Physiology o Environmental Health Perspectives

o Cardiovascular Toxicology

o Chemosphere

o Inhalation Toxicology

o Journal of Nanomedicine and Nanotechnology

o Journal of Toxicology and Environmental Health o Journal of Visualized Experiments

o Life Sciences

o Medicine and Science in Sports and Exercise o Molecules

o Microcirculation o Nanomedicine

o Nanotoxicology

o Nigerian Medical Journal

o Particle and Fibre Toxicology

o PLOS ONE 
o Reproductive Toxicology

o Toxicology and Applied Pharmacology

o WIREs Nanomedicine and Nanobiotechnology o Toxicological Sciences

o Journal of Toxicology and Applied Pharmacology

\section{Memberships (Past and Current)}

o The Microcirculation Society

o The Center for Cardiovascular and Respiratory Sciences

o Society of Toxicology

- Cardiovascular Toxicology Specialty Section

- Nanotoxicology Specialty Section

- Immunotoxicology Specialty Section

- Inhalation Toxicology Specialty Section

- Toxicologists of African Origin

- Allegheny-Erie Regional Chapter

- The Teratology Society - Education Committee and Awards Committee

-American Public Health Association 


\section{Participation in Scientific Meetings}

o International Nanotoxicology Congress $(2016,2018)$

o Society of Toxicology National Meeting (2016-2018).

o Allegheny-Erie Regional Chapter of the Society of Toxicology Annual Meeting (2015-2018).

o The Teratology Society National Meeting (2017)

o American Public Health Association Annual Meeting (2017)

\section{Continuing Education Symposia/Webinar}

o Betschart Symposium, West Virginia University $(2016,2017)$

o Grant Writers' Seminar and Workshop (2015)

o Research and Education in Nanotoxicology at West Virginia Interdisciplinary Graduate Education and Research Traineeship and Nanotechnology-Environmental Effects and Policy (REN@WVU- NEEP) Collaborative Symposium. (2015)

o Joint Regional American Association of Pharmaceutical Scientists (AAPS) and International Society for Pharmcoeconomics and Outcomes Research (ISPOR), Morgantown WV $(2015,2018)$ o Van Liere Research Day, West Virginia University (2015-2018). 